Universidad Politécnica de Madrid

Escuela Técnica Superior de Ingenieros Agrónomos

Departamento de Biotecnología

\title{
Genomics of Specificity in the Symbiotic Interaction between Rhizobium leguminosarum and Legumes
}

Ph.D Thesis

Beatriz Jorrín Rubio

Licenciada en Biología

Madrid, 2016 




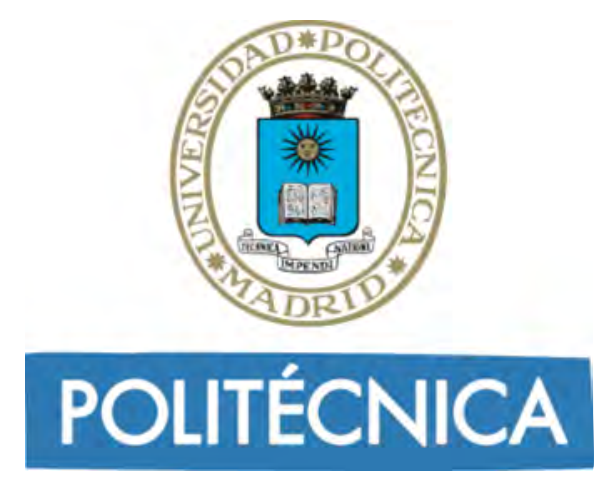

\title{
Universidad Politécnica de Madrid
}

Escuela Técnica Superior de Ingenieros Agrónomos

Genomics of Specificity in the Symbiotic Interaction between Rhizobium leguminosarum and Legumes

\author{
Ph.D Thesis \\ Beatriz Jorrín Rubio \\ Licenciada en Biología \\ 2016
}





\section{Universidad Politécnica de Madrid \\ Escuela Técnica Superior de Ingenieros Agrónomos Departamento de Biotecnología}

Ph.D Thesis:

Genomics of Specificity in the Symbiotic Interaction between Rhizobium leguminosarum and Legumes

Author:

Beatriz Jorrín Rubio

Licenciada en Biología

Director:

Juan Imperial Ródenas

Licenciado en Biología

Doctor en Biología

Madrid, 2016 

A mis padres

A Sofía

A mis hermanos 

"There's the story, then there's the real story, then there's the story of how the story came to be told. Then there's what you leave out of the story. Which is part of the story too."

Maddaddam Margaret Atwood 



\section{RECONOCIMIENTOS}

Esta Tesis se ha desarrollado en el laboratorio de Genómica y Biotecnología de Bacterias Diazotróficas Asociadas con Plantas del Centro de Biotecnología y Genómica de Plantas (UPM-INIA). Para el desarrollo de esta Tesis he contado con una beca UPM homologada financiada por el proyecto MICROGEN: Genómica Comparada Microbiana (Programa Consolider), que ha sido también el proyecto financiador del trabajo experimental.

Quisiera reconocer la labor de aquellas personas que han contribuido al desarrollo y consecución de esta Tesis.

Dr. Juan Imperial, por la dirección y supervisión de esta Tesis. Por ser un gran mentor y por enseñarme todo lo que sé.

Dr. Manuel González Guerrero, por enseñarme los entresijos de la Ciencia y del trabajo en el laboratorio.

Dra. Gisèle Laguerre, por su participación en el inicio de este proyecto y por facilitarnos el suelo P1 de Dijon.

Dr. Paulo Arruda, por la formación que obtuve durante mi estancia en el laboratorio de Estudio de la Regulación y de la Expresión génica de la Universidad Estatal de Campinas.

Dra. Belén Brito, por su gran ayuda en la primera fase de este proyecto y por recomendarme, sin ella nunca hubiera empezado esta Tesis.

Rosabel Prieto, por su inestimable ayuda para la obtención de los Pool-Seqs y por enseñarme muchas de las técnicas que he necesitado durante la Tesis.

Santiago de la Peña, por su valiosa ayuda en el análisis bioinformático de los genomas individuales.

Dra. Julia Quintana, por enseñarme los conceptos de genética de poblaciones y por las largas discusiones científicas que tanto me han aportado.

Dra. Viviana Escudero, por enseñarme a cuidar las plantas y valorar las cosas realmente importantes.

Dra. Carmen Sánchez, por enseñarme las técnicas básicas de microbiología y genética molecular durante mi periodo como estudiante de máster.

Dr. David Durán, por enseñarme los conceptos básicos de filogenia. 



\section{AGRADECIMIENTOS}

Escribir los agradecimientos es la parte más difícil de una tesis, no porque sea complicado, si no porque es la única parte que se lee todo el mundo y supone una gran presión añadida. Siempre me imaginaba escribir esta parte como algo extremadamente emotivo, pero después de escribir más de 200 páginas me he quedado sin palabras.

La ciencia siempre fue lo que quise hacer, pero nunca pensé que pudiera hacerlo, pero... here I am! Ha sido un camino largo y duro, pero no lo cambiaría por nada, me he convertido en lo que quería y he conocido a los mejores. Desde que tengo 13 años sabía que quería estudiar esto, gracias a la inspiración de mi profesora de biología Marisa Alonso. Cuando llegué a la carrera, las cosas no fueron como esperaba, pero aún así descubrí mi pasión por la micro, la genética y las plantas... Y sobre todo conocí a grandes personas. Desde luego la mejor decisión que tomé fue hacer el máster, este fue el punto de inflexión, descubrí que era hacer ciencia... y eso me enganchó. Quiero agradecer a Pepe Palacios por acogerme en su laboratorio durante este periodo, ya que sin duda, mi paso por allí marcó mi vuelta. Por ello también quiero agradecer a todos los Rizobios y Levaduros, y en especial a Carmen Sánchez, por ser mi supervisora en los inicios y enseñarme muchas de las técnicas básicas de micro y genética molecular que he aprendido. No me puedo olvidar de dar las gracias a Tomás Ruíz Argüeso, siempre ha estado ahí cuando lo he necesitado, incluso acompañándome a fumar un cigarro. Indudablemente gracias a Belén Brito, por recomendarme y por ver lo mejor en mí. Y a los que hablaron con Belén, igracias!

Empezar la Tesis ha sido lo mejor que me ha pasado en mi carrera profesional. Sin duda tengo el mejor director de Tesis posible, Juan. ¡Muchas gracias! Gracias por el conocimiento, la experiencia y la libertad para desarrollarme como científica, por ser un gran mentor y mejor persona, ¡GRACIAS! Yo soy yo y mi circunstancia, y en mi circunstancia científica está Manuel González Guerrero, gracias por dejarme acompañarte en tu punto de inflexión, por enseñarme el alma de la ciencia, por no dejarme nunca bajar los brazos y por ser mi amigo. Gracias a Rosabel, sin su ayuda, no solo como técnico, si no también como persona, este proyecto hubiera sido muchísimo más difícil. Gracias a todos mis compañeros: Rosario, Marta, Amalia, Javier, Nuria, Manolo, Vivi, Isidro, Patricia y Rosabel; siempre habéis hecho del laboratorio un lugar fantástico donde trabajar. Gracias a todos los estudiantes que han estado a mi cargo: Adriana, Amalia, Miguel y Merce. Es más difícil enseñar que aprender, pero con vosotros como conejillos de indias ha sido más fácil. Gracias a 
todas las personas que han pasado por el laboratorio, y en especial a Benja, iqué grande eres!

EI CBGP ha sido mi casa durante estos cinco años, sin el personal de SEL (María y Carmen), del Invernadero (Mar y Chendo), de administración (Sonia, Jesús y Alejandro) de informática (Gonzalo) o de conserjería (Antonio), nada hubiera sido igual, muchas gracias a todos. A demás he conocido a grandes personas que se han convertido en buenos amigos: gracias a Lucía, Sara, Carlos, Balta, Manuel Guillermo, Marce y Rafa. Gracias a los Diazotroopers de Luis Rubio, por los grandes momentos en los congresos. Gracias a Rocío, Alonso, Aurora, Mónica y a mis colegas de cigarro.

Gracias a Julia, por creer en mi, por entenderme, por grandes momentos que se han convertido en recuerdos inolvidables y por todo lo que nos queda por compartir, ¡Gracias!. Gracias a Vivi, por ser como eres, tan suave... Por tu sinceridad, por tus jarros de realidad y por tu cariño. Sin vosotras también tendría una tesis, pero hubiera sido mucho más aburrido y mucho menos enriquecedor.

A mis amigas con las que empecé la carrera, Lore, Rocío, Gonzalo (una más) y Alba. Because we're worth it! Ya son más de trece años... ipor muchos más!

A mis cookies, Sandra y Elena. Gracias por confiar en mi. Por las tardes de bolos, las habitaciones rojas, los paseos en bici hasta el infinito y por el Pad Thai.

A mis amiguinxis con las que acabé la carrera, Ana, Elisa, Yvonne, Bea, Gloria, Raquel, Yolanda, Lidia y Cristina. Como dijo Miss Tegucigalpa del mismo modo en sentido contrario: "no cambiéis, ¡Grasias!"

A mis festivaleras, Paula, Patri y Gema. Por el xamón, los furaños, la buena música, los gintonics, los jägers y la playa; ya sabéis, venimos del mismo lugar...

A mis amigas del colegio, Ana, Mónica y Laura. Porque aunque pasen los años y evolucionemos, parece que aún seguimos teniendo quince años jugando a "Celeste" en tecnología.

Y por último, pero no por ello menos importante, gracias a mi familia. Gracias a mis padres, han sido, son y siguen siendo el mayor apoyo que he tenido nunca. Sin ellos no estaría hoy aquí y no sería quien soy. Gracias Mamá, Gracias Papá. A mi hermano Gonzalo, no se puede tener un hermano mejor y no me has podido hacer mejor regalo. Gracias a "my sister-in-law" Ximena, por regalarme a Sofía y por las conversaciones en la cocina. A mi sobri, por alegrarme cada día desde que existes, espero que siempre puedas estar orgullosa de tu tía. A toda mi familia, que siempre se ha preocupado por mi Tesis; a mi abuelo que siempre me dice "esto que haces tú sale mucho en los periódicos, es importante". A mis tíos, mis padrinos, mi padrina, mis primos, mis enanos, los que llegan y los que se han ido. ¡Gracias!

A todos ¡mil gracias! 


\section{ABSTRACT}

For centuries legumes have been used in crop rotations to incorporate nitrogen into agricultural systems, thus avoiding the need for fertilization. This is possible because of their symbiotic interaction with specific diazotrophic soil bacteria, collectively known as rhizobia, that infect legume roots, form root nodules and fix atmospheric nitrogen into ammonia using the nitrogenase enzyme. The establishment and maintenance of this symbiotic partnership results from a molecular conversation that guarantees specificity and guides the co-development of both organisms during root nodule formation. Rhizobium leguminosarum bv. viciae is a member of the $\alpha$-Proteobacteria that can establish effective symbioses with members of the Fabeae legume tribe (Pisum, Lathryrus, Lens and Vicia). Previous studies have suggested that, although all $R$. leguminosarum bv. viciae isolates can effectively nodulate all Fabeae, different Fabeae select specific genotypes of rhizobia from those available in soil.

The aim of this Thesis was to characterize the genomic, genetic and molecular bases of the selection of specific $R$. leguminosarum bv. viciae genotypes by different host legumes (Pisum sativum, Lens culinaris, Vicia faba and V. sativa) from a wellcharacterized agricultural soil.

We established a population genomics methodology based on pooled DNA samples (Pool-Seq) from $R$. leguminosarum isolates obtained from different sources: legume plant hosts used as rhizobial traps ( $P$. sativum, L. culinaris, V. sativa and $V$. faba), as well as the isolation of $R$. leguminosarum directly from soil. This approach allowed us to confirm the hypothesis that different plant hosts select specific subpopulations of rhizobia from the available population present in the soil.

We also set out to characterize the indigenous $R$. leguminosarum soil population avoiding plant selection, in order to compare it with previously characterized hostselected subpopulations from this soil. As a side result, we uncovered a large number of previously uncharacterized, non-symbiotic rhizobia that contribute to the population pangenome. Host preference for specific genotypes was especially relevant in the case of pea plants. They selected a $R$. leguminosarum population significantly different from that present in the soil. Quite on the contrary, lentil and fava bean plants did not show a significant genotype selection, and their nodule rhizobial populations reflected that present in soil (after one life cycle). Vetch plants revealed a certain genotypic preference, but not as substantial or as important as that from pea plants.

Given that plants can differentially select rhizobial genotypes and that viable rhizobia of those genotypes are released into soil after nodule senescence, we hypothesized that, in natural conditions after numerous cycles of selection-release, 
most nodules would be occupied by the preferred genotype/s in each plant. We experimentally tested this hypothesis in a mesocosm experiment aimed at mimicking these field conditions. We were able to demonstrate that the different plant hosts employed ( $P$. sativum, L. culinaris, $V$. faba and $V$. sativa) selected different genotypes from those available in the P1 soil, and that the basis of selection was different for different plants. Pea and fava bean plants strongly selected for specific genotypes, but in different ways. Pea nodules were colonized by strains endowed with a large set of genes probably implicated in rhizospheric fitness, irrespective of the symbiotic genotype they harboured. This suggestion should be confirmed by in situ transcriptomic studies. Fava bean plants restricted their selection to a specific symbiotic genotype that was not always localized in the same symbiotic plasmid, or within the same chromosomal background. No hard conclusions could be obtained for vetch, although we suggest that this plant might behave dually, either as a selective host if a given genotype results in a rhizospheric advantage (such as in vetch $_{B}$ subpopulation), or as a non-selective host, with its nodules reflecting the genotypic

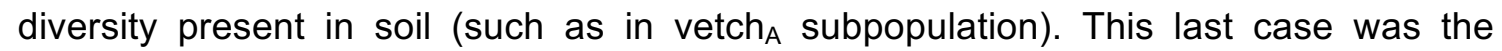
situation found for lentils; none of the three subpopulations isolated from lentil nodules (initial lentil, lentil $\mathrm{A}_{\mathrm{A}}$ and lenti $_{\mathrm{B}}$ ) differed significantly, either among themselves, regardless of the number of plant selection cycles, or with respect to the initial soil population. 


\section{RESUMEN}

Durante siglos las leguminosas se han usado en rotación de cultivos para incorporar nitrógeno a los sistemas agrícolas y así evitar el uso de fertilizantes nitrogenados. Esto ocurre gracias a su interacción simbiótica con los rizobios, bacterias diazotróficas del suelo que infectan las raíces de las leguminosas formando nódulos y fijando nitrógeno atmosférico a amonio gracias a la enzima nitrogenasa. El establecimiento y mantenimiento de la simbiosis conlleva un diálogo molecular que garantiza la especificidad y guía el co-desarrollo de ambos organismos durante la formación del nódulo. Rhizobium leguminosarum bv. viciae es un miembro del grupo de las $\alpha$-Proteobacterias que establece simbiosis con miembros la tribu Fabeae (Pisum, Lathryrus, Lens and Vicia). Estudios previos sugieren que, pese a que todos los aislamientos de $R$. leguminosarum bv. viciae nodulan eficientemente a todas las Fabeae, diferentes plantas seleccionan genotipos rizobianos específicos de entre los presentes en el suelo.

El objetivo de esta Tesis ha sido la caracterización genómica, genética y molecular de la selección de genotipos específicos de $R$. leguminosarum bv. viciae por diferentes huéspedes (Pisum sativum, Lens culinaris, Vicia faba and V. sativa) a partir de un suelo agrícola bien caracterizado.

Se ha desarrollado y utilizado una aproximación basada en genómica de poblaciones a partir de muestras conjuntas de ADN (Pool-Seq) de aislamientos de $R$. leguminosarum obtenidos a partir de leguminosas usadas como plantas trampa $(P$. sativum, L. culinaris, V. sativa and V. faba), así como R. leguminosarum aislados directamente del suelo. Esta metodología permitió confirmar la hipótesis de que diferentes plantas seleccionan subpoblaciones específicas de entre aquellos rizobios presentes en el suelo.

Se ha caracterizado la población nativa de $R$. leguminosarum del suelo, evitando el paso por la planta, para comparar con las poblaciones previamente caracterizadas aisladas de los nódulos de las diferentes plantas. Adicionalmente, esto nos permitió descubrir la existencia de un gran número de rizobios no simbióticos que contribuyen al pangenoma de la población. La preferencia por genotipos rizobianos específicos fue especialmente relevante en el caso del guisante, que seleccionó una población de $R$. leguminosarum significativamente diferente de la del suelo. Por el contrario, las lentejas y las habas no seleccionaron genotipos específicos significativamente diferentes de los del suelo tras un ciclo de selección. Las vezas mostraron cierta preferencia por algunos genotipos, pero no tan importante como en guisantes. 
Dado que los rizobios viables seleccionados por la planta son liberados al suelo tras la senescencia del nódulo, se puede hipotetizar que, en condiciones naturales, tras numerosos ciclos de selección-liberación, la mayoría de los nódulos deberían estar ocupados por el/los genotipo/s preferidos por cada planta. Esta hipótesis ha sido probada experimentalmente en un experimento de mesocosmos que trata de mimetizar las condiciones de campo. Hemos podido demostrar que las diferentes plantas empleadas ( $P$. sativum, $L$. culinaris, V. faba and $V$. sativa) seleccionan diferentes genotipos de entre los disponibles en el suelo P1, y las bases de esta selección son diferentes entre plantas. Guisantes y habas seleccionan fuertemente genotipos específicos, pero de manera opuesta. Los nódulos de guisante son colonizados por cepas que poseen un gran conjunto de genes probablemente implicados en la supervivencia en la rizosfera, independientemente del genotipo simbiótico que alberguen, aunque esto debería ser confirmado in situ por métodos transcriptómicos. Las habas restringen la selección a un determinado genotipo simbióticos que no siempre se localiza en el mismo plásmido simbiótico ni con el mismo fondo cromosómico. No se ha podido obtener ninguna conclusión final respecto a vezas, aunque sugerimos que esta planta puede actuar de manera dual, como un hospedador selectivo si el genotipo en cuestión proporciona una ventaja en la supervivencia en la rizosfera (como pasa en la subpoblación vetch ${ }_{B}$ ), o como un hospedador no selectivo, reflejando en los nódulos la diversidad presente en el suelo (como sucede en la subpoblación vetch $\mathrm{A}_{\mathrm{A}}$ ). Esta última es la situación encontrada para lentejas; ninguna de las tres subpoblaciones aisladas de nódulos de lenteja (initial lentil, lentil $A_{A}$ and lentil $_{B}$ ) presenta diferencias significativas entre ellas, independientemente del número de ciclos de selección por la planta, o con la población del suelo inicial. 


\section{LIST OF ABBREVIATIONS}

(Excluding chemical symbols and International Units)

\begin{tabular}{|c|c|}
\hline A & Adenine \\
\hline aa & amino acid \\
\hline aas & Amino acids \\
\hline Ala & Alanine \\
\hline ANI & Average Nucleotide Identity \\
\hline ANIm & ANI-MUMmer \\
\hline A.P & Assimilable Phosphate \\
\hline Arg & Arginine \\
\hline Asp & Aspartate \\
\hline atm & atmosphere \\
\hline BLAST & Basic Local Alignment Search Tool \\
\hline BNF & Biological Nitrogen Fixation \\
\hline bp & Base pair(s) \\
\hline BPKM & Bases Per Kilobase per Million reads \\
\hline bv. & Biovar \\
\hline C & Cytosine \\
\hline $\mathrm{C} / \mathrm{N}$ & Carbon/Nitrogen ratio \\
\hline ca. & circa (approximately) \\
\hline CCaMK & Calmoduline/Calcium-dependent Protein Kinase \\
\hline CDS & Coding DNA Sequence \\
\hline cfu & colony forming unit \\
\hline DNA & Deoxyribonucleic Acid \\
\hline dNTPs & Deoxyribonuclotide triphosphates \\
\hline dpi & days post inoculation \\
\hline EPS & Exopolysaccharide \\
\hline ETI & Effector Triggered Immunity \\
\hline ETS & Effector Triggered Susceptibility \\
\hline$F_{\mathrm{ST}}$ & Wright's fixation index \\
\hline G & Guanine \\
\hline Gln & Glutamine \\
\hline Glu & Glutamate \\
\hline Ile & Isoleucine \\
\hline $\mathrm{kb}$ & kilobases \\
\hline kDa & kilodalton \\
\hline KPS & Capsular Polysaccharide \\
\hline LB & Luria-Bertani broth \\
\hline LCO & Lipochitooligosaccharide \\
\hline Leu & Leucine \\
\hline Log & Logarithm \\
\hline LPS & Lipopolysaccharide \\
\hline Lys & Lysine \\
\hline MAMP & Microbe-Associated Molecular Pattern \\
\hline
\end{tabular}




\begin{tabular}{|c|c|}
\hline $\mathrm{Mb}$ & megabases \\
\hline MPN & Most Probable Number \\
\hline MTI & MAMP Triggered Immunity \\
\hline N's & Mismatches \\
\hline $\mathrm{N}_{\text {ase }}$ Act. & Nitrogenase Activity \\
\hline NCR & Nodule-specific Cysteine-Rich \\
\hline n.d. & no data \\
\hline NF & Nod Factor \\
\hline NFR & Nod Factor Receptor \\
\hline NIN & Nodule Inception \\
\hline No. & number \\
\hline Nod & Presence of nodules \\
\hline NOP & Nodulation Outer Protein \\
\hline nt & Nucleotides \\
\hline${ }^{\circ} \mathrm{C}$ & Degrees (Celsius) \\
\hline$O D_{600 n m}$ & Optical Density at $600 \mathrm{~nm}$ \\
\hline OM & Organic Material \\
\hline ORF & Open Reading Frame \\
\hline PCR & Polymerase Chain Reaction \\
\hline $\mathrm{PE}$ & Paired-End \\
\hline PHA & polyhydroxyalkanoate \\
\hline PHB & Poly-ß-hydroxybutyrate \\
\hline Phe & Phenyalanine \\
\hline ppm & parts per million \\
\hline Pro & Proline \\
\hline PRR & Host Pattern Recognition Receptor \\
\hline pSym & Symbiotic plasmid \\
\hline PVPP & Polyvinylpolypyrrolidone \\
\hline$R$ gene & Resistance gene \\
\hline Ref & reference \\
\hline REP-PCR & Repetitive Extragenomic Palindromic PCR \\
\hline RFLP & Restriction Fragment Length Polymorphism \\
\hline $\mathrm{RL}$ & Rhizobium leguminosarum \\
\hline RIp & Rhizobium leguminosarum bv phaseoli \\
\hline Rlt & Rhizobium leguminosarum bv trifolii \\
\hline Rlv & Rhizobium leguminosarum bv viciae \\
\hline RPKM & Reads Per Kilobase per Million reads \\
\hline rpm & revolutions per minute \\
\hline rRNA & Ribosomal Ribonucleic Acid \\
\hline RTX & Repeat in ToXin \\
\hline SAL & Salinity \\
\hline SD-CDS & Significantly Different Coding DNA Sequence \\
\hline SD-SNP & Significantly Different Single Nucleotide Polymorphism \\
\hline SDS & Sodium Docecyl Sulfate \\
\hline SNP & Single Nucleotide Polymorphism \\
\hline sp. & specie (singular) \\
\hline
\end{tabular}




$\begin{array}{ll}\text { spp. } & \text { species pluralis (plural) } \\ \text { SYMRK } & \text { Symbiosis Receptor-like Kinases } \\ \text { T-N } 2 & \text { Total Nitrogen } \\ \text { T3SS } & \text { Type III secretion system } \\ \text { T4SS } & \text { Type IV secretion system } \\ \text { T6SS } & \text { Type VI secretion system } \\ \text { TBE } & \text { Tris base, Boric Acid and EDTA } \\ \text { tRNA } & \text { transfer Ribonucleic Acid } \\ \text { TS } & \text { Trypticasein Soy } \\ \text { TY } & \text { Tryptone yeast extract } \\ \text { UPGMA } & \text { Unweighted Pair Group Method with Arithmetic Mean } \\ \text { V/v } & \text { volume/volume } \\ \text { Val } & \text { Valine } \\ \text { W/V } & \text { weight/volume } \\ \text { YM } & \text { Yeast Mannitol }\end{array}$





\section{GLOSSARY}

Concepts defined in this Thesis

Absent gene: Gene with a coverage value below 20 RPKM in the initial host subpopulation; or 10 BPKM in the three host-selected subpopulations: initial subpopulation, subpopulation $\mathrm{A}_{\mathrm{A}}$ and subpopulation $_{\mathrm{B}}$.

Conserved gene: Gene with a coverage value above 10 BPKM in the three hostselected subpopulations: initial subpopulation, subpopulation $_{\mathrm{A}}$ and subpopulation $_{\mathrm{B}}$.

Depleted gene: Gene whose coverage values diminished during the mesocosm experiment. Defined as genes that, in both mesocosm host-selected subpopulations (subpopulation $\mathrm{A}_{\mathrm{A}}$ and subpopulation $\mathrm{B}_{\mathrm{B}}$ ), have $\mathrm{a} \log _{2}$ value of fold-change compared to the initial host greater than 2 $\left(\log _{2}\left[\right.\right.$ subpopulation $_{A} /$ initial subpopulation] and $\log _{2}$ [subpopulation ${ }_{B} /$ initial subpopulation]).

Enriched gene: Gene whose coverage values increased during the mesocosm experiment. Defined as genes that, in both mesocosm host-selected subpopulations (subpopulation ${ }_{A}$ and subpopulation ${ }_{B}$ ), have a $\log _{2}$ value of fold-change compared to the initial host greater than 2, (Log $_{2}$ [subpopulation $/$ /initial subpopulation] and $\log _{2}$ [subpopulation $/$ /initial subpopulation] ).

Host selected CDSs: Coding DNA Sequences that have a non-significant average $F_{\mathrm{ST}}$ value ( $<0.1)$ among each host-selected Pool-Seqs: initial subpopulation vs subpopulation $_{A}$; initial subpopulation vs subpopulation $n_{B}$ and subpopulation $_{A}$ vs subpopulation . $_{\text {. }}$

Host subpopulation: Average coverage (RPKM/BPKM) of the four initial host subpopulations: pea, lentil, fava bean and vetch obtained in Chapter 1

Initial host-selected subpopulations: Pool-Seqs obtained from nodules after one step of selection from the four different hosts: Pisum sativum (initial pea), Lens culinaris (initial lentil) Vicia faba (initial fava bean) and V. sativa (initial vetch) obtained in Chapter 1. 
Low-coverage region: Region from the reference genome that has a coverage of Pool-Seq reads significantly lower than the adjacent regions and spans more than 40 genes.

Mesocosm host selected CDSs: Coding DNA Sequences that have a non-significant average $F_{\mathrm{ST}}$ value $(<0.1)$ among each mesocosm host-selected Pool-Seqs: subpopulation $_{\mathrm{A}}$ vs subpopulation $\mathrm{B}_{\mathrm{B}}$.

Mesocosm non-host selected CDSs: Coding DNA Sequences that have highly significant average $F_{\mathrm{ST}}$ value (>0.25) among each mesocosm host-selected Pool-Seqs: subpopulation vs $_{\mathrm{A}}$ subpopulation $_{\mathrm{B}}$.

Mesocosm-selected strains: Strains selected from each mesocosm subpopulation that represent the most abundant $r p o B$ and nodC genotype (Chapter 3 ).

Mesocosm-selected subpopulations: Pool-Seqs obtained from nodules after five cycles of selection from the four different hosts: Pisum sativum $\left(\mathrm{pea}_{\mathrm{A}}\right.$ and pea $_{B}$ ), Lens culinaris (lentil $A_{A}$ and lentil ${ }_{B}$ ) Vicia faba (fava bean ${ }_{A}$ and fava bean $_{B}$ ) and $V$. sativa (vetch and $_{A}$ vetch ${ }_{B}$ ) obtained in Chapter 3.

Non-host selected CDSs: Coding DNA Sequences that have a highly significant average $F_{S T}$ value $(>0.25)$ among each host-selected Pool-Seqs: initial subpopulation vs subpopulation $\mathrm{A}_{\mathrm{A}}$; initial subpopulation vs subpopulation $_{B}$ and subpopulation $_{\mathrm{A}}$ vs subpopulation $\mathrm{B}_{\mathrm{B}}$.

Overrepresented gene: Gene with a coverage value above 200 BPKM in the three host-selected subpopulations: initial subpopulation, subpopulation $\mathrm{A}_{\mathrm{A}}$ and subpopulation $_{B}$.

Overrepresented peak: Region from the reference genome that has a Pool-Seq read coverage value over 200 RPKM/BPKM.

Rhizobia glnll+: Bacteria isolated directly form soil that were only positive for glnll marker.

Rhizobia other: Bacteria isolated directly from soil that lack all the markers analysed, fnrN, glnIl, nodC and nifH.

Rhizobium sp. total: Bacteria isolated directly from soil that were positive for fnrN marker.

Rhizobium sp. non-symbiotic: Bacteria isolated directly from soil that were positive for $f n r N$ but not for nod $C$ neither nifH markers. 
Rhizobium sp. other: Bacteria isolated directly from soil that were positive for fnrN and for either nodC or nifH marker.

Rhizobium sp. symbiotic: Bacteria isolated directly from soil that were positive for $f n r N$, nodC and nifH markers. These were all confirmed in planta.

SD-SNP: Significantly Different Single Nucleotide Polymorphism. SNPs were considered significantly different between two Pool-Seqs if the $F_{\mathrm{ST}}$ value was greater than 0.25 .

SD-CDS: Significantly Different Coding DNA Sequences. CDSs were considered significantly different between two Pool-Seqs if the average $F_{\mathrm{ST}}$ value was greater than 0.25 . 



\section{$\underline{\text { TABLE OF CONTENTS }}$}

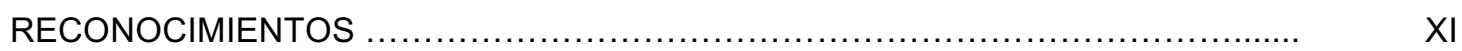

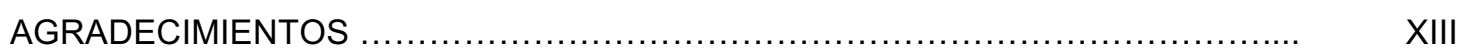

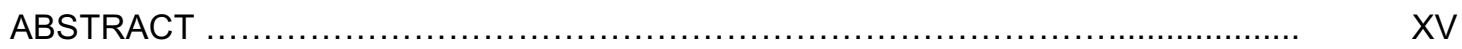

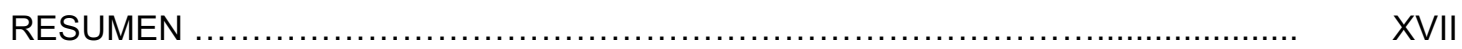

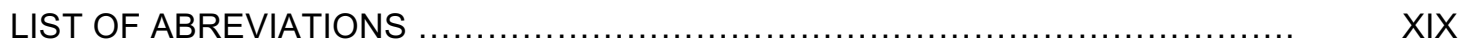

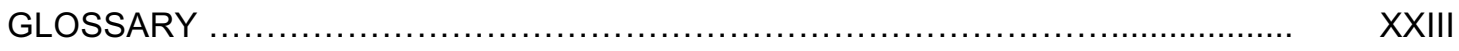

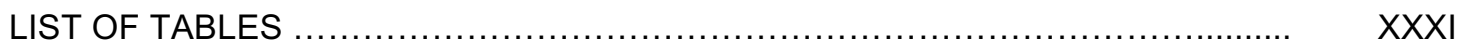

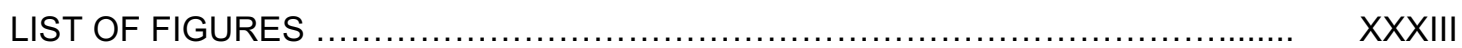

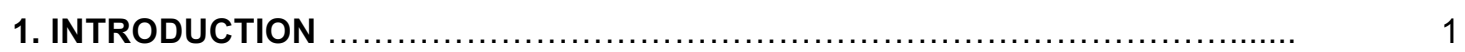

1.1 RELEVANCE OF BIOLOGICAL NITROGEN FIXATION .......................... 3

1.2 THE RHIZOBIUM-LEGUME SYMBIOSIS …................................ 5

1.3 HOST SPECIFICITY ............................................................

1.4 Rhizobium leguminosarum ................................................ 12

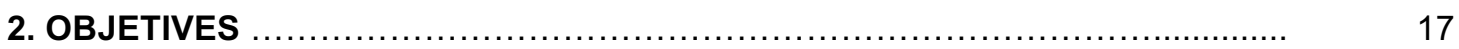

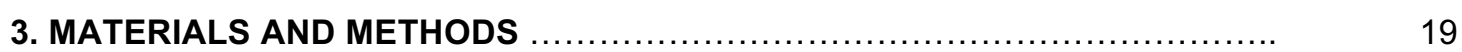

3.1. SITE AND SOIL CHARACTERISTICS ....................................... 21

3.2. PLANT MATERIAL ........................................................ 22

3.3. BACTERIAL STRAINS ................................................ 22

3.4. CULTURE MEDIA AND GROWTH CONDITIONS .............................. 25

3.5. OLIGONUCLEOTIDES ................................................... 25

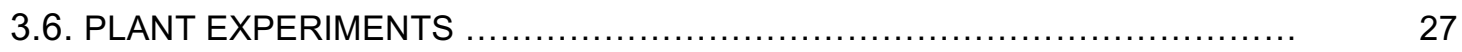

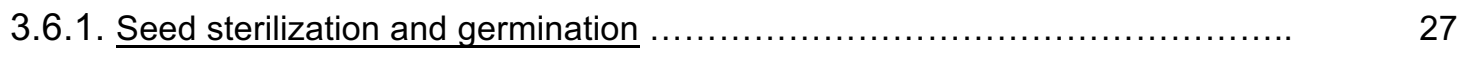

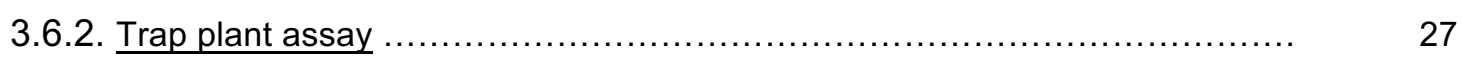

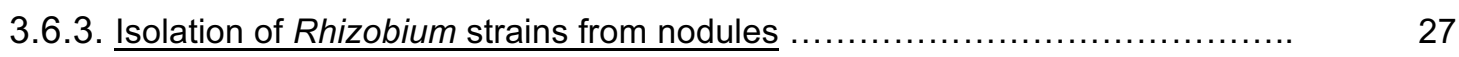

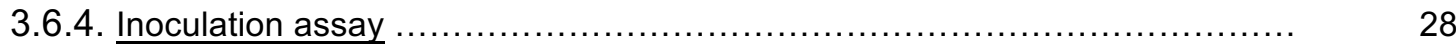

3.6.5. Mesocosm experiment …................................................ 28 
3.7. MICROBIOLOGICAL TECHNIQUES ...................................... 29

3.7.1. Preparation of cultures for Pool-Seq ......................................... 29

3.7.2. Isolation of rhizobial strains from soil ….................................... 29

3.8. NUCLEIC ACID METHODS ................................................... 30

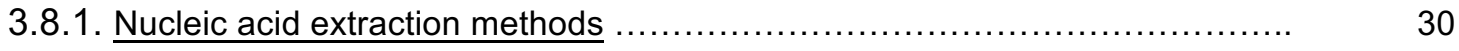

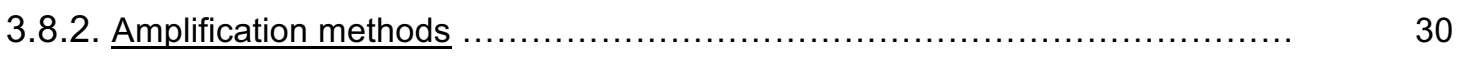

3.8.2.1. Standard PCR ............................................................. $\quad 30$

3.8.2.2. Electrophoresis ............................................................ 30

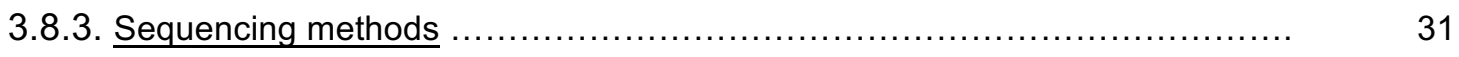

3.8.3.1. Sanger .................................................................. 31

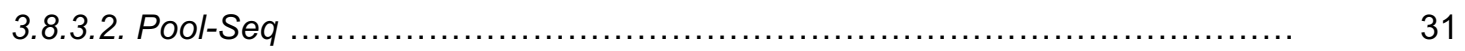

3.8.3.3. Whole genome sequencing ...............................................

3.9. BIOINFORMATIC METHODS …......................................... 31

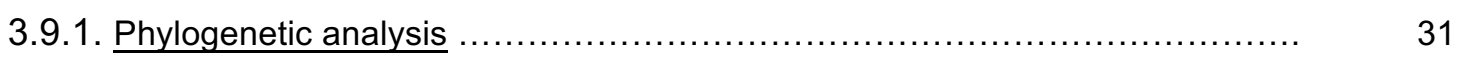

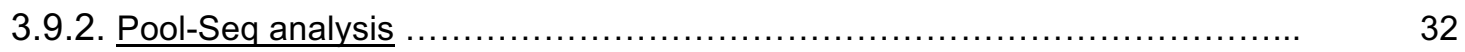

3.9.3. Genome Assembly .................................................... 33

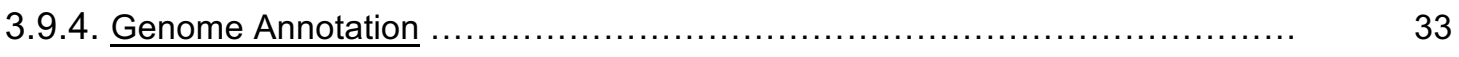

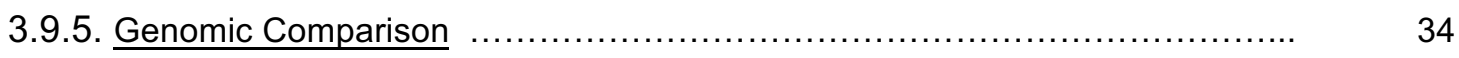

4. CHAPTER 1: Population Genomics Analysis of Legume Host Preference for Specific Rhizobial Genotypes in the Rhizobium leguminosarum bv. viciae

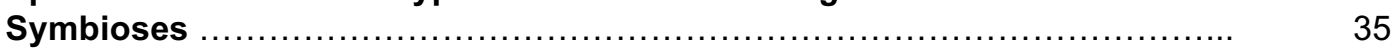

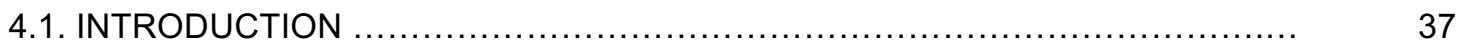

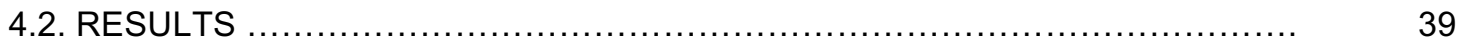

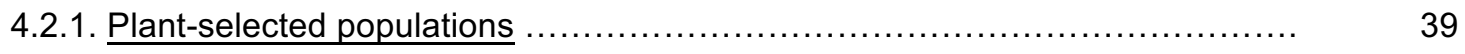

4.2.2. Next generation sequencing of pooled, plant-selected rhizobial populations .... $\quad 39$

4.2.3. Presence of sequences from the $R$. leguminosarum bv. viciae reference

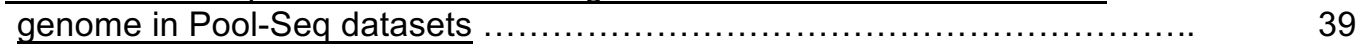

4.2.4. Sequence polymorphisms in specific regions of Pool-Seq datasets .............. 42

4.2.4.1. 16S rRNA-23S rRNA region ...............................................

4.2.4.2. nod cluster ........................................................... 44

4.2.5. Genome-wide sequence polymorphisms in Pool-Seq datasets ................. 46

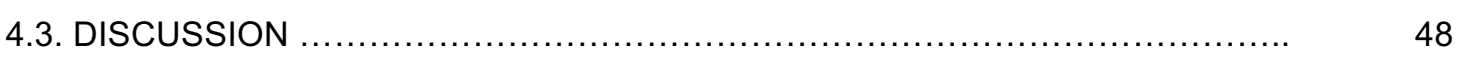

4.4. SUPPLEMENTARY MATERIAL ............................................ 52 
5. CHAPTER 2. Population Genomic Characterization of Native Rhizobium

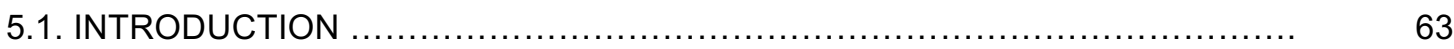

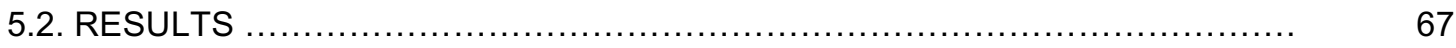

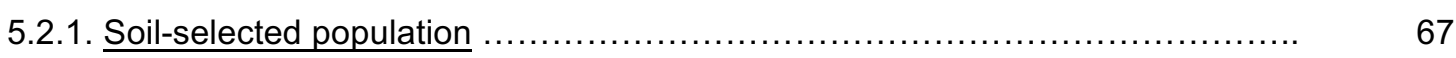

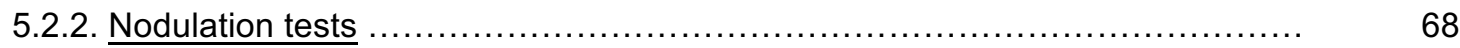

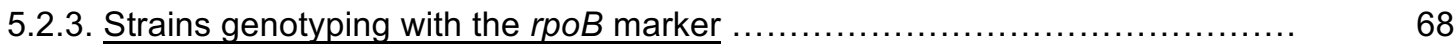

5.2.4. Next generation sequencing of the pooled soil rhizobial population ............. 72

5.2.5. Presence of sequences from the $R$. leguminosarum bv. viciae 3841 reference genome in the soil Pool-Seq dataset.....

5.2.6. Differential abundance of $R$. leguminosarum bv. viciae 3841 reference genome sequences in soil Pool-Seq and host Pool-Seqs 3 7 7 8

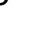

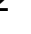

5.2.7. Genome-wide sequence polymorphisms in Pool-Seq datasets .................. 75

5.2.8. Sequence polymorphisms in specific regions of Pool-Seq datasets .............. 77

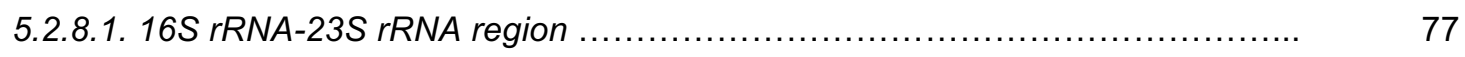

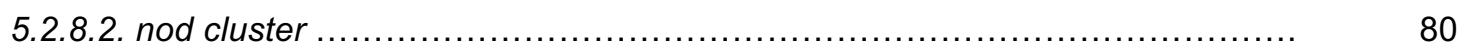

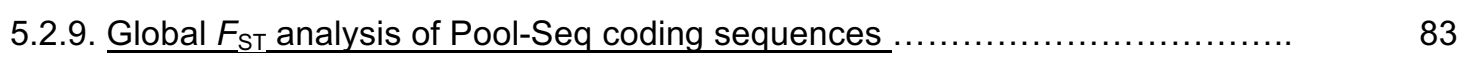

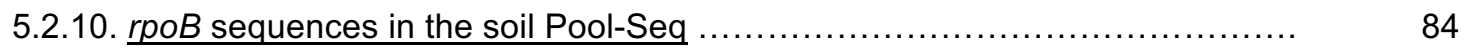

5.2.11. Pool-Seq analysis using other, closely related genomes as reference .......... 85

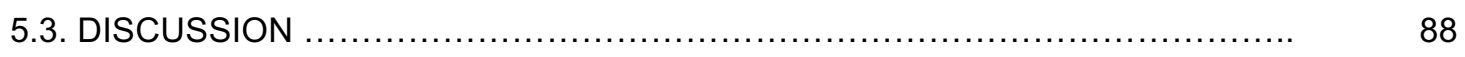

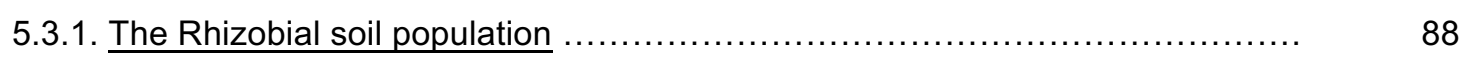

5.3.2. Host selection of genotypes from those available in the soil .................... 91

5.4. SUPPLEMENTARY MATERIAL ........................................... 96

6. CHAPTER 3. Mesocosm Analysis of Rhizobium leguminosarum Genotype Selection by Different Fabeae Legume Hosts .............................. 105

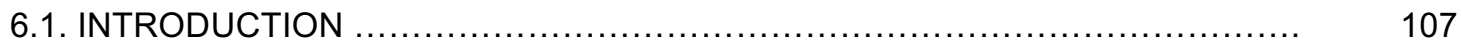

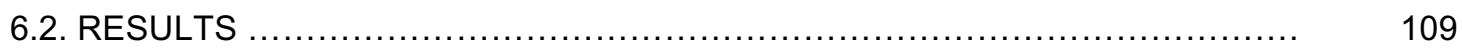

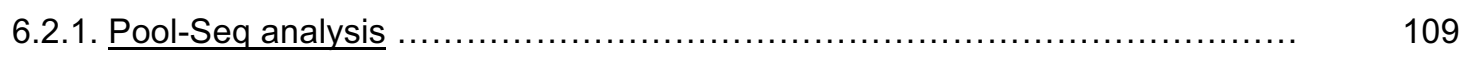

6.2.1.1. Mesocosm-selected rhizobial populations ..................................... 109

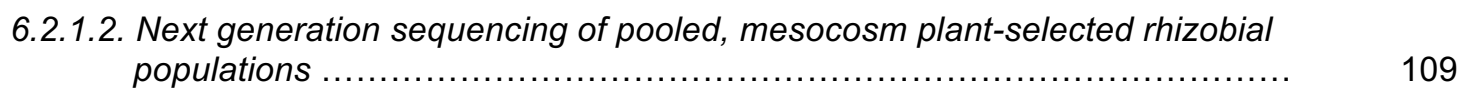

6.2.1.3. Presence of sequences from the R. leguminosarum bv. viciae 3841 reference genome in mesocosm plant-selected rhizobial populations ......... 110

6.2.1.4. Conservation of genes from the $R$. leguminosarum bv. viciae 3841
reference genome in plant host-selected subpopulations ................... 118

6.2.1.4.1. Conserved genes ..................................................... 118

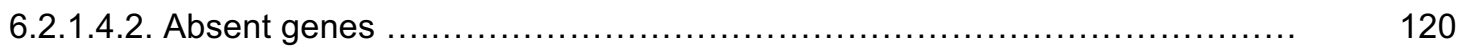

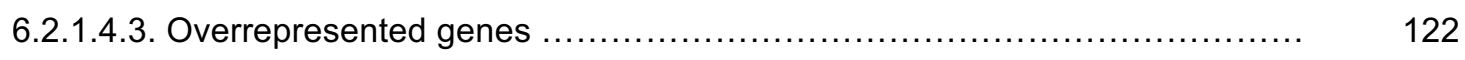


6.2.1.5. Differential coverage of $R$. leguminosarum bv. viciae 3841 reference genome sequences in host-selected Pool-Seqs

6.2.1.5.1. Genes exclusively enriched after five cycles of host selection ............... 123

6.2.1.5.2. Genes exclusively depleted after five cycles of host-selection .............. 125

6.2.1.6. Genome-wide sequence polymorphisms in Pool-Seq datasets ............... 127

6.2.1.7. Global F $F_{S T}$ analysis of Pool-Seq coding sequences ........................... 129

6.2.1.7.1. Host-selected CDSs (intra-host comparisons) .......................... 130

6.2.1.7.2. Non-host selected CDSs (intra-host comparisons) ........................ 131

6.2.1.7.3. Mesocosm host-selected CDSs (intra-host comparisons) ................... 131

6.2.1.7.4. Mesocosm non-host selected CDSs (intra-host comparisons) .............. 132

6.2.1.8. $F_{S T}$ analysis of specific coding sequences in Pool-Seq datasets ............... 132

6.2.1.8.1. Housekeeping marker regions ................................... 133

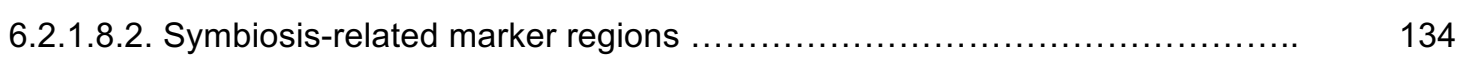

6.2.1.8.3. Rhizospheric marker regions ......................................... 136

6.2.1.8.4. Differential regions among host-selected subpopulations (inter-host

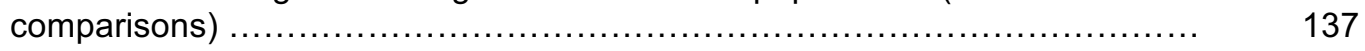

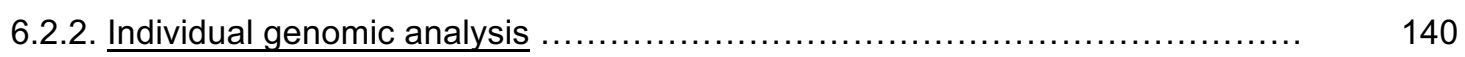

6.2.2.1. Selection of genotypes .................................................... 140

6.2.2.2. Cross-inoculation assay and nitrogenase activity .......................... 142

6.2.2.3. Next generation sequencing of mesocosm-selected strains ................... 142

6.2.2.4. Mesocosm-selected strains adequately represent their corresponding PoolSeq subpopulations ................................................. 144

6.2.2.5. Average Nucleotide Identity (ANI) of mesocosm-selected strains ............. 144

6.2.2.6. Symbiotic clusters: Synteny and diversity ............................. 145

6.2.2.7. Symbiotic region organization ...................................... 154

6.2.2.8. Presence and diversity of "mesocosm-related" genes ........................ 155

6.2.2.9. Exclusive genes in plant host mesocosm-selected strains .................. 157

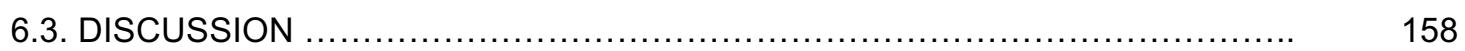

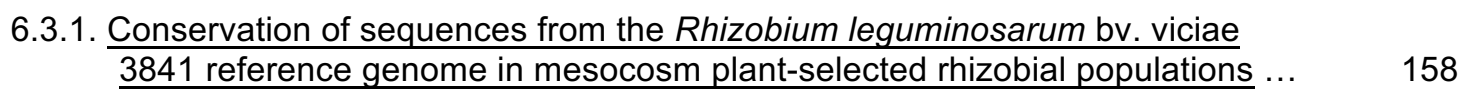

6.3.2. Genome-wide sequence polymorphisms in mesocosm Pool-Seq datasets ..... 160

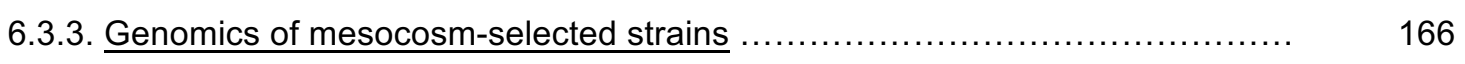

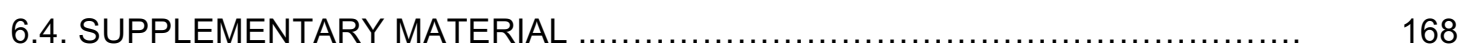

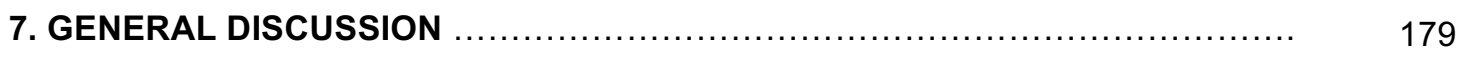

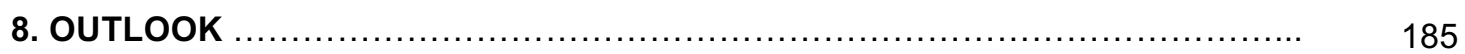

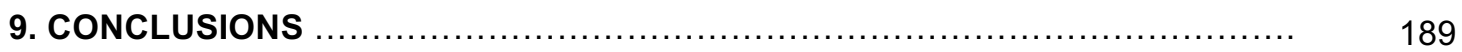

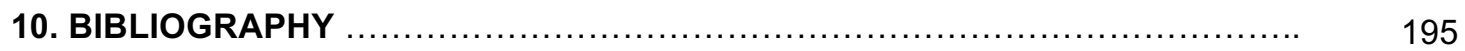




\section{LIST OF TABLES}

Table M.1. Soil chemical analysis for each soil previous (Bulk) and after five cycles of host-selection.

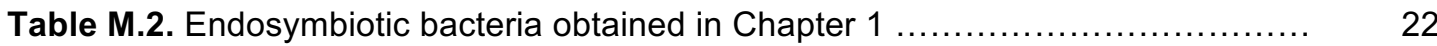

Table M.3. Bacteria obtained in Chapter 2 ............................................ 23

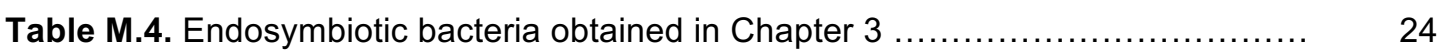

Table M.5. List of primers used in this Thesis ........................................ 26

Table 1.1. Average coverage (RPKM, Reads Per Kilobase per Million reads) of each reference genome (Rhizobium leguminosarum bv. viciae 3841) replicon by Pool-Seq DNA samples of the four plant-selected populations

Table 1.2. Genome-wide abundance of single nucleotide polymorphism in Pool-Seq DNA samples of the four plant-selected populations

Supplementary Table 1.S1. Pairwise comparison of SNPs among plant-selected rhizobial subpopulations and with the reference genome in the nod region

Supplementary Table 1.S2. Pairwise comparison of SNPs among plant-selected rhizobial subpopulations and with the reference genome for the whole genome

Supplementary Table 1.S3. Euclidean distances among plant-selected rhizobial subpopulations sequences derived from genome-wide SNP frequency dissimilarities

Table 2.1. Amount of different soil bacteria isolated from $P 1$ Dijon soil

Table 2.2. Amount of different rhizobial genotypes as result of the markers screening

Table 2.3. Cross inoculation assay results

Table 2.4. Average coverage (RPKM) of each reference genome (Rhizobium leguminosarum bv. viciae 3841) replicon by soil Pool-Seq sample datasets

Table 2.5. Genome-wide abundance of significantly different single nucleotide polymorphisms in Pool-Seq DNA samples respect to soil Pool-Seq

Table 2.6. $F_{\text {ST }}$ values for SD-SNPs in the rRNA region

Table 2.7. Nucleotide frequencies of SD-SNPs in the rRNA region .... 79

Table 2.8. $F_{\mathrm{ST}}$ values for SD-SNPs in the nod cluster

Table 2.9. Nucleotide frequencies of SD-SNPs in the nod cluster

Table 2.10. Exclusive selected genes for all subpopulation

Table 2.11. Reference genome characteristics

Table 2.12. ANIm values among references genomes

Table 2.13. Percentage of aligned reads in each $R$. leguminosarum bv. viciae reference genome for each Pool-Seq

Table 2.14. Genome-wide abundance of significantly different single nucleotide polymorphisms in Pool-Seq DNA samples respect to soil Pool-Seq expressed in percentage

Supplementary Table 2. S1. Screening genotype and nodulation phenotype of soilselected population

Supplementary Table 2.S2. Exclusive selected genes for each host-selected subpopulation

Table 3.1. Average coverage (BPKM) of each reference genome (Rhizobium leguminosarum bv. viciae 3841) replicon by all Pool-Seq sample datasets 
Table 3.2. Presence and absence of overrepresented chromosome peaks for each mesocosm host-selected subpopulation

Table 3.3. Number of genes that were significantly different between each mesocosm Pool-Seq and the average of initial host Pool-Seqs

Table 3.4. Percentage of positions that had a highly significant $F_{\mathrm{ST}}$ value $(\geq 0.25)$ for each Pool-Seq pairwise comparison

Table 3.5. Non-informative CDSs present in all Pool-Seqs

Table 3.6. Cross inoculation results of the seven selected strains in four plant hosts ..

Table 3.7. Genome sequence assembly statistics for the seven selected strains

Table 3.8. Genome statistics for the seven selected strains

Table 3.9. Comparison of mesocosm host-specific Pool-Seqs with their corresponding host-selected reference genome

Table 3.10. nodF- $D$ intergenic region sizes

Table 3.11. Presence and absence of mesocosm-related genes

Supplementary Table 3.S1. Non significant CDSs present in all Pool-Seqs 


\section{LIST OF FIGURES}

Figure I.1 Nitrogen Cycle

Figure I. 2. Nodule Development

Figure I. 3. Host Specificity and Symbiosis

Figure I. 4. Genome synteny between Rhizobium leguminosarum bv. viciae 3841 and $R$. leguminosarum bv. viciae UPM791

Figure I.5. Rhizobium leguminosarum bv. viciae legume hosts

Figure 1.1. Conservation of $R$. leguminosarum bv. viciae 3841 reference genome sequences in the $\mathrm{P} 1$ soil rhizobial population

Figure 1.2. Conservation and diversity of the 16S-23S rRNA genomic region in the four plant-selected rhizobial populations

Figure 1.3. Conservation and diversity of the nod region in the four plant- selected rhizobial populations

Supplementary Figure 1.S1. Conservation of reference genome chromosome in the four plant-selected rhizobial populations

Supplementary Figure 1.S2. Conservation of small plasmid replicons from the reference genome in the four plant-selected rhizobial populations

Supplementary Figure 1.S3. Conservation of reference genome plasmid pRL9 in the four plant-selected rhizobial populations

Supplementary Figure 1.S4. Conservation of reference genome plasmid pRL10 in the four plant-selected rhizobial populations

Supplementary Figure 1.S5. Conservation of reference genome plasmid pRL11 in the four plant-selected rhizobial populations

Supplementary Figure 1.S6. Conservation of reference genome plasmid pRL12 in the four plant-selected rhizobial populations

Supplementary Figure 1.S7. Two-dimensional representation of genome- wide SNP dissimilarities among plant-selected rhizobial populations and the reference genome

Figure 2.1. Phylogenetic tree based on partial ( $447 \mathrm{nt}) r p o B$ sequences showing the relationship of $\mathrm{P} 1$ soil isolates and related species within the order Rhizobiales

Figure 2.2. Phylogenetic tree based on partial ( $447 \mathrm{nt}) r p o B$ sequences showing the relationship of a subsample of $\mathrm{P} 1$ soil isolates (N-Z; see Fig. 2.1) and related species within the genus Rhizobium

Figure 2.3. Conservation of the $R$. leguminosarum bv. viciae 3841 reference genome in the $\mathrm{P} 1$ soil rhizobial population

Figure 2.4. Heatmap representation of gene conservation in host and soil Pool-Seqs along the four larger replicons in the reference genome

Figure 2.5. Conservation and diversity of the 16S-23S rRNA genomic region in the four plant-selected rhizobial populations and soil population

Figure 2.6. Conservation and diversity of the nod cluster symbiotic region in the four plant-selected rhizobial populations and soil population

Figure 2.7. Shared and exclusively SD-CDSs for each host-selected subpopulations based on $F_{\mathrm{ST}}$ pairwise values respected to soil population

Figure 2.8. Distribution of polymorphic sites in the partial rpoB sequence (447 nt) used for genotyping that are present in the Pool-Seq and Sanger soil datasets 
Supplementary Figure 2.S1. Phylogenetic tree based on partial (447 nt) rpoB (A) and near complete $(1,287 \mathrm{nt}) 16$ rRNA sequences showing the relationship of selected $\mathrm{P} 1$ soil isolates and related species within the order Rhizobiales

Figure 3.1. Conservation of the chromosome of $R$. leguminosarum bv. viciae 3841 reference genome in the mesocosm plant-selected subpopulations

Figure 3.2. Conservation of the symbiotic plasmid pRL10 of $R$. leguminosarum bv. viciae 3841 reference genome in the mesocosm plant-selected subpopulations

Figure 3.3. Conservation of the plasmid pRL8 of $R$. leguminosarum bv. viciae 3841 reference genome in the mesocosm plant-selected subpopulations

Figure 3.4. Conservation of the plasmid pRL7 of $R$. leguminosarum bv. viciae 3841 reference genome in the mesocosm plant-selected subpopulations

Figure 3.5. Venn diagrams of conserved genes among host-selected subpopulations ..

Figure 3.6. Venn diagrams of absent genes among host-selected subpopulations

Figure 3.7. Venn diagrams of overrepresented genes for each host-selected subpopulation

Figure 3.8. Venn diagrams of genes exclusively enriched after five cycles of host selection among mesocosm host-selected subpopulations

Figure 3.9. Venn diagrams of genes exclusively depleted after five cycles of host selection among mesocosm host-selected subpopulations

Figure 3.10. Two-dimensional representation of global $F_{\mathrm{ST}}$ average

Figure 3.11. Two-dimensional representation of $F_{\mathrm{ST}}$ for $16 \mathrm{~S}$ and $23 \mathrm{~S}$ rRNA genes .....

Figure 3.12. Two-dimensional representation of $F_{\mathrm{ST}}$ for some housekeeping markers .

Figure 3.13. Two-dimensional representation of $F_{\mathrm{ST}}$ for some symbiosis-related markers

Figure 3.14. Two-dimensional representation of $F_{\mathrm{ST}}$ for some rhizospheric markers ....

Figure 3.15. Two-dimensional representation of $F_{\mathrm{ST}}$ for differential regions (inter-host)

Figure 3.16. Two-dimensional representation of $F_{\mathrm{ST}}$ for differential genes (inter-host) ..

Figure 3.17. $r p o B$ and nod $C$ mesocosm genotypes

Figure 3.18. Genomic similarity among mesocosm-selected strains

Figure 3.19. Genomic organization, synteny and diversity of nif cluster

Figure 3.20. Genomic organization, synteny and diversity of rhi cluster

Figure 3.21. Genomic organization, synteny and diversity of nod cluster

Figure 3.22. Genomic organization, synteny and diversity of nif-fix cluster

Figure 3.23. Genomic organization, synteny and diversity of fix cluster

Figure 3.24. Genomic organization and synteny of the symbiotic region from Rhizobium leguminosarum bv. viciae 3841 type mesocosm-selected genomes

Figure 3.25. Genomic organization and synteny of the symbiotic region from Rhizobium leguminosarum bv. viciae UPM791 type mesocosm-selected genomes .....

Supplementary Figure 3.S1. Conservation of the plasmid pRL12 of $R$. leguminosarum bv. viciae 3841 reference genome in the mesocosm plant-selected subpopulations

Supplementary Figure 3.S2. Conservation of the plasmid pRL11 of $R$. leguminosarum bv. viciae 3841 reference genome in the mesocosm plant-selected subpopulations 
Supplementary Figure 3.S3. Conservation of the plasmid pRL9 of $R$. leguminosarum bv. viciae 3841 reference genome in the mesocosm plant-selected subpopulations ....

Supplementary Figure 3.S4. Phylogenetic trees based on housekeeping and chromosomal "symbiosis-related" genes

Supplementary Figure 3.S5. Phylogenetic trees based on "rhizosphere" and mesocosm-selected genes....

Supplementary Figure 3.S6. Phylogenetic trees based on mesocosm-selected genes 

1. INTRODUCTION 



\subsection{RELEVANCE OF BIOLOGICAL NITROGEN FIXATION}

Nitrogen ( $\mathrm{N}$ ), in addition to water, is the nutrient required in highest amount by plants (White and Brown, 2010). N comprises 1.5-2\% of plant dry matter and approximately $16 \%$ of total plant protein fraction (Frink et al., 1999). Its availability limits growth and plant development and therefore crop yield (Gutiérrez, 2012). Despite its abundance, most $\mathrm{N}$ in the biosphere exists in the biologically inaccessible form of atmospheric $\mathrm{N}$ gas $\left(\mathrm{N}_{2}\right)$ (Canfield et al., 2010) (Figure I.1). In agricultural areas of low $\mathrm{N}$ availability, it is supplied to crops as fertilizer to achieve greater yields (White and Brown, 2010). At the beginning of the $20^{\text {th }}$ century Fritz Haber developed an industrial process to reduce $\mathrm{N}_{2}$ to ammonia $\left(\mathrm{NH}_{3}\right)$ by combining it with hydrogen gas in the presence of iron. Later on, in 1914 Karl Bosch established the first large ammonia manufacturing factory (Frink et al., 1999). This process, known as Haber-Bosch, is energy intensive, needing high pressures (150-300 atmospheres, atm) and temperatures (400-500 Celsius grades, ${ }^{\circ} \mathrm{C}$ ) conditions. The production and use of $\mathrm{N}$ fertilizers on an industrial scale increases global agricultural productivity. The estimation of the numbers of humans supported per hectare of arable land increased from 1.9 to 4.3 between 1908-2008 (Erisman et al., 2008). From 1960 to 1995 the $\mathrm{N}$ fertilizers applied to agricultural crops increased 7-fold, whereas the global yield increase was just 2.4-fold (Tilman et al., 2002). In most intensive agricultural crops, like maize, ca. $50-75 \%$ of $\mathrm{N}$ applied is not used by plants and is leached into the soil (Francis et al., 1993). Due to its solubility, necessary for easy uptake and assimilation by plants, ammonia and urea from $\mathrm{N}$ fertilizers, can run off into the surface water or flow into the groundwater (Hirel et al., 2011). Water contaminated by nitrate is not potable and at high concentrations can be harmful for human health (Bryan and van Grinsven, 2013). The use of fertilizers contributes to environmental pollution (White and Brown, 2010), not only by leaching into the soil, but also by the production of greenhouse gasses during their synthesis (Galloway et al., 2008). Therefore, for commercial and environmental reasons, the use of $\mathrm{N}$ fertilizers should be exercised with care, and consequently, sustainable fertilizer management is required for future crop production (White and Brown, 2010).

For centuries legumes have been use in crop rotations to incorporate $\mathrm{N}$ into agricultural systems avoiding the need for fertilization (Geddes et al., 2015). This phenomenon occurs because of the symbiotic interaction with the diazotrophic soil bacteria collectively known as rhizobia. These bacteria infect legume roots, form root nodules and fix $\mathrm{N}_{2}$ into ammonia using the nitrogenase enzyme within those nodules (Oldroyd et al., 2011). Nitrogenase only occurs in prokaryotes, and $\mathrm{N}$-fixing symbioses 
are largely restricted to legumes (Young, 1992, Werner et al., 2014). As much as 21.5 tonnes $\mathrm{N}$ is fixed annually by rhizobia in association with legumes (Curatti and Rubio, 2014), and the energy cost of $\mathrm{N}$-fixation in legumes is lower than the cost of an equivalent production of synthetic $\mathrm{N}$ fertilizers (Hirel et al., 2011). As this $\mathrm{N}$-fixation occurs in the root nodule within the plant, this symbiotic partnership commands a molecular conversation that guarantees specificity and guides the co-development of both organisms during nodule formation (Price et al., 2015).

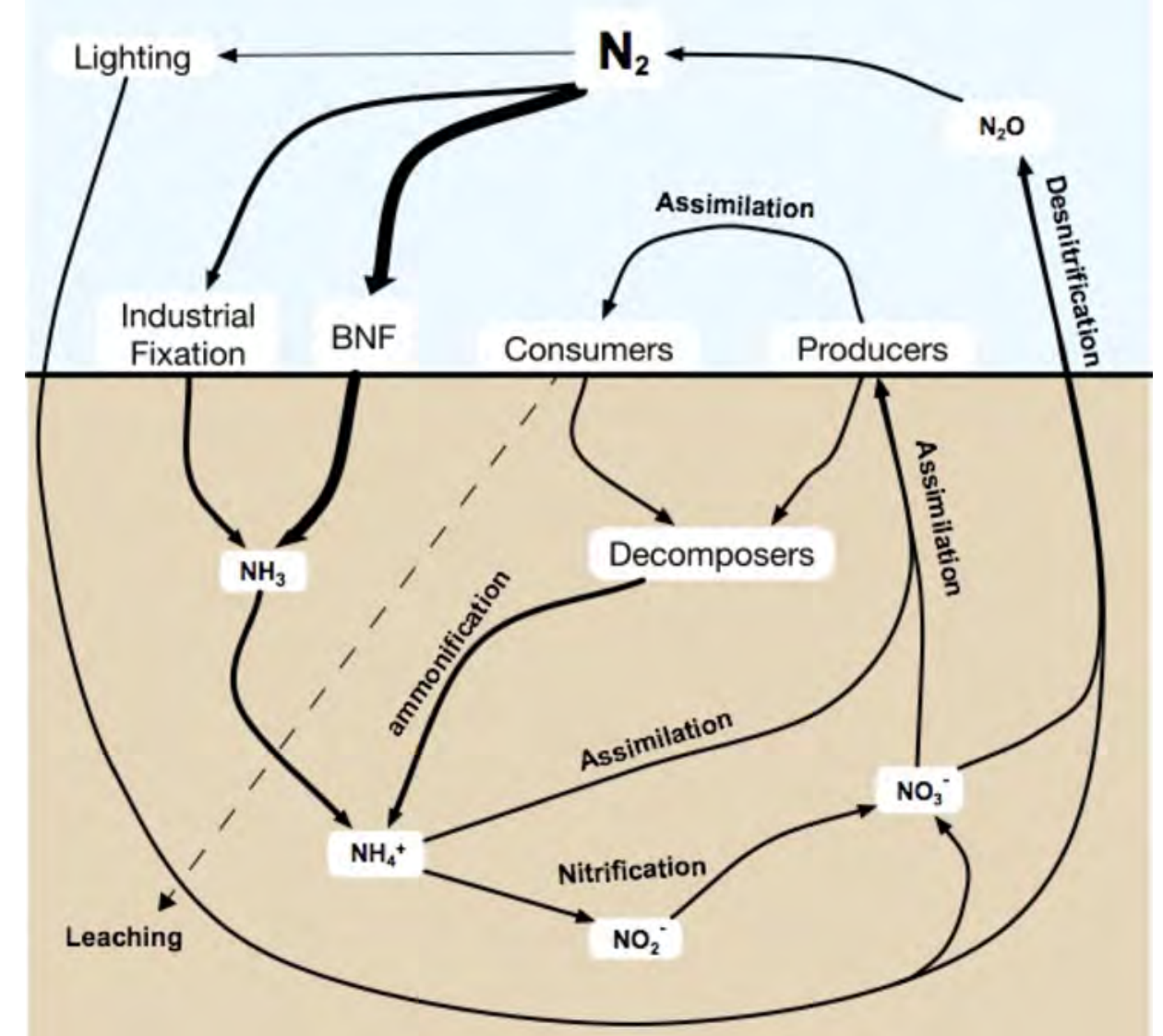

Figure I.1. Nitrogen Cycle. Schematic representation of the biogeochemical nitrogen cycle, adapted from Friedland et al. (2012) 


\subsection{THE RHIZOBIUM-LEGUME SYMBIOSIS}

Biological nitrogen fixation (BFN) is the process whereby $\mathrm{N}_{2}$ is fixed into $\mathrm{NH}_{3}$ by diazotrophic bacteria, and in legumes symbiotic rhizobia carry out BNF within root nodules. Legumes are plants that belong to the family Fabaceae, which is the second most important crop family after the Poaceae. Legumes are widely distributed and include herbaceous plants, shrubs and trees. Grain legumes account for $27 \%$ of world crop production and provide one-third of the human dietary protein requirement (Smýkal et al., 2015). Rhizobia that establish symbioses with legumes are Gramnegative bacteria that belong to the orders Rhizobiales (Alphaproteobacteria), Burkholderiales (Betaproteobacteria) (Sawada et al., 2003) and the genus Pseudomonas (Gammaproteobacteria) (Shiraishi et al., 2010, Ibáñez et al., 2009, Benhizia et al., 2004). Rhizobia persist in soil for years even in the absence of their legume host, due to their ability of survive around the roots of different plants (Downie, 2010).

The development of $\mathrm{N}$-fixing nodules is a complex multistep process that begins with chemotaxis of the bacterium towards the root (Figure I.2, 1). Legumes secrete compounds, such as the phenolic flavonoids and isoflavonoids, that diffuse across the bacterial membrane passively (Recourt et al., 1989). These phenolic compounds are recognized, usually by NodD transcription factor, which in turn binds to conserved promoter regions, known as nod boxes, and induce expression of several genes (Figure I.2, 2). Many of these genes are involved in the biosynthesis of Nod factors (NFs), lipochitooligosaccharide (LCO) molecules, that are key determinants of host specificity (Downie, 2010). NFs are recognised by plant LysM receptor-like kinases (Oldroyd, 2013) and this interaction triggers a signal transduction pathway in host roots inducing major morphological and biochemical changes (Oldroyd and Downie, 2008). As a result of this signal exchange, host root hairs curl, trapping rhizobia in a structure called "shepherds-crook". The rhizobia continue growing and form an infection focus; the constant synthesis of NFs stimulates the development of a membrane and cell wall in growth. The uninterrupted growth of the bacteria at the tip of this narrow tube develops the infection thread (Figure I.2, 3). Infection threads extend between cells, branch and ramify while they develop until they reach the growing meristem, where the bacteria are delivered into the nodule cells by an endocytic process (Figure I.2, 4). Bacteria are surrounded by the plant membrane, forming an organelle called symbiosome, within which the rhizobia differentiate to a state called bacteroid, where the genes required for $\mathrm{N}$-fixation are expressed (Figure I.2, 5) (Downie, 2010). 


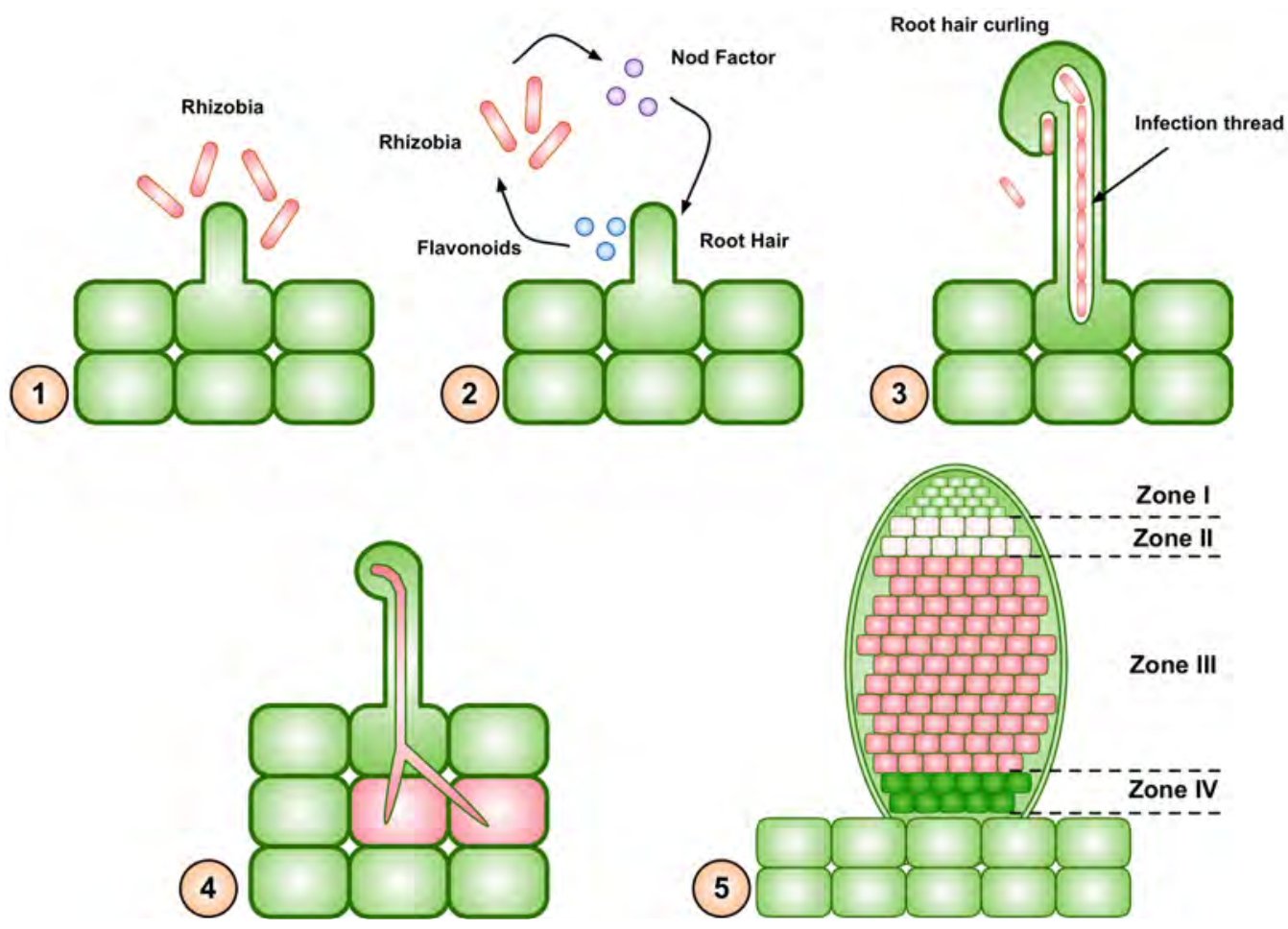

Figure I.2. Nodule development. 1) Chemotaxis of rhizobia towards roots. 2) Signal exchange between rhizobia and legumes. 3) Root hair curling and infection thread formation. 4) Infection thread extension. 5) Nodule formation. Adapted from Deakin and Broughton (2009) and Oldroyd (2013)

Legumes form two types of nodules, determinate and indeterminate. Determinate nodules, such as those from Lotus japonicus and Glycine max, are globose because they develop a spherical meristem that works until the formation of the nodule primordium; infected cells develop more or less synchronously up to the $\mathrm{N}$-fixation stage. Determinate nodules contain bacteroids that are similar in size to the free-living bacteria. By contrast, indeterminate nodules, such as those from Medicago truncatula and Pisum sativum, are elongated due to the presence of a persistent meristem at their apex. In a longitudinal section these nodules show different regions: a meristematic zone (I), an infection zone (II), a nitrogen-fixation zone (III), and a senescent zone (IV) that usually appears after 5 weeks of inoculation (Figure I.2. 5). These nodules present strongly elongated and usually branched bacteroids. The differentiation of bacteria into bacteroids implies dramatic changes in gene expression, cell surface structure and metabolic activities. These changes meet the low oxygen environment conditions needed by nitrogenase to work (Popp and Ott, 2011, Vasse et al., 1990, Kereszt et al., 2011, Patriarca et al., 2002). 


\subsection{HOST SPECIFICITY}

Host specificity is a concept that describes the association between the host and the microbe and is defined as "the partiality to infecting one or a group of defined host". Host specificity is not only related with parasitic interactions, but also with mutualistic and commensalistic associations, therefore the concept could be modified as "the nature and extent of microbial adaptation to one or more particular hosts". Host specificity can be measured at two levels: interspecific and intraspecific. At an interspecific level the range of specialization is of one species over another, while at intraspecific level, the extent of hosts is within a species. Microorganisms present factors or determinants that are needed to associate with a certain host, and are defined as host specificity factors. The classic example of host specificity determinants is NFs in the rhizobia-legume symbiosis, but these are not the only determinants during this interaction (Kirzinger and Stavrinides, 2012, Mandel, 2010, Jorrín and Imperial, 2015b).

Host specificity in symbiosis can occur at early stages, as in the flavonoid recognition, but also in late states during the nitrogen fixation. From a basic perspective, specificity is quite similar to host-pathogen interactions (see below); but from an application point of view, deciphering the molecular mechanisms that underlie this specificity can contribute to crop yield improvement (Wang et al., 2012), since less compatible symbionts are present in domesticated legume species (Mutch and Young, 2004). Understanding the key factors that affect symbiosis specificity will allow us to engineer rhizobial strains or to manipulate crop varieties in order to enhance the symbiosis, and hence $\mathrm{N}$-fixation. (Wang et al., 2012).

The first step of host specificity in the rhizobia-legume symbiosis occurs in the molecular dialogue between plant flavonoids and rhizobia. Legume root exudate a large number of compounds: amino acids, organic acids, aromatic acids, sugar, polyols, phosphates, (Colebatch et al., 2004) and also phenolic compounds and flavonoids (Broughton et al., 2003). Flavonoids are polyphenolic compounds produced as a result of the secondary plant metabolism, and can be released into the rhizosphere either passively (by root injury, root decomposition or root turnover) or actively (by root exudation). In the rhizosphere flavonoids have different roles, such as plant defence, allelopathy, root growth regulation and initiation of the symbiosis (Cesco et al., 2012, Shaw et al., 2006). The exudation of flavonoids by legumes is continuous, but the concentration of flavonoids increases when a compatible bacterium is found (Lira et al., 2015). Once in the rhizosphere, flavonoids act as chemoattractants toward the root, and passively diffuse across the bacterial membrane (Cooper, 2004, Wang et 
al., 2012). Flavonoids mediate the activation of NodD, a LysR-like transcriptional regulator, but no direct biochemical interaction with it has been shown. However, an indirect interaction has been demonstrated, as flavonoids stimulate the DNA binding affinity of NodD to nod gene promoters (nod boxes) (Peck et al., 2006). NodD transcriptional factors from different rhizobia respond to different groups of flavonoids, and no correlation has been demonstrated between the number of flavonoids a given NodD responds to and the host range of the bacterium. As an example, Rhizobium sp. NGR234 presents a very wide host range and responds to a wide variety of flavonoids, whereas Rhizobium leguminosarum bv. viciae (RIv) has a narrow host range, but responds as well to a high number of different flavonoids (Cooper, 2007). Even so, most legumes only excrete a certain type of flavonoids that are able to activate certain NodD transcriptional factors, and this specific recognition is defined as the first checkpoint in the rhizobium-legume dialogue (Figure I.3, A) (Wang et al., 2012). For instance, NodD1 transferred from the broad host-range bacterium Rhizobium sp. NGR234 to Ensifer meliloti or R. leguminosarum bv. trifolii (RIt), allows these bacteria to form nodules in Macroptilium atropurpureum and Parasponia andersonii (Bassam et al., 1986, Horvath et al., 1986, Bender et al., 1988). Another example is the one describe by Mclver et al. (1989) where point mutations in nodD from Rlt extend the host range of this bacterium from clover to $P$. andersonii, through the newly acquired ability of NodD to induce nod genes in the absence of flavonoids. It was shown that the ability of a group of flavonoids to promote nod gene expression is dependent on the source of NodD (Peck et al., 2006).

The second step of host specificity occurs between NFs and NF Receptors (NFRs) (Figure I.3, B). As a consequence of an appropriate flavonoid induction, rhizobia synthesize NFs. All NFs share the same backbone, composed by $\mathrm{N}$-acetyl glucosamine oligosaccharides, but differ in additional modifications along the main structure. The NF backbone is synthesized by the products of the nodABC genes, with a fatty acyl chain at the non-reducing end (Long, 1996). NodC (N-acetylglucosaminyltransferase) assemblies the backbone by the elongation of oligosaccharides (from two to six) at the non-reducing terminus (Cooper, 2007). Later, a deacetylase encoded by $\operatorname{nod} B$, removes the $N$-acetyl moiety from the non-reducing terminus of the $\mathrm{N}$-acetylglucosamine oligosaccharide; and finally NodA, an acyltransferase, links the acyl chain to the acetyl-free C-2 carbon of the non-reducing end of the oligosaccharide (Perret et al., 2000). Although nodABC genes are present in all symbiotic rhizobia, they are not functionally equivalent in all rhizobia species, since it has been demonstrated that NodA and NodC present host-specificity. NodA shows specificity for different fatty acid substrates, and NodC is determinant for the length of 
the backbone, and thus for host specificity (Roche et al., 1996). In certain cases, just the $\mathrm{N}$-acetyl-glucosaminyl oligosaccharide backbone is sufficient to trigger root hair deformation (Banfalvi and Kondorosi, 1989). Additional genes determine the specific decorations on NF core, such as fatty acids (nodEF), glycosylations (arabinosylation by noe $C$ and fucosylations by nodZ/nolK), sulfations (nodH/noeE), acetylations (nodL, nodX or nolL), $N$-methylation and carbamoylation (nodS, nodU and nolO) and 2-O methylation (noel). Not surprisingly, rhizobial mutants in nod genes are unable to nodulate legumes (Perret et al., 2000). However, even when so much information has been published on the influence of NF substitutions on host range, the host range cannot be predicted from NF structure alone.

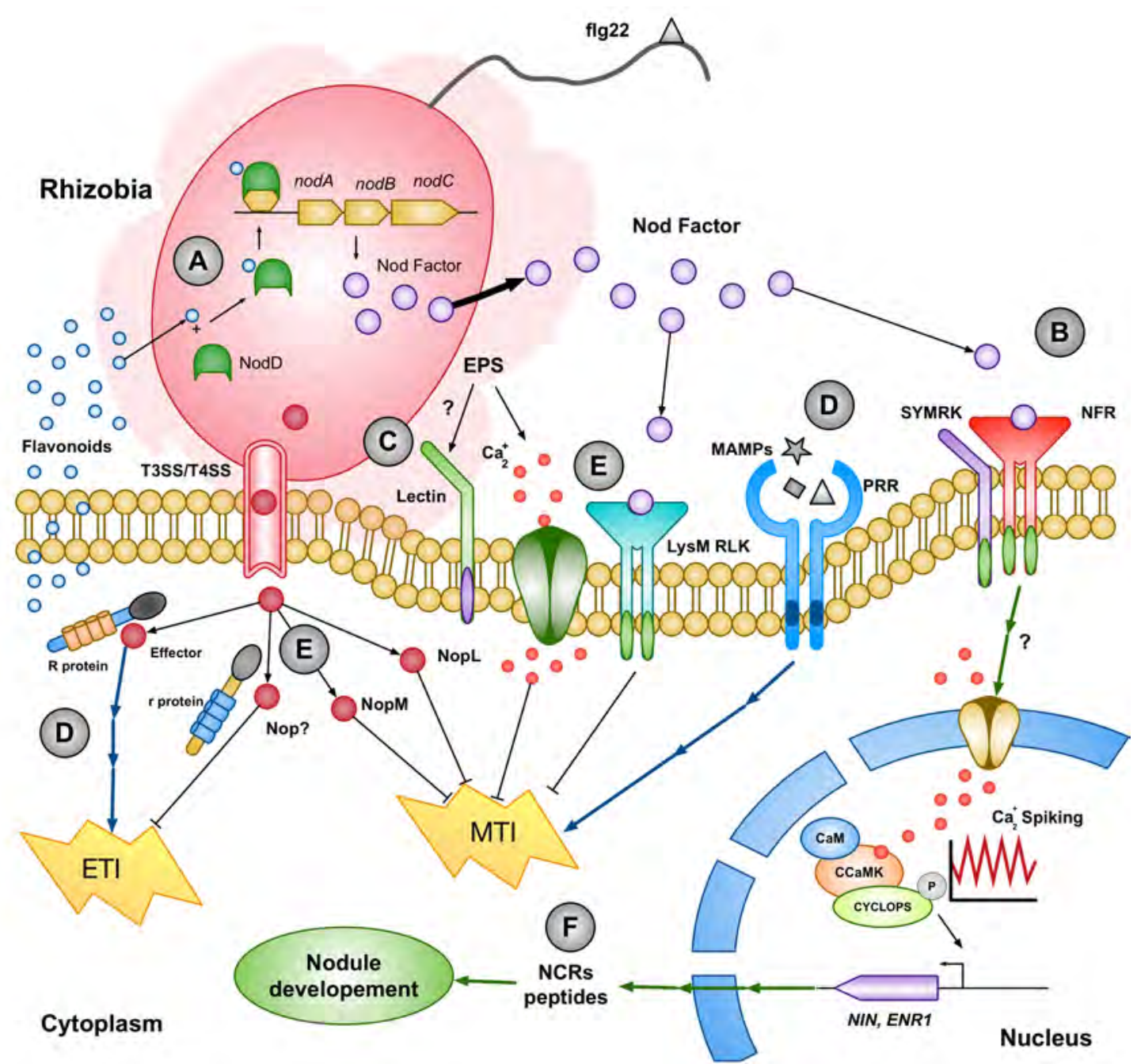

Figure I.3. Host Specificity and Symbiosis. A) Molecular dialogue between legume flavonoids and rhizobia NodD. B) interaction between NF (Nod Factor) and NFR (Nod Factor Receptor). C) Microbial surface polysaccharides helps to attach to the plant membrane. D) MAMPs Triggered Immunity (MTI) and Effector Triggered Immunity (ETI). E) MTI suppression by NF and EPS, and ETI suppression by NOPs. NCR peptides recognition and cleavage. Based on Broughton et al. (2003), Oldroyd (2013) and Gourion et al. (2015). 
Perception of NFs is mediated by NFRs, which are LysM domain-containing receptor-like kinases (Limpens et al., 2015) that bind directly to the NF (Broghammer et al., 2012). Due to the different NF structures, NFRs are a host determinant in symbiosis specificity (Wang et al., 2012). Two forms of NFRs, NFR1 and NFR5, have been described, and both are equally important for rhizobial colonization (Oldroyd, 2013). The interaction between NFRs and NF activates the symbiosis receptor-like kinases (SYMRK) and forms a complex, which stimulates calcium oscillations in the nuclear region and also by means of two $\mathrm{Ca}^{2+}$ channels located on the nuclear envelope. These $\mathrm{Ca}^{2+}$ ions interact with a calmodulin- and $\mathrm{Ca}$-dependent protein kinase (CCaMK) that phosphorylates the CYCLOPS protein. Afterwards, several transcriptional factors required for nodulation are induced, like nodule inception (NIN), which regulates NF-induced gene expression promoting nodule morphogenesis (Oldroyd et al., 2011, Oldroyd, 2013).

Distinct bacterial components that are involved in host specificity are the microbial surface polysaccharides, like exopolysaccharides (EPS), lipopolysaccharides (LPS), capsular polysaccharides (KPS) and cyclic ß-glucans. Alterations on surface polysaccharides result in symbiosis failures (Wang et al., 2012). EPSs are succinoglycan polymers and their alteration on the E. meliloti surface blocks infection thread formation and nodule development, even if these mutants induce hair curling (Finan et al., 1985, Leigh et al., 1985). A similar phenotype was found with ß-glucan exporting mutants (Dylan et al., 1986). Changes in EPS profile are correlated with host range in different systems, for example in the Medicago truncatula - E. meliloti system, strain NRG185 is able to form effective nodules in cultivar A17 but not in A20, while strain NRG247 has the opposite phenotype (Simsek et al., 2007). Similar phenotypes were found in other systems like with $B$. japonicum wild type/exoB mutant and Glycine soja/G. max (Parniske et al., 1994); or in Mesorhizobium loti EPS mutants, which are ineffective in Lotus leucocephala but effective in L. pedunculatus (Hotter and Scott, 1991). From the plant side, lectins have been described as probable EPS receptors. These molecules are carbohydrate-binding proteins that present a wide range of functions, from host defence to symbiosis (De Hoff et al., 2009). The lectin-EPS interaction has been shown to play a role in improving rhizobial attachment to hair roots (Figure I.3, C) (Laus et al., 2006).

During the initial phase of the symbiosis, defence responses may occur, but, generally, legumes tolerate rhizobia without eliciting them (Gourion et al., 2015). The plant immune system induces a number of walls against microbial attack. In the first phase, the recognition of microbial-associated molecular patterns (MAMPs) by host pattern recognition receptors (PRRs) triggers MAMP-triggered immunity (MTI) (Figure 
I.3, D). In phase two, successful pathogens deliver effectors that interfere with MTI, for example, using type III secretion systems (T3SS), resulting in effector-triggered susceptibility (ETS). The second layer of defence is the result of recognition of these effectors by plant resistance gene ( $R$ gene) products, which elicits effector-triggered immunity (ETI) (Figure I.3, D). Consequently, bacterial effectors and plant defence are host range determinants (Jones and Dangl, 2006). Plant defence is elicited by rhizobial inoculation (Lohar et al., 2006, Libault et al., 2010), but rhizobial MAMPs, like NFs or surface polysaccharides could play a role in the suppression of defence response in compatible legumes (Figure I.3, E). MTI reduces the fitness of nodulation; for instance, treatment with the active epitope of the bacterial flagellin, flg22, reduces the response to NF in L. japonicus (Lopez-Gomez et al., 2012). EPS has been proposed to suppress MTI by chelating $\mathrm{Ca}^{2+}$, an important cellular signal during MAMPs recognition (Aslam et al., 2008). Recently it was demonstrated that NFs partially suppress MTI, independently of NF recognition since this was verified in non-legume plants like Arabidopsis thaliana or Solanum lycopersicum (Liang et al., 2013). As mentioned before, some bacteria are able to suppress MTI by delivering effectors into plant cells, using secretion systems such as T3SS. Rhizobial strains also posses these secretion systems, and the induction of some of these T3SS is mediated by flavonoids (Wassem et al., 2008). T3SSs were found in some rhizobia like Bradyrhizobium diazoefficiens USDA110, M. loti MAFF303099, R. spNGR234 or E. fredii HH103 and USDA257. The effectors secreted by rhizobial T3SSs are known as nodulation outer proteins (NOPs), and are involved in host range. For instance, in $R$. sp. NGR234, mutations in T3SS could affect host range, either positively or negatively (Kambara et al., 2009). In addition to T3SSs, rhizobia also present type IV secretion systems (T4SSs), with functions similar to those of T3SSs (Figure I.3, E). By analogy with plant-pathogen interactions, legumes present $R$ genes that recognise rhizobial effectors. For example, depending on the of $R$ gene allele present in G. $\max$, the plant responds differently to different rhizobia. The presence of the Rj2 allele allow nodulation by $E$. fredii USDA257 but not by $B$. japonicum USDA122; however, the presence of the rj2 allele allows nodulation by both rhizobia (Yang et al., 2010).

During nodule development, bacteroids undergo a strictly controlled determination program, involving arrest in cell division, genomic endoreduplication, membrane permeabilization and size increase. Legumes that induce bacteroid differentiation, also encode for nodule-specific cysteine-rich (NCR) peptides, which are suggested to be responsible for these bacteroid modifications (Figure I.3, F). NCR peptides are defensin-type antimicrobial peptides, from thirty to sixty amino acids in length that share from four to six cysteine residues among them (Mergaert et al., 2006). In vitro, 
NCR peptides are able to induce bacteroid modification. In the $M$. truncatula $-E$. meliloti symbiosis, the loss of a plasmid results in a limitation in host range. This plasmid encodes a metallopeptidase responsible for NCR peptide cleavage in vitro (Crook et al., 2012, Price et al., 2015).

\subsection{Rhizobium leguminosarum}

Rhizobium leguminosarum $(\mathrm{RL})$ is a member of the $\alpha$-Proteobacteria that belongs to the order Rhizobiales. It was defined as new species in 1889 by Frank; it is the type strain of the genus Rhizobium, of the Rhizobiaceae family, and also of the order Rhizobiales. Its name stands for "which lives in a root of Leguminosae" (rhiza, root; bios in neutral form, life and leguminosarum, of Leguminosae) and it was accepted by The International Committee on Systematic Bacteriology as a proper species in 1980 (Sherman et al., 1980). Previous to that date, the Rhizobium species were defined by their symbiotic capabilities; however, in this genus, all the symbiosis-related genetic determinants are carried in a specific replicon, the symbiotic plasmid (pSym) and are, consequently, taxonomic untrustworthy (Young et al., 2001). Non-symbiotic isolates have also been found (Segovia et al., 1991, Laguerre et al., 1993a), and transfer of symbiotic plasmids to other Rhizobium genomic backgrounds also results in transfer of the symbiotic capabilities (Brewin et al., 1980, Abe et al., 1998).

Most studies on $\mathrm{RL}$ deal with its role in legume symbioses. However, this mutualistic relationship, visibly manifested in the form of root nodules, is not its exclusive lifestyle. The chances for any given symbiotic $R L$ cell in soil to find its corresponding host have been estimated as one in a million (Denison and Kiers, 2004), and $R L$ cells spend most of their viable life in the soil. $R L$ is a heterotrophic, obligate aerobic and micro-aerophilic microorganism that can utilize a wide range of carbon and nitrogen sources (Fuhrer et al., 2005); this catabolic versatility probably reflects its adaptation to survive -and thrive- in soil and in the rhizosphere of different host and non-host plants, and is probably responsible for its large and complex genome, close to the upper limit of bacterial genomes (Mitsui et al., 1997, Saito et al., 1998). RL possesses a strikingly large number of substrate transport, regulation and catabolic systems; for instance it has roughly 170 ATP-binding cassette (ABC) transport systems, which allows it to access a large diversity of low concentration soil nutrients (Boussau et al., 2004, Prell and Poole, 2006). 
$\mathrm{RL}$ has a multi-partite genome, in the form of one large, circular chromosome (4$5 \mathrm{Mb}$ ) and a variable number (1-6) of large or very large plasmids (Figure I.4) (Young et al., 2006, Reeve et al., 2010a, Reeve et al., 2010b, Reeve et al., 2015). Some of these resemble chromosomes in their size and functional complexity, and are known as "chromids" (Harrison et al., 2010). This particular genomic composition has been interpreted as a strategy to, at the same time, maintain a large amount of genetic information and reduce its generation time to 2-3h (Streit et al., 1991, Harrison et al., 2010, Jorrín and Imperial, 2015a).

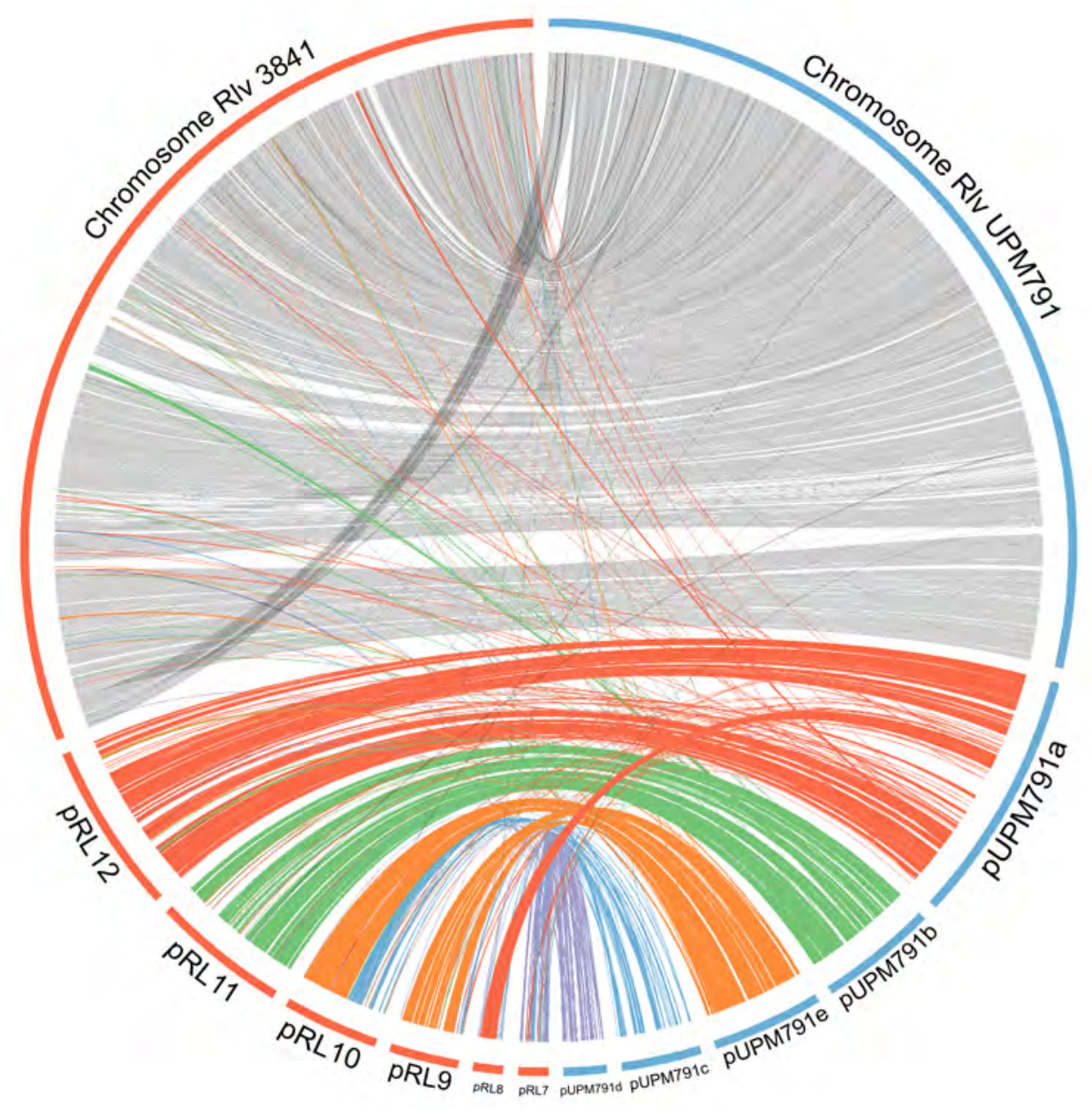

Figure I.4. Genome synteny between Rhizobium leguminosarum bv. viciae 3841 and $R$. leguminosarum bv. viciae UPM791. High conservation was shown between chromosome and chromids (pRL12 vs. pUPM791a and pRL11 vs. pRUPM791b) but not between the smallest plasmids. 
Traditionally, different RL biovars have been described depending on their host range. RL bv. trifolii (RIt) nodulates Trifolium spp., RL bv. viciae (RIv) establishes symbioses with Pisum spp., Lens spp., Vicia spp. and Lathyrus spp.; and RL bv. phaseoli (RIp) forms nodules with Phaseolus spp. (Hynes and Oconnell, 1990, Laguerre et al., 1993a, Doyle and Luckow, 2003). It is noteworthy that Rlv can

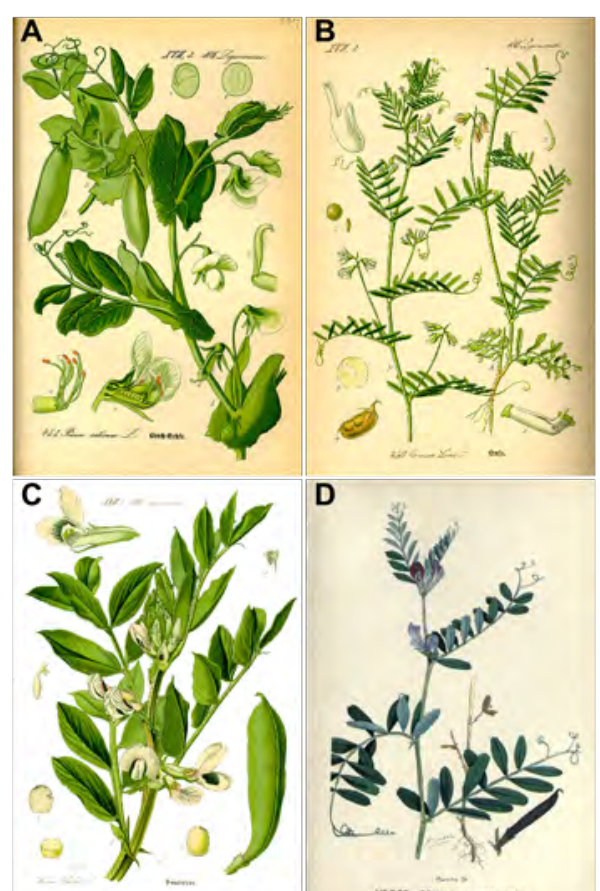

Figure I.5. Rhizobium leguminosarum bv. viciae legume hosts. A (Pisum sativum), B (Lens culinaris), C (Vicia faba) and D (V. sativa). establish symbioses with different genera within the Fabeae tribe that exhibit adaptations to different edaphoclimatic conditions. It has been proposed that some Rlv genotypes could be more adapted to specific plant genera or genotypes (Davis et al., 1988, Bromfield et al., 1995, Hartmann et al., 1998, Zeze et al., 2001, Laguerre et al., 2003). This would imply that the plant selects for specific rhizobial genotypes from those present in soil (Jorrín and Imperial, 2015b). Evidence for this plant-selection hypothesis has been reported in the past by using different strain typing methods. These early studies showed differences in RIv genotype preference between pea and fava bean plants, with fava bean plants exhibiting high specificity for certain rhizobial nod genotypes, independently of their chromosomal background. Pea plants, however, selected RIv strains where chromosomal and symbiotic genotypes correlated (Hynes and Oconnell, 1990, Evans et al., 1996, Laguerre et al., 2003, Mutch and Young, 2004). Another conclusion from these studies was that neither pea nor fava bean plants exploited the total RIv diversity present in soil. Regarding other hosts, such as lentil and vetch, little information is available, except for the fact that lentil plants show a higher number of different chromosomal backgrounds than pea, and that vetch, like pea, shows less specificity than fava bean (Brockman and Bezdicek, 1989, Hynes and Oconnell, 1990, Laguerre et al., 1993b, Evans et al., 1996, Laguerre et al., 2003, Mutch and Young, 2004). These studies relied on plasmid profiles or on the use of polymerase chain reaction-amplified, restriction fragment length polymorphism analysis (PCR-RFLP) of symbiotic and nonsymbiotic molecular markers, and offered little information on the nature or on the basis of this selection. 
2. OBJECTIVES 

The aim of this Thesis was to characterize the genomic, genetic and molecular bases of the selection of specific Rhizobium leguminosarum bv. viciae genotypes by different host legumes (Pisum sativum, Lens culinaris, Vicia faba and V. sativa) from a well-known agricultural soil.

This general aim was fulfilled through the following specific objectives:

1. Characterization of the rhizobial population genomics of the Rhizobium leguminosarum-legume hosts symbiosis.

1.1. Design of a bioinformatics pipeline to study rhizobial population genomics.

1.2. Genomic characterization of the Rhizobium leguminosarum subpopulations (PoolSeq) selected by each legume host.

2. Characterization of the native Rhizobium leguminosarum soil population and comparison with plant host-selected subpopulations.

2.1. Genomic characterization of the native Rhizobium leguminosarum soil population.

2.2. Comparison of the native soil Rhizobium leguminosarum with the host-selected Rhizobium leguminosarum subpopulations.

3. Characterization of mesocosm host-selected Rhizobium leguminosarum subpopulations and comparison with soil and plant-host selected subpopulations.

3.1. Elucidation of the population genomic bases for differential plant-host preferences for specific genotypes.

3.2. Genomic analysis, at the strain level, of the host preferred genotypes after mesocosm selection. 

3. MATERIALS AND METHODS 



\subsection{SITE AND SOIL CHARACTERISTICS}

The source of the soil rhizobial population used for all the experiments in this Thesis was P1 soil, collected in the Institut National de la Recherche Agronomique (INRA) Experimental Station of Époisses (Domain experimental d'Époisses, Bretenière, Côte d'Or, France), and it was made available to us by Dr. Gisèle Laguerre in September 2010. For soil collection, the upper crust $(3-5 \mathrm{~cm})$ from several sites within the P1 plot was removed, and portions of soil down to $20-30 \mathrm{~cm}$ depth were collected with a shovel. All portions were mixed thoroughly and $100 \mathrm{~kg}$ were shipped to our Centre, where it was stored at room temperature. Before use, all soil portions were sieved with a $5 \mathrm{~mm}$ pore-diameter sieve. The analytical characteristics of $\mathrm{P} 1$ soil were published by Louvrier et al. (1996): $\mathrm{pH} 8,36 \%$ clay, $50 \%$ silt, $14 \%$ sand, $3.6 \%$ organic matter. The number of symbiotic $R$. leguminosarum bv. viciae (RIv) cells was estimated to be $10^{4}-10^{5}$ per gram of soil by an indirect plant infection method (most probable number, MPN) with pea plants as trap host (Louvrier et al., 1996), and $10^{4}$ per gram by a direct isolation method (Louvrier et al., 1995). As far as recorded, no rhizobial inoculations had taken place in the area. However, peas were last planted in the P1 plot in 1982. From that date, only non-legume crops were planted, mainly corn and wheat, up until sampling (Laguerre et al., 2003) (G. Laguerre personal communication).

Samples of soil taken after five cycles of host-selection $\left(\right.$ pea $_{A}$, pea $_{B}$, lentil ${ }_{A}$, lentil ${ }_{B}$, fava bean $n_{A}$, fava bean $n_{B}$, vetch ${ }_{A}$ and vetch $_{B}$ ), as well as the original soil (bulk), were externally analyzed (Laboratorio de Edafología y Técnicas Analíticas Instrumentales, EUIT Agrícola, UPM, Madrid) for physico-chemical characteristics (Table M.1).

Table M.1 Soil chemical analysis for each soil previous (Bulk) and after five cycles of hostselection

\begin{tabular}{lccccccccccc}
\hline & $\mathrm{pH}$ & $\mathrm{SAL}$ & $\mathrm{OM}$ & $\mathrm{T}-\mathrm{N}_{2}$ & $\mathrm{C} / \mathrm{N}$ & $\mathrm{A} . \mathrm{P}$. & $\mathrm{CaCO}_{3}$ & $\mathrm{Na}$ & $\mathrm{K}$ & $\mathrm{Ca}$ & $\mathrm{Mg}$ \\
\hline Bulk & 7.6 & 140 & 2.51 & 0.204 & 7.2 & 129.6 & 10.3 & 66 & 1,603 & 7,080 & 163 \\
\hline Pea $_{\mathrm{A}}$ & 7.8 & 155 & 2.3 & 0.187 & 7.2 & 120.6 & 9.7 & 358 & 1,210 & 6,505 & 539 \\
Pea $_{\mathrm{B}}$ & 7.9 & 270 & 2.28 & 0.187 & 7.1 & 124 & 8.2 & 162 & 1,023 & 6,311 & 535 \\
Lentil $_{\mathrm{A}}$ & 7.8 & 360 & 2.35 & 0.201 & 6.8 & 123.4 & 8.5 & 188 & 1,096 & 5,596 & 535 \\
Lentil $_{\mathrm{B}}$ & 7.8 & 417 & 2.25 & 0.179 & 7.3 & 124.2 & 10 & 181 & 1,092 & 6,525 & 503 \\
Fava bean $_{\mathrm{A}}$ & 7.7 & 392 & 2.17 & 0.192 & 6.6 & 132.6 & 9.7 & 198 & 1,032 & 6,690 & 464 \\
Fava bean $_{\mathrm{B}}$ & 7.8 & 351 & 2.27 & 0.193 & 6.8 & 132.6 & 9 & 203 & 1,133 & 6,388 & 483 \\
Vetch $_{\mathrm{A}}$ & 8.0 & 250 & 2.31 & 0.187 & 7.2 & 115.2 & 8.4 & 209 & 1,161 & 6,186 & 508 \\
Vetch $_{\mathrm{B}}$ & 7.8 & 278 & 2.22 & 0.186 & 6.9 & 116.4 & 8.9 & 170 & 1,091 & 6,188 & 572 \\
\hline
\end{tabular}

SAL (salinity, $\mu \mathrm{S} / \mathrm{cm}$ ), OM (soil \% of organic material by oxidation), $\mathrm{T}-\mathrm{N}_{2}$ (\% Total Nitrogen), C/N (carbon/nitrogen ratio), A. P. (assimilable phosphate) in ppm (parts per million), $\mathrm{CaCO}_{3}(\%$ calcium carbonate); Cation exchange for $\mathrm{Na}$ (sodium), $\mathrm{K}$ (potassium), $\mathrm{Ca}$ (calcium) and $\mathrm{Mg}$ (magnesium) in ppm. 


\subsection{PLANT MATERIAL}

Commercial seeds from pea (Pisum sativum L. cv. Frisson), lentil (Lens culinaris Medik. Cv. Magda), fava bean (Vicia faba L. cv. Muchamiel), vetch (V. sativa L. cv. Senda), white clover (Trifolium repens L. cv. Huia) and bean (Phaseolus vulgaris L. cv. nanus Alba) were used in the present Thesis. All the seeds were stored at $4^{\circ} \mathrm{C}$.

\subsection{BACTERIAL STRAINS}

Rhizobium leguminosarum bv. viciae 3841 , a strain isolated from a pea nodule (Johnston and Beringer, 1975) was the first Rlv strain whose genome was sequenced (Young et al., 2006) and has been used as reference throughout this work. $R$. leguminosarum bv. viciae UPM791, also a typical pea isolate, has been thoroughly used as inoculant (Nitragin strain 128C53, (Ruiz-Argüeso et al., 1983)) and studied for years by our group in view of its hydrogen recycling capabilities (Leyva et al., 1987, Brito et al., 1994, Gutierrez et al., 1997, Baginsky et al., 2002, Albareda et al., 2012). Other rhizobial strains were isolated from P1 soil, either from nodules, using plant hosts as traps (Tables M.2 and M.4), or directly from the soil (Table M.3).

Table M.2. Endosymbiotic bacteria obtained in Chapter $1(1 / 2)$

\begin{tabular}{|c|c|c|c|c|c|c|c|c|c|}
\hline Legume host & \multicolumn{9}{|c|}{ strains } \\
\hline \multirow{12}{*}{ Pisum sativum } & G001 & G002 & G003 & G004 & G005 & G006 & G007 & G008 & G009 \\
\hline & G010 & G011 & G012 & G013 & G014 & G015 & G016 & G017 & G018 \\
\hline & G019 & G020 & G021 & G022 & G023 & G024 & G027 & G028 & G029 \\
\hline & G030 & G031 & G032 & G035 & G036 & G037 & G038 & G039 & G040 \\
\hline & G041 & G042 & G043 & G045 & G046 & G047 & G049 & G050 & G051 \\
\hline & G052 & G053 & G054 & G055 & G056 & G057 & G058 & G059 & G060 \\
\hline & G061 & G062 & G063 & G064 & G065 & G066 & G067 & G069 & G070 \\
\hline & G071 & G072 & G073 & G074 & G077 & G078 & G079 & G080 & G081 \\
\hline & G082 & G083 & G084 & G085 & G086 & G087 & G088 & G089 & G090 \\
\hline & G091 & G092 & G093 & G094 & G095 & G096 & G097 & G098 & G099 \\
\hline & G100 & G101 & G102 & G103 & G104 & G105 & G106 & G107 & G108 \\
\hline & G109 & & & & & & & & \\
\hline \multirow{12}{*}{ Lens culinaris } & L001 & L002 & L003 & L004 & L005 & L006 & L007 & L008 & L009 \\
\hline & L010 & L011 & L012 & L013 & L014 & L015 & L016 & L017 & L018 \\
\hline & L019 & L020 & L025 & L026 & L027 & L028 & L029 & L030 & L035 \\
\hline & L036 & L041 & L042 & L044 & L045 & L046 & L047 & L048 & L049 \\
\hline & L050 & L051 & L053 & L054 & L056 & L057 & L058 & L059 & L060 \\
\hline & L061 & L062 & L063 & L065 & L066 & L067 & L068 & L069 & L070 \\
\hline & L071 & L072 & L073 & L074 & L075 & L076 & L077 & L078 & L079 \\
\hline & L080 & L081 & L082 & L084 & L085 & L086 & L087 & L088 & L089 \\
\hline & L090 & L091 & L092 & L093 & L094 & L095 & L096 & L098 & L099 \\
\hline & L100 & L101 & L102 & L103 & L104 & L106 & L108 & L109 & L110 \\
\hline & L111 & L112 & L113 & L115 & L116 & L117 & L118 & L119 & L120 \\
\hline & L121 & & & & & & & & \\
\hline
\end{tabular}


Table M.2. Endosymbiotic bacteria obtained in Chapter 1 (2/2)

\begin{tabular}{|c|c|c|c|c|c|c|c|c|c|}
\hline Legume host & \multicolumn{9}{|c|}{ strains } \\
\hline \multirow{12}{*}{ Vicia faba } & H001 & $\mathrm{H} 002$ & $\mathrm{H} 003$ & $\mathrm{H} 005$ & H006 & $\mathrm{H} 008$ & $\mathrm{H} 009$ & $\mathrm{H} 010$ & H011 \\
\hline & H012 & H013 & H015 & H016 & H017 & $\mathrm{H} 024$ & $\mathrm{H} 026$ & H027 & $\mathrm{H} 028$ \\
\hline & $\mathrm{H} 029$ & H030 & H031 & $\mathrm{H} 033$ & $\mathrm{H} 034$ & H035 & H036 & H038 & H039 \\
\hline & $\mathrm{H} 043$ & H044 & H047 & $\mathrm{H} 049$ & $\mathrm{H} 050$ & H052 & $\mathrm{H} 055$ & H056 & H059 \\
\hline & $\mathrm{H} 060$ & H071 & H072 & $\mathrm{H} 077$ & $\mathrm{H} 078$ & H082 & H091 & $\mathrm{H} 093$ & H094 \\
\hline & H098 & H099 & $\mathrm{H} 100$ & H109 & $\mathrm{H} 110$ & $\mathrm{H} 111$ & H115 & $\mathrm{H} 117$ & H123 \\
\hline & H125 & H126 & $\mathrm{H} 127$ & $\mathrm{H} 128$ & $\mathrm{H} 129$ & $\mathrm{H} 130$ & $\mathrm{H} 132$ & H133 & $\mathrm{H} 134$ \\
\hline & H135 & H136 & H138 & $\mathrm{H} 139$ & $\mathrm{H} 142$ & $\mathrm{H} 145$ & $\mathrm{H} 148$ & H149 & H152 \\
\hline & H153 & H154 & H155 & H156 & $\mathrm{H} 158$ & $\mathrm{H} 159$ & $\mathrm{H} 160$ & H161 & H162 \\
\hline & H163 & H164 & H166 & H167 & $\mathrm{H} 168$ & H169 & $\mathrm{H} 170$ & H171 & $\mathrm{H} 174$ \\
\hline & H175 & $\mathrm{H} 177$ & $\mathrm{H} 178$ & H179 & $\mathrm{H} 180$ & $\mathrm{H} 181$ & H188 & H189 & $\mathrm{H} 190$ \\
\hline & H192 & & & & & & & & \\
\hline \multirow{11}{*}{ Vicia sativa } & V001 & V002 & V003 & V004 & V005 & V006 & V007 & V008 & V009 \\
\hline & V010 & V011 & V012 & V013 & V014 & V015 & V016 & V017 & V018 \\
\hline & V019 & V020 & V026 & V030 & V031 & V041 & V042 & V043 & V044 \\
\hline & V045 & V046 & V047 & V048 & V049 & V050 & V051 & V054 & V055 \\
\hline & V056 & V057 & V058 & V059 & V060 & V061 & V062 & V065 & V066 \\
\hline & V067 & V068 & V069 & V070 & V071 & V072 & V073 & V074 & V075 \\
\hline & V076 & V077 & V078 & V079 & V080 & V081 & V082 & V083 & V084 \\
\hline & V085 & V086 & V088 & V089 & V090 & V091 & V092 & V093 & V094 \\
\hline & V095 & V096 & V097 & V098 & V099 & V100 & V101 & V102 & V103 \\
\hline & V104 & V105 & V106 & V109 & V110 & V111 & V112 & V113 & V114 \\
\hline & V115 & V116 & V117 & V118 & V119 & V120 & & & \\
\hline
\end{tabular}

Table M.3. Bacteria obtained in Chapter 2

\begin{tabular}{|c|c|c|c|c|c|c|c|c|}
\hline Source & \multicolumn{8}{|c|}{ Strain } \\
\hline \multirow{15}{*}{ P1 soil } & S01A03 & S01A39 & S03A12 & S03A13 & S03A20 & S03A21 & S03A23 & S03A24 \\
\hline & S03A30 & S03A33 & S03B01 & S03B04 & S03B08 & S03B10 & S03B17 & S03B19 \\
\hline & S03B20 & S03B21 & S03B25 & S03B27 & S03B32 & S03B44 & S03B46 & S03B47 \\
\hline & S03B49 & S03B53 & S03C09 & S03D22 & S03D26 & S04010 & S04022 & S04023 \\
\hline & S04043 & S04054 & S04085 & S05024 & S05036 & S05037 & S05050 & S05054 \\
\hline & S05055 & S05056 & S05061 & S05071 & S06027 & S06029 & S06034 & S06040 \\
\hline & S06057 & S06065 & S06136 & S06154 & S06190 & S06190 & S06200 & S07001 \\
\hline & S07007 & S07044 & S07051 & S07109 & S08013 & S08022 & S08044 & S08069 \\
\hline & S09003 & S09012 & S09013 & S09014 & S09020 & S09031 & S09037 & S09039 \\
\hline & S09067 & S09077 & S09109 & S09112 & S09113 & S09123 & S09130 & S09133 \\
\hline & S10001 & S10022 & S10024 & S10036 & S10038 & S10040 & S10054 & S10058 \\
\hline & S10103 & S10156 & S10157 & S10158 & S10165 & S11009 & S11017 & S11025 \\
\hline & S11031 & S11064 & S11070 & S11078 & S11101 & S11115 & S11184 & S11185 \\
\hline & S11190 & S11195 & S11200 & S12024 & S12067 & S12076 & S12092 & S12097 \\
\hline & & 031 & & & S13064 & 13064 & & \\
\hline
\end{tabular}


Table M.4. Endosymbiotic bacteria obtained in Chapter 3

\begin{tabular}{|c|c|c|c|c|c|c|}
\hline \multicolumn{2}{|c|}{ source } & \multicolumn{5}{|c|}{ Strains } \\
\hline \multirow{10}{*}{ Pisum sativum } & \multirow{5}{*}{ pea $_{A}$} & GPS1_01 & GPS1_02 & GPS1_03 & GPS1_04 & GPS1_05 \\
\hline & & GPS1_06 & GPS1_07 & GPS1_08 & GPS1_09 & GPS1_10 \\
\hline & & GPS1_11 & GPS1_12 & GPS1_13 & GPS1_14 & GPS1_15 \\
\hline & & GPS1_16 & GPS1_17 & GPS1_18 & GPS1_19 & GPS1_20 \\
\hline & & GPS1_21 & GPS1_22 & GPS1_23 & GPS1_24 & GPS1_25 \\
\hline & \multirow{5}{*}{ pea $_{B}$} & GPS2_01 & GPS2_02 & GPS2_03 & GPS2_04 & GPS2_05 \\
\hline & & GPS2_06 & GPS2_07 & GPS2_08 & GPS2_09 & GPS2_10 \\
\hline & & GPS2_11 & GPS2_12 & GPS2_13 & GPS2_14 & GPS2_15 \\
\hline & & GPS2_16 & GPS2_17 & GPS2_18 & GPS2_19 & GPS2_20 \\
\hline & & GPS2 21 & GPS2 22 & GPS2 23 & GPS2 24 & GPS2 25 \\
\hline \multirow{10}{*}{ Lens culinaris } & \multirow{5}{*}{ lentil $_{A}$} & LPS1_02 & LPS1_03 & LPS1_05 & LPS1_06 & LPS1_10 \\
\hline & & LPS1_11 & LPS1_13 & LPS1_15 & LPS1_16 & LPS1_17 \\
\hline & & LPS1_18 & LPS1_19 & LPS1_21 & LPS1_22 & LPS1_23 \\
\hline & & LPS1_24 & LPS1_25 & LPS1_26 & LPS1_27 & LPS1_28 \\
\hline & & LPS1_29 & LPS1_30 & LPS1_31 & LPS1_34 & LPS1_35 \\
\hline & \multirow{5}{*}{ lentil $_{B}$} & LPS2_01 & LPS2_02 & LPS2_03 & LPS2_04 & LPS2_05 \\
\hline & & LPS2_06 & LPS2_07 & LPS2_08 & LPS2_09 & LPS2_10 \\
\hline & & LPS2_11 & LPS2_12 & LPS2_13 & LPS2_14 & LPS2_15 \\
\hline & & LPS2_16 & LPS2_17 & LPS2_18 & LPS2_19 & LPS2_20 \\
\hline & & LPS2_21 & LPS2_22 & LPS2_23 & LPS2_24 & LPS2_25 \\
\hline \multirow{10}{*}{ Vicia faba } & \multirow{5}{*}{ fava bean be $_{A}$} & HPS1_01 & HPS1_02 & HPS1_03 & HPS1_04 & HPS1_05 \\
\hline & & HPS1_06 & HPS1_07 & HPS1_09 & HPS1_10 & HPS1_11 \\
\hline & & HPS1_12 & HPS1_13 & HPS1_14 & HPS1_15 & HPS1_16 \\
\hline & & HPS1_17 & HPS1_18 & HPS1_21 & HPS1_22 & HPS1_23 \\
\hline & & HPS1_25 & HPS1_26 & HPS1_27 & HPS1_28 & HPS1_31 \\
\hline & \multirow{5}{*}{ fava bean $_{B}$} & HPS2_01 & HPS2_03 & HPS2_04 & HPS2_05 & HPS2_06 \\
\hline & & HPS2_07 & HPS2_08 & HPS2_10 & HPS2_11 & HPS2_12 \\
\hline & & HPS2_13 & HPS2_15 & HPS2_16 & HPS2_17 & HPS2_18 \\
\hline & & HPS2_19 & HPS2_20 & HPS2_21 & HPS2_22 & HPS2_23 \\
\hline & & HPS2_24 & HPS2_25 & HPS2_26 & HPS2_27 & HPS2_29 \\
\hline \multirow{10}{*}{ Vicia sativa } & \multirow{5}{*}{ vetch $_{A}$} & VPS1_01 & VPS1_02 & VPS1_03 & VPS1_04 & VPS1_05 \\
\hline & & VPS1_06 & VPS1_07 & VPS1_09 & VPS1_11 & VPS1_12 \\
\hline & & VPS1_13 & VPS1_14 & VPS1_15 & VPS1_16 & VPS1_17 \\
\hline & & VPS1_18 & VPS1_19 & VPS1_21 & VPS1_23 & VPS1_24 \\
\hline & & VPS1_25 & VPS1_26 & VPS1_27 & VPS1_28 & VPS1_31 \\
\hline & \multirow{5}{*}{ vetch $_{B}$} & VPS2_01 & VPS2_02 & VPS2_03 & VPS2_04 & VPS2_05 \\
\hline & & VPS2_06 & VPS2_08 & VPS2_09 & VPS2_10 & VPS2_11 \\
\hline & & VPS2_12 & VPS2_13 & VPS2_14 & VPS2_15 & VPS2_16 \\
\hline & & VPS2_17 & VPS2_18 & VPS2_20 & VPS2_21 & VPS2_22 \\
\hline & & VPS2_23 & VPS2_24 & VPS2_25 & VPS2_26 & VPS2_27 \\
\hline
\end{tabular}




\subsection{CULTURE MEDIA AND GROWTH CONDITIONS}

All the bacterial cultures were grown at $28^{\circ} \mathrm{C}$. Liquid cultures were grown in a rotatory shaker at 200 revolutions per minute (rpm). Solid media used for plating contained $1.5 \%$ of agar unless otherwise specified.

The following media were used in this study:

Yeast mannitol (YM): mannitol $1 \mathrm{~g} \cdot \mathrm{L}^{-1}$, yeast extract $0.4 \mathrm{~g} \cdot \mathrm{L}^{-1}, \mathrm{NaCl} 0.1 \mathrm{~g} \cdot \mathrm{L}^{-1}, \mathrm{KH}_{2} \mathrm{PO}_{4}$ $0.1 \mathrm{~g} \cdot \mathrm{L}^{-1}, \mathrm{MgSO}_{4} 0.2 \mathrm{~g} \mathrm{~g} \cdot \mathrm{L}^{-1} ; \mathrm{pH} 6.8$ (Vincent, 1970).

Tryptone yeast extract (TY): tryptone $6 \mathrm{~g} \cdot \mathrm{L}^{-1}$, yeast extract $3 \mathrm{~g} \cdot \mathrm{L}^{-1}, \mathrm{CaCl}_{2} 0.5 \mathrm{~g} \cdot \mathrm{L}^{-1}$ (Beringer, 1974).

Luria-Bertani broth (LB): tryptone $10 \mathrm{~g} \cdot \mathrm{L}^{-1}$, yeast extract $5 \mathrm{~g} \cdot \mathrm{L}^{-1} \mathrm{NaCl} 5 \mathrm{~g} \cdot \mathrm{L}^{-1}$ (Sambrook and Russell, 2001).

Trypticasein Soy broth (TS): $30 \mathrm{~g} \cdot \mathrm{L}^{-1}$. (Conda, Madrid, Spain)

MNBP: mannitol g. $\mathrm{L}^{-1}, \mathrm{Na}_{2} \mathrm{HPO}_{4} 178 \mathrm{mg} \cdot \mathrm{L}^{-1}, \mathrm{MgSO}_{4} 100 \mathrm{mg} \cdot \mathrm{L}^{-1}, \mathrm{FeCl}_{3} 33.25 \mathrm{mg} \cdot \mathrm{L}^{-1}$, $\mathrm{CaCl}_{2} 53 \mathrm{mg} \cdot \mathrm{L}^{-1}, \mathrm{NH}_{4} \mathrm{NO}_{3} 500 \mathrm{mg} \cdot \mathrm{L}^{-1}$, thiamine $500 \mu \mathrm{g} \cdot \mathrm{L}^{-1}$, biotin $500 \mu \mathrm{g} \cdot \mathrm{L}^{-1}, \mathrm{Ca}$ pantothenate $600 \mu \mathrm{g} \cdot \mathrm{L}^{-1}$, cycloheximide $100 \mathrm{mg} \cdot \mathrm{L}^{-1}$, bacitracin $25 \mathrm{mg} \cdot \mathrm{L}^{-1}$, penicillin $\mathrm{G} 3$ $\mathrm{mg} \cdot \mathrm{L}^{-1}$, benomyl $5 \mathrm{mg} \cdot \mathrm{L}^{-1}$, pentachloronitrobenzene $3.5 \mathrm{mg} \cdot \mathrm{L}^{-1}$; Congo red $25 \mathrm{ppm} ; \mathrm{pH}$ 6.8 (Louvrier et al., 1995).

Antibiotics used in this work have been obtained from Sigma-Aldrich Co (St. Louis, Missouri, USA). Stock solutions were prepared and stored as described by Sambrook and Russell (2001).

\subsection{OLIGONUCLEOTIDES}

For specific oligonucleotide primer design, sequences for the fnrN, glnIl, nodC and nifH genes from strains RIv 3841, RIt WSM1325 and RIt WSM2304 were obtained from the Genbank database (http://www.ncbi.nlm.nih.gov/genbank/). Sequences for each gene were aligned with ClustalW2 (http://www.ebi.ac.uk/Tools/msa/clustalw2/), and primers were designed in conserved regions. Primers for $r p o B$ were designed in the first hipervariable region (Khamis et al., 2003), using an alignment of different Rhizobium spp. rроB genes from the Genbank database. Primers were designed from multiple alignments either using the PrimerBlast online tool (http://www.ncbi.nlm.nih.gov/tools/primer-blast/) or manually, and ordered from Sigma Aldrich Spain (Madrid). Stocks were stored at $100 \mu \mathrm{M}$ in water at $-20^{\circ} \mathrm{C}$. All primers used in this Thesis are listed in Table M5 below. 
Table M.5. List of primers used in this Thesis

\begin{tabular}{|c|c|c|c|}
\hline Name & Sequence $\left(5^{\prime}-3^{\prime}\right)$ & Product & \\
\hline F_Rleg_FnrN & GGTGATGCCGGACGGGCG & \multirow{2}{*}{$f n r N$} & \multirow{2}{*}{ This Thesis } \\
\hline R_Rleg_FnrN & TGGAGCAGGCTTGCGACCTT & & \\
\hline F_Rleg_glnll & GCCGCAGCTCCGATTGCGTG & \multirow{2}{*}{$g \mid n l l$} & \multirow{2}{*}{ This Thesis } \\
\hline R_Rleg_glnll & AATCTGGATTCCCACTGGCCCTTG & & \\
\hline F_Rleg_NodC_DEG & TTGCAGGCMAGCCAGTACTCC & \multirow[b]{2}{*}{ nodC } & \multirow[b]{2}{*}{ This Thesis } \\
\hline R_Rleg_NodC & GGAAAGCGCAAAGCGCAAATCG & & \\
\hline F_Rleg_NifH_DEG & GCWGCTCTGCGTCAGATCGC & \multirow[b]{2}{*}{ nifH } & \multirow[b]{2}{*}{ This Thesis } \\
\hline R_Rleg_NifH_DEG & TGGACAATGTTRTCGCGYGGC & & \\
\hline F_rpoB_583 & GARTTCGACGCCAAGGAYAT & \multirow[b]{2}{*}{$r p o B$} & \multirow[b]{2}{*}{ This Thesis } \\
\hline R_rpoB_1200 & GAAGAACAGCGAGTTGAACAT & & \\
\hline nodC_F_540 & TGATYGAYATGGARTAYTGGCT & \multirow{2}{*}{$\operatorname{nod} C$} & \multirow{2}{*}{ Sarita et al 2005} \\
\hline nodC_R_1160 & CGYGACARCCARTCGCTRTTG & & \\
\hline
\end{tabular}




\subsection{PLANT EXPERIMENTS}

\subsubsection{Seed sterilization and germination}

For all the plant experiments seedlings were obtained from surface-sterilized seeds and different protocols were used for different species. Peas and vetches were sterilized with $70 \%$ ethanol, $1 \mathrm{~min}$, followed by bleach diluted 1:4, $3 \mathrm{~min}$; lentils were treated with bleach diluted 1:20, 5 min; fava beans were decontaminated with bleach at $1: 4$ and $0.1 \%$ Tween 20 , shaking at $200 \mathrm{rpm}$ during $20 \mathrm{~min}$ at $28^{\circ} \mathrm{C}$; clovers were sterilized with $70 \%$ ethanol, $15 \mathrm{~min}$, and 1:10 bleach, $15 \mathrm{~min}$; and beans were disinfected 5 min with bleach. Bleach was removed by serial washes with sterile water; afterwards seeds were stratified overnight at $4^{\circ} \mathrm{C}$; except for fava beans that were embedded in water overnight at $28^{\circ} \mathrm{C}$ with $200 \mathrm{rpm}$ shaking. All seeds were germinated in $1 \%$ agar plates at $22^{\circ} \mathrm{C}$ in darkness for 3 days.

\subsubsection{Trap plant assay}

P. sativum, L. culinaris, V. faba and V. sativa were used as trap plants. The experiment was carried out in Leonard jars with three plants per jar and four jars per plant type. Each jar was filled with a mixture (1:1) of P1 soil and sterile vermiculite. Plants were cultivated for three weeks in Leonard jars containing Jensen's solution $\left(\mathrm{KCl} 74.5 \mathrm{mg} \cdot \mathrm{L}^{-1}, \mathrm{~K}_{2} \mathrm{HPO}_{4} 174 \mathrm{mg} \cdot \mathrm{L}^{-1}, \mathrm{MgSO}_{4} \cdot 7 \mathrm{H}_{2} \mathrm{O} 246.5 \mathrm{mg} \cdot \mathrm{L}^{-1}\right.$, ferric citrate 1.8 $\mathrm{mg} \cdot \mathrm{L}^{-1}, \mathrm{CaSO}_{4} 344 \mathrm{mg} \mathrm{L}-1, \mathrm{CuSO}_{4} \cdot 5 \mathrm{H}_{2} \mathrm{O} 39 \mu \mathrm{g} \cdot \mathrm{L}^{-1}, \mathrm{ZnSO}_{4} \cdot 5 \mathrm{H}_{2} \mathrm{O} 110 \mu \mathrm{g} \cdot \mathrm{L}^{-1}$, $\mathrm{MnSO}_{4} \cdot 7 \mathrm{H}_{2} \mathrm{O} \quad 1.015 \mathrm{mg} \cdot \mathrm{L}^{-1}, \quad\left(\mathrm{NH}_{4}\right)_{6} \mathrm{Mo}_{7} \mathrm{O}_{24} \cdot 4 \mathrm{H}_{2} \mathrm{O} \quad 5 \quad \mu \mathrm{g} \cdot \mathrm{L}^{-1}, \mathrm{H}_{3} \mathrm{BO}_{3} 715 \mu \mathrm{g} \cdot \mathrm{L}^{-1}$; (Somasegaran, 1994)) Plants grown on vermiculite without any soil addition were used as negative nodulation controls.

\subsubsection{Isolation of Rhizobium strains from nodules}

Endosymbiotic bacteria were isolated from nodules induced in $P$. sativum, $L$. culinaris, V. faba and $V$. sativa trap plants by bacteria present in soil samples. After three weeks, red nodules were picked from plants and were surface-sterilized (1 min $70 \%$ ethanol, followed by $3 \mathrm{~min} 1: 4$ bleach) and crushed aseptically. Bacteria were isolated and purified in YM agar plates following standard techniques (Vincent, 1970). The strains were grown in $\mathrm{YM}$ at $28^{\circ} \mathrm{C}$ and maintained at $-80^{\circ} \mathrm{C}$ in $20 \%$ of glycerol for long-term storage. 


\subsubsection{Inoculation assay}

In order to test the symbiotic capabilities of strains obtained in Chapter 1, plant experiments were carried out in Leonard jars containing Jensen's solution with three surface-sterilized seedlings per jar. Seedlings were inoculated with $1 \mathrm{ml}$ of early stationary phase Rlv cultures. Plants were grown under bacteriologically controlled conditions in a greenhouse under natural light supplemented with artificial light (16/8 $\mathrm{h}$ day/night, at $25 / 18^{\circ} \mathrm{C}$ ). 21 days post inoculation (dpi) plants were harvested and roots were examined for typical red nodules.

In order to test the symbiotic capabilities of strains obtained in Chapters 2 and 3 , plant experiments were carried out in square plates containing $\mathrm{N}$-free Fahraeus agar medium $\left(\mathrm{CaCl}_{2} 0.1 \mathrm{~g} \cdot \mathrm{L}^{-1}, \mathrm{MgSO}_{4} \cdot 7 \mathrm{H}_{2} \mathrm{O} 0.12 \mathrm{~g} \cdot \mathrm{L}^{-1}, \mathrm{KH}_{2} \mathrm{PO}_{4} 0.1 \mathrm{~g} \cdot \mathrm{L}^{-1}, \mathrm{Na}_{2} \mathrm{HPO}_{4} 0.15\right.$ $\mathrm{g} \cdot \mathrm{L}^{-1}$, Ferric citrate $0.005 \mathrm{~g} \cdot \mathrm{L}^{-1}, \mathrm{MnSO}_{4} \cdot 7 \mathrm{H}_{2} \mathrm{O} 2.03 \mathrm{mg} \cdot \mathrm{L}^{-1}, \mathrm{ZnSO}_{4} \cdot 7 \mathrm{H}_{2} \mathrm{O} 0.22 \mathrm{mg} \cdot \mathrm{L}^{-1}$, $\mathrm{CuSO}_{4} \cdot 5 \mathrm{H}_{2} \mathrm{O} 0.08 \mathrm{mg} \cdot \mathrm{L}^{-1}, \mathrm{H}_{2} \mathrm{MoO}_{4} \cdot \mathrm{H}_{2} \mathrm{O} 0.08 \mathrm{mg} \cdot \mathrm{L}^{-1}, \mathrm{H}_{3} \mathrm{BO}_{3} 0.57 \mathrm{mg} \cdot \mathrm{L}^{-1} ; \mathrm{pH} 6.5$, agar $1 \%$ (Fåhraeus, 1957)). Surfaced-sterilize vetch seedlings were inoculated with $200 \mu \mathrm{l}$ of early stationary phase RIv cultures. Plants were grown in a growth chamber (16/8 $\mathrm{h}$ day/night, $22^{\circ} \mathrm{C}$ ) during 4 weeks, after which plates were examined for typical red nodules.

\subsubsection{Mesocosm experiment}

Trying to mimic repeated cycles of plant growth and inoculation that happen in the field, a controlled mesocosm, medium-term experiment was carried out with $P$. sativum, L. culinaris, $V$. faba and $V$. sativa used as trap plants. The experiment was carried out in rectangular pots $(40 \times 22 \times 30 \mathrm{~cm})$, filled with a mixture $(1: 1)$ of $P 1$ soil and sterile vermiculite. Four $V$. faba, eight $V$. sativa, and six $P$. sativum and $L$. culinaris germinated seeds were used per pot, and two pots were used per plant treatment. Each seedling was watered with $15 \mathrm{ml}$ of water two days per week, every two weeks it was substituted with Jensen solution. After three weeks, roots were collected and washed, plants from the same pot were pooled, roots were surface-sterilized (1 $\mathrm{min}$ ethanol $70 \%, 3$ min 1:4 bleach) and homogenized in 1:10 (w/v) PBS $\left(\mathrm{NaH}_{2} \mathrm{PO}_{4} 0.45 \mathrm{M}\right.$, $\begin{array}{lllllll}\mathrm{Na}_{2} \mathrm{HPO}_{4} & 0.06 \mathrm{M} \text { and Tween20 } & 0.02 \%) & \text { containing } 1: 3 & (\mathrm{w} / \mathrm{v}) & \text { PVPP }\end{array}$ (polyvinylpolypyrrolidone). Samples were centrifuged $1 \mathrm{~min}$ at $1,000 \times g$ in order to remove plant material and PVPP. The supernatant was used as inoculum of the next cycle for each pot. Inoculum samples and soil 1:10 (w/v) dilutions from each pot were maintained at $-80^{\circ} \mathrm{C}$ in $20 \%$ of glycerol for long-term storage. Plants on sterile vermiculite without soil were used as negative nodulation controls. In parallel, a trap inoculum experiment for each pot was carried out in Leonard jars containing Jensen's 
solution with three surface-sterilized seedlings per jar. Seedlings were inoculated with $1 \mathrm{ml}$ of 1:50 (v/v) inoculum dilution, plants harvested after 3 weeks, and roots stored at $-80^{\circ} \mathrm{C}$ for endosymbiont isolation. The experiment was repeated along five cycles, after which roots from each pot were harvested, washed and stored at $-80^{\circ} \mathrm{C}$ for endosymbiont isolation.

\subsection{MICROBIOLOGICAL TECHNIQUES}

\subsubsection{Preparation of cultures for Pool-Seq}

The bacterial isolates listed in Tables M2, M3 and M4 were selected for each PoolSeq sample. Each of those isolates was independently grown on YM broth for two days and each culture was adjusted to an $\mathrm{OD}_{600 \mathrm{~nm}}=2$. Portions $(1.5 \mathrm{ml})$ of cultures from isolates from the same sample were pooled together and centrifuged (10 min, 5,000 $x$ $g$ ). The cell pellet obtained from the combined cell suspension was used to extract genomic DNA (Wilson, 2001). DNA samples obtained from these pooled cultures were quantitated by Nanodrop (Thermo Scientific) spectrophotometry and Qubit $\circledast$ (Life Technologies) fluorometry.

\subsubsection{Isolation of rhizobial strains from soil}

The $\mathrm{P} 1$ soil was diluted $(1: 10, \mathrm{w} / \mathrm{v})$ in a buffer $\left(\mathrm{NaCl} 0.1 \mathrm{~g} \cdot \mathrm{L}^{-1}, \mathrm{KH}_{2} \mathrm{PO}_{4} 0.1 \mathrm{~g} \cdot \mathrm{L}^{-1}\right.$, $\mathrm{MgSO}_{4} 0.2 \mathrm{~g} \cdot \mathrm{L}^{-1} ; \mathrm{pH} 6.8$ ) and shaken for $2 \mathrm{~h}$ at $200 \mathrm{rpm}, 28^{\circ} \mathrm{C}$. This suspension was serially diluted and plated on MNBP agar medium. Plates were incubated for 4 days at $28^{\circ} \mathrm{C}$, and colonies (white and pink) were picked and replicated on MNBP and LB agar plates and incubated at $28^{\circ} \mathrm{C}$ for 2 days. Colonies that grew on MNBP and not on LB were picked and replicated on MNBP, YM and LB agar plates and incubated for 2 days at $28^{\circ} \mathrm{C}$. Those colonies that grew on MNBP and YM and not in LB were streaked for single colonies on YM plates, and those single colonies were re-checked for inability to grow on LB. Selected bacteria were grown on YM broth at $28^{\circ} \mathrm{C}, 200 \mathrm{rpm}$ until they reached stationary phase. Portions of these cultures were preserved at $-80^{\circ} \mathrm{C}$ in $20 \%$ glycerol. 


\subsection{NUCLEIC ACID METHODS}

\subsubsection{Nucleic acid extraction methods}

For PCR amplifications, RL genomic DNA was extracted from cultures grown in TY using DNeasy Blood \& Tissue Kit columns (QIAGEN Ltd.); a faster procedure was also used: DNA extraction was achieved through recovery of culture from a plate with a toothpick, followed by addition of $20 \mu$ of lysis solution $(0.05 \mathrm{M} \mathrm{NaOH}, 0.25 \%$ SDS). Samples were incubated $15 \mathrm{~min}$ at $90^{\circ} \mathrm{C}$, with a subsequent addition of $100 \mu$ l of water and two sequential steps of centrifugation, after which DNA was recovered from the supernatant. A CTAB (cetyl trimethyl ammonium bromide) bacterial genomic DNA isolation method was employed for total genome sequencing (Wilson, 2001). DNA concentration was estimated by loading $2 \mu \mathrm{l}$ of DNA in a $1 \%$ agarose gel and comparing intensities to those from calibrated standards, or by using Qubit 82.0 fluorometry (Life Technologies). Sample quality was determined by Nanodrop (Thermo Scientific) spectrophotometry.

\subsubsection{Amplification methods}

\subsubsection{Standard PCR}

Purified DNA was used as template for PCR amplifications. Standard PCRs were carried out with Taq DNA polymerase (Biotools) in $25 \mu$ l. Following PCR amplification, unincorporated primers and dNTPs were removed either directly from PCR mixes or by gel electrophoresis followed by band purification using NucleoSpin ${ }^{\circledR}$ Gel and PCR Clean-up (Macherey-Nagel).

\subsubsection{Electrophoresis}

DNA fragment size was checked by electrophoresis, using horizontal $1 \%$ agarose gels submerged in TBE buffer (Sambrook and Russell, 2001). DNA samples were prepared with $1 / 6$ volume of loading buffer (glycerol 30\%, Orange G (Sigma) $0.25 \%$ ). Gels were stained with SYBR® safe (Invitrogen) and visualized with a Gel Doc EZ system (Bio-Rad). Fragment sizes were estimated by comparing their rates of migration to those from the reference pattern for commercial 100 bp or $1 \mathrm{~kb}$ DNA ladders (Nippon Genetics). 


\subsubsection{Sequencing methods}

\subsubsection{Sanger}

Sequencing reactions were outsourced to Stabvida (Lisbon, Portugal), and sequence chromatograms were analysed using 4Peaks $\vee 1.7$ software (Nucleobytes $B$. V. Amsterdam, The Netherlands). Nucleotide and protein database searches were done using BLAST (Basic Local Alignment Search Tool, (Altschul et al., 1990)).

\subsubsection{Pool-Seq}

The pooled DNA rhizobial samples obtained in Chapter 1 and 2, were outsourced to BGI-Hong Kong (Illumina Hi-Seq 2000, 180 bp PE libraries, 100 bp reads, 12 Mreads), who provided clean reads. The pooled DNA rhizobial samples obtained in Chapter 3 were also outsourced (Illumina MiSeq V3, 600 bp PE libraries, 300 bp reads, 1 Mreads, ASU Genomics Core, Tempe, AZ for pea ${ }_{A}$; for the remaining Pool-Seqs Illumina MiSeq V2, 500bp PE libraries, 250 bp reads, 1-2 Mreads, Microseq Enterprise, Las Vegas, NV).

\subsubsection{Whole genome sequencing}

Individual rhizobial genomes obtained in Chapter 3 were sequenced externally (Illumina MiSeq v.3, 600 bp PE libraries, 300 bp reads, from 0.6 to 1.3 Mreads; ASU Genomic Core, Tempe, AZ).

\subsection{BIOINFORMATIC METHODS}

\subsubsection{Phylogenetic analysis}

Multiple sequence alignments were performed using MUSCLE algorithm (Edgar, 2004). Phylogenetic analyses were conducted using MEGA 6 (Tamura et al., 2013). The neighbour-joining statistical method and the Kimura two-parameter model were used for all the genes. Phylogenetic trees were bootstrapped with 1,000 replicates. 


\subsubsection{Pool-Seq analysis}

All subsequent bioinformatics analyses were conducted in-house as previously described (Jorrín and Imperial, 2015a). Reads were quality filtered with Trimmomatic (Bolger et al., 2014) and aligned against the Rhizobium leguminosarum bv viciae 3841 reference genome (GenBank accession No. PRJNA344, (Young et al., 2006)), as well as $R$. leguminosarum bv. viciae UPM791 genome (Sanchez-Cañizares et al., in preparation), $R$. leguminosarum bv viciae WSM1455 genome (GenBank accession No. PRJNA63183 (Reeve et al., 2015)) and R. leguminosarum bv viciae WSM1481 genome (GenBank accession No. PRJNA84327, (Reeve et al., 2015)), with Bowtie2 (Langmead and Salzberg, 2012), using very-sensitive standard parameters in end-toend mode (-D 20, $-\mathrm{R} \mathrm{3,-N} \mathrm{0,-L} \mathrm{20,-IS,} \mathrm{1,} \mathrm{0.50).} \mathrm{Output} \mathrm{from} \mathrm{the} \mathrm{alignment} \mathrm{was}$ transformed with Samtools ( $\mathrm{Li}$ et al., 2009), and Single Nucleotide Polymorphisms (SNPs) were detected with VarScan 2.3.6 (Koboldt et al., 2012). SNPs were called only if they satisfied the following conditions: minimum coverage of $10 \%$, minimum frequency of 0.1 and Fisher Exact Test $p$-values of observed reads vs. expected nonvariant under 0.01 . The genetic flow among samples was studied by calculating pairwise Wright's fixation indices $\left(F_{\mathrm{ST}}\right)$ for all SNPS, as calculated with the Popoolation2 package (Kofler et al., 2011b). Those SNPs with pairwise $F_{\mathrm{ST}}>0.1$ and $p<0.05$ were considered to provide a significant level of differentiation between populations, and SNPs with pairwise $F_{S T}>0.25$ and $p<0.05$ were considered to provide a very significant level of differentiation between populations (Wright, 1978, Barreiro et al., 2008).

For graphic representation of global SNP data, the output list of SNPs for each subpopulation obtained after VarScan2 analysis was used as input to calculate Euclidean distances (dissimilarity) with the SPSS package (IBM, v. 20.2). The dissimilarity matrix was subjected to multi-dimensional scaling with the SPSS PROXCAL module and predicted coordinates were plotted in a two-dimensional space with KaleidaGraph (Synergy Software, v. 4.5.1). For graphic representation of pairwise $F_{\mathrm{ST}}$ matrixes the multi-dimensional scaling ("cmdscale") R-package was used (www.rproject.org), and predicted coordinates were plotted in a two dimensional space with KaleidaGraph (Synergy Software, v. 4.5.1).

Other results were graphically analysed with SeqMonk (http://www.bioinformatics.babraham.ac.uk /projects/ seqmonk/), IGV (Robinson et al., 2011), and Qualimap v 2.1.3 (Okonechnikov et al., 2015). Reference sequence coverage was expressed as number of reads, RPKM or BPKM. RPKM values were calculated as: number of reads ${ }^{*} 10^{3 *} 10^{6} /$ length*total number of reads for each sample 
(Mortazavi et al., 2008) and BPKM values were calculated as: number of pair bases $* 10^{3 *} 10^{6} /$ length* total number of bases for each sample (Yang et al., 2011). \% $\mathrm{G}+\mathrm{C}$ composition values along sequences were calculated with a custom script. For both RPKM coverage and $\% G+C$ composition, sliding window sizes of 1,000 bp for replicon or $100 \mathrm{bp}$ bp for cluster analyses were used.

The graphical representation of heatmaps was rendered with the "pheatmap" Rpackage (www.r-project.org).

The web tool InteractiVenn (http://www.interactivenn.net/) was used to construct the Venn diagrams (Heberle et al., 2015).

\subsubsection{Genome Assembly}

Reads were quality filtered with Trimmomatic (Bolger et al., 2014) and assembled with SPAdes $\vee$ 3.5.0 (Bankevich et al., 2012). The resulting assemblies were improved with the PAGIT package (Swain et al., 2012), it uses ABACAS (Assefa et al., 2009) for contig ordering using $R$. leguminosarum bv. viciae 3841 as reference genome, IMAGE (Tsai et al., 2010) for closing gaps and iCORN (Tsai et al., 2010) for error correction. Finally, QUAST (Gurevich et al., 2013) was used in order to obtain genome assemblies statistics.

\subsubsection{Genome Annotation}

Assembled genomes were annotated with Prokka package (Seemann, 2014), ORFs (Open Reading Frame) were predicted with Prodigal (Hyatt et al., 2010), rRNAs were predicted with RNAmer (Lagesen et al., 2007) and the detection of tRNAs was performed with ARAGORN (Laslett and Canback, 2004). 


\subsubsection{Genomic Comparison}

The genomes were compared at different levels: to study synteny among specific clusters, a SYBIL (Crabtree et al., 2007) implementation within the CLoVR (Angiuoli et al., 2011) web local server was used. Pairwise alignments between genomes were performed with Nucmer package (Kurtz et al., 2004). In order to study genomic identity and phylogenomic relationship among strains, Average Nucleotide Identity (ANI) using MUMmer as alignment algorithm (ANIm), was calculated with the JSpecies package (Richter and Rosselló-Móra, 2009). Distances were calculated as 1ANI and trees computed by the UPGMA (Sneath and Sokal, 1973) method using MEGA 6 package. The exclusive genes for each host mesocosm-selected genomes were studied using the CMG biotools package (Vesth et al., 2013), the threshold for protein homology was $70 \%$ identity and $70 \%$ of length. 
4. CHAPTER 1: Population Genomics Analysis of Legume Host Preference for Specific Rhizobial Genotypes In the Rhizobium leguminosarum bv. viciae Symbioses ${ }^{1}$

\footnotetext{
${ }^{1}$ This chapter is based on Jorrín B, Imperial J. 2015. Population genomics analysis of legume host preference for specific rhizobial genotypes in the Rhizobium leguminosarum bv. viciae symbioses. Molecular Plant-Microbe Interactions, 28: 310-318.
} 



\subsection{INTRODUCTION}

Many legumes are able to establish a nitrogen-fixing root endosymbiosis with members of a number of proteobacterial groups collectively known as the rhizobia (Vanrhijn and Vanderleyden, 1995). The importance of this symbiosis for Agriculture and for the $\mathrm{N}$ cycle in the biosphere is widely recognized (Graham and Vance, 2003, Herridge et al., 2008). Rhizobia fix nitrogen within a specific organ, the legume root nodule, which represents the outcome of a highly complex developmental program where both plant and bacteria exchange molecular signals encoded by nodulation and symbiosis-specific genes (Hirsch, 1992, Denarie et al., 1996, Long, 1996, Gage, 2004 , Oldroyd et al., 2011). As a result of this molecular signal exchange, legume-rhizobial symbioses are highly specific, and only certain rhizobia can establish effective diazotrophic symbioses with any given legume (Denarie et al., 1996, Long, 1996, Oldroyd et al., 2011). There are exceptions to this high specificity in the legumerhizobial symbioses, such as in the case of some tropical legumes that exhibit promiscuous nodulation by a large number of different rhizobia (Perret et al., 2000, Martinez-Romero, 2003), or in the case of certain rhizobia that are equipped with different sets of nodulation genes specific for different plants (Perret et al., 2000, Koch et al., 2010).

A different situation arises when a given rhizobium equipped with just one set of symbiotic and specificity determinants can establish effective symbioses with different legume hosts. This is the case of Rhizobium leguminosarum bv. viciae. This bacterium is able to establish effective symbioses with members of the Pisum, Vicia, Lens and Lathyrus genera, all within the tribe Fabeae (Doyle and Luckow, 2003) and contains one set of nodulation and nitrogen fixation genes harboured in a plasmid responsible for the symbiotic genotype (Sym plasmid; (Surin and Downie, 1989, Young et al., 2006). With some exceptions (Mutch et al., 2003), most $R$. leguminosarum bv. viciae isolates containing a Sym plasmid are able to efficiently nodulate members of all four plant genera above. However, it has been suggested that, within R. leguminosarum bv. viciae, some genotypes exist that are better adapted to specific plants, and that the plant prefers, and thus selects for, these genotypes among those available in a given soil. Evidence in favor of this plant selection hypothesis has been obtained in the past through the use of restriction fragment length polymorphism analysis of PCR-amplified (PCR-RFLP) symbiotic and non-symbiotic molecular markers from isolates obtained from nodules of different plant genera (Louvrier et al., 1996, Palmer and Young, 2000, Laguerre et al., 2003, Depret et al., 2004, Mutch and Young, 2004). These studies relied on just a few, arbitrarily selected markers, and provided little information on the 
nature of the genotypes selected by specific plants or on the basis for their selection. We have now taken advantage of the availability of both cost-effective next generation sequencing technologies and a reference $R$. leguminosarum bv. viciae genomic sequence (Young et al., 2006) to reappraise the possible selection of specific $R$. leguminosarum bv. viciae genotypes by their different legume plant hosts. Towards this aim we have used a population genomics approach (Futschik and Schloetterer, 2010, Kofler et al., 2011a, Kofler et al., 2011b) that we have adapted to the study of rhizobial endosymbiont populations (Jorrín and Imperial, 2015a). We use this methodology to genomically compare rhizobial populations selected by Pisum sativum, Lens culinaris, Vicia sativa and $V$. faba plants from a well- characterized soil, and provide genomic evidence for plant-determined enrichment of specific $R$. leguminosarum bv. viciae populations. 


\subsection{RESULTS}

\subsubsection{Plant-selected populations}

Plant-selected $R$. leguminosarum bv. viciae populations consisted of one hundred isolates obtained, one each, from surface-sterilized root nodules excised from trap plants inoculated with our experimental P1 soil. Isolates were plate-purified, re-tested for symbiotic properties, and stored. Four populations were obtained: pea (Pisum sativum), lentil (Lens culinaris), vetch (Vicia sativa) and fava bean (V. faba).

\subsubsection{Next generation sequencing of pooled, plant-selected rhizobial populations}

DNAs from pooled samples of each of the four different plant-selected $R$. leguminosarum bv. viciae populations were submitted to next-generation sequencing (Pool-Seq) so that, on average, each of the one hundred genomes was sequenced to 2-3x coverage (Illumina HiSeq2000, 180 bp PE libraries, 100 bp reads, 12 Mreads; BGI, Hong Kong, China). This figure was chosen as a compromise between the higher sequencing costs at the time (2011) and the ability to detect sequences that might be present in just one of the isolates forming the population, and resulted in an average coverage of $200-240 \times$ for sequences present in the reference $R$. leguminosarum bv. viciae 3841 reference genome (Jorrín and Imperial, 2015a); data not shown). Individual sequencing reads from each population were recruited against the reference genome (Materials and Methods (Jorrín and Imperial, 2015a); and coverage results were normalized to correct for minor differences in sequencing depth among the four PoolSeq datasets.

\subsubsection{Presence of sequences from the $R$. leguminosarum bv. viciae reference genome}

\section{in Pool-Seq datasets}

The $R$. leguminosarum bv. viciae 3841 reference genome is multipartite and contains six large plasmids: pRL7 (151.5 kb) through pRL12 (870 kb), in addition to a $5,057 \mathrm{~kb}$ circular chromosome (Young et al., 2006). The presence and conservation of sequences from these seven replicons in the Pool-Seq datasets from P1 soil was investigated (Table 1.1). Sequences from the two smaller plasmids, pRL7 and pRL8 were minor components of the Pool-Seq datasets, suggesting that these plasmids, if present, are a minor component of the P1 soil $R$. leguminosarum bv. viciae population. The highest coverage was found with the chromosome (108.8-121.0 RPKM), followed by plasmids pRL12 (97.4-109.3 RPKM) and pRL9 (84.2-96.7 RPKM). The highest coverage values for the four largest plasmids ( $p R L 9-p R L 12)$ were found in the pea dataset, and the lowest in the lentil and vetch datasets. These data suggest that most 
Table 1.1. Average coverage (RPKM, Reads Per Kilobase per Million reads) of each reference genome (Rhizobium leguminosarum bv. viciae 3841) replicon by Pool-Seq DNA samples of the four plant-selected populations.

\begin{tabular}{lcccc}
\hline & Pea & Lentil & Fava bean & Vetch \\
\hline Chromosome & 121.0 & 112.9 & 108.8 & 120.3 \\
pRL7 & 8.3 & 7.6 & 6.8 & 6.3 \\
pRL8 & 7.5 & 6.2 & 6.7 & 6.0 \\
pRL9 & 95.2 & 84.6 & 96.7 & 84.2 \\
pRL10 & 90.1 & 74.1 & 86.5 & 74.8 \\
pRL11 & 90.0 & 79.3 & 84.9 & 80.7 \\
pRL12 & 109.3 & 97.4 & 98.4 & 99.9 \\
\hline
\end{tabular}

of the sequences in the chromosome and in the larger plasmids of the reference strain were present and well conserved in the P1 soil population, but also that differences in coverage exist among plant-selected populations. These differences could be explained in terms of differential divergence or absence of specific genes or regions from the reference genome in the genomes of members of the different plant-selected populations, and were analysed by examining the coverage of the reference genome replicons by recruited sequence reads of each of the four Pool-Seq datasets. Plots of coverage (RPKM) along replicon sequence are presented as Supplementary Figures 1.S1 to $1 . S 6$ for the chromosome, plasmids pRL7 and pRL8, pRL9, pRL10, pRL11, and $\mathrm{pRL} 12$, respectively. No striking differences in coverage among the four Pool-Seq datasets were observed for any of the replicons, although some regions from the reference genome appeared to be absent or overrepresented in the P1 soil populations. These regions are shown in Figure 1.1, where sequence reads from all four datasets were pooled and recruited against the reference genome replicons, and coverage and read sequence identity were plotted. Only two peaks of overrepresented contiguous sequences (coverage above 200 RPKM) appeared. These corresponded to chromosomal gene RL2002 (a DDE transposase; peak a), and to gene pRL90159 (a hypothetical protein; peak b), present in pRL9. Conversely, seven regions from the reference genome were absent or near absent in the P1 soil datasets. In addition, plasmids pRL8 and PRL7 were nearly absent, with very few reads being recruited to them. Low-coverage chromosomal regions were: Region 1 (RL0791- RL0841), region 2 (RL1869-RL1944), region 3 (RL2105-RL2195) and region 4 (RL3912-RL3956), spanning $51,76,91$, and 45 genes, respectively. These were genes of differing annotated functions, including enzymes, transmembrane or transport proteins, transcriptional factors, hypothetical proteins and other functions, including insertion sequences (region 1), transposases (regions 2 and 3), and phage proteins (region 4). The remaining three low-coverage regions were located, one each, in the three larger 
plasmids. Region 5 spanned 40 genes (pRL120440-pRL120479) and included a 14gene imp cluster $(i m p A-i m p N)$ that contains a type 6 secretion system. Region 6 contains 44 genes (pRL110145-pRL110188) of different annotated functions. Region 7 is present in the symbiotic plasmid pRL10, and spans 128 genes (pRL100011pRL100138) flanked by an integrase and a recombinase, suggesting that it could represent mobile DNA. In addition to genes annotated as encoding different enzyme activities and phage proteins, transposases and a recombinase/integrase, transcriptional regulators, a cold-shock protein and a repA gene are present in region 7. All seven low-coverage regions shown in Figure 1.1, $\mathrm{A}$, correspond to regions recruiting few, low identity reads from the datasets (Figure 1.1, C). However, with the exception of part of regions 3 and 4 , all seven regions recruit some reads at $100 \%$ identity (Figure 1.1, C), suggesting that these genomic regions are present in a minor fraction of the R. leguminosarum bv. viciae $\mathrm{P} 1$ soil population. The same situation was

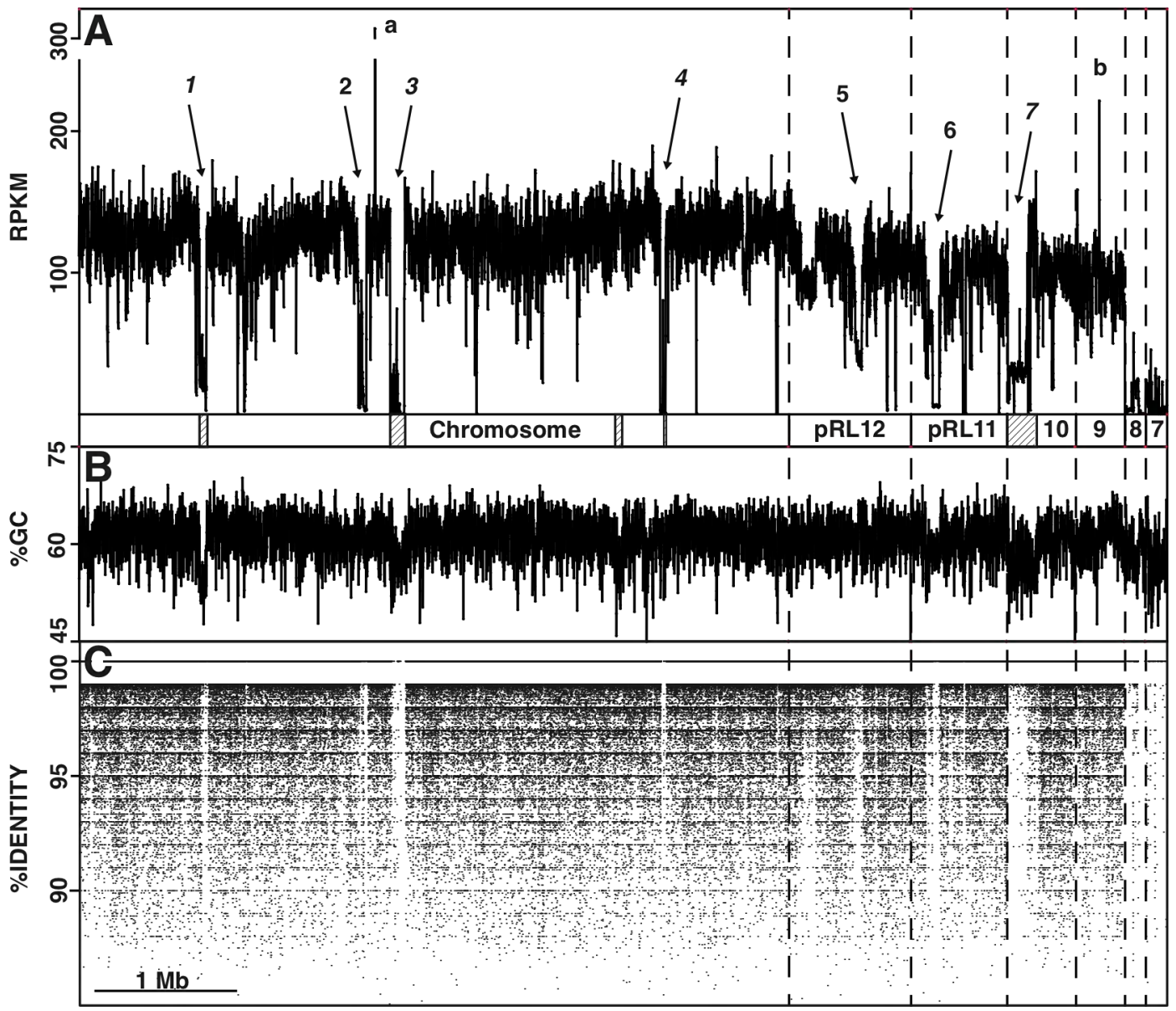

Figure 1.1. Conservation of $R$. leguminosarum bv. viciae 3841 reference genome sequences in the $\mathrm{P} 1$ soil rhizobial population. A. Coverage (RPKM) of genome regions by Bowtie2 recruited reads. The location and span of five low $\mathrm{G}+\mathrm{C}$ islands described by Young et al. (2006) in the reference genome are indicated by hatched bars. B. \%G+C plot of genomic regions. C. Identity (\%) of recruited reads. Sequences from all the reference genome replicons were concatenated as shown. Major under-represented and overrepresented regions in the $\mathrm{P} 1$ soil dataset are indicated by numbers and lowercase letters, respectively. 
found for plasmid pRL7 and part of plasmid pRL8 (Figure 1.1, C). Underrepresented regions often corresponded to low $\mathrm{G}+\mathrm{C}$ regions (Figure 1.1, B). This was clearly the case for regions $1,3,6$ and 7 . In addition, four of these regions $(1,3,4$, and 7 ) correspond to four of the five low $\mathrm{G}+\mathrm{C}$ genomic islands identified by $\mathrm{GC}$ analysis by Young et al. (2006).

Despite the large conservation of the reference genome within the P1 soil population, not all sequence reads from the subpopulation Pool-Seqs were recruited by the reference genome and, in fact, the unrecruited sequences unique to the P1 soil datasets constitute a large fraction of the reads $(15.8 \%, 22.2 \%, 18.1 \%, 22.8 \%$ for the pea, lentil, vetch, and fava bean subpopulations, respectively). Aside from possible artifactual sequences, these sequence reads probably arise from genomic regions present in the P1 soil population, but absent in the reference genome.

\subsubsection{Sequence polymorphisms in specific regions of Pool-Seq datasets}

Previous work on plant host selection of $R$. leguminosarum bv. viciae genotypes had centred on PCR-RFLP analyses of the nodD-F region (Laguerre et al., 2003, Mutch and Young, 2004). The Pool-Seq methodology allows the comparative study of population-linked polymorphisms at the single nucleotide level (SNP) for any gene or region of interest in the reference genome. We analyzed two contrasting regions: the chromosomal 16S rRNA-23S rRNA region, encoding conserved housekeeping rRNAs, and the symbiotic plasmid-encoded nod cluster, responsible for synthesis of the Nod factor, the main symbiotic signal.

\subsubsection{16S rRNA-23S rRNA region}

The reference $R$. leguminosarum bv. viciae 3841 genome contains three copies of the rRNA operon, all identical and located in the chromosome, with the structure: rRNA $16 S$ - tRNA lle - tRNA Ala - rRNA 23S - rRNA 5 S (Young et al., 2006). Plant-selected Pool-Seq datasets were recruited to this region, and coverage and SNPs were plotted along the region (Figure 1.2). The high level of coverage for all four plant-selected datasets, above 1,000 for $16 S$ and $23 S$ rRNAs, suggests that most rhizobia in these populations contain several copies of these genes, perhaps three, as in the reference strain or in other $R$. leguminosarum strains ( $R$. leguminosarum bv. trifolii WSM2304, Accession number PRJNA20179; R. leguminosarum bv. trifolii WSM1325, PRJNA20097; R. leguminosarum bv. viciae TOM, PRJNA199010; R. leguminosarum bv. trifolii SRDI943, PRJNA199021; R. leguminosarum bv. phaseoli 4292, PRJNA199148; $R$. leguminosarum bv. trifolii SRDI565, PRJNA199011; $R$. leguminosarum bv. viciae 248, PRJNA201173; $R$. leguminosarum bv. viciae 

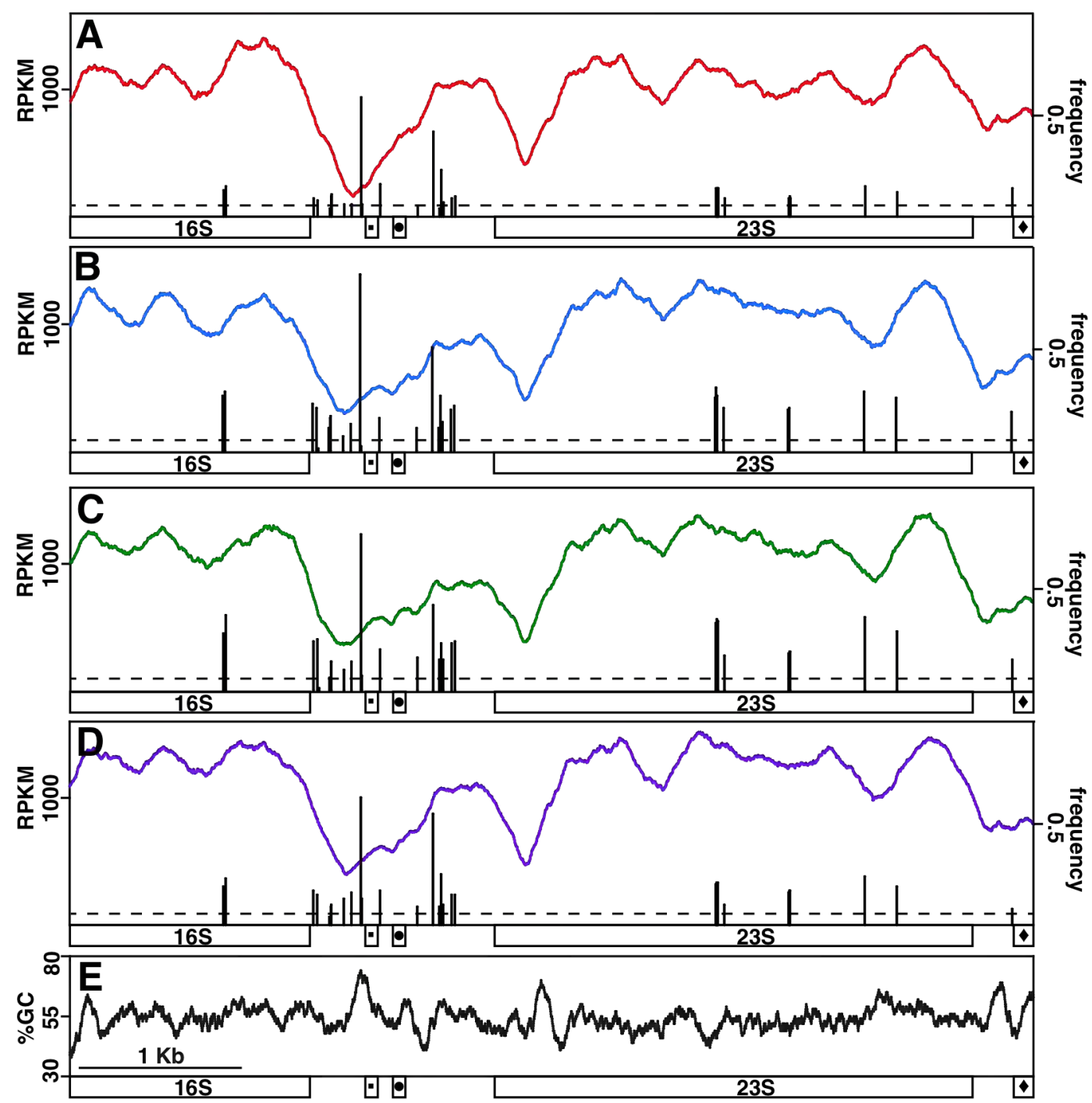

Figure 1.2. Conservation and diversity of the 16S-23S rRNA genomic region in the four plantselected rhizobial populations. A. Pea. B. Lentil. C. Fava bean. D. Vetch. Coverage (RPKM) along the reference genome sequence is indicated by a continuous line. SNP location and frequency are indicated by vertical lines. A discontinuous horizontal line indicates the $p<0.01$ threshold, which, in these experiments, corresponded to a coverage of $c a .5 \%$. The position of Ile-tRNA, Ala-tRNA and 5S rRNA in the reference genome sequence is indicated by boxes containing a small square a large dot and a diamond, respectively.

WSM1481, PRJNA199020; R. leguminosarum bv. trifolii CB782, PRJNA67103; $R$. leguminosarum bv. trifolii WSM1689, PRJNA62289; $R$. leguminosarum bv. trifolii WSM2012, PRJNA18209). Coverage of the reference sequence decreased drastically for all four plant-selected populations in the 16S-23S intergenic region, suggesting that parts of this region may be non-conserved or absent in rhizobia from the P1 soil. This was also true of the Ile- and Ala-tRNAs present in the 16S-23S intergenic region of the reference strain, especially for the lle-tRNA, which appeared to be nearly absent in the pea Pool-Seq dataset (Figure 1.2). Comparative genomic analysis of rRNA clusters from other sequenced $R$. leguminosarum strains (see above) indicated a generalized 
lack of conservation in the 16S-23S region, although lle- and Ala-tRNAs were present in most cases (data not shown). It is worth noting that decreases in sequence coverage also occurred in two intragenic regions of the 23S rRNA for all four Pool-Seq populations, although we do not currently have a clear explanation for this observation. One possibility is that the Illumina sequence technology could be affected by sequence composition, and Aird et al. (2011) showed that the PCR step in sequencing library preparation selects strongly against regions with anomalous $\% \mathrm{G}+\mathrm{C}$. Indeed, inspection of the $\% \mathrm{G}+\mathrm{C}$ plot (Figure 1.2, E) suggested that regions of low coverage are associated with high $\% \mathrm{G}+\mathrm{C}$ peaks. Alternating high- and low $-\% \mathrm{G}+\mathrm{C}$ regions were also common in 23S rRNA genes from other $R$. leguminosarum genomes (data not shown). Despite these anomalies, the overall coverage pattern for $16 S$ and $23 S$ rRNA sequences was very similar for all four plant-selected datasets.

As predicted, few single nucleotide polymorphisms were recorded in this region (Figure 1.2). Pairwise $F_{\mathrm{ST}}$ fixation indices were low $\left(F_{\mathrm{ST}}<0.1\right)$ with $p<0.001$ for all SNPs except for a single SNP in the pea/lentil comparison (data not shown), indicating that observed SNPs were not specific for any of the plant-selected populations. In all, 32 polymorphic sites along a 5,903 bp stretch (0.54\%) were observed, 2 in the $16 \mathrm{~S}$ rRNA gene, 11 in the 23S rRNA gene, and 19 in the 16S-23S intergenic region. Except for a specific SNP in the 16S-23S intergenic region, no SNP was present at frequencies higher than 0.5 , suggesting that the reference genome sequence represents the most abundant genotype for this region in the P1 soil rhizobial population. Some differences were observed in the relative frequency of these SNPs in the four populations, with lower frequencies in the pea population, which may be interpreted as suggesting a preference for the reference genome genotype, which was in fact isolated from a pea nodule (Young et al., 2006).

\subsubsection{2. nod cluster}

A similar analysis of Pool-Seq populations was carried out with the nod region. Figure 1.3 shows the read coverage and SNP distribution along a stretch of $13,462 \mathrm{bp}$ from symbiotic plasmid pRL10 that includes the nodTNMLEFDABCIJ genes. The coverage pattern was similar among the four plant-selected populations, but important differences were observed in the number and distribution of SNPs. A total of 455 polymorphic sites were recorded (3.4\%), with 409 in coding regions and 46 in intergenic regions. Overall, a large concentration of polymorphic sites was observed within the nodTMN and nodJ coding sequences. This included both synonymous and non-synonymous sites, with polymorphism frequencies of up to 0.5 with respect to the 

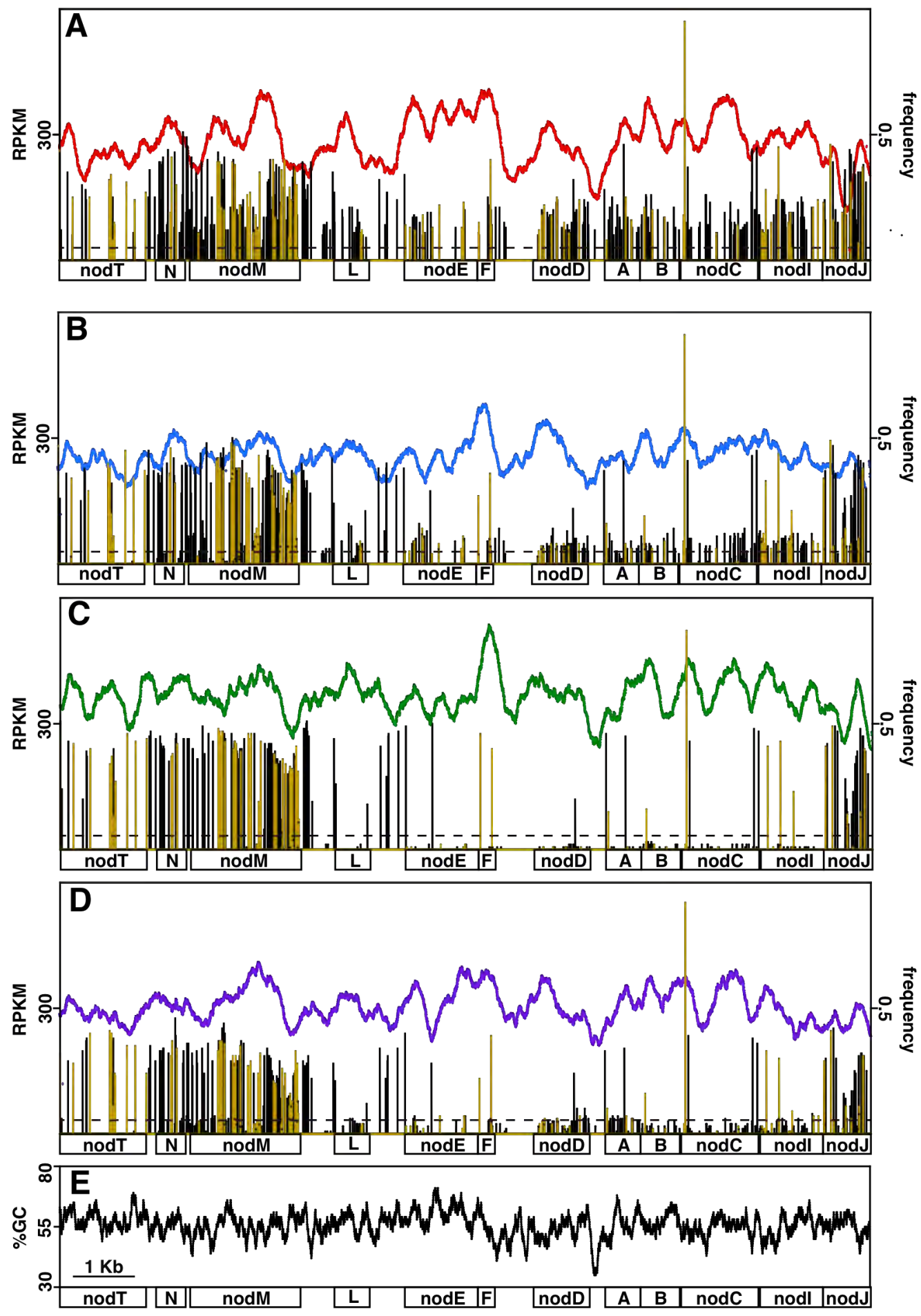

Figure 1.3. Conservation and diversity of the nod region in the four plant-selected rhizobial populations. A. Pea. B. Lentil. C. Fava bean. D. Vetch. Coverage (RPKM) along the reference genome sequence is indicated by a continuous line. SNP location and frequency are indicated by vertical lines. A discontinuous horizontal line indicates the $p<0.01$ threshold, which, in these experiments, corresponded to a coverage of $c a$. $5 \%$. E. \% G+C composition along the genomic region. 
reference genome, but with little difference among Pool-Seq datasets. Clear differences, however, were observed among plant-selected populations in the central nod cluster nodLEFDABCI, especially between the pea and fava bean datasets. Along this region, the fava bean dataset showed a small number of polymorphic sites and at low frequencies, except for a few that appeared in all four datasets and that probably characterize the P1 soil rhizobial population. In this respect, it is worth mentioning that a non-synonymous (Ala $>$ Ser) substitution $(G>T)$ at position 55 of the nodC coding region was present in all four populations at frequencies of ca. 0.9 and this could be taken as characteristic of the P1 soil nod cluster (although this substitution is present in many of the $R$. leguminosarum nodC sequences in GenBank; data not shown). In contrast, the pea dataset presented a large number of low (0.2-0.3) frequency SNPs within the central nod cluster. A similar pattern was observed for the lentil population, although the SNP frequencies were lower, whereas the vetch population displayed a pattern intermediate between those of lentil and fava bean. Significance analysis of the observed SNPs among plant-selected subpopulations was carried out using their pairwise $F_{\mathrm{ST}}$ indices (Supplementary Table 1.S1). Whereas no SNP with $F_{\mathrm{ST}}>0.1$ was observed for the lentil / fava, lentil / vetch or fava bean / vetch pairs, and only one for the pea / lentil pair, high (85) or intermediate (8) numbers were observed for the pea / fava bean and the pea / vetch pairs, respectively, thus confirming the genotypic differentiation among these subpopulations. As expected, all P1 soil-derived populations showed large differences with the reference strain 3841 (151-192 SNPs with $F_{\mathrm{ST}}>0.1$ and $\left.p<0.002\right)$.

\subsubsection{Genome-wide sequence polymorphisms in Pool-Seq datasets}

A comparative analysis of all single nucleotide polymorphisms in sequences recruited by the reference genome was carried out, and the data are summarized in Table 1.2. Similar total numbers of polymorphic sites were observed for all four PoolSeq P1 soil subpopulations, with the pea subpopulation exhibiting the lowest number $(214,467)$ and the fava bean subpopulation the highest $(299,099)$. These numbers correspond to $2.8-3.9 \%$ polymorphic sites and can probably be taken as characteristic of the $\mathrm{P} 1$ soil population. The levels of polymorphism are comparable, albeit somewhat lower, than those found within Rhizobium etli (4-6\%; (Acosta et al., 2011)). For each replicon they showed slight variations among subpopulations, with pRL7 and pRL8 showing the least polymorphisms $(0.5-1.0 \%)$. This is probably related to the very low abundance of these plasmids in the P1 soil population (Fig. 1). Significance analysis of the observed SNPs for the whole genome among plant-selected subpopulations was carried out using their pairwise $F_{\mathrm{ST}}$ indices (Supplementary Table 1.S2). An overall 
Table 1.2. Genome-wide abundance of single nucleotide polymorphism in Pool-Seq DNA samples of the four plant-selected populations ${ }^{a}$

\begin{tabular}{lcccc}
\hline & Pea & Lentil & Fava bean & Vetch \\
\hline Total & 214,647 & 269,749 & 299,099 & 261,282 \\
Total \% & 2.77 & 3.48 & 3.86 & 3.37 \\
Chromosome & 2.76 & 3.59 & 3.98 & 3.45 \\
pRL7 & 1.00 & 0.74 & 0.94 & 0.72 \\
pRL8 & 0.60 & 0.55 & 0.51 & 0.50 \\
pRL9 & 2.98 & 3.59 & 4.00 & 3.58 \\
pRL10 & 2.96 & 3.20 & 3.43 & 3.10 \\
pRL11 & 3.12 & 3.66 & 4.09 & 3.68 \\
pRL12 & 3.00 & 3.78 & 4.21 & 3.66 \\
\hline
\end{tabular}

${ }^{a}$ Expressed as total number and as percentages of each replicon and of the total reference genome sequenced.

pattern similar to that already observed with the nod region (see above) reappeared at the genomic level: very few significant SNPs $(1,264-1,593)$ were observed in pairwise combinations not involving the pea subpopulation, which showed a larger level of differentiation with the vetch and lentil subpopulations (2,485 and 3,998 significant SNPs, respectively), and especially with the fava bean subpopulation $(11,087$ significant SNPs). Again, all P1 soil-derived subpopulations showed large differences with reference strain 3841 (106,384 - 223,009 significant SNPs).

Nucleotide frequencies at all sites in the reference genome for each subpopulation were transformed into Euclidean distances (Supplementary Table 1.S3), subjected to multi-dimensional scaling and the two main coordinates plotted in a two-dimensional space (Supplementary Fig. 1.S7). The plot separated all four subpopulations among themselves and from the reference genome. The P1 population as a whole was set apart from the reference genome along dimension 1, whereas dimension 2 clearly separated the most divergent subpopulations, pea and fava bean. 


\subsection{DISCUSSION}

The fact that symbiotic Rhizobium leguminosarum bv. viciae strains, irrespective of their plant origin, are able to nodulate effectively all their legume hosts (Pisum, Lens, Vicia and Lathyrus) complicates studies aimed at establishing the existence of a preference, on the part of the legume host, for specific rhizobial genotypes, and previous studies have relied on arbitrarily selected genotypic markers (Louvrier et al., 1996, Palmer and Young, 2000, Laguerre et al., 2003, Depret et al., 2004, Mutch and Young, 2004). We have adapted to rhizobial populations (Jorrín and Imperial, 2015a) the Pool-Seq population genomics approach first developed by the Schloetterer lab for the genomic study of Drosophila populations (Futschik and Schloetterer, 2010, Kofler et al., 2011a, Kofler et al., 2011b) in order to approach this problem. As discussed, this methodology takes advantage of the existence of a $R$. leguminosarum bv. viciae reference genome (Young et al., 2006) and of the diminishing costs of next generation sequencing to genomically compare different populations without the need to choose any specific marker, in itself a limitation, or without the labour and expense associated with sequencing the genome of large numbers of isolates from the different populations (Jorrín and Imperial, 2015a).

The source of our $R$. leguminosarum bv. viciae populations was soil $\mathrm{P} 1$, well characterized for rhizobial populations in previous studies of plant preference for specific genotypes (Louvrier et al., 1996); Laguerre et al. 2003; G. Laguerre, personal communication). This allowed us to compare the results of our work to those studies. At the population level, the $\mathrm{P} 1$ soil symbiotic $R$. leguminosarum bv. viciae population showed an overall good genomic conservation with the reference genome, particularly in the chromosome, but also in the large plasmids. Despite this conservation, a few over- and under-represented regions were identified. In the first case, they correspond to specific genes (a DDE transposase and a hypothetical protein) that probably underwent duplication within the $\mathrm{P} 1$ population. In the second case, seven major regions under-represented in $\mathrm{P} 1$ soil correspond to groups of 14-127 genes that probably represent acquisitions of the reference genome for ecological adaptations to its habitat. It is noteworthy that four of these regions correspond to low $G+C$ genomic islands previously identified in the reference genome (Young et al. 2006). Among these low $\mathrm{G}+\mathrm{C}$ islands, the largest (region 7 ) is located in the symbiotic plasmid ( $p R L 10$ ), just upstream of the nod cluster. Among the underrepresented regions not clearly associated to low $\mathrm{G}+\mathrm{C}$, it is worth noting the presence of a type 6 secretion system in plasmid pRL12 (region 5). The two smaller plasmids, pRL7 and pRL8, also appeared to be strongly under-represented in the $\mathrm{P} 1$ soil population, although sequences 
recruited by these plasmids had low levels of polymorphism, suggesting that they are very strongly conserved within the small fraction of cells that carry them.

It is also important to mention that a sizeable number (15.8\%-22.8\%) of sequence reads from the Pool-Seq subpopulations was not recruited by the reference genome. Since many of these reads probably represent specific genes present in the P1 soil population and relevant for adaptation to specific ecological conditions in $\mathrm{P} 1$ soil, their nature and conservation among subpopulations are currently under study.

The Pool-Seq approach allows detailed analysis of single nucleotide polymorphisms for any specific region of interest within the reference genome. In this study we chose the highly conserved 16S-23S rRNA region and the symbiotic nodulation cluster (nod genes). Since ribosomal RNAs are subjected to high structural / functional constraints, polymorphisms in these molecules are kept to a minimum and reflect the evolutionary history of the taxons under study (Woese, 1987). In fact, rRNA sequence comparisons are at the root of present-day prokaryotic phylogenies (Woese et al., 1990). The $16 \mathrm{~S}$ and $23 \mathrm{~S}$ rRNA genes were highly conserved when comparing P1 soil subpopulations and the reference genome, although large differences appeared in the intergenic regions, including the lle- and Ala-tRNA genes located in the 16S-23S rRNA intergenic region. Apart from some distinct SNPs, a generalized decrease of coverage in this region probably means that it is absent in some of the members of the population. This could be especially true of the lle-tRNA gene, whose coverage practically disappears in the pea subpopulation. It is possible that the number, position and frequency of the observed SNPs and possible deletions in the intergenic regions may constitute a fingerprint of the soil population, and we are currently testing this in populations from other soils. However, none of the observed differences was specific of any of the plant-selected subpopulations, which suggests that, as expected, rRNA genes are neutral in this respect. The opposite situation was encountered in the analysis of the nod gene cluster. These genes are responsible for synthesis of the lipochitoolygosaccharide signal molecule (Nod factor) specifically recognized by the plant (Denarie et al. 1996), and clear differences were observed in the frequency of SNPs in this region, both for the P1 population as a whole and for each four plantselected P1 soil subpopulations. Two large polymorphic subregions, nodTMN and nodJ, differentiated the P1 population from the reference genome, irrespective of the plant-selected subpopulation. However, SNP analysis of the nodLEFDABCI region showed clear differences among plant subpopulations, with a large number of SNPs in the pea subpopulation, a very small number of SNPs in the fava bean subpopulation and intermediate results for the lentil and vetch subpopulations. It is tempting to interpret these results as meaning that fava bean plants are more restrictive regarding 
the nod genotype of their rhizobial symbionts than pea plants or, alternatively, that the nod genotype from the reference genome colonizes fava beans more efficiently, whereas none of the available genotypes shows a clear advantage in the colonization of pea plants. However, any further interpretation is hampered by limitations inherent to the Pool-Seq approach, where the strain origin of the different reads, and hence SNPs, is not known. For instance, for the pea subpopulation, where many lowfrequency SNP loci were observed in the nod region, it would be equally possible, in principle, that these SNPs are all present within the genomes of a small, highly polymorphic fraction of the population, or that, they are the sum of polymorphic sites scattered throughout most of the population. At any rate, the observed, contrasting nod pattern confirms and extends previous studies with nod $D$ and the nod $D-F$ intergenic region where isolates from fava bean nodules were shown to present lower diversity than isolates from pea nodules (Laguerre et al., 2003, Mutch and Young, 2004).

Irrespective of the neutral (such as the 16S-23S rRNA region) or selected (such as the nod region) nature of the specific genomic region considered, we hypothesized that any plant selection of rhizobial genotypes would be reflected in the number or distribution of SNPs at the level of the complete genome. Differences in the number and replicon distribution of SNPs for the four plant Pool-Seq datasets were observed (Table 2), although their biological significance is not clear.

Although this study and a previous report (Jorrín and Imperial, 2015a) represent the first Pool-Seq studies of rhizobial populations, other population genomics studies have been undertaken with rhizobia that reveal genomic adaptations to the host and suggest that host selection of rhizobial microsymbiont genotypes could be a general phenomenon in the legume-rhizobium symbiosis. Using low (0.4-1.2x) coverage genome sequencing of 12 Ensifer medicae strains isolated from Medicago lupulina nodules, Bailly et al. (2011) were able to identify population specific polymorphisms in conserved genes as well as putative population-specific genes when compared with the E. medicae WSM 419 reference strain (isolated from M. murex). More recently (Epstein et al. 2012; Sugawara et al. 2013), deep sequencing of up to 48 genomes from E. meliloti, E. medicae and three other Ensifer genospecies was used to study genomic adaptations to the plant host. These studies revealed not only that specific genes, among them nod genes and type III, IV and VI secretion systems, differ among rhizobial genospecies showing different plant specificities, but also that clear phenotypic interactions exist among rhizobial genotypes and $M$. truncatula host plant genotypes, a clear evidence for rhizobial genotype selection by the plant host. 
Our work exemplifies the potential of the Pool-Seq population genomics approach to study the selection of specific rhizobial genotypes by the host plant, but its limitations make it difficult to investigate in depth the exact nature of the selection, in particular the role of any specific genes in this selection. In order to solve this uncertainty we are currently carrying out genome sequencing of a representative subset of the plantselected rhizobial isolates. These genome sequences will allow a better characterization of the plant-specific genotype selection, particularly for those genes that are not present in the reference genome. 


\subsection{SUPPLEMENTARY MATERIAL}

Supplementary Table 1.S1. Pairwise comparison of SNPs among plant-selected rhizobial subpopulations and with the reference genome in the nod region. Numbers of significant SNPs $\left(F_{\mathrm{ST}}>0.1, p<0.02\right)$ are indicated

\begin{tabular}{llllll}
\hline & Pea & Lentil & Fava bean & Vetch & RIv3841 \\
\hline Pea & 0 & & & & \\
Lentil & 1 & 0 & & & \\
Fava bean & 85 & 0 & 0 & & \\
Vetch & 8 & 0 & 0 & 0 & \\
Rlv3841 & 192 & 169 & 175 & 151 & 0 \\
\hline Rlv3841: Rhizobium leguminosarum bv. viciae 3841 & &
\end{tabular}

RIv3841: Rhizobium leguminosarum bv. viciae 3841

Supplementary Table 1.S2. Pairwise comparison of SNPs among plant-selected rhizobial subpopulations and with the reference genome for the whole genome. Numbers of significant SNPs $\left(F_{\mathrm{ST}}>0.1, p<0.02\right)$ are indicated

\begin{tabular}{lccccc}
\hline & Pea & Lentil & Fava bean & Vetch & Rlv3841 \\
\hline Pea & 0 & & & & \\
Lentil & 3,998 & 0 & & & \\
Fava bean & 11,087 & 1,593 & 0 & & \\
Vetch & 2,485 & 1,503 & 1,264 & 0 & \\
Rlv3841 & 106,384 & 218,867 & 223,009 & 164,508 & 0 \\
\hline \multicolumn{7}{l}{ Rlv3841: Rhizobium leguminosarum bv. viciae 3841} & &
\end{tabular}

Supplementary Table 1.S3. Euclidean distances among plant-selected rhizobial subpopulations sequences derived from genome-wide SNP frequency dissimilarities

\begin{tabular}{lccccc}
\hline & Pea & Lentil & Fava bean & Vetch & Rlv3841 \\
\hline Pea & 0 & & & & \\
Lentil & 74.90 & 0 & & & \\
Fava bean & 90.26 & 52.80 & 0 & & \\
Vetch & 54.88 & 55.27 & 61.69 & 0 & \\
Rlv3841 & 168.47 & 198.42 & 206.93 & 180.61 & 0 \\
\hline Rlv3841: Rhizobium leguminosarum bv. viciae 3841 & &
\end{tabular}



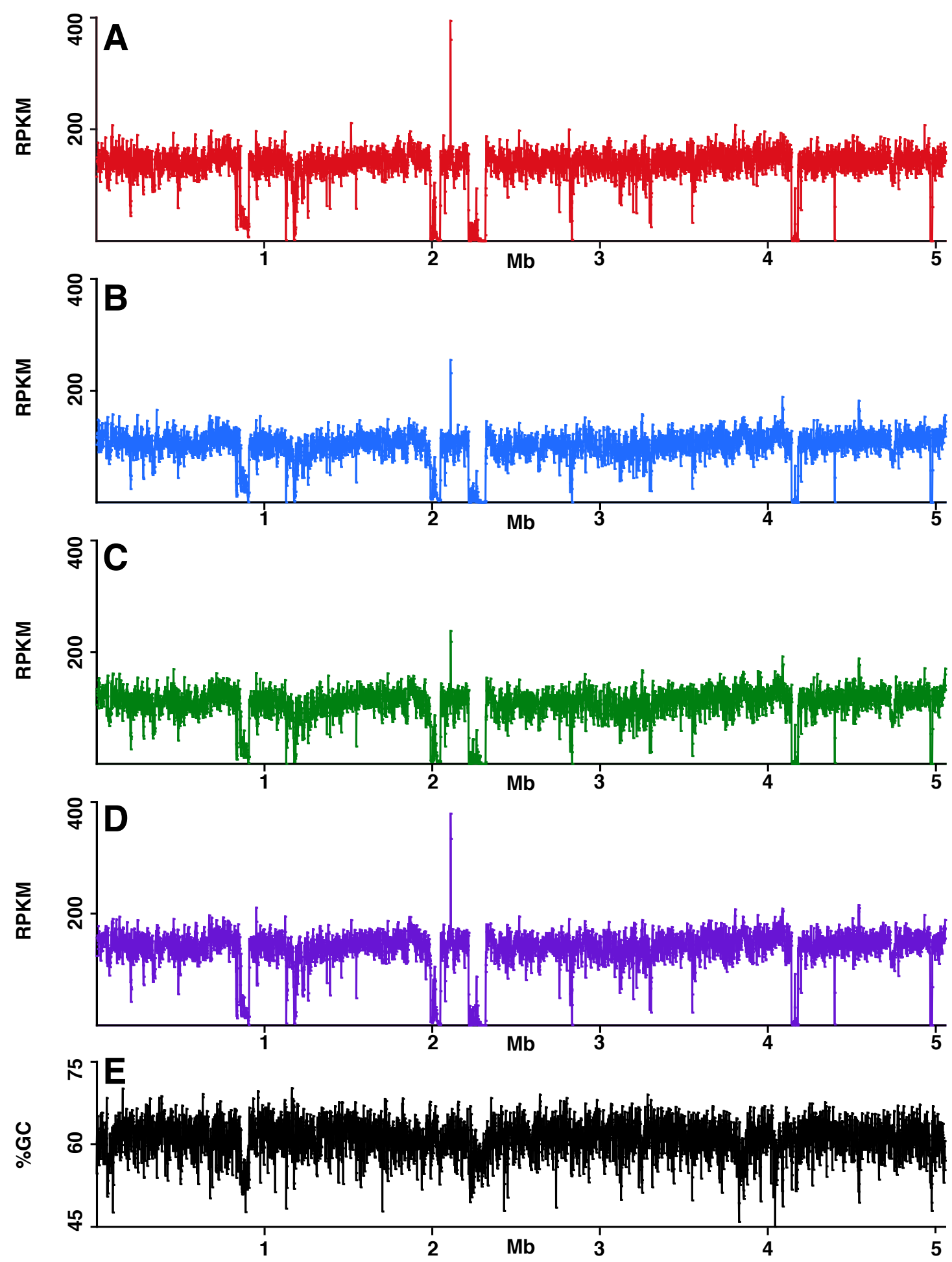

Supplementary Figure 1.S1. Conservation of reference genome chromosome in the four plant-selected rhizobial populations. A. Pea. B. Lentil. C. Fava bean. D. Vetch. E. \%GC. RPKM coverage along the reference genome sequence is indicated by a continuous line. 

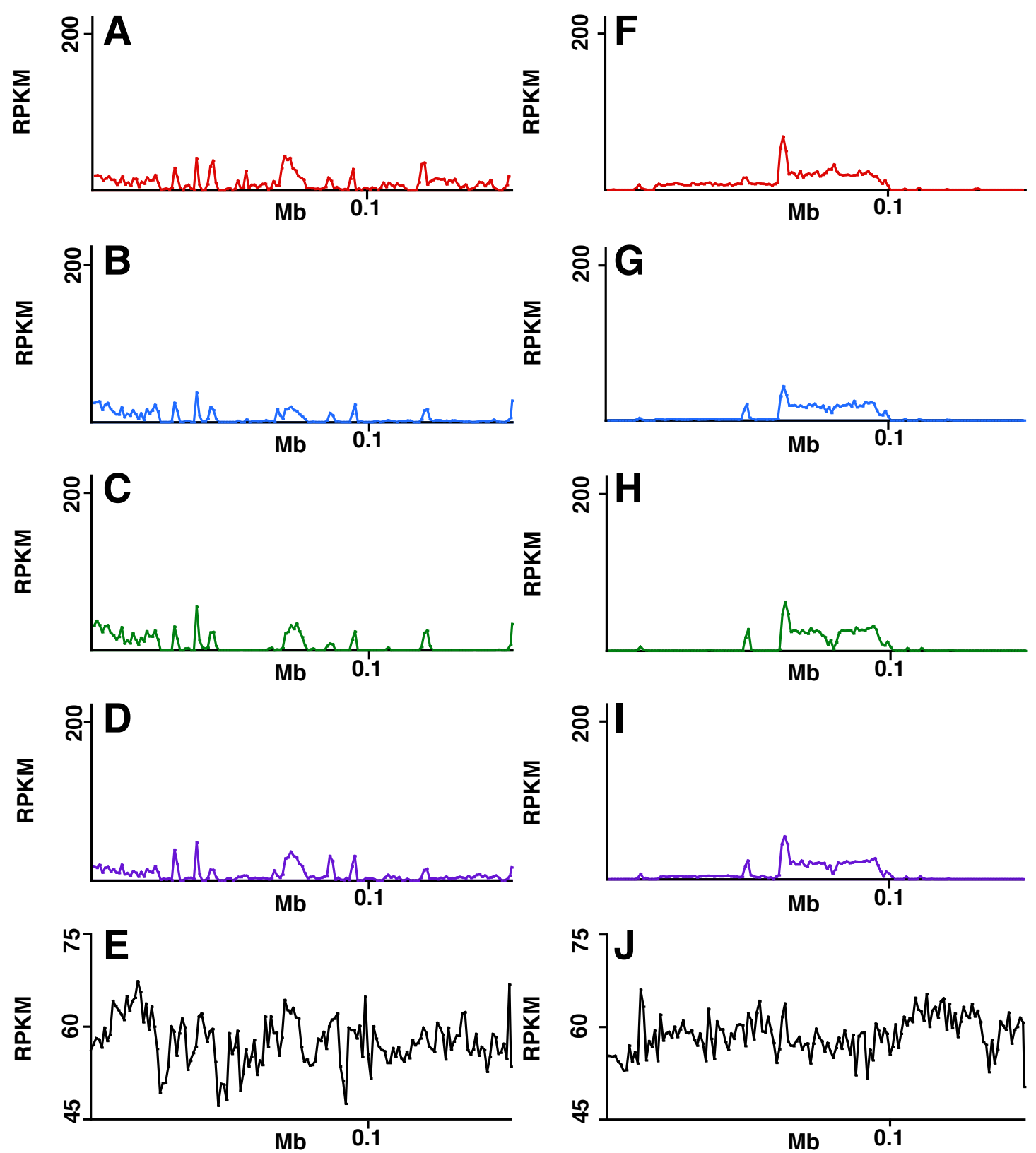

Supplementary Figure 1.S2. Conservation of small plasmid replicons from the reference genome in the four plant-selected rhizobial populations. Plasmid pRL7: A. Pea. B. Lentil. C. Fava bean. D. Vetch. E. \%GC. Plasmid pRL8: F. Pea. G. Lentil. H. Fava bean. I. Vetch. J. $\% G C$. RPKM coverage along the reference genome sequence is indicated by a continuous line. 

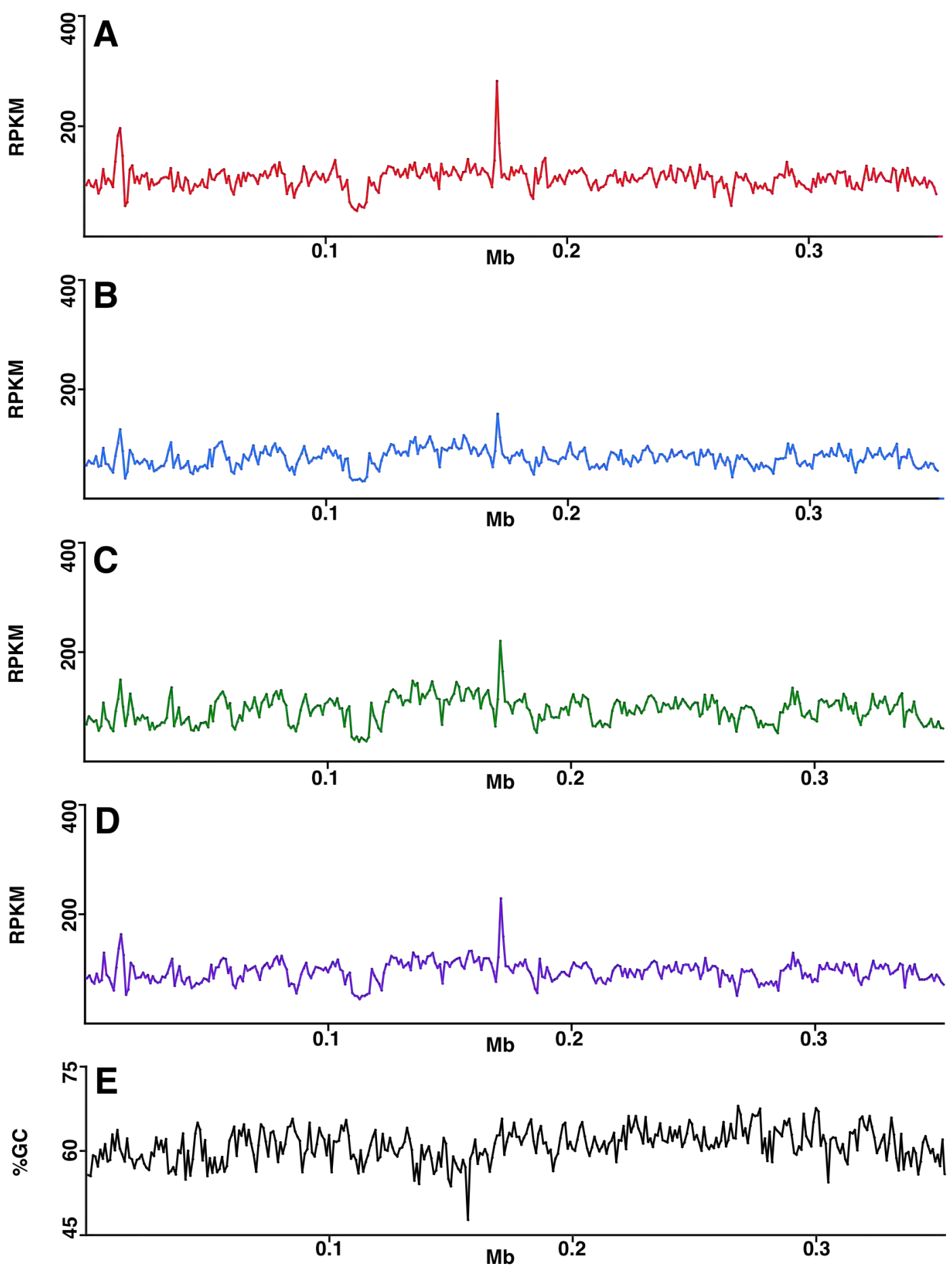

Supplementary Figure 1.S3. Conservation of reference genome plasmid pRL9 in the four plant-selected rhizobial populations. A. Pea. B. Lentil. C. Fava bean. D. Vetch. E. \%GC. RPKM coverage along the reference genome sequence is indicated by a continuous line. 

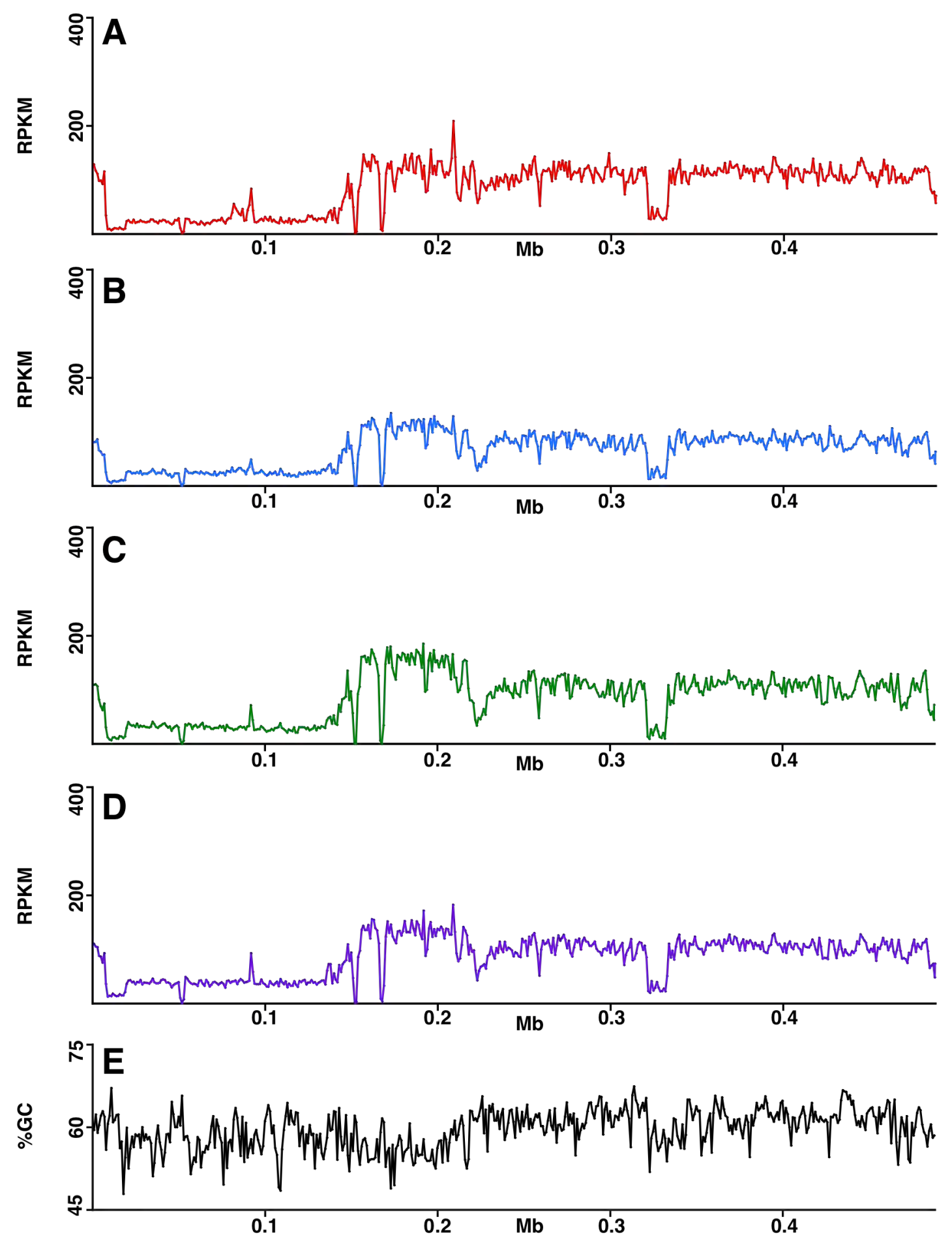

Supplementary Figure 1.S4. Conservation of reference genome plasmid pRL10 in the four plant-selected rhizobial populations. A. Pea. B. Lentil. C. Fava bean. D. Vetch. E. \%GC. RPKM coverage along the reference genome sequence is indicated by a continuous line. 

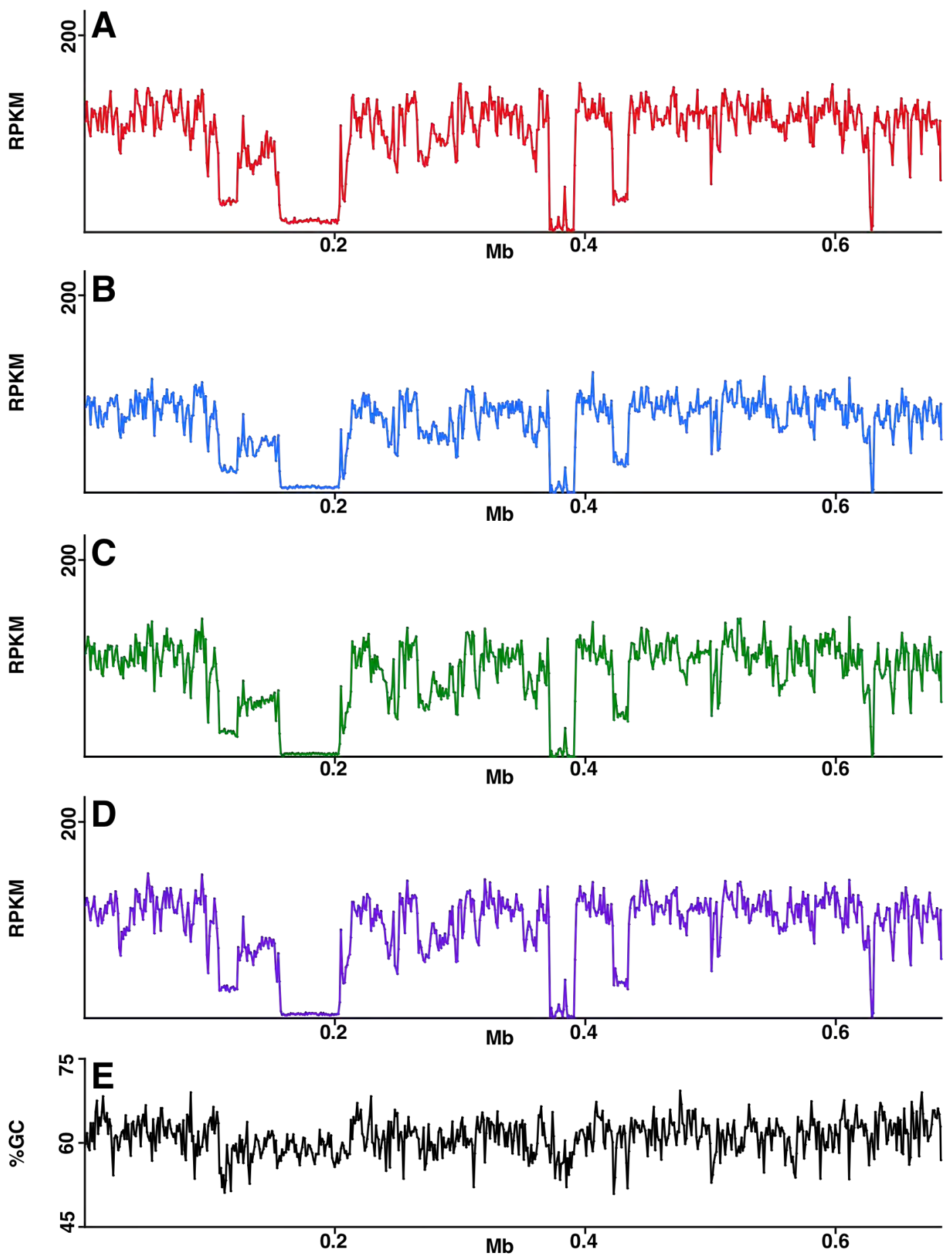

Supplementary Figure 1.S5. Conservation of reference genome plasmid pRL11 in the four plant-selected rhizobial populations. A. Pea. B. Lentil. C. Fava bean. D. Vetch. E. \%GC. RPKM coverage along the reference genome sequence is indicated by a continuous line. 

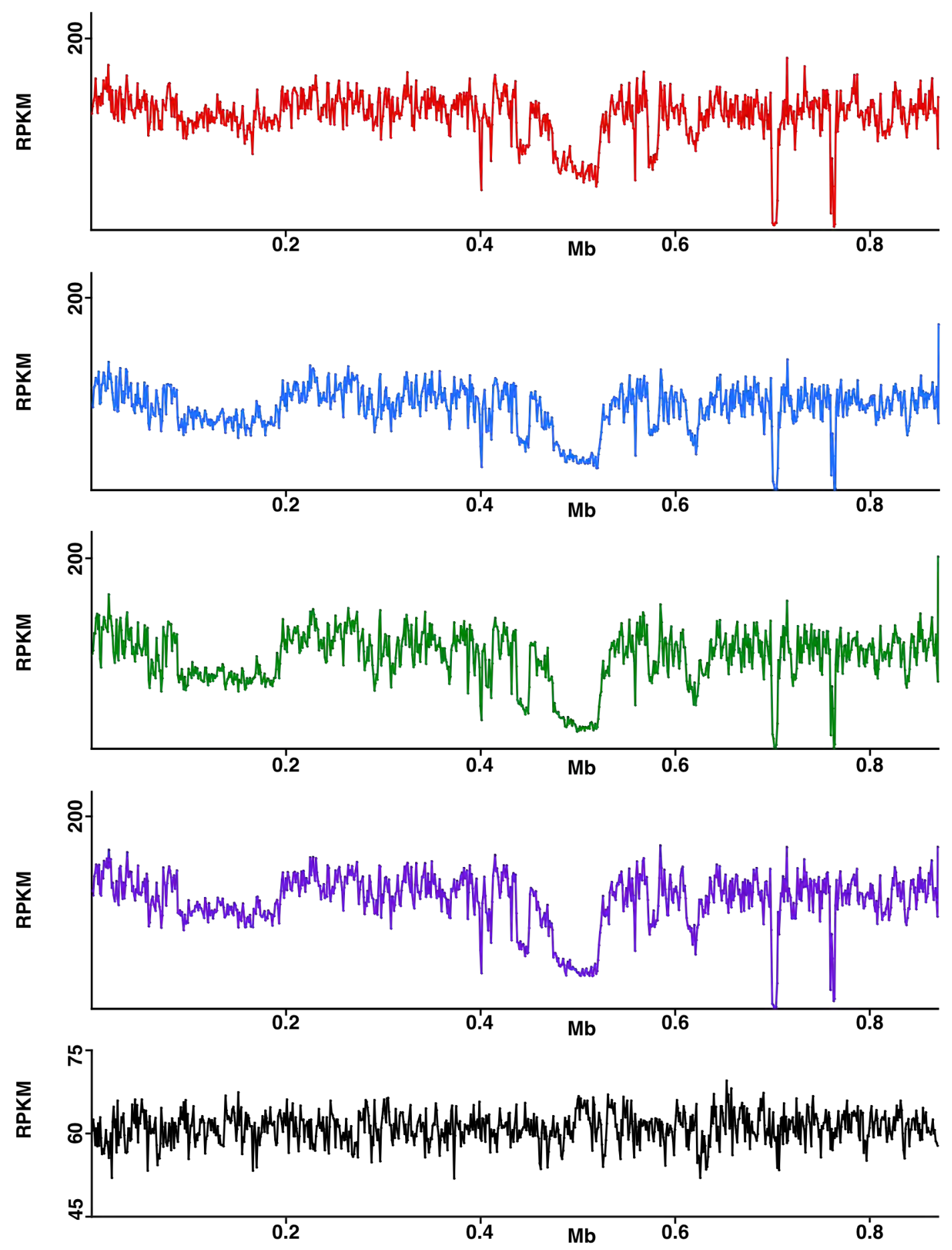

Supplementary Figure 1.S6. Conservation of reference genome plasmid $p R L 12$ in the four plant-selected rhizobial populations. A. Pea. B. Lentil. C. Fava bean. D. Vetch. E. \%GC. RPKM coverage along the reference genome sequence is indicated by a continuous line. 


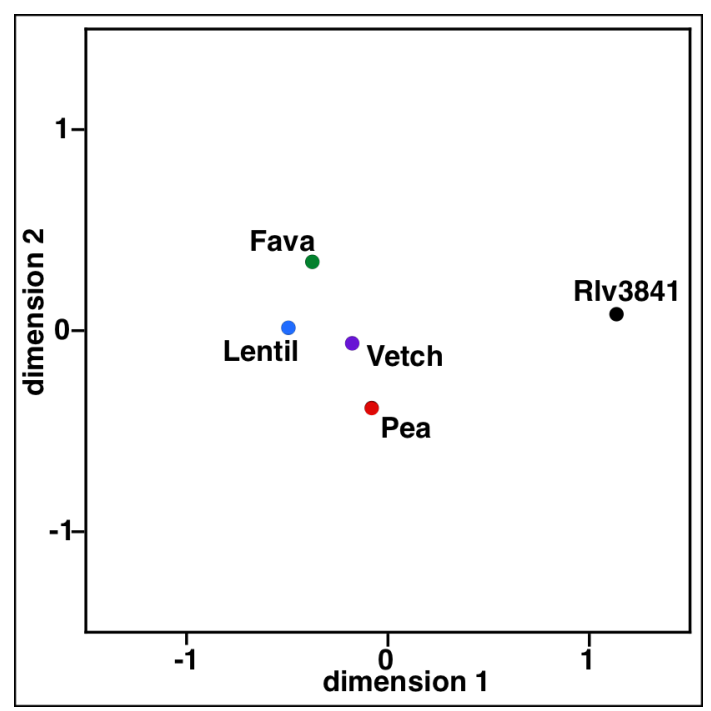

Supplementary Figure 1.S7. Twodimensional representation of genome-wide SNP dissimilarities among plant-selected rhizobial populations and the reference genome. Euclidean distances were calculated from the dissimilarity matrix, and the positions of populations and the reference genome along the two principal coordinates obtained after multi-dimensional scaling were plotted. 

5. CHAPTER 2. Population Genomic Characterization of Native Rhizobium leguminosarum in the Soil 



\subsection{INTRODUCTION}

The specificity of rhizobia-legume symbioses has traditionally allowed the use of most probable number (MPN) techniques to enumerate soil rhizobia that are able to nodulate trap plants (Brockwell, 1963, Vincent, 1970), and numerous studies have been carried out in the past, using Pisum sativum or Vicia spp. as trap plants, in order to estimate the size of symbiotic Rhizobium leguminosarum bv. viciae (RIv) populations in natural and agricultural soils. Magnitudes of 1 to $10^{5}$ RIv have been estimated per $\mathrm{g}$ of soil (Louvrier et al., 1996, Lakzian et al., 2002, Depret et al., 2004, Ballard, 2004, Drew et al., 2012). It is estimated that one gram of soil can contain up to $10^{9}$ microorganisms (Knietsch et al., 2003, Delmont et al., 2011), so Rlv would amount, at most, to $0.01 \%$ of the total soil microbiota. Despite the low probability that a given Rhizobium ends up nodulating its legume (estimated as one in one million; (Denison and Kiers, 2004), these microorganisms can persist for years in soil, even in the absence of their legume (Downie, 2010). Therefore symbiosis is not the only lifestyle of Rlv, in fact it is just an episodic lifestyle. The presence in soil of rhizobia that are unable to form nodules has been inferred repeatedly from the failure of rhizobial inoculants (Moawad and Bohlool, 1984, Streeter, 1994). This non-symbiotic character has been related to the loss of symbiosis plasmids (Leyva et al., 1987). The exchange of symbiotic plasmids among rhizobia with different genomic backgrounds has been described (Brewin et al., 1980), including transfer to non-symbiotic rhizobia (Sullivan et al., 1995, Abe et al., 1998).

During the last decade numerous new Rhizobium species have been described; noteworthy among them is a high number of non-symbiotic species. These new, nonsymbiotic rhizobia were isolated from legume rhizospheres, like $R$. pusense from Cicer arietinum (Panday et al., 2011) or R. alamii from Medicago ruthenica or Phaseolus vulgaris; furthermore $R$. alamii was also found in the rhizosphere of non-legumes plants like Arabidopsis thaliana or Hedysarum coronarium (Berge et al., 2009). The presence of non-symbiotic rhizobia has been described as widespread among other plants like Oryza sativa (Zhang et al., 2011) or Populus spp. (García-Fraile et al., 2007). All these bacteria appear to lack genes related with symbiosis and nitrogen fixation, but among their large genome they posses other different capabilities, such as plant growth promotion (Bresson et al., 2013, Spaepen et al., 2014), stress protection (Bhattacharjee et al., 2011, Tittabutr et al., 2013, Kong et al., 2015) or disease suppression (Arfaoui et al., 2006, Akhtar and Siddiqui, 2008, Kawaguchi, 2014).These reported positive effects on plant growth (legumes and non-legumes alike) point to 
potential roles of the non-symbiotic rhizobia population in soil and to the necessity to access these populations in order to further investigate these roles.

In order to study the entire soil $R$. leguminosarum $(R L)$ population it is necessary to avoid the symbiosis-specific trap plant method. In principle, two approaches could be followed: culture-dependent and culture-independent methods. Present day cultureindependent methods favour metagenomic methodologies. Despite its attractiveness, metagenomics has limited usefulness for rhizobia; Since Rlv is a minor component of the soil microflora, sequencing depths of up to $1 \mathrm{~Tb}$ (largest metagenomics datasets) would be needed in order to obtain $100 \mathrm{Mb}$ of Rlv sequence. This amounts to a $15 \mathrm{x}$ coverage of one single genome, and would be insufficient to study a whole RIv population (Jorrín and Imperial, 2015a). Other culture-independent methods, such as direct gene amplification from soil DNA (Zeze et al., 2001, Sarita et al., 2005, Ando et al., 2005), necessarily restrict the analysis to the number of chosen markers and limits any analysis of the entire RL soil population. This leaves culture-dependent methods.

Different selective medium have been developed to isolate rhizobia directly from soil, based on antimicrobial substances (Graham, 1969, Pattison and Skinner, 1974, Barber, 1979, Soberón-Chávez and Nájera, 1989, Segovia et al., 1991, Laguerre et al., 1993a, Bromfield et al., 1994, Louvrier et al., 1995) and metal resistances (Gault and Schwinghamer, 1993, Tong and Sadowsky, 1994, Kinkle et al., 1994), but a limited number of studies have been devoted to the investigation of native soil RL without recourse to trap plants. All of them combine a semi-selective media followed by some sort of screening, and have focused their efforts on different RL biovars (bv.), such as bv. trifolii (Jarvis et al., 1989), bv. phaseoli (Segovia et al., 1991) and bv. viciae (Laguerre et al., 1993a, Louvrier et al., 1995, Laguerre et al., 2003), as well as on nonsymbiotic strains (Soberón-Chávez and Nájera, 1989). However, none of these studies aimed at the entire indigenous soil RL population without restriction. In addition to RL, other rhizobia have been isolated directly from soil without recourse to trap plants: Ensifer meliloti (Bromfield et al., 1995, Hartmann et al., 1998) and Mesorhizobium loti (Sullivan et al., 1996). A recent study reports the isolation of Rhizobiaceae-related species from soil and root surface of Phaseolus vulgaris and Acacia spp. (MirandaSánchez et al., 2015). These cultivation-dependent methods use the inability of RL to grow on LB as a selective culture-dependent step, which reduces the number of colonies that need to be screened, as well as different combinations of molecular screening methods (probe hybridization, total DNA RFLP, plasmid restriction profile or PCR-RFLP). 
Several studies used $16 \mathrm{~S}$ rRNA as a gene marker to describe RL diversity. 16S rRNA allows reconstruction of the phylogenetic relationship among species, and was employed the first time as marker in 1977 (Fox et al., 1977). Since then, it has been used for bacterial identification and classification, as well as for amplicon metagenomic studies (Rådström et al., 1994, Parente et al., 2016). Certain bacterial groups like Bradyrhizobiaceae, Bosea or Afipia showed low intrinsic $16 \mathrm{~S}$ variability, giving this marker insufficient resolving power to describe variability in these groups (Khamis et al., 2005, Adékambi et al., 2009). In the search for alternative markers, rpoB has appeared as a substitute. The $r p o B$ gene codes for the beta subunit of RNA polymerase, and as 16S rRNA, it is a universal (Case et al., 2007), present in Bacteria and Archaea (Pühler et al., 1989). Generalized sequencing of bacterial genomes shows that 16S rRNA genes are often present in several copies (Ellwood and Nomura, 1980, Loughney et al., 1983, Rainey et al., 1996). Usually, but not always, these are identical copies, but in some cases, these copies differ, thus invalidating its use as phylogenetical marker (Klappenbach et al., 2001, Větrovský and Baldrian, 2013). Phylogenies derived from $r p o B$ are in better agreement with DNA-DNA hybridization results than 16S phylogenies (Korczak et al., 2004, Adékambi et al., 2008), which suggests that $r p o B$ is a more powerful marker than 16S rRNA (Morse et al., 1996). Likewise, rpoB possesses hypervariable regions flanked by conserved ones (Khamis et al., 2003), allowing primer design in these conserved areas in order to sequence a shorter but equivalent variable fragment to reduce the genotyping costs (Case et al., 2007). The $r p o B$ marker has been used for numerous groups such as Enterobacteriaceae (Mollet et al., 1997), Brucella (Marianelli et al., 2006) or Frankia (Bernèche-D'Amours et al., 2011). Furthermore, rpoB has been tested as a substitute for amplicon metagenomics with excellent results. However, despite the fact that it was able to detect as much diversity and richness as $16 \mathrm{~S}$ rRNA with ten times less reads (Vos et al., 2012), the insufficient $r p o B$ databases available and the widespread use of 16S rRNA, have limited the use of $r p o B$ for amplicon metagenomics (Case et al., 2007, Vos et al., 2012). In addition to $r p o B, c p n 60$ (60 kDa chaperone; groEL) has been developed as a microbial ecological marker (Links et al., 2012) for human and plant microbiome studies (Oliver et al., 2008, Schellenberg et al., 2011, Chaban et al., 2014, Links et al., 2014). Cpn60 is a better marker than 16S rRNA in samples with high host DNA content; however this gene is often duplicated (Johnson et al., 2015) and sometimes more non-identical copies are present in the genome (Gould et al., 2007). 
In this study we set out to characterize an indigenous Rhizobium leguminosarum soil population avoiding plant selection, in order to compare it with previously characterized host-selected subpopulations from this soil (Jorrín and Imperial, 2015b). The rationale for this approach has been already presented (Jorrín and Imperial, 2015b; Chapter 1 of this Thesis) and derives directly from results presented in the previous chapter: if legume plants can select specific genotypes from those available in the soil, what does this represent in terms of population diversity? And, are selected genotypes chosen among the most abundant, or are they chosen even though they may be underrepresented in soil? 


\subsection{RESULTS}

\subsubsection{Soil-selected population}

The soil-selected population consisted of 118 strains obtained after direct soil cultivation and isolated using a semi-selective medium (Louvrier et al., 1995), followed by PCR-based screening of chromosomal (fnrN and $g / n I l$ ) and symbiotic markers (nodC and nifH). A total of $130 \mathrm{~g}$ of soil were processed in thirteen different independent isolation trials, and Table 2.1 presents a summary of results. A total of $5.98( \pm 3.28) \cdot 10^{6}$ colony formation unit (cfu) aerobic bacteria $\cdot \mathrm{g}^{-1}$ soil were estimated for

Table 2.1. Amount of different soil bacteria isolated from $\mathrm{P} 1 \mathrm{Dijon}$ soil.

\begin{tabular}{lc}
\hline & \multicolumn{1}{c}{${\mathrm{cfu} \cdot \mathrm{g}^{-1}}^{-1}$} \\
\hline Total aerobic & $5.98 \cdot 10^{6} \pm 3.28 \cdot 10^{6}$ \\
Total Gram - & $4.62 \cdot 10^{5} \pm 1.16 \cdot 10^{5}$ \\
Rhizobia & $2.00 \cdot 10^{5} \pm 8.53 \cdot 10^{4}$ \\
\hline Calculated as the media of thirteen independent \\
isolates
\end{tabular}

P1 Dijon soil with TS medium. Soil was also directly plated on MNBP medium (semiselective medium); this inhibits growth of Gram-positive bacteria due to the presence of penicillin $G$ and bacitracin, as well as that of fungi and actinomycetes because of the existence of cycloheximide, benomyl and PCNB. The estimated Gram-negative bacterial population in P1 Dijon soil was $4.62( \pm 1.16) \cdot 10^{5} \mathrm{cfu} \cdot \mathrm{g}^{-1}$, one order of magnitude lower than the total aerobic estimated. After two consecutive screening steps for negative growth on LB, the final count of bacteria (putative rhizobia) was $2 \cdot 10^{5}\left( \pm 8.53 \cdot 10^{4}\right) \mathrm{cfu} \cdot \mathrm{g}^{-1}$, only a $43 \%$ of the total bacteria that grew on MNBP medium. These strains were screened for chromosomal (fnrN and $g I n I l)$ and symbiotic (nodC and nifH) markers, and Table 2.2 shows the abundance $\left(\mathrm{cfu} \cdot \mathrm{g}^{-1}\right)$ of the rhizobial genotypes obtained. A $23 \%\left(4.64 \cdot 10^{4} \pm 4.18 \cdot 10^{4}\right)$ of the total Rhizobia screened lacked all of the markers analysed (this group was labelled as "Rhizobia other"); $58 \%$ $\left(1.16 \cdot 10^{5} \pm 5.79 \cdot 10^{4}\right)$ of the screened strains were positive for glnll but not for fnrN (Rhizobia $\mathrm{gln} / \mathrm{l}+$ ) and $19 \%\left(3.76 \cdot 10^{4} \pm 1.94 \cdot 10^{4}\right)$ of the total strains screened were

Table 2.2. Amount of different rhizobial genotypes as result of the markers screening.

\begin{tabular}{lccccc}
\hline & fnrN & glnll & nodC & nifH & $\mathrm{cfu} \cdot \mathrm{g}^{-1}$ \\
\hline Rhizobia other & - & - & $+/-$ & $+/-$ & $4.64 \cdot 10^{4} \pm 4.18 \cdot 10^{4}$ \\
Rhizobia glnll + & - & + & $+/-$ & $+/-$ & $1.16 \cdot 10^{5} \pm 5.79 \cdot 10^{4}$ \\
Rhizobium sp. total & + & $+/-$ & $+/-$ & $+/-$ & $3.76 \cdot 10^{4} \pm 1.94 \cdot 10^{4}$ \\
Rhizobium sp. symbiotic & + & $+/-$ & + & + & $1.60 \cdot 10^{4} \pm 1.44 \cdot 10^{4}$ \\
Rhizobium sp. non-symbiotic & + & $+/-$ & - & - & $1.77 \cdot 10^{4} \pm 9.20 \cdot 10^{3}$ \\
Rhizobium sp. other & + & $+/-$ & $+/-$ & $+/-$ & $4.39 \cdot 10^{3} \pm 4.85 \cdot 10^{3}$ \\
\hline
\end{tabular}


positive for fnrN. These bacteria were tentatively assigned as Rhizobium sp. (Rhizobium sp. total). Out of the total Rhizobium sp. approximately $42 \%\left(1.60 \cdot 10^{4} \pm\right.$ $1.44 \cdot 10^{4}$ ) were positive for both symbiotic markers (Rhizobium sp. symbiotic) and $46 \%$ $\left(1.77 \cdot 10^{4} \pm 9.20 \cdot 10^{3}\right)$ were negative for both symbiotic markers (Rhizobium sp. nonsymbiotic). Only $12 \%\left(4.39 \cdot 10^{3} \pm 4.85 \cdot 10^{3}\right)$ were positive for only one of the symbiotic markers screened (Rhizobium sp. other).

\subsubsection{Nodulation tests}

In view of the high number of isolates lacking one or both symbiotic markers, the total 118 strains isolated from soil were tested for their ability to nodulate legume roots, and the results are summarized in Table 2.3. All the isolates were first tested with Vicia sativa plants in square plates containing Fahraeus nitrogen-free agar solution. Four weeks after inoculation, nodule formation was recorded in a total of fifty four strains (45.76\%). Isolates that did not form nodules with $V$. sativa were tested with Trifolium repens plants, also in square plates containing Fahraeus nitrogen-free agar solution, and twelve isolates $(10.17 \%)$ did form nodules with $T$. repens. Again, isolates negative with $V$. sativa or $T$. repens were tested with Phaseolus vulgaris, this time in Leonard Jars. No isolates produced nodules in P. vulgaris. Fifty-two strains $(44.07 \%)$ did not form nodules with any of the plant tested. All the strains that formed nodules with one of the legumes tested were positive for both symbiotic markers (52 isolates) or for just one of them (14 isolates). None of the fifty-two isolates that lacked both symbiotic markers were able to elicit nodules with any of the plants tested.

Table 2.3. Cross inoculation assay results

\begin{tabular}{lcccc}
\hline & V. sativa & T. repens & P. vulgaris & Non-symbiotic \\
\hline Rhizobium sp. symbiotic & 44 & 8 & - & - \\
Rhizobium sp. non-symbiotic & - & - & - & 52 \\
Rhizobium sp. other & 10 & 4 & - & - \\
\hline
\end{tabular}

\subsubsection{Strains Genotyping with the rpoB marker}

A fragment of the $r p o B$ gene (447 nt) was amplified and sequenced for the 118 rhizobial soil isolates, a multiple alignment that included database sequences from the order Rhizobiales was constructed, and Neighbor-joining phylogenies were derived from it. The resulting $r p o B$ phylogenetic tree showed that all the soil isolates belong to the order Rhizobiales (Figure 2.1, Figure 2.2); these isolates were clustered in 26 groups (named A through Z). Groups A-D were related with Phyllobacterium species, but not with sequences from the Phyllobacteriaceae family. No rpoB sequence of any 


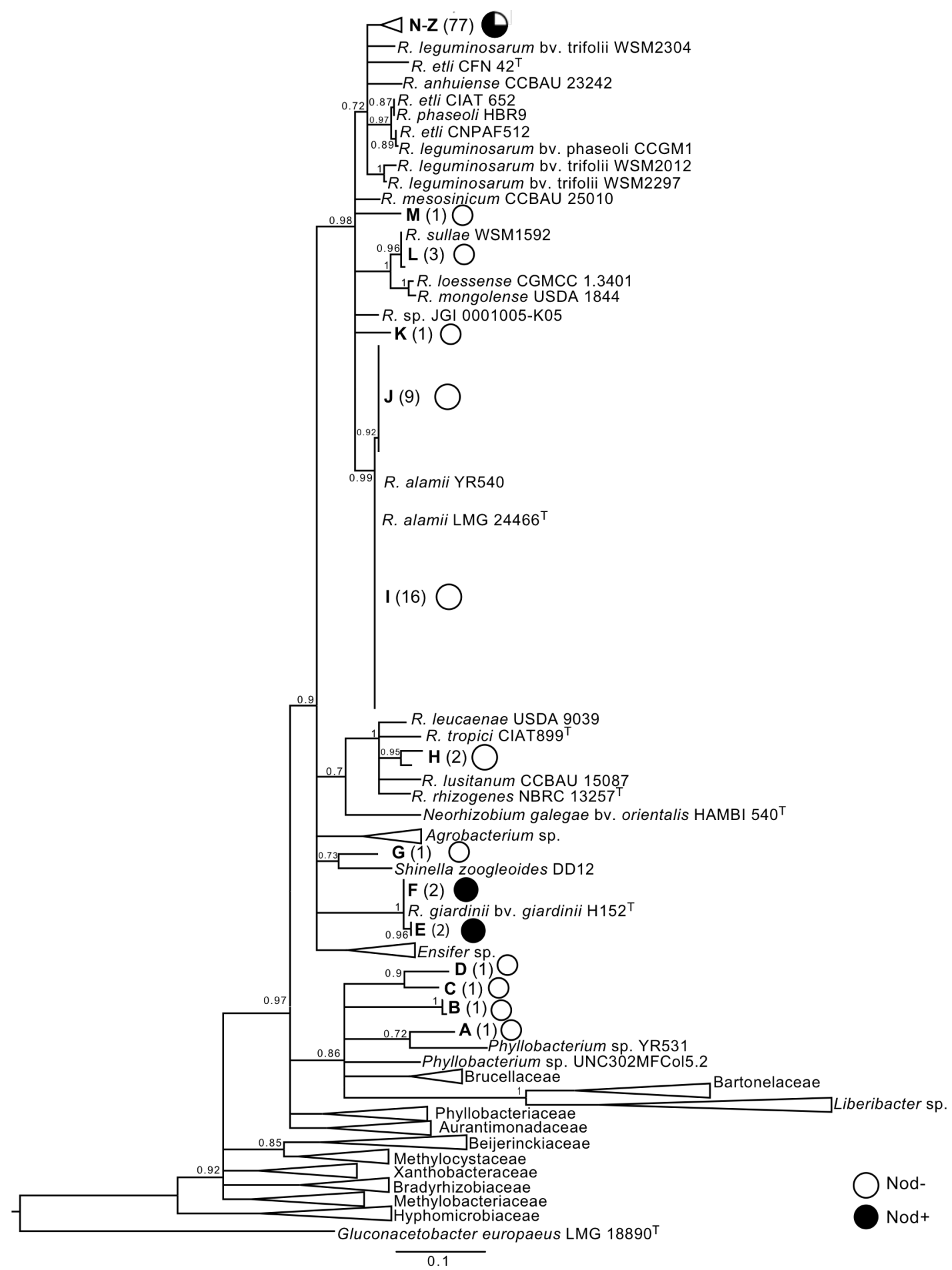

Figure 2.1. Phylogenetic tree based on partial (447 nt) rpoB sequences showing the relationship of P1 soil isolates and related species within the order Rhizobiales. The tree was constructed by the neighbor-joining method. The significance of each branch is indicated by a bootstrap value calculated for 1,000 subsets. Soil isolates were grouped by similarity and are shown as a capital letter followed by the number of isolates that the group contains. Circles indicate fraction of symbiotic (filled) and non-symbiotic isolates in a given group. Nodes with bootstrap values lower than 0.7 were collapsed. Bar represents $10 \%$ substitution. The rpoB sequence of Gluconacetobacter europaeus LMG $18890^{\top}$ was used as outgroup. Abbreviations: R. Rhizobium. 


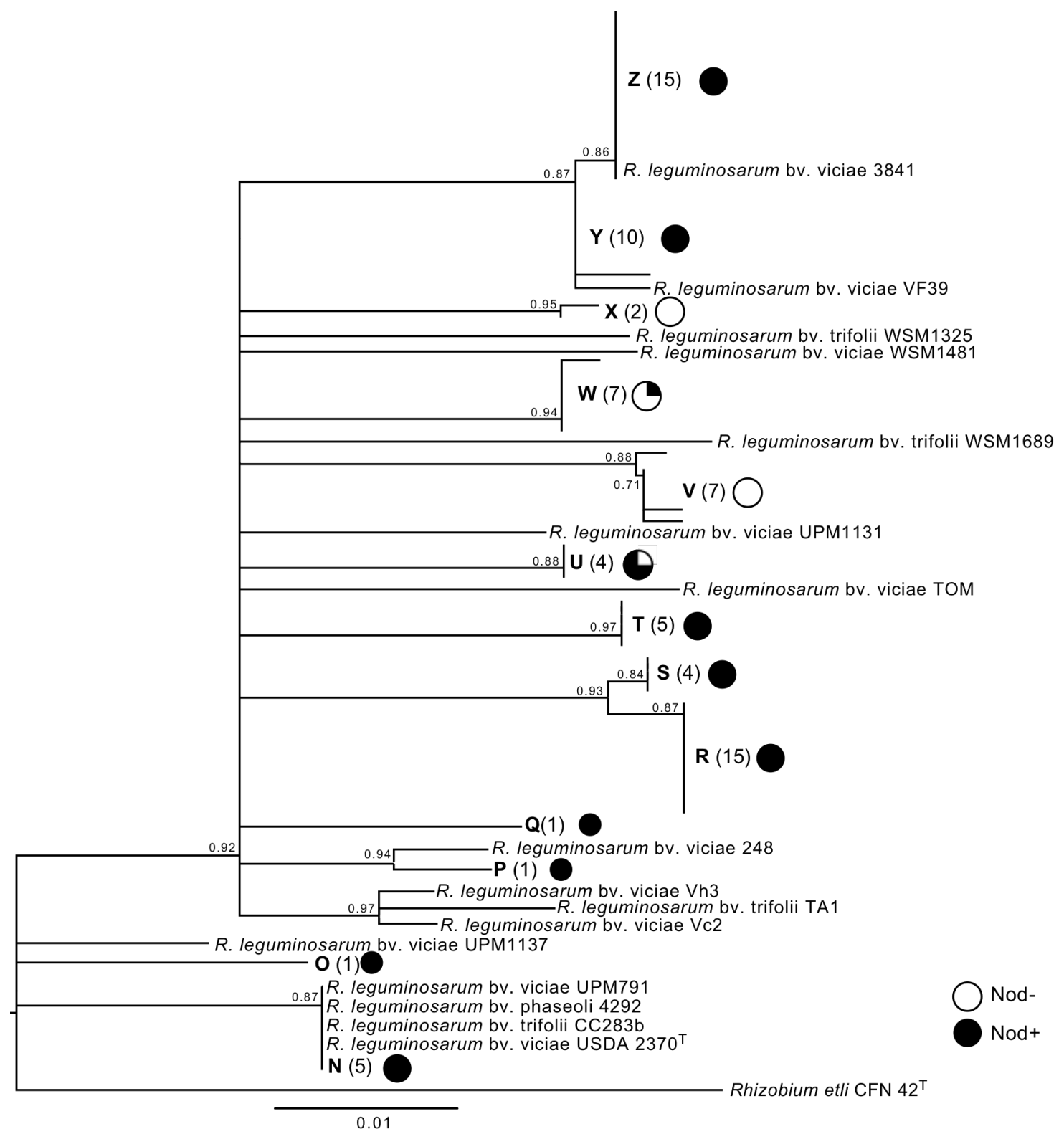

Figure 2.2. Phylogenetic tree based on partial ( $447 \mathrm{nt}) r p o B$ sequences showing the relationship of a subsample of $\mathrm{P} 1$ soil isolates (N-Z; see Fig. 2.1) and related species within the genus Rhizobium. The tree was constructed by the neighbor-joining method. The significance of each branch is indicated by a bootstrap value calculated for 1,000 subsets. Soil isolates were grouped by similarity and are shown as a capital letter followed by the number of isolates that the group contains. Circles indicate fraction of symbiotic (filled) and non-symbiotic isolates in a given group. Nodes with bootstrap values lower than 0.7 were collapsed. Bar represents $1 \%$ substitution. The rpoB sequence of Rhizobium etli CFN $42^{\top}$ was used as outgroup. Abbreviations: R. Rhizobium. 
Phyllobacterium type strain is available in databases, but a complete $16 \mathrm{~S}$ tree of all the type strains from species of the Rhizobiales (data not shown) showed that many of the sequences named as Phyllobacterium spp. were not related with other members of the family Phyllobacteriaceae, such as Mesorhizobium, Hoeflea, Nitrareductor or Aminobacter species. The 114 remaining strains clustered within the family Rhizobiaceae. Two groups were related with Rhizobium giardinii bv. giardinii $\mathrm{H}^{152}{ }^{\mathrm{T}}$ : group $E$ (symbiotic with $V$. sativa) and group $F$ (symbiotic with $T$. repens). A nonsymbiotic strain (group G) was related to Shinella zoogeloides DD12 and two other non-symbiotic strains (group $\mathrm{H}$ ) were related with $R$. rhizogenes NBRC $13257^{\top}, R$ lusitanum CCBAU 15087, R. tropici CIAT899 ${ }^{\top}$ and $R$. leucaenae USDA 9039. Two large non-symbiotic groups ( $\mathrm{I}$ and $\mathrm{J}$ ) were related to $R$. alamii strains. Groups $\mathrm{K}$ and $\mathrm{M}$ appeared to be Rhizobiaceae-related species, but no direct phylogenetic relationship was found with strains present in the tree. Group $L$ was composed of three nonsymbiotic strains related to $R$. sullae WSM1592 (Figure 2.1). The remaining 77 isolates appeared to be related to $R$. leguminosarum and cluster in groups $\mathrm{N}-\mathrm{Z}$. They were plotted in a sub-tree (Figure 2.2). Five symbiotic strains formed group $\mathrm{N}$ and were related with RIv USDA 2370 type strain, RIv UPM791, RIt CC283b and RIp 4292, two of them were effective with $V$. sativa and three with $T$. repens. Group $O$ was formed by a single $T$. repens symbiotic strain. Group P, closely related to RIv 248 was symbiotic with $V$. sativa. All strains forming groups $Q, R, S$ and $T$ were symbiotic with $V$. sativa (ie. belong to biovar viciae). Group $U$ was formed by three bv. viciae strains and one non-symbiotic strain. Exclusively non-symbiotic rhizobia constituted groups $\mathrm{V}$ and $\mathrm{X}$, whereas group $\mathrm{W}$ was composed of five non-symbiotic isolates and two $V$. sativa symbiotic strains. Group Y, related to RIV VF39, contained V. sativa symbiotic bacteria, while group $Z$ consisted of nine $V$. sativa symbiotic bacteria, six $T$. repens symbiotic strains. Supplementary Table 2.S1 summarizes all the information.

16S rRNA sequences were obtained for 36 representative strains from the 26 different groups defined above, in order to gauge the accuracy of $r p o B$ as phylogenetic marker; two phylogenetic trees were constructed with the same strains (Supplementary Figure 2.S1, rpoB (A) and 16S rRNA (B)). Partial rpoB gene sequences were able to resolve more with higher resolution phylogenetic relationships among the strains used to construct in this study, even aligning a shorter sequence (504nt) than that used for 16S rRNA $(1,287 \mathrm{nt})$. rpoB grouped together all the species that belong to the family Rhizobiaceae, while 16S rRNA formed different groups for Agrobacterium, Ensifer and Neorhizobium species. 16S rRNA did not relate strains S07001, S11101, S3B049 and S07051 with Ensifer clade. The main difference in the architecture of both trees was the localization of the $R$. tropici CIAT899 ${ }^{\top}$ group: in the $16 \mathrm{~S}$ rRNA tree this group was 
associated to $R$. leguminosarum, while in the rpoB tree it appeared at the same level as Ensifer, Agrobacterium and Neorhizobium clades. rpoB was also capable to differentiate highly related strains as it was the case for the Rhizobium leguminosarum group, where $r p o B$ differentiated fifteen different groups, while 16S rRNA resolved only four.

\subsubsection{Next generation sequencing of the pooled soil rhizobial population}

DNA from pooled samples of the native soil rhizobial population was submitted to next-generation sequencing (Pool-Seq) so that, on average, each of the genomes was sequenced to 1 to $2 x$ coverage. A Pool-Seq sample formed by the 118 strains isolated directly from soil was sequenced (Illumina HiSeq2000, 180 bp PE libraries, 100 bp reads, 12 Mreads; BGI Hong Kong). Individual sequencing reads from the soil population were recruited to the reference genome (Materials and Methods, (Jorrín and Imperial, 2015b) and coverage results were normalized to correct for minor differences in sequencing depth among different Pool-Seq datasets.

\subsubsection{Presence of sequences from the $R$. leguminosarum bv. viciae 3841 reference} genome in the soil Pool-Seq dataset

The $R$. leguminosarum bv. viciae 3841 reference genome is multipartite and contains six large plasmids : pRL7 (151.5 kb) through pRL12 (870 kb), in addition to a $5,057 \mathrm{~kb}$ circular chromosome (Young et al., 2006). The presence and conservation of sequences from these seven replicons in the Pool-Seq dataset from P1 soil was investigated (Table 2.4). Sequences from the two smaller plasmids, pRL7 and pRL8 were minor components of the Pool-Seq soil population, suggesting that these plasmids, if present, are a minor component of the P1 soil rhizobial population (Jorrín

Table 2.4. Average coverage (RPKM) of each reference genome (Rhizobium leguminosarum bv. viciae 3841 ) replicon by soil Pool-Seq sample datasets.

\begin{tabular}{cc}
\hline & Soil \\
\hline Chromosome & 71.1 \\
pRL7 & 5.2 \\
pRL8 & 3.1 \\
pRL9 & 60.8 \\
pRL10 & 46.5 \\
pRL11 & 55.5 \\
pRL12 & 65.1 \\
\hline
\end{tabular}


and Imperial, 2015b). The highest coverage was found for the chromosome $(71.1$ RPKM), followed by the larger plasmids pRL12, pRL9, pRL11 and pRL10 (65.1, 60.8, 55.5 and 46.5 RPKM respectively). All the coverage values for this soil sample were lower in all the cases than those found for plant-selected Pool-Seqs (Table 1.1 from Chapter 1), suggesting that, as expected, the soil population is more dissimilar regarding the reference genome.

Plots of coverage (RPKM) along replicon sequences are presented in Figure 2.3. No overrepresented sequence peaks were found in the Soil Pool-Seq. Conversely; seven regions from the reference genome were absent or near absent in the P1 soil dataset. In addition, plasmids pRL8 and pRL7 were nearly absent, with very few reads being recruited to them. The seven low-coverage chromosomal regions were the same as those found in the host Pool-Seq subpopulations: region 1 (RL0791- RL0841), region 2 (RL1869-RL1944), region 3 (RL2105-RL2195) and region 4 (RL3912RL3956), spanning 51, 76, 91, and 45 genes, respectively. Region 5 spanned 40 genes (pRL120440-pRL120479), region 6 contains 44 genes (pRL110145-pRL110188) and region 7 is present in the symbiotic plasmid pRL10, and spans 128 genes (pRL100011-pRL100138) (Jorrín and Imperial, 2015b).

Despite conservation of the reference genome within the $\mathrm{P} 1$ soil population, not all sequence reads from the Pool-Seq were recruited by the reference genome and, in fact, the unrecruited sequences unique to the $\mathrm{P} 1$ soil datasets constitute a large fraction of the reads, up to $50.0 \%$. Aside from possible artifactual sequences, these sequence reads probably arise from genomic regions present in the $\mathrm{P} 1$ soil population, but absent in the reference genome.

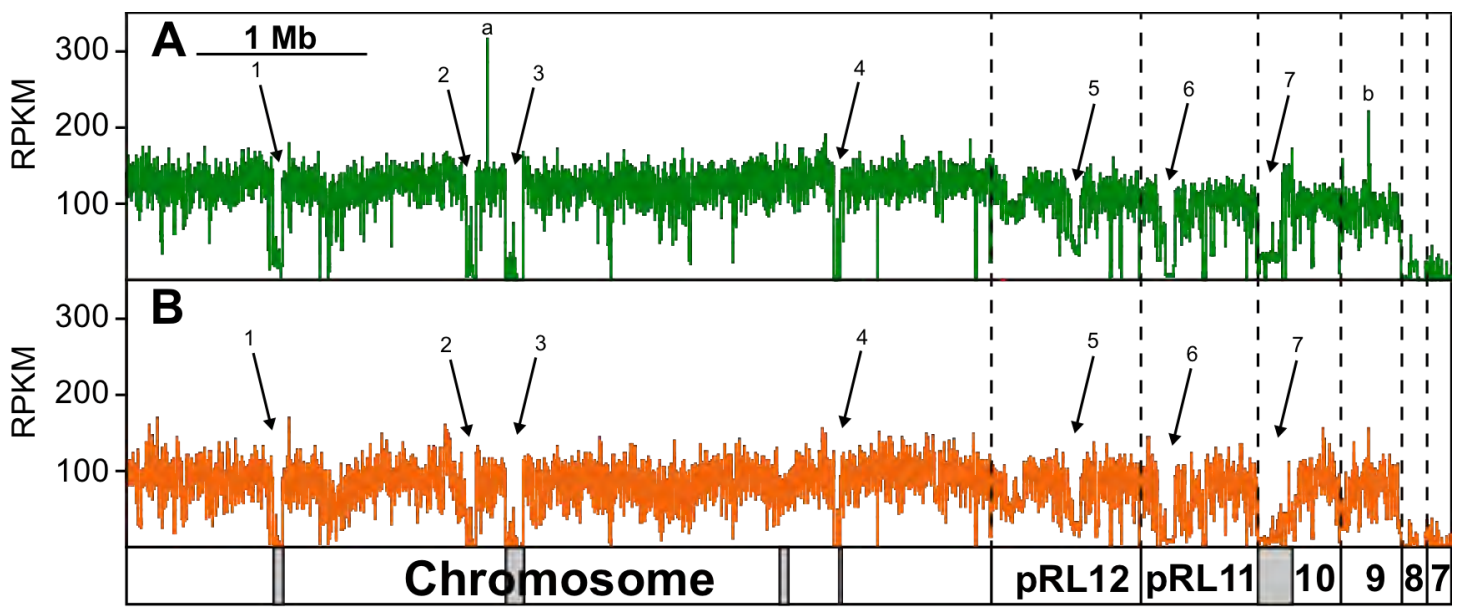

Figure 2.3. Conservation of the $R$. leguminosarum bv. viciae 3841 reference genome in the P1 soil rhizobial population. Coverage (RPKM) of genome regions by Bowtie2 recruited reads from host (A) and soil (B). Sequences of the seven reference replicons were concatenated and are shown along the horizontal axis. The location and span of five low $\mathrm{G}+\mathrm{C}$ islands described by (Young et al., 2006) in the reference genome are indicated by grey bars. Major under-represented and over-represented regions in the P1 soil dataset are indicated by numbers and lowercase letters, respectively. 


\subsubsection{Differential abundance of $R$. leguminosarum bv. viciae 3841 reference genome} sequences in soil Pool-Seq and host Pool-Seqs.

Due to the absence of clearly overrepresented peaks in soil Pool-Seq, a comparison coverage analysis was performed between the host and soil Pool-Seqs, in order to investigate differential gene presence between them. For each gene predicted in the reference genome $R$. leguminosarum bv. viciae $3841, \log _{2}$ of the fold-change comparing soil and host RPKMs was calculated. Genes with coverage lower than 20 RPKM, were considered as absent, and genes with $\log _{2}$ fold-change greater than 2 , or lower than -2, were considered to be more abundant in soil or host Pool-Seqs, respectively. A total of 88 genes were found to be differentially present in soil and host samples (Figure 2.4). Only two genes were more abundant in soil Pool-Seq compared with host Pool-Seqs: pRL120647 (putative transposase-related protein) and pRL120700 (conserved hypothetical exported protein). The remaining 86 genes were more abundant in host-selected populations than in the soil population. Many of them were annotated as hypothetical proteins (RL0067, RL0387, RL0541, RL1056-7, RL1150-1, RL2265, RL2306, RL2676-78, RL2685, RL2958, RL3103, RL3606, pRL100150, pRL100154, pRL100157, pRL100174, pRL100192-4, pRL100216, pRL110110, pRL110118, pRL110120-1, pRL110131, pRL110251-3, pRL110317A, pRL11026, pRL120564 and pRL120568). Other annotations were as follows: no description ( $p R L 100143$ and pRL110197), transmembrane proteins (RL1152, RL2876, RL3101, pRL110116 and pRL110122), different transcriptional regulators (RL2972, RL3102, RL3566, pRL12561A, pRL110105, pRL110119 and pRL110231), genes related to transposases (RL2002, RL2675, pRL110580 and pRL110607), genes related to plasmid mobilization (pRL100215, pRL100219, $p R L 110275$ and $p R L 110276)$, ArdC antirestriction protein (pRL110270 and pRL120563), arginyl-tRNA synthase (RL1058), hydrolase (RL1145), arylsulfatase (RL1149), a cobalt transport cluster (pRL110123-27), pRL12561 (putative glucose-6-phosphate dehydrogenase), the cluster pRL110094-104, that includes a type I restriction enzyme cluster in addition to a transmembrane protein and hypothetical proteins, and genes related with symbiosis, such as $f d x B$ (pRL100156), rhiR and rhiA (pRL100164 and pRL100169), nodN and nodl (pRL100179 and pRL100188) and nifB (pRL100195). 


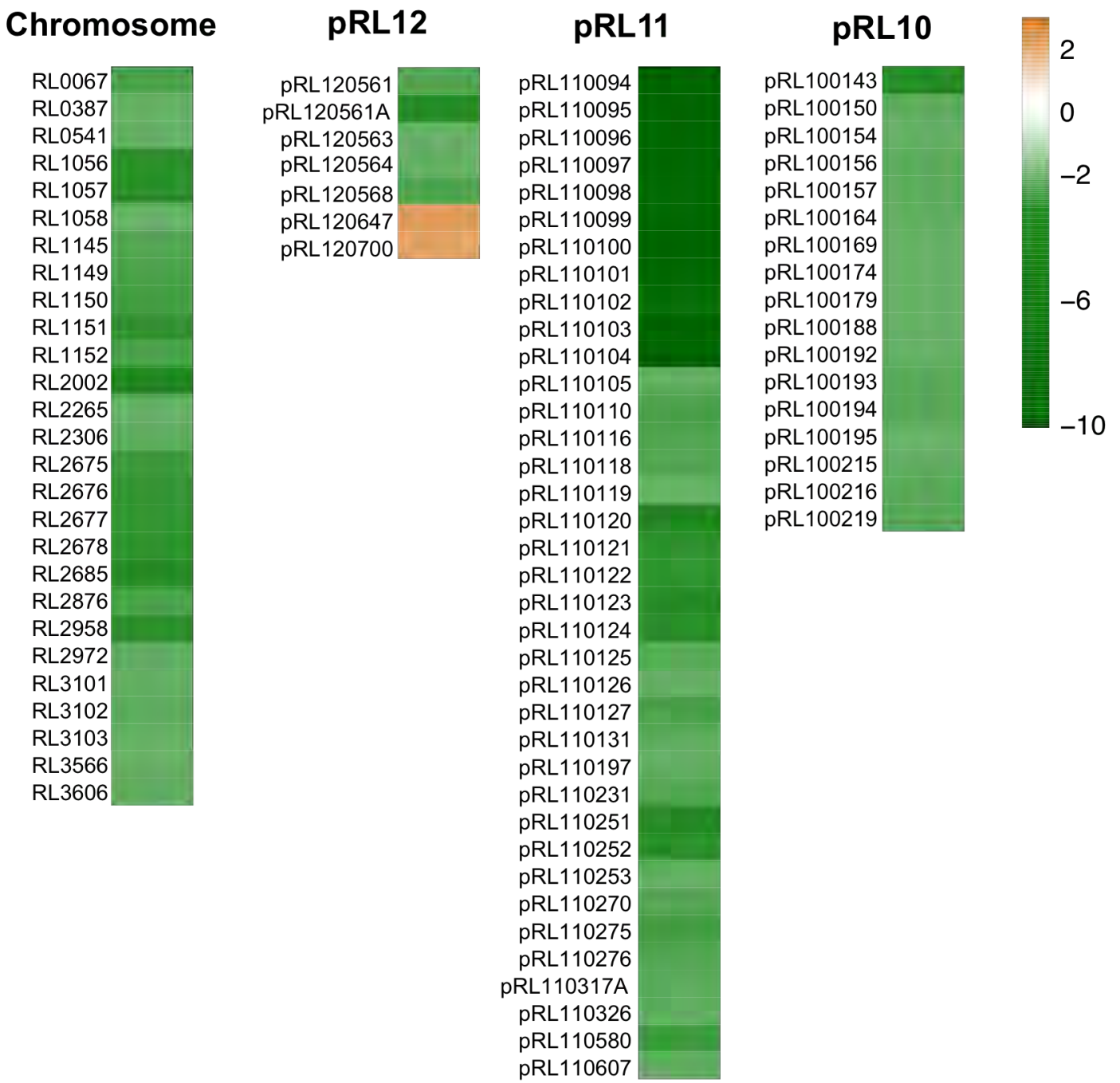

Figure 2.4. Heat map representation of gene conservation in host and soil Pool-Seqs along the four larger replicons in the reference genome. Differential $\log _{2}$ fold-change was calculated for each gene. $\log _{2}$ fold-change greater than 2 (orange) were considered more abundant in soil Pool-Seq, while $\log _{2}$ fold-change lower than -2 (green) were considered to be more abundant in host Pool-Seq.

\subsubsection{Genome-wide sequence polymorphisms in Pool-Seq datasets}

A comparative analysis of significant nucleotide polymorphisms in sequences recruited by the reference genome was carried out. In order to determine if the host subpopulations studied in Chapter 1 (pea, lentil, fava bean and vetch) were significantly different to the soil population, $F_{\mathrm{ST}}$ values were calculated for each SNP in pairwise comparisons between each host and the soil population. SNPs were considered highly significantly different if the $F_{\mathrm{ST}}$ value was greater than 0.25 (Significantly Different Single Nucleotide Polymorphism, SD-SNP). Table 2.5 summarizes the results obtained. Large differences in total numbers of SD-SNPs were found among subpopulations, when compared with soil Pool-Seq. Pea subpopulations showed the highest differentiation with soil population, with a total number of 97,949 
SD-SNPs, followed by vetch (10,745 SD-SNPs), lentil (6,341 SD-SNPs) and fava bean (5,239 SD-SNPs). This represented over an order of magnitude higher SD-SNPS $(1.264 \%$ of total sites) in peas than in the remaining subpopulations $(0.068-0.139 \%$ of total sites). This tendency was maintained throughout all the replicons, with the pea subpopulation always showing an order of magnitude higher SD-SNPs (1.140-1.498\% of all sites per replicon), except for plasmids $p R L 7$ and $p R L 8$. In replicon to replicon comparisons, the lentil subpopulation exhibited an $2.5 \times$ increase in SD-SNPs for plasmid pRL10 $(0.197 \%)$ as compared to global abundance $(0.082 \%)$, which suggests that, compared to the soil population, the symbiotic plasmid recruited more dissimilar reads than the global genome. A similar situation was found in fava bean where plasmids pRL12 (0.159 \% SD-SNPs) and pRL10 (0.223 \%SD-SNPs) also showed an increase with respect to global SD-SNPs $(0.068 \%)$. On the contrary, plasmid pRL9 presented reduced SD-SNPs in lentil (from 0.082 to $0.024 \%$ ), fava bean (from 0.068 to $0.011 \%$ ) and vetch (from 0.139 to 0.044 ) subpopulations, suggesting that plasmid pRL9 is more conserved in these three subpopulations than the global genome.

Table 2.5. Genome-wide abundance of significantly different single nucleotide polymorphisms in Pool-Seq DNA samples respect to soil Pool-Seq ${ }^{a}$

\begin{tabular}{lcccc}
\hline & Pea & Lentil & Fava bean & Vetch \\
\hline Total & 97,949 & 6,341 & 5,239 & 10,745 \\
Total \% & 1.264 & 0.082 & 0.068 & 0.139 \\
Chromosome \% & 1.291 & 0.057 & 0.036 & 0.117 \\
pRL12 \% & 1.421 & 0.148 & 0.159 & 0.250 \\
pRL11 \% & 1.498 & 0.142 & 0.112 & 0.191 \\
pRL10 \% & 1.140 & 0.197 & 0.223 & 0.225 \\
pRL9 \% & 1.191 & 0.024 & 0.011 & 0.044 \\
pRL8 \% & 0.077 & 0.004 & 0.007 & 0.004 \\
pRL7 \% & 0.126 & 0.092 & 0.088 & 0.057 \\
\hline
\end{tabular}

${ }^{a}$ Expressed as total number and as percentages of each replicon and of the total reference genome sequenced. 


\subsubsection{Sequence polymorphisms in specific regions of Pool-Seq datasets}

Two contrasting regions have been selected to study the profile of SD-SNPs of host subpopulations (pea, lentil, fava bean and vetch) respect to $\mathrm{P} 1$ soil population: 16S rRNA-23S rRNA region, encoding conserved housekeeping rRNAs, and nod cluster region, responsible for the synthesis of the Nod factor, the main symbiotic signal.

\subsubsection{16S rRNA-23S rRNA region}

The reference genome $R$. leguminosarum bv. viciae 3841 contains three copies of the rRNA operon, all identical and located in the chromosome, with the conserved structure: 16S rRNA -lle tRNA -Ala tRNA -23S rRNA -5S rRNA (Young et al., 2006). Pool-Seq datasets were recruited to this region; coverage and $F_{S T}$ pairwise comparisons against soil population were plotted along the region (Figure 2.5). The high level of coverage in the soil Pool-Seq (above 800 RPKM for 165 and 23S) was, nonetheless, lower than that found in host subpopulations (above 1,000 RPKM), which probably reflects the soil population composition, where not all the strains are Rhizobium spp. Coverage decreased drastically in the 16S-23S intergenic region, suggesting that this part of the region may not be conserved, or present, in rhizobia from the P1 soil population (Figure 2.5.E). This was also true for the lle- and Ala-tRNAs present in the 16S-23S intergenic region of the reference strain. It is worth noting that decreases in sequence coverage also occurred at the 5 '-end of the 235 rRNA and for $5 S$ rRNA in the soil Pool-Seq population.

A few SD-SNPs were found in this region when comparing with the soil Pool-Seq, five for pea Pool-Seq, four for lentil, and three for fava bean and vetch Pool-Seqs (Table 2.6). Two of these SD-SNPs were present in the four host subpopulations, and are localized in the intergenic region between 16S and Ile-tRNA (205 and 251 bp downstream the end of $16 \mathrm{~S}$ ). These values reflected a significant differentiation between each host subpopulation compared with soil population, where the main nucleotide in host subpopulations differed from the most abundant one in soil population (T/C and T/G for host/soil populations) (Table 2.7). Another SD-SNP (G/A for host/soil) also localized in the intergenic region between 16S-Ile-tRNA, and was only significantly different for pea and lentil subpopulations as compared with soil PoolSeq. In fava bean and vetch Pool-Seqs the G nucleotide frequency was also high $(0.88$ and 0.87 respectively) but $F_{\mathrm{ST}}$ values compared with soil were not greater than 0.25 (0.1967 and 0.1801 , respectively). Two SD-SNPs were exclusive for pea subpopulation. In one of them, localized in the $16 \mathrm{~S}$ gene (position 1,069), $82 \%$ of the pea reads recruited by the rRNA region presented a $T$, while in soil population $72 \%$ of 

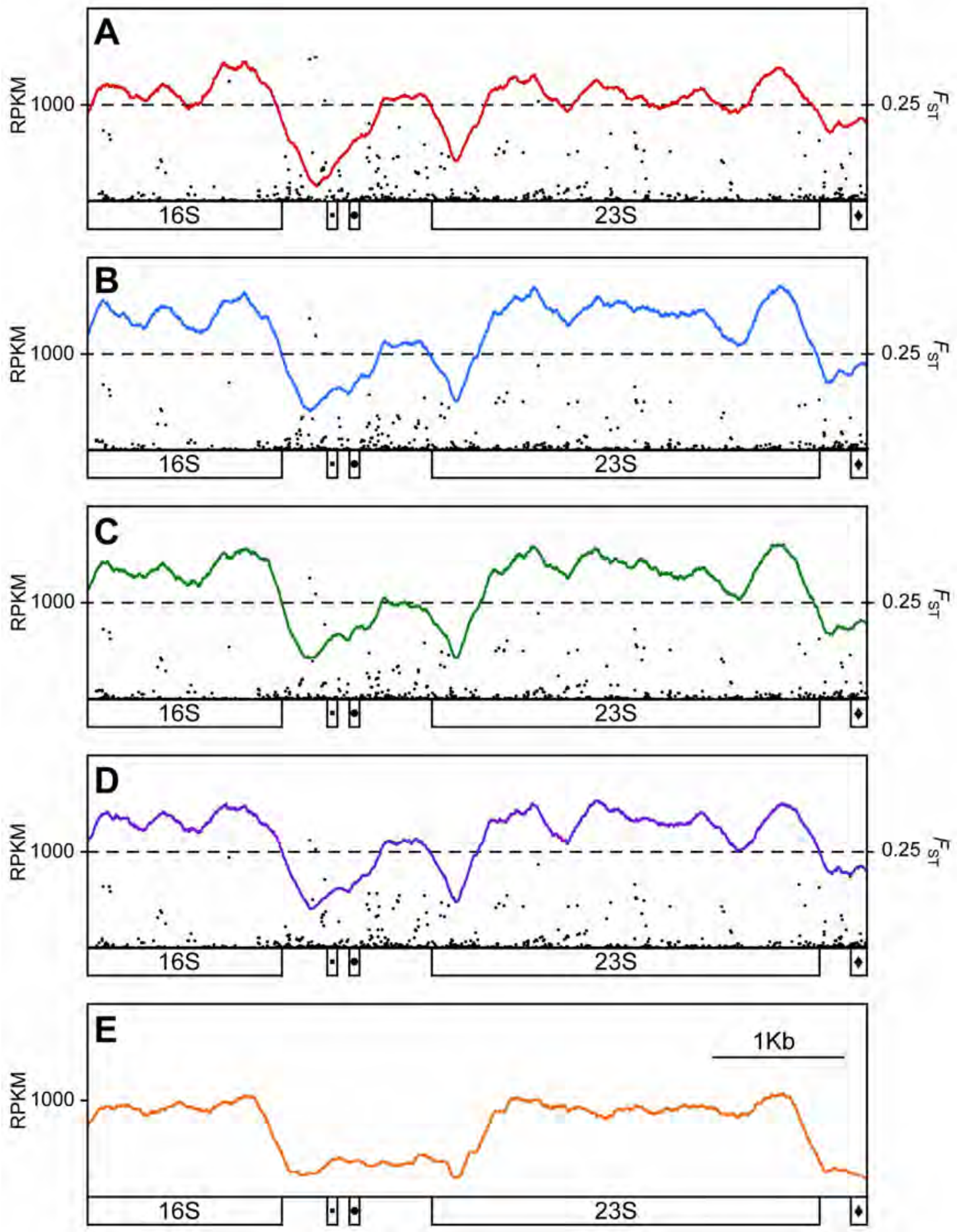

Figure 2.5. Conservation and diversity of the 16S-23S rRNA genomic region in the four plantselected rhizobial populations and soil population. A. Pea, B. Lentil, C. Fava bean, D. Vetch and E. Soil. Coverage (RPKM) along the reference genome sequence is indicated by a continuous colored line. SD-SNP location and $F_{\mathrm{ST}}$ values are indicated as dots. A discontinuous horizontal line indicates the $F_{\mathrm{ST}}>0.25$ threshold for SD-SNPs. The position of Ile-tRNA, Ala-tRNA and 5S rRNA in the reference genome sequence is indicated by boxes containing a small square, a large dot and a diamond, respectively. 
the reads recruited possessed a $\mathrm{C}$, which translated to a $F_{\mathrm{ST}}$ of 0.3122 . The other SDSNP was in the $23 S$ gene (position 802), with all the pea reads presenting an A, while $\mathrm{A} / \mathrm{C}$ was found the in soil population was $\left(F_{\mathrm{ST}}=0.2596\right)$.

Table 2.6. $F_{\mathrm{ST}}$ values for SD-SNPs in the rRNA region.*

\begin{tabular}{lcccc}
\hline SD-SNPs position & Pea & Lentil & Fava bean & Vetch \\
\hline 16S (1,069) & $\mathbf{0 . 3 1 2 2}$ & 0.1762 & 0.1386 & 0.2385 \\
16S-IletRNA (205) & $\mathbf{0 . 3 7 0 6}$ & $\mathbf{0 . 3 4 5 1}$ & $\mathbf{0 . 3 1 4 8}$ & $\mathbf{0 . 2 8 1 5}$ \\
16S-IletRNA (251) & $\mathbf{0 . 3 7 6 0}$ & $\mathbf{0 . 3 0 0 1}$ & $\mathbf{0 . 2 7 4 2}$ & $\mathbf{0 . 2 5 9 2}$ \\
16S-IletRNA (315) & $\mathbf{0 . 2 6 2 7}$ & $\mathbf{0 . 2 5 4 0}$ & 0.1967 & 0.1801 \\
23S (802) & $\mathbf{0 . 2 5 9 6}$ & 0.2219 & 0.2251 & 0.2182 \\
\hline
\end{tabular}

Figures in boldface are highly significant $\left(F_{S T}>0.25\right)$

Table 2.7. Nucleotide frequencies of SD-SNPs in the rRNA region.

\begin{tabular}{lcccccc}
\hline SD-SNPs position & SNP & Pea & Lentil & Fava bean & Vetch & Soil \\
\hline \multirow{2}{*}{ 16S (1,069) } & T & $\mathbf{0 . 8 2}$ & 0.67 & 0.62 & 0.73 & 0.21 \\
& C & 0.18 & 0.33 & 0.37 & 0.23 & $\mathbf{0 . 7 2}$ \\
\hline \multirow{2}{*}{ 16S-IletRNA (205) } & T & $\mathbf{0 . 9 4}$ & $\mathbf{0 . 9 2}$ & $\mathbf{0 . 9 0}$ & $\mathbf{0 . 8 7}$ & 0.36 \\
& G & 0.06 & 0.08 & 0.10 & 0.13 & $\mathbf{0 . 6 4}$ \\
\hline \multirow{2}{*}{ 16S-IletRNA (251) } & G & $\mathbf{0 . 9 3}$ & $\mathbf{0 . 8 7}$ & $\mathbf{0 . 8 5}$ & $\mathbf{0 . 8 3}$ & 0.33 \\
& A & 0.07 & 0.13 & 0.15 & 0.16 & $\mathbf{0 . 6 6}$ \\
\hline \multirow{2}{*}{ 16S-IletRNA (315) } & G & $\mathbf{0 . 9 4}$ & $\mathbf{0 . 9 3}$ & 0.88 & 0.87 & 0.47 \\
& A & 0.06 & 0.07 & 0.11 & 0.13 & $\mathbf{0 . 5 3}$ \\
\hline \multirow{2}{*}{ 23S (802) } & A & $\mathbf{1}$ & 0.98 & 0.98 & 0.97 & $\mathbf{0 . 5 9}$ \\
& C & 0 & 0.02 & 0.02 & 0.03 & $\mathbf{0 . 4 1}$ \\
\hline
\end{tabular}




\subsubsection{2. nod cluster}

A similar analysis of Pool-Seq populations was carried out with the nod region. Figure 2.6 shows the read coverage and $F_{S T}$ pairwise comparison against soil population along a stretch of 15,993 bp from symbiotic plasmid pRL10 that includes the nodOTNMLEFDABCIJ genes. Large differences in the coverage profile along nod cluster were found between soil and host. A considerable decrease was found in soil Pool-Seq along nod cluster, from 300 RPKM to 100 RPKM; doubtless, this reflects the symbiotic profile of the soil population, where just $46 \%$ of the population was symbiotic
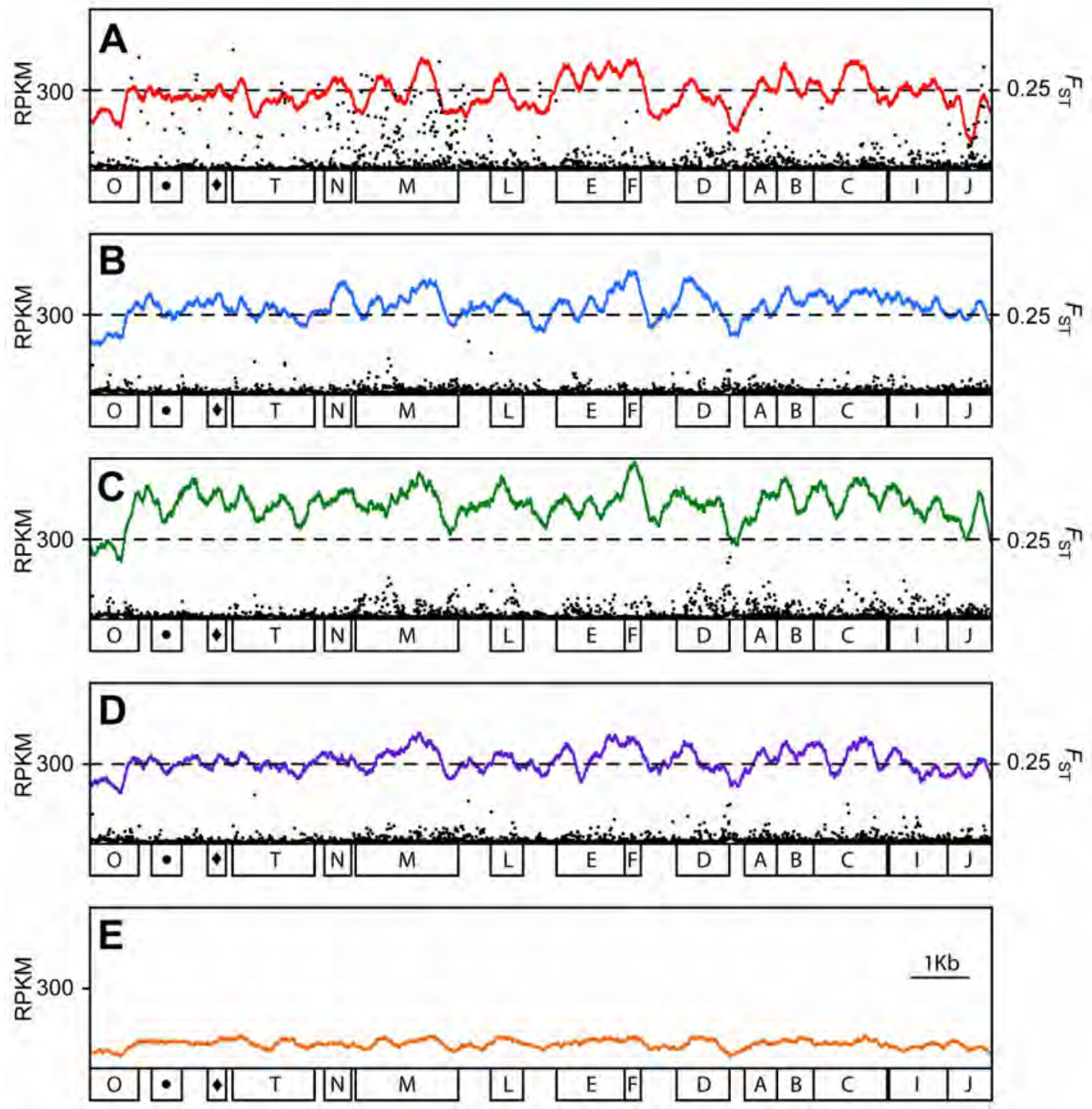

Figure 2.6. Conservation and diversity of the nod cluster symbiotic region in the four plantselected rhizobial populations and soil population. A. Pea, B. Lentil, C. Fava bean, D. Vetch and E. Soil. Coverage (RPKM) along the reference genome sequence is indicated by a continuous colored line. SD-SNP location and $F_{\mathrm{ST}}$ values are indicated as dots. A discontinuous horizontal line indicates the $F_{\mathrm{ST}}>0.25$ threshold, for SD-SNPs. The position of genes pRL100176 and pRL100177 is indicated as boxes containing a dot and a diamond, respectively. 
in Vicia sativa. Only 19 SD-SNPs were found between pea and soil populations, and the $F_{\mathrm{ST}}$ values for each significant position are shown in Table 2.8. Five of them were

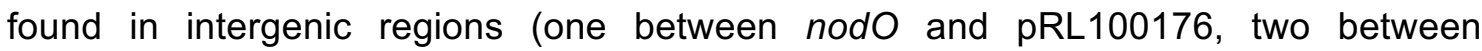
pRL100176 and pRL100177, one between $\operatorname{nod} T$ and nodN, two between nodM and nod $L$ and one between nodL and nodE), while the remaining fourteen were localized within coding regions (one in nodO, one in PRL100176, two in nodT, five in nodM, one

Table 2.8. $F_{\mathrm{ST}}$ values for SD-SNPs in the nod cluster.

\begin{tabular}{lcccc}
\hline SD-SNPs position & Pea & Lentil & Fava bean & Vetch \\
\hline nodO (727) & $\mathbf{0 . 2 8 8 2}$ & 0.0372 & 0.0079 & 0.0372 \\
nodO-pRL100176 (6) & $\mathbf{0 . 3 5 2 5}$ & 0.0562 & 0.0093 & 0.0589 \\
pRL100176 (129) & $\mathbf{0 . 2 5 6 0}$ & 0.0200 & 0.0046 & 0.0324 \\
pRL100176-7 (250) & $\mathbf{0 . 2 9 8 2}$ & 0.0192 & 0.0051 & 0.0412 \\
pRL100176-7 (253) & $\mathbf{0 . 2 8 2 4}$ & 0.0214 & 0.0064 & 0.0480 \\
nodT $(1,445)$ & $\mathbf{0 . 3 7 6 2}$ & 0.0116 & 0.0054 & 0.0572 \\
nodT $(592)$ & $\mathbf{0 . 2 7 3 8}$ & 0.0146 & 0.0194 & 0.0129 \\
nodT-nodN (53) & $\mathbf{0 . 2 6 7 9}$ & 0.0050 & 0.0050 & 0.0168 \\
nodM $(1,734)$ & $\mathbf{0 . 2 9 8 4}$ & 0.0067 & 0.0095 & 0.0184 \\
nodM $(1,644)$ & $\mathbf{0 . 2 9 6 7}$ & 0.0226 & 0.0110 & 0.0524 \\
nodM $(1,548)$ & $\mathbf{0 . 2 8 1 7}$ & 0.0086 & 0.0130 & 0.0339 \\
nodM $(875)$ & $\mathbf{0 . 2 5 7 5}$ & 0.0253 & 0.0053 & 0.0423 \\
nodM $(346)$ & $\mathbf{0 . 3 3 7 9}$ & 0.0298 & 0.0258 & 0.0366 \\
nodM-nodL (32) & $\mathbf{0 . 2 5 1 9}$ & 0.0181 & 0.0067 & 0.0548 \\
nodM-nodL (76) & $\mathbf{0 . 2 6 6 2}$ & 0.0244 & 0.0069 & 0.0492 \\
nodL-nodE (290) & $\mathbf{0 . 2 7 3 1}$ & 0.0115 & 0.0047 & 0.0350 \\
nodC $(1,173)$ & $\mathbf{0 . 2 6 0 1}$ & 0.0136 & 0.0062 & 0.0237 \\
nodJ (586) & $\mathbf{0 . 2 6 6 1}$ & 0.0059 & 0.0094 & 0.0181 \\
nodJ $(621)$ & $\mathbf{0 . 3 2 2 7}$ & 0.0208 & 0.0144 & 0.0584 \\
\hline
\end{tabular}

in nodC and two in nodJ). Table 2.9 shows SNP frequencies for each SD-SNP along the nod cluster. All these SD-SNP positions appeared to have two possible nucleotides with frequencies from 0.41 to 0.59 in the soil population, whereas in the pea subpopulation only one nucleotide was detected with frequencies from 0.93 to 1 ; this reflects the highly significant differences between soil and pea Pool-Seqs in these positions. However, no SD-SNP was found within the nod cluster between soil and the remaining host subpopulations (lentil, fava bean and vetch). It is noteworthy that the vetch subpopulation possessed the same nucleotides found in the pea subpopulation but with a lower range of frequencies from 0.6 to 0.7 , while in fava bean subpopulation in most of these positions, two possible nucleotides were found with frequencies from 0.56 to 0.44 , just as with the soil population, with the exception of SNPs localized in nodT position 592 and nodM position 346, where SNP frequencies ranged from 0.61 to 0.64. In lentil subpopulation an intermediate situation was found, with eleven SNPs having only one possible nucleotide (and frequencies of 0.6 to 0.68 ), while the 
remaining eight had frequencies from 0.41 to 0.59 (Table 2.9). Examination of SDSNPs between pea and soil Pool-Seqs localized in coding regions showed that six of them were non-synonymous, one in nodO, two in nodT, two in nodM and one in nodJ (Table 2.9).

Table 2.9. Nucleotide frequencies of SD-SNPs in the nod cluster. GLU (Glutamate), LYS (Lysine), ARG (Arginine), PRO (Proline), LEU (Leucine), PHE (Phenylalanine), GLN (Glutamine), ILE (Isoleucine), ASP (Asparagine), VAL (Valine) and ALA (Alanine).

\begin{tabular}{|c|c|c|c|c|c|c|}
\hline SD-SNPs position & SNP & Pea & Lentil & Fava bean & Vetch & Soil \\
\hline \multirow{2}{*}{ nodo (727) } & GAA $(\mathrm{GLU})^{*}$ & 0.98 & 0.66 & 0.56 & 0.69 & 0.51 \\
\hline & AAA (LYS) & 0.01 & 0.28 & 0.44 & 0.31 & 0.48 \\
\hline \multirow{2}{*}{ nodO-pRL100176 (6) } & $T^{*}$ & 0.96 & 0.64 & 0.48 & 0.65 & 0.41 \\
\hline & $\mathrm{A}$ & 0.04 & 0.36 & 0.52 & 0.35 & 0.59 \\
\hline \multirow{2}{*}{ pRL100176 (129) } & $\mathbf{G}^{*}$ & 0.97 & 0.66 & 0.55 & 0.70 & 0.54 \\
\hline & C & 0.03 & 0.34 & 0.45 & 0.30 & 0.46 \\
\hline \multirow{2}{*}{ pRL100176-7 (253) } & $\mathrm{C}^{*}$ & 0.98 & 0.63 & 0.54 & 0.70 & 0.51 \\
\hline & G & 0.02 & 0.37 & 0.46 & 0.30 & 0.49 \\
\hline \multirow{2}{*}{ pRL100176-7 (256) } & $A^{*}$ & 0.97 & 0.63 & 0.54 & 0.71 & 0.50 \\
\hline & G & 0.03 & 0.37 & 0.45 & 0.29 & 0.50 \\
\hline \multirow{2}{*}{$\operatorname{nod} T$ (592) } & CTT $($ LEU)* & 0.97 & 0.61 & 0.63 & 0.60 & 0.50 \\
\hline & TTT (PHE) & 0.03 & 0.36 & 0.37 & 0.40 & 0.50 \\
\hline \multirow{2}{*}{$\operatorname{nod} T(1,445)$} & CCA (PRO)* & 0.99 & 0.53 & 0.48 & 0.67 & 0.44 \\
\hline & CAA (GLN) & 0.01 & 0.47 & 0.52 & 0.33 & 0.56 \\
\hline \multirow{2}{*}{$\operatorname{nod} T-\operatorname{nod} N(53)$} & $\mathrm{A}^{*}$ & 0.98 & 0.55 & 0.53 & 0.64 & 0.53 \\
\hline & G & 0.02 & 0.45 & 0.47 & 0.36 & 0.47 \\
\hline \multirow{2}{*}{$\operatorname{nod} M(346)$} & ACC $(\mathrm{THR})^{*}$ & 1 & 0.66 & 0.64 & 0.68 & 0.50 \\
\hline & GCC (ALA) & 0 & 0.34 & 0.36 & 0.32 & 0.50 \\
\hline \multirow{2}{*}{ nodM (875) } & AGC (SER)* & 0.97 & 0.68 & 0.56 & 0.72 & 0.54 \\
\hline & ACC (THR) & 0.03 & 0.32 & 0.44 & 0.27 & 0.46 \\
\hline \multirow{2}{*}{ nodM $(1,548)$} & ATT (ILE)* & 0.95 & 0.51 & 0.54 & 0.64 & 0.47 \\
\hline & ATC (ILE) & 0.05 & 0.49 & 0.45 & 0.36 & 0.53 \\
\hline \multirow{2}{*}{ nodM $(1,644)$} & GAA (GLU)* & 0.94 & 0.57 & 0.50 & 0.65 & 0.43 \\
\hline & GAG (GLU) & 0.06 & 0.43 & 0.50 & 0.35 & 0.57 \\
\hline \multirow{2}{*}{ nodM $(1,734)$} & CTC (LEU)* & 1 & 0.59 & 0.61 & 0.66 & 0.54 \\
\hline & CTG (LEU) & 0 & 0.41 & 0.39 & 0.34 & 0.46 \\
\hline \multirow{2}{*}{ nodM-nodL (32) } & $\mathrm{A}^{*}$ & 0.94 & 0.60 & 0.51 & 0.71 & 0.49 \\
\hline & $\mathrm{C}$ & 0.06 & 0.39 & 0.49 & 0.29 & 0.51 \\
\hline \multirow{2}{*}{ nodM-nodL (76) } & $\mathrm{C}^{*}$ & 0.97 & 0.67 & 0.49 & 0.73 & 0.53 \\
\hline & G & 0.03 & 0.33 & 0.51 & 0.27 & 0.47 \\
\hline \multirow{2}{*}{ nodL-nodE (290) } & $A^{*}$ & 0.97 & 0.61 & 0.54 & 0.69 & 0.52 \\
\hline & C & 0.03 & 0.39 & 0.46 & 0.03 & 0.48 \\
\hline \multirow{2}{*}{$\operatorname{nod} C(1,173)$} & GAT (ASP)* & 0.93 & 0.57 & 0.52 & 0.61 & 0.47 \\
\hline & GAC (ASP) & 0.07 & 0.43 & 0.48 & 0.39 & 0.53 \\
\hline \multirow{2}{*}{ nodJ (586) } & GTA (VAL)* & 1 & 0.59 & 0.53 & 0.69 & 0.58 \\
\hline & ATA (ILE) & 0 & 0.41 & 0.47 & 0.31 & 0.42 \\
\hline \multirow{2}{*}{ nodJ (621) } & GCC (ALA)* & 0.96 & 0.57 & 0.54 & 0.68 & 0.56 \\
\hline & GCG (ALA) & 0.04 & 0.43 & 0.46 & 0.31 & 0.44 \\
\hline
\end{tabular}

* Nucleotide and amino acid present in the reference genome Rhizobium leguminosarum bv. viciae 3841. 


\subsubsection{Global $F_{\mathrm{ST}}$ analysis of Pool-Seq coding sequences}

A total of 7,266 Coding DNA sequence (CDSs) were analysed. Genes with coverage lower than 20 RPKM were considered to be absent and omitted from this analysis. The same tendency observed for total SD-SNPs was observed for CDSs, where the pea subpopulation presented 247 significantly different CDSs (SD-CDSs) with respect to soil, whereas lentil, fava bean and vetch had ten times less SD-CDSs (20, 32 and 30 , respectively). A Venn diagram was constructed in order to find SDCDSs shared by all subpopulations, as well as SD-CDSs exclusively host-selected for each subpopulation (Figure 2.7). Fifteen CDSs were significantly different for all subpopulations when compared to soil, and are listed in Table 2.10. Among them, CDSs with different functions were detected: enzymes, component of $A B C$ transporters, metX (homoserine O-acetyltransferase), a transposase and hypothetical proteins.

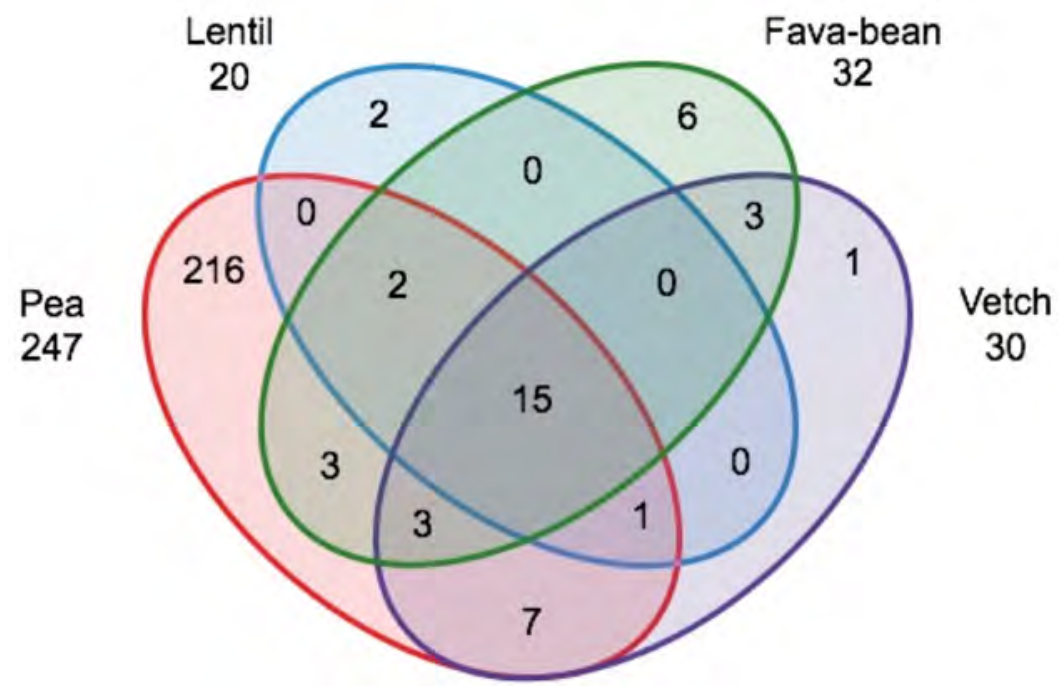

Figure 2.7. Shared and exclusive SD-CDSs for each host-selected subpopulations based on $F_{\mathrm{ST}}$ pairwise values re. soil population.

Each subpopulation exhibited different numbers of exclusive SD-CDSs: 216 for pea, 2 for lentil, 6 for fava bean, and 1 for vetch (Supplementary Table 2.S2). Among the 216 pea SD-CDSs there were genes with different functions: numerous components of ABC-transporters, the fixS copy from pRL9, EPS synthesis ( $p s s N)$, flgE (flagellar hook protein), a component of ATP synthase (atp/F), rpoE and pRL10 repB. Only two exclusive SD-CDSs were found in the lentil subpopulation, an oxidoreductase and a putative lipopolysaccharide biosynthesis O-acteyl transferase (wbbJ). Six SDCDSs were exclusive for the fava bean subpopulation, and were located in two 
clusters, a T6SS from pRL12 (impEDC) and tra from pRL7 (traDC). Only one SD-CDS was found for vetch, a hypothetical protein.

Table 2.10. Exclusive selected genes for all subpopulation

\begin{tabular}{ll}
\hline Product & Gene \\
\hline conserved hypothetical protein & $\mathrm{pRL} 100012$ \\
hypothetical protein & $\mathrm{pRL} 100107$ \\
putative acetolactate synthase, pseudogene & $\mathrm{pRL} 100115$ \\
putative permease component of ABC transporter, HAAT family & $\mathrm{pRL} 100130$ \\
putative substrate-binding component of ABC transporter, HAAT family & $\mathrm{pRL} 100131$ \\
putative homoserine O-acetyltransferase $($ met $X)$ & $\mathrm{pRL} 100137$ \\
putative non-heme chloroperoxidase $(c p o 2)$ & $\mathrm{pRL} 110136$ \\
putative cyclase/dehydrase & $\mathrm{pRL} 110137$ \\
conserved hypothetical protein & $\mathrm{pRL} 110464$ \\
conserved hypothetical protein & $\mathrm{pRL} 110465$ \\
putative transposase family protein & $\mathrm{pRL} 110607$ \\
putative transmembrane protein & $\mathrm{pRL} 120478$ \\
conserved hypothetical exported protein & $\mathrm{pRL} 120479$ \\
conserved hypothetical protein & $\mathrm{pRL} 70004$ \\
hypothetical protein & $\mathrm{RL} 2677$ \\
\hline
\end{tabular}

\subsubsection{0. rpoB sequences in the soil Pool-Seq}

In order to evaluate the Pool-Seq method in this experiment, the obtained SNP frequencies within the $r p o B$ gene were compared in both Pool-Seq data and in individual sequences obtained by the Sanger methodology (see 1.3). Only a subset of the soil isolates, closely related to the reference genome (groups $\mathrm{N}-\mathrm{Z}$ ) was used, because only reads above $85 \%$ identity aligned against the reference genome (Jorrín and Imperial, 2015b). Figure 2.8 shows polymorphic sites present in both data sets. A total of 33 SNPs were analysed. Twenty-two SNPs were found in the Pool-Seq sequence and 32 in the individual Sanger sequences; both samples shared 21 SNPs. Eleven of the SNPs appeared only in individual Sanger sequences, and always with frequencies lower than 0.1. One SNP was exclusive for the Pool-Seq analysis, with a frequency of 0.16 . Differences in frequencies between Pool-Seq and Sanger sequences were always below $17 \%$ for all the SNPs investigated. 


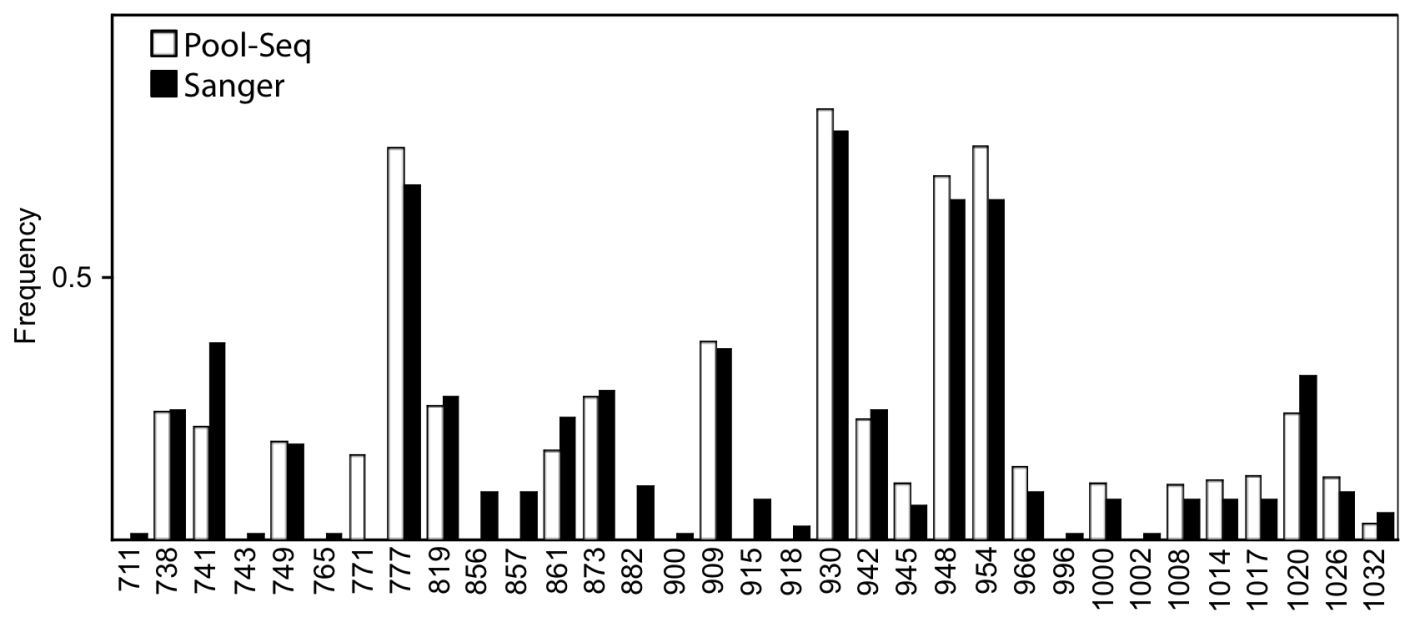

Figure 2.8. Distribution of polymorphic sites in the partial $r p o B$ sequence (447 nt) used for genotyping that are present in the Pool-Seq (white columns) and Sanger (black columns) soil datasets. $\mathrm{X}$ axis shows polymorphic $r p o B$ positions and $\mathrm{Y}$ axis represents SNP frequency.

\subsubsection{Pool-Seq analysis using other, closely-related genomes as reference}

Possible biases due to the specific reference genome used in Pool-Seq reading recruitment were assessed through the use of other, closely related genomes: Rhizobium leguminosarum bv. viciae WSM1455 (Reeve et al., 2015), $R$. leguminosarum bv. viciae WSM1481 (Reeve et al., 2015) and R. leguminosarum bv. viciae UPM791 (Sanchez-Cañizares et al, in preparation), Table 2.11 describes their genomic characteristics. These new reference genomes have sizes similar to that of $R$. leguminosarum bv. viciae 3841 , from 7.5 to $7.8 \mathrm{Mb}$. They also have comparable GC \% (ca. $61 \%$ ) and number of genes $(\approx 7,400)$. They differ in genome organization,

Table 2.11. Reference genome characteristics

\begin{tabular}{lcccc}
\hline & Rlv 3841 & Rlv UPM791 & Rlv WSM1455 & Rlv WSM1481 \\
\hline Genome size (Mb) & 7.755 & 7.834 & 7.557 & 7.555 \\
\hline Chromosome size (Mb) & 5.075 & 4.760 & 5.656 & 4.993 \\
No. plasmids & 6 & 5 & 2 & 5 \\
\hline & 0.870 & 1.291 & 1.657 & 1.025 \\
& 0.684 & 0.597 & 0.243 & 0.77 \\
Plasmids size (Mb) & 0.488 & 0.564 & & 0.332 \\
& 0.352 & 0.405 & & 0.246 \\
& 0.151 & 0.217 & & 0.240 \\
\hline GC \% & 0.147 & & & \\
No. genes & 60.86 & 61.00 & 60.93 & 60.98 \\
rRNA & 7,447 & 7,490 & 7,494 & 7,464 \\
tRNA & 9 & 9 & 6 & 9 \\
No. COGs & 58 & 57 & 48 & 55 \\
\hline
\end{tabular}


chromosome size (4.7-5.6 Mb) and number of plasmids (2-6). The extent of their similarity was calculated as ANIm values (Table 2.12). R. leguminosarum bv. viciae WSM1455, $R$. leguminosarum bv. viciae WSM1481 and $R$. leguminosarum bv. viciae 3841 appear to be in the same species, because their pairwise ANIm values are above 0.94. On the contrary, R. leguminosarum bv. viciae UPM791 appears to belong to a different genomic species because it presented ANIm values lower than 0.94 for all pairwise comparisons (Richter and Rosselló-Móra, 2009).

Table 2.12. ANIm values among reference genomes

\begin{tabular}{lllll}
\hline & Rlv 3841 & Rlv UPM791 & Rlv WSM1455 & Rlv WSM1481 \\
\hline Rlv 3841 & 1 & 0.9304 & 0.9596 & 0.9594 \\
Rlv UPM791 & 0.9305 & 1 & 0.9292 & 0.9292 \\
Rlv WSM1455 & 0.9596 & 0.9292 & 1 & 0.9858 \\
Rlv WSM1481 & 0.9594 & 0.9292 & 0.9858 & 1 \\
\hline
\end{tabular}

Reads were recruited for each Pool-Seq dataset against each $R$. leguminosarum bv. viciae reference genome, and the fraction of aligned reads was calculated (Table 2.13). For all Pool-Seqs, alternative reference genomes recruited lower numbers of reads than those recruited by $R$. leguminosarum bv. viciae 3841, suggesting that each of the Pool-Seqs is more similar to R. leguminosarum bv. viciae 3841 than to any other reference genome. Despite the reduction in recruitment, the same patterns of variability were observed, with pea subpopulations exhibiting the highest recruitment, followed by vetch, lentil and fava bean, and, finally, soil population.

Table 2.13. Percentage of aligned reads in each $R$. leguminosarum bv. viciae reference genome for each Pool-Seq.

\begin{tabular}{lcccc}
\hline & Rlv 3841 & Rlv UPM791 & Rlv WSM1455 & Rlv WSM1481 \\
\hline Pea & 84.17 & 59.13 & 75.37 & 75.41 \\
Lentil & 77.78 & 57.08 & 70.91 & 71.02 \\
Fava bean & 77.23 & 56.98 & 71.25 & 71.73 \\
Vetch & 81.87 & 58.30 & 74.4 & 74.42 \\
Soil & 45.99 & 35.95 & 43.4 & 43.45 \\
\hline
\end{tabular}


Genome-wide abundances of SD-SNPs were estimated for each Pool-Seq dataset against each of the $R$. leguminosarum bv. viciae reference genomes (Table 2.14). Although $R$. leguminosarum bv. viciae 3841 showed the highest levels of recruitment, it also showed the highest numbers of SD-SNPs. Irrespective of the reference genome used, the pea subpopulation presented a higher proportion of SD-SNPs in pairwise comparisons with the soil population. With R. leguminosarum bv. viciae UPM791 as reference, the pea SD-SNPs were three-times more abundant, while they were tentimes more abundant with $R$. leguminosarum bv. viciae $3841, R$. leguminosarum bv. viciae WSM1455 and $R$. leguminosarum bv. viciae WSM1481.

Table 2.14. Genome-wide abundance of significantly different single nucleotide polymorphisms in Pool-Seq DNA samples respect to soil Pool-Seq expressed in percentage.

\begin{tabular}{lllll}
\hline & Rlv 3841 & Rlv UPM791 & Rlv WSM1455 & Rlv WSM1481 \\
\hline Pea & 1.256 & 0.290 & 0.297 & 0.298 \\
Lentil & 0.078 & 0.041 & 0.036 & 0.037 \\
Fava bean & 0.063 & 0.025 & 0.015 & 0.014 \\
Vetch & 0.134 & 0.101 & 0.070 & 0.070 \\
\hline
\end{tabular}




\subsection{DISCUSSION}

\subsubsection{The Rhizobial soil population}

Total counts of bacteria on MNBP medium were, in our hands, one order of magnitude lower than those obtained by Louvrier and collaborators in 1995. Our P1 soil was collected in Dijon in 2010 , transported to Madrid and stored at room temperature in a non-air-conditioned storage room (average relative humidity for Madrid of $57 \%$, www.aemet.es). Gram-negative bacteria are notoriously less droughttolerant than gram-positive bacteria and fungi because they have thinner, less rigid cell walls and comparably lower levels of compatible solutes that enhance osmoregulatory capabilities (Schimel et al., 1989, Kempf and Bremer, 1998, Fierer et al., 2003). This can explain that the percentage of microorganisms that were unable to grow on LB medium was higher in our case (43\% versus $10 \%$ in (Louvrier et al., 1995). Despite these differences, the total counts of Rhizobium spp. appear to have been maintained during 25 years $\left(10^{4} \mathrm{cfu} \cdot \mathrm{g}^{-1}\right)$; these bacteria have been described as very resistant to dessication in view of their ability to synthetize: antioxidants, such as catalases; osmolites; stress-resistance proteins; and exopolysaccharides (Goyal et al., 1986, Vanderlinde et al., 2010). Slightly over one-half (56.8\%) of the rhizobia isolated by us were able to establish effective symbioses. Different ratios of symbiotic vs. nonsymbiotic rhizobia have been reported in different soils, from 100\% (Bromfield et al., 1995) to $\approx 2 \%$ (Segovia et al., 1991) of symbiotic isolates. These differences could be due to different rhizobial soil compositions, but also to the screening methods used. Previous studies with the $\mathrm{P} 1$ soil showed that out of a total of thirteen $R$. leguminosarum strains isolated directly from soil, five (ca. 40\%) were symbiotic and able to form nodules in V. sativa, T. repens or $P$. vulgaris (Laguerre et al., 1993a). Our results showed that $66(55.93 \%)$ of a total of 118 isolates were able to nodulate at least one of the tested plants ( $V$. sativa, $T$. repens or $P$. vulgaris). This compares with $2.04 \cdot 10^{4} \mathrm{cfu} \cdot \mathrm{g}^{-1}(54.23 \%)$ from the total number $\left(3.76 \cdot 10^{4} \mathrm{cfu} \cdot \mathrm{g}^{-1}\right) \mathrm{fnrN}$-positive bacteria that were positive for at least one of the symbiotic markers. Therefore, the differences in the proportion in symbiotic/non-symbiotic bacteria would be due to the total amount of bacteria isolated (13 versus 118 ) that could enhance the small differences. None of the bacterial isolates defined as non-symbiotic by the semi-selective and genotyping screening methodology were able to nodulate any of the plants tested, which highlights and validates the accurate design of symbiotic screening markers.

Key to our screening procedure for Rhizobium was the successful PCR amplification of the fnrN gene; a BLASTP search with FnrN within the Genbank database showed no strains outside the Rhizobiaceae family with higher than $64 \%$ 
identity with the chromosomal fnrN gene of $R$. leguminosarum bv. viciae 3841 gene (RL2818). However, the second rhizobial marker, glnIl (RL3549) appeared to be more widespread: BLASTP searches with GInll showed that members of the Mesorhizobium genus contain glnll genes with an identity of $93 \%$. This high conservation of $\mathrm{g} / \mathrm{n} / \mathrm{l}$ among families of the Rhizobiales was reflected in the numbers of $\mathrm{glnll+}$ isolates (Table 2.2), which were ca. one order of magnitude higher $\left(1.16 \cdot 10^{5} \mathrm{cfu} \cdot \mathrm{g}^{-1}\right)$ than those of total Rhizobium sp. strains $\left(3.76 \cdot 10^{4} \mathrm{cfu} \cdot \mathrm{g}^{-1}\right)$, as defined by the $f n r N+$ character. Most of the strains obtained cluster within the Rhizobiaceae family (Figure 2.1, groups $E-Z$ ), with the exception of four isolates (groups $A-D$ ) that belong to the genus Phyllobacterium. No Phyllobacterium fnrN nucleotide sequences have been deposited in Genbank, but the genomes of three strains have been sequenced and are available in the JGI-IMG database (Phyllobacterium sp. YR531 (Brown et al., 2012), Phyllobacterium sp. CL33Tsu and Phyllobacterium sp. UNC302MFCol5.2). A BLASTP search within these genomes was performed using $\mathrm{FnrN}$ protein coding sequence and no hit with identity greater than $30 \%$ was obtained, which means that fnrN is absent from these genomes. Our laboratory has sequenced more than 100 rhizobial genomes (Imperial et al. unpublished data), including these four Phyllobacterium-related soil strains (S03A21, S03A24, S03B44 and S07109). None of them showed any open reading frame similar to the fnrN gene; however, some sequences showed similarity to one of fnrN primers (BLASTN searches using each genome as database). Thus, genomic information does not explain why these strains were PCR-positive for fnrN in the screening. It is possible that sequences unrelated $f n r N$ were amplified, although the available information drawn from the genome draft assemblies did not shed any light on this artefact. It is noteworthy that a high number of the isolated strains were phylogeneticaly associated to $R$. alamii (Figure 2.1 , groups I and J), with $49 \%$ of all the non-symbiotic isolates belonging to this group (25 out of 51). As already mentioned in the introduction to this chapter, $R$. alamii has been isolated from the rhizospheres of legumes and non-legumes plants, and appears to be a common root-associated rhizobium (Alami et al., 2000, Berge et al., 2009). Additionally, a high number of groups clustered with Rhizobium leguminosarum-related strains (groups N-Z, Figures 2.1 and 2.2). These isolates showed a high $r p o B$ diversity (Figure 2.2), and not all $R$. leguminosarum strains clustered together: at least three differentiated clusters could be defined, those of group $\mathrm{N}$, group $\mathrm{O}$, and groups $\mathrm{P}-\mathrm{Z}$. Altogether, we were able to define 26 different $r p o B$ genotypes (A-Z), a large number, although smaller than the number of groups (33) defined previously by REP-PCR (Repetitive Extragenomic Palindromic PCR) in the same P1 soil (Laguerre et al., 2003), with the additional advantage that Sanger sequencing of $r p o B$ fragment was not only more informative, but more 
reproducible than REP-PCR (Laguerre et al., 2003). These results evidence $r p o B$ as a convenient marker to describe and discriminate closely-related Rhizobium strains allowing as well to elucidate their phylogenetic relationships. We have tested its potential as a marker by comparing $r p o B$ - and $16 \mathrm{~S}$ rRNA-derived phylogenetic trees (Supplementary Figures 2.S1). Remarkably, rpoB, besides reproducing the phylogenetic relationships among rhizobial strains, also improved the ability to differentiate among strains. This was due to the higher sequence variability $(62.5 \% \mathrm{vs}$. $18.3 \%$ polymorphic sites for $r p o B$ and $16 S$ rRNA, respectively), even when using a shorter fragment (504 nt for rpoB vs. 1,287 nt for $16 \mathrm{~S}$ rRNA). In conclusion, although the $r p o B$ potential as phylogenetic marker was already proposed before (Morse et al., 1996, Korczak et al., 2004, Adékambi et al., 2008), our work confirms this and also suggests it as an advantageous substitute for fragment polymorphism, PCR-based methods.

The availability of a number of $r p o B$ sequences from a number of rhizobial P1 soil isolates prompted us to examine the Pool-Seq data for $r p o B$ sequences. Pool-Seq analysis requires recruitment of reads to a reference genome, $R$. leguminosarum bv. viciae 3841 in our case, and we have previously shown that, under our standard analysis conditions, a minimum of ca. $85 \%$ identity with the reference genome is required for read recruitment (Jorrín and Imperial, 2015b). Therefore, it is not surprising that only those $r p o B$ soil sequences from strains related to $R$. leguminosarum bv. viciae 3841 (rpoB groups N-Z, Figures 2.1 and 2.2), showed similarity to the Pool-Seq alignment (Figure 2.8). This read identity limitation was also clearly reflected in the lower global coverage for the gene in the soil Pool-Seq: while host subpopulations showed $77.2-84.2 \%$ aligned reads (Chapter 1 ), in soil Pool-Seq only $49.96 \%$ reads were recruited. This drop in coverage also affected the differential presence of certain genes between soil and host subpopulations (Figure 2.5). Despite this limitation, some gene coverages were strikingly different between host and soil Pool-Seqs. This is the case of the $h s d M S R$ cluster $^{2}$ (type I restriction enzyme involved in host restriction / modification (Bertani and Weigle, 1953)), practically absent in the soil population and with coverages of 30-40 RPKM in the combined host Pool-Seq. Why a restriction / modification operon should be enriched in host-selected populations is puzzling and several experimental approaches to the problem come to mind. Previously, however, it would be interesting to study these gene sequences within individual genome sequences from non-symbiotic and symbiotic isolates, both from soil and from host nodules.

\footnotetext{
${ }^{2}$ There is another hsdMSR cluster in the chromosome (RL0097-RL0100) with RPKM $<20$ (absent) in all Pool-Seqs: pea, lentil fava bean, vetch and soil.
} 


\subsubsection{Host selection of genotypes from those available in the soil}

In Chapter 1 we were able to demonstrate that legume hosts prefer specific rhizobial genotypes from those available in the soil, and that this selection is dependent on the legume species. In this Chapter we have undertaken to determine the extent and limits of the variability of genotypes in soil in order to adequately frame those results. It is noteworthy that for fifteen CDSs, all host-selected subpopulations presented significantly different genotypes as compared with the soil population (Table 2.10). These fifteen CDSs that were shared by all host-selected subpopulations were significantly different from those genotypes presented in the soil populations. This selection was dependent on symbiosis establishment, but independent of the host plant.

However, host preference for specific genotypes was especially relevant in the case of pea plants that selected a Rhizobium leguminosarum population significantly different from that present in the soil. This choice of specific genotypes was documented through a large number of individual results (Figures 2.5- 2.7, Tables 2.52.14). Quite on the contrary, lentil and fava bean plants did not show a significant genotype selection after one life cycle, and their nodule rhizobial populations reflected that present in soil. Vetch plants revealed a certain genotypic preference, but not as substantial or as important as that from pea plants. Laguerre et al. (2003), in their ground-breaking study of plant selection of rhizobial genotypes showed that pea plants did not necessarily select the most abundant genotype in the soil; rather, they were able to discriminate among the diversity of soil rhizobial genotypes. This phenomenon had been already described in an earlier clover study by Zeze et al. (2001), and in a pea study by Bromfield et al. (1995) as well as for other rhizobia-legume systems (Hartmann et al., 1998). As mentioned above, lentil and fava bean reflect in their nodules a rhizobial population that was not significantly different from soil populations. This could have two possible explanations: 1) both legumes are non-selective for rhizobia, and all the diversity present in the soil is also present in the nodules, and 2) these plants selected the most abundant $R$. leguminosarum genotypes present in the soil. To discern between these two possible hypothesis it was found necessary to carry out experiments with multiple rounds of choice / selection (as in Chapter 3 mesocosm experiments). No previously published evidence reported fava bean and lentil as nonselective plants and, in fact, Laguerre et al. (2003) defined a fava bean nodule population that was significantly different from the soil population. If in fact, fava bean was a non-selective plant, this discrepancy could be explained in terms of the vast differences in resolution of the two population comparison methods used. In Laguerre 
et al. (2003) two PCR-RFLP markers were used to confirm a preliminary REP-PCR classification (based on a maximum of 25 bands in a gel): the intergenic spacer (IGS) region between $16 \mathrm{~S}$ and $23 \mathrm{~S}$ rRNA (up to eight different bands in a gel), and the nodD$F$ region (up to nine different bands in a gel), while we have used all the sequences that align to the reference genome $R$. leguminosarum bv. viciae 3841 , a method that was several orders of magnitude more sensitive.

The pea-selected rhizobial subpopulation accumulated a higher number of SDSNPs along all the tested reference genomes (Table 2.5 and 2.14), as well as the highest number of SD-CDSs compared to soil (Figure 2.7 and Supplementary Table 2.S2). The number of total SD-SNPs between pea and soil Pool-Seqs was the largest found (97,949 SD-SNPs), one order of magnitude higher than for any other host subpopulation (Table 2.5). In addition, the pea Pool-Seq also showed the highest number of SD-SNPs within the 16S rRNA-23S rRNA region, and in the nod cluster region, presumably a region submitted to high selective pressure due to its role in plant infection, SD-SNPs were present only in the pea subpopulation (Figure 2.5 and 2.6). These SD-SNPs in the symbiotic region were analysed in detail. It is noteworthy that, for every SD-SNP position within the nod cluster, where the soil population presented two different nucleotides (Table 2.9), the pea subpopulation clearly selected one of them, and this was always the same that is present in the reference genome $R$. leguminosarum bv. viciae 3841. Some SD-SNPs produced non-synonymous codon substitutions with the potential to alter the secondary structure of the protein, among them nodO (position 727) and nodT (position 1,445). NodO is a dimeric $\mathrm{Ca}^{2+}$-binding protein similar to the HlyA haemolysin from Eschericia coli. It forms cation-selective channels in artificial bilayers and is secreted in response to flavonoids, playing a role in symbiosis (Economou et al., 1990, Economou et al., 1994, Sutton et al., 1994). We have only been able to find the nodO gene in nod clusters from Rhizobium leguminosarum bv. viciae strains, where different NodOs share at least $78 \%$ of protein identity (BLASTP searches within the JGI/IMG genome database). No clear phenotype could be ascribed to NodO- mutants; however, in the absence of nodEF-required for host-specific C18:4 acyl group addition to Nod factor- nodO is essential for nodulation in pea and vetch (Downie and Surin, 1990). Moreover, in the absence of an active nodE, the nodO gene from $R$. leguminosarum bv. viciae enables Rhizobium leguminosarum bv. trifolii to nodulate vetch (Economou et al., 1994). $\mathrm{Ca}^{2+}$-binding proteins are known to have two conserved domains. The first one is RTX (Repeat in ToXin), an N-terminal, multiply-repeated nonapeptide rich in aspartic acid and glycine, present in many secreted toxin proteins, that form a ß-roll and coordinate binding of $\mathrm{Ca}^{2+}$ ions. (Ludwig et al., 1988, Economou et al., 1990, Baumann et al., 1993). The 
second conserved region is the C-terminal secretion domain, characterized by glutamic/aspartic acid residues in conserved positions ( $E / D X_{21-23} E / D X_{13} D$ ). This domain is essential for secretion of nodO trough the Type I secretion system prsDE (RL3656-8) (Sutton et al., 1996, Walker and Downie, 2000). We have found in nucleotide position 727 of the nodO gene a SD-SNP change from $G$ to $A$ that would change a glutamic acid (negatively charged) in amino acid position 243 (out of 284) to a lysine (positively charged) (Table 2.9). This change affects the first conserved glutamic acid of the secretion domain ( $\left.E / D X_{21-23} E / D X_{13} D\right)$. Most pea subpopulation Pool-Seq reads that aligned to nodO (1,013 reads) encoded a $G$ at position 727 , ie. a glutamic acid, in this site (0.98 SNP frequency), selecting it between the two possible genotypes presented in the soil population (G, 0.51 SNP frequency; and A, 0.48 SNP frequency). This result can be interpreted in terms of changes in NodO secretion that affect infectivity or effectivity in the pea host. On the contrary, the fava bean nodule subpopulation reflected the nodO soil composition, which could be interpreted as meaning that whatever the differences in secretion of both variants of NodO, they do not have an effect on the fava bean symbiosis. Vetch and lentil hosts showed only a slight preference for $\mathrm{G}$ at position 727 (E243; 0.66 and 0.69 frequencies, respectively). It has been reported that a partial deletion of the NodO C-terminal region (26 aas, 259284) decreased secretion by 98\% (Sutton et al., 1996); however swapping of the Cterminal regions of different hemolysin proteins, which display very little sequence conservation among them, produced functional proteins, suggesting that, at least for those proteins, there is little sequence specificity (Hui et al., 2000, Hui and Ling, 2002). Comparison of the C-terminal sequences of RTX proteins exported by type I secretion systems suggests that it favours non-positively charged residues, although it nevertheless appeared to be highly flexible (Delepelaire, 2004). The change of Glu to Lys at the C-terminal end could possibly affect the level of secretion, or maybe this change could be linked to other specific, host-selected genotypes. Given that NodO is secreted through the type I PrsDE secretion system (see above), we have analysed this region as well for SNPs: while no significant gene average $F_{\mathrm{ST}}$ was found in these genes, relatively high $F_{\mathrm{ST}}$ values compared to soil were found in the pea subpopulation (RL3656: 0.2, prsD: 0.21, prsE: 0.18). This is in contrast to the much lower values observed in the remaining plant-selected subpopulations (RL3656: 0.05, 0.04, 0.08; prsD: $0.07,0.05,0.09$; prsE: $0.07,0.05,0.1$; for lentil, fava bean and vetch, respectively).

NodT has been suggested to encode an outer membrane lipoprotein, able to be translocated through the inner membrane to the outer membrane, and which resembles TolC. NodT proteins possess two conserved domains: an $\mathrm{N}$-terminal signal 
peptide ending in a typical outer-membrane lipoprotein cleavage site, and a $C$ terminus conserved in most TolC-like proteins (Surin et al., 1990, Rivilla et al., 1995). However, mutations in nodT did not significantly affect nodulation in vetch (Surin et al., 1990), probably because another copy of these genes was found outside the symbiotic plasmid. This would explain the lack of nodulation-deficient phenotype (Rivilla and Downie, 1994). The nodT gene is not present in all nod clusters of Rhizobium leguminosarum bv. viciae strains, maybe due to its chromosomically-encoded redundancy (BLASTP, JGI/IMG genome database). The second copy is localized in the chromosome (gene RL3876), and shares $67 \%$ aas with the symbiotic copy. In the soil population, two possible SNPs were localized at position 1,445 within nodT, C (0.44 SNP frequency) and A (0.56 SNP frequency), affecting the last amino acid of the NodT protein. These two possible nucleotides translate into two different amino acids: proline and glutamine, respectively (Table 2.9). A clear selection for proline was observed in the pea subpopulation (0.98 SNP frequency), as well for vetch, but in this case differences are no significant (0.67 SNP frequency). Finally, the distribution of alternative nucleotides in nodT genes from lentil and fava bean subpopulations was similar to that in soil. A change from proline to glutamine or vice versa should be relevant; however, it lies outside the C-terminal conserved domain, and some of the reference genomes, such as $R$. leguminosarum bv. viciae WSM1481 and $R$. leguminosarum bv. viciae WSM1455, have a glutamine in this position.

This last observation prompted us to consider possible bias arising from the use of a specific reference genome, in our case $R$. leguminosarum bv. viciae 3841 . This was studied through the use of alternative, closely-related reference genomes, namely those of strains WSM1481, WSM1455 and UPM791 above. These strains are highly related to strain 3841. However, ANIm values between 3841 and any of the other two were lower than 0.94 , suggesting that they belong to different genospecies. This notwithstanding, the same tendencies were observed with any of the alternative reference genomes. Irrespective of the reference genome used, the pea subpopulation accumulated ten times more SD-SNPs than the remaining subpopulations compared to soil (Table 2.14). These analyses stress the different behaviour of plants that can be nodulated by $R$. leguminosarum bv. viciae with regard to the microsymbiont, where pea acted as a selective plant, while fava bean did not (after one cycle of selection), because it reproduced in its nodules the diversity described in soil. 
At this point it is important to note that our results on preference / selection of rhizobial genotypes were obtained after a single round of choice / selection by the plant, and that, perhaps, if this process were to be maintained along multiple cycles of plant infection and reproduction (seasons), different or exacerbated results would be obtained. This was the idea behind the experiments reported in Chapter 3 . 


\subsection{SUPPLEMENTARY MATERIAL}

Supplementary Table 2. S1. Screening genotype and nodulation phenotype of soilselected population. (1/3)

\begin{tabular}{|c|c|c|c|}
\hline Group & strain & Screening & Plant \\
\hline A & S03A24 & non-symbiotic & \\
\hline \multirow{2}{*}{ B } & S07109 & non-symbiotic & \\
\hline & S05054 & other & V.sativa \\
\hline C & S03B44 & non-symbiotic & \\
\hline $\mathrm{D}$ & S03A21 & non-symbiotic & \\
\hline \multirow{2}{*}{$\mathrm{E}$} & S03B49 & symbiotic & V.sativa \\
\hline & S03B27 & symbiotic & V.sativa \\
\hline \multirow{2}{*}{$\mathrm{F}$} & S07001 & other & T. repens and $P$. vulgaris \\
\hline & S11101 & other & T. repens \\
\hline G & S07051 & non-symbiotic & \\
\hline \multirow{2}{*}{$\mathrm{H}$} & S03A33 & non-symbiotic & \\
\hline & S04010 & non-symbiotic & \\
\hline \multirow{16}{*}{ I } & S01A03 & non-symbiotic & \\
\hline & S03B46 & non-symbiotic & \\
\hline & S04023 & non-symbiotic & \\
\hline & S04085 & non-symbiotic & \\
\hline & S05055 & non-symbiotic & \\
\hline & S05071 & non-symbiotic & \\
\hline & S06040 & non-symbiotic & \\
\hline & S08044 & non-symbiotic & \\
\hline & S09037 & non-symbiotic & \\
\hline & S10001 & non-symbiotic & \\
\hline & S10038 & non-symbiotic & \\
\hline & S10103 & non-symbiotic & \\
\hline & S11025 & non-symbiotic & \\
\hline & S11195 & non-symbiotic & \\
\hline & S12097 & non-symbiotic & \\
\hline & S08069 & non-symbiotic & \\
\hline \multirow{9}{*}{$\mathrm{J}$} & S04043 & non-symbiotic & \\
\hline & S06027 & non-symbiotic & \\
\hline & S06154 & non-symbiotic & \\
\hline & S09039 & non-symbiotic & \\
\hline & S09067 & non-symbiotic & \\
\hline & S10024 & non-symbiotic & \\
\hline & S10165 & non-symbiotic & \\
\hline & S13041 & non-symbiotic & \\
\hline & S13064 & non-symbiotic & \\
\hline $\mathrm{K}$ & S10058 & non-symbiotic & \\
\hline \multirow{3}{*}{$\mathrm{L}$} & S09123 & non-symbiotic & \\
\hline & S11017 & non-symbiotic & \\
\hline & S13031 & non-symbiotic & \\
\hline $\bar{M}$ & S06190 & non-symbiotic & \\
\hline
\end{tabular}


Supplementary Table 2. S1. Screening genotype and nodulation phenotype of soilselected population. $(2 / 3)$

\begin{tabular}{|c|c|c|c|}
\hline Group & strain & Screening & \\
\hline \multirow{5}{*}{$\mathrm{N}$} & S05061 & symbiotic & V.sativa \\
\hline & S10156 & symbiotic & V.sativa \\
\hline & S11115 & symbiotic & T. repens \\
\hline & S11190 & other & T. repens \\
\hline & S11200 & symbiotic & T. repens \\
\hline $\mathrm{O}$ & S09020 & symbiotic & T. repens \\
\hline $\mathrm{P}$ & S07007 & symbiotic & V.sativa \\
\hline Q & S12024 & symbiotic & V.sativa \\
\hline \multirow{15}{*}{$\mathrm{R}$} & S03B04 & symbiotic & V.sativa \\
\hline & S03B08 & symbiotic & V.sativa \\
\hline & S03B20 & symbiotic & V.sativa \\
\hline & S03B32 & symbiotic & V.sativa \\
\hline & S03B47 & other & V.sativa \\
\hline & S03B53 & other & V.sativa \\
\hline & S03C09 & symbiotic & V.sativa \\
\hline & S06034 & symbiotic & V.sativa \\
\hline & S06065 & symbiotic & V.sativa \\
\hline & S06136 & symbiotic & V.sativa \\
\hline & S06200 & symbiotic & V.sativa \\
\hline & S08022 & symbiotic & V.sativa \\
\hline & S10040 & symbiotic & V.sativa \\
\hline & S10054 & other & V.sativa \\
\hline & S13015 & symbiotic & V.sativa \\
\hline \multirow{4}{*}{ S } & S03B17 & symbiotic & V.sativa \\
\hline & S03B25 & symbiotic & V.sativa \\
\hline & S13042 & symbiotic & V.sativa \\
\hline & S10036 & symbiotic & V.sativa \\
\hline \multirow{5}{*}{$\mathrm{T}$} & S03A13 & symbiotic & V.sativa \\
\hline & S03B19 & symbiotic & V.sativa \\
\hline & S08013 & other & V.sativa \\
\hline & S11185 & symbiotic & V.sativa \\
\hline & S11031 & other & V.sativa \\
\hline \multirow{4}{*}{$U$} & S03A12 & non-symbotic & \\
\hline & S03A20 & symbiotic & V.sativa \\
\hline & S06190 & symbiotic & V.sativa \\
\hline & S03D22 & symbiotic & V.sativa \\
\hline \multirow{7}{*}{ V } & S05024 & non-symbotic & \\
\hline & S06057 & non-symbotic & \\
\hline & S10157 & non-symbotic & \\
\hline & S10158 & non-symbotic & \\
\hline & S11009 & non-symbotic & \\
\hline & S11064 & non-symbotic & \\
\hline & S12067 & non-symbotic & \\
\hline
\end{tabular}


Supplementary Table 2. S1. Screening genotype and nodulation phenotype of soilselected population. (3/3)

\begin{tabular}{|c|c|c|c|}
\hline Group & strain & Screening & \\
\hline \multirow{7}{*}{ W } & S01A39 & non-symbotic & \\
\hline & S03A23 & other & V.sativa \\
\hline & S09012 & symbiotic & V.sativa \\
\hline & S10022 & non-symbotic & \\
\hline & S11070 & non-symbotic & \\
\hline & S11184 & non-symbotic & \\
\hline & S13064 & non-symbotic & \\
\hline \multirow{2}{*}{$\mathrm{X}$} & S04022 & non-symbotic & \\
\hline & S07044 & non-symbotic & \\
\hline \multirow{9}{*}{$\mathrm{Y}$} & S05036 & symbiotic & V.sativa \\
\hline & S05037 & symbiotic & V.sativa \\
\hline & S05056 & symbiotic & V.sativa \\
\hline & S09003 & symbiotic & V.sativa \\
\hline & S09013 & symbiotic & V.sativa \\
\hline & S09014 & symbiotic & V.sativa \\
\hline & S09130 & symbiotic & V.sativa \\
\hline & S12076 & symbiotic & V.sativa \\
\hline & S12092 & symbiotic & V.sativa \\
\hline \multirow{15}{*}{ Z } & S03A30 & symbiotic & V.sativa \\
\hline & S03B01 & other & V.sativa \\
\hline & S03B10 & symbiotic & V.sativa \\
\hline & S03B21 & other & V.sativa \\
\hline & S03D26 & other & V.sativa \\
\hline & S04054 & other & V. sativa, T. repens and P. vulgaris \\
\hline & S05050 & symbiotic & V.sativa \\
\hline & S06029 & symbiotic & V.sativa \\
\hline & S09031 & symbiotic & T. repens \\
\hline & S09077 & symbiotic & V.sativa \\
\hline & S09109 & symbiotic & T. repens \\
\hline & S09112 & symbiotic & T. repens \\
\hline & S09113 & symbiotic & T. repens \\
\hline & S09133 & symbiotic & T. repens \\
\hline & S11078 & symbiotic & V.sativa \\
\hline
\end{tabular}


Supplementary Table 2.S2. Exclusively selected genes for each host-selected subpopulation $(1 / 4)$

\begin{tabular}{|c|c|c|}
\hline \multicolumn{3}{|l|}{ Pea } \\
\hline Product & Gene & \\
\hline $\begin{array}{l}\text { (3R)-hydroxymyristoyl-ACP dehydratase } \\
\text { (fabZ) }\end{array}$ & RL2230 & \\
\hline $\begin{array}{l}\text { 3-oxoacyl-(acyl carrier protein) synthase II } \\
\text { (fbF2) }\end{array}$ & RL2815 & \\
\hline 4'-phosphopantetheinyl transferase (acpS) & RL1509 & \\
\hline acetyltransferase & RL2077 & RL2804 \\
\hline ATP synthase I (atpl) & RL0924 & \\
\hline F0F1 ATP synthase subunit B (atpF) & RL0928 & \\
\hline cyclopropane-fatty-acyl-phospholipid synthase & RL1982 & \\
\hline deoxygenase $(c f a)$ & RL0802 & \\
\hline DGPFAETKE domain-containing protein & RL1476 & \\
\hline dihydrodipicolinate reductase $(\mathrm{dap} B)$ & RL3002 & \\
\hline DNA-3-methyladenine glycosylase I (tag) & RL0871 & \\
\hline glucose-1-phosphate thymidylyltransferase & RL0795 & \\
\hline glutathione S-transferase (gstB) & RL1284 & \\
\hline $\begin{array}{l}\text { hexapeptide repeat-containing } \\
\text { acetyltransferase }\end{array}$ & RL0823 & \\
\hline hexapeptide repeat-containing transferase & RL0799 & \\
\hline $\begin{array}{l}\text { imidazole glycerol phosphate synthase } \\
\text { subunit }(\text { hisH) }\end{array}$ & RL0820 & RL0819 \\
\hline NADH oxidase & RL2502 & \\
\hline $\begin{array}{l}\text { nicotinic acid mononucleotide } \\
\text { adenylyltransferase }(\text { nadD })\end{array}$ & RL4684 & \\
\hline oxidoreductase & RL0824 & \\
\hline phosphoribosyl-ATP pyrophosphatase (hisE) & RL0041 & \\
\hline phosphoribosyltransferase & RL3870 & \\
\hline phosphoserine phosphatase (serB) & RL3250 & \\
\hline phosphotransferase & RL3115 & \\
\hline protease & RL1858 & \\
\hline putative acetyltransferase & pRL110463 & \\
\hline putative alcohol dehydrogenase ( $\operatorname{adhA}$ ) & pRL110134 & pRL90027 \\
\hline putative amidohydrolase & pRL110027 & \\
\hline hydroxypyruvate reductase & RL0994 & \\
\hline putative beta-mannosidase & pRL110397 & \\
\hline putative CobW family protein & pRL110127 & \\
\hline putative creatinine amidohydrolase & pRL120303 & \\
\hline putative dehydrogenase/oxidoreductase & pRL120530 & \\
\hline putative desulfurization/monoxygenase & pRL90300 & \\
\hline putative dihydrodipicolinate synthase & pRL110028 & pRL120528 \\
\hline putative FAD/NAD/ferredoxin protein & pRL120537 & \\
\hline putative fixS protein & pRL90012A & \\
\hline putative flavonol synthase/dioxygenase & pRL110611 & \\
\hline putative FMN oxidoreductase & pRL110496 & \\
\hline $\begin{array}{l}\text { putative glucosamine--fructose-6-phosphate } \\
\text { aminotransferase [isomerizing] }\end{array}$ & pRL120250 & \\
\hline putative glycosyl hydrolase & pRL120559 & \\
\hline putative glycosyltransferase & pRL110390 & \\
\hline putative haloalkane dehalogenase & pRL110536 & \\
\hline putative hydrogenase protein $(h y c D)$ & pRL110296 & \\
\hline putative hydrogenase- 4 component (hyfFE) & pRL110294 & pRL110295 \\
\hline
\end{tabular}


Supplementary Table 2.S2. Exclusively selected genes for each subpopulation (2/4)

\begin{tabular}{|c|c|c|c|}
\hline Pea & & & \\
\hline Product & Gene & & \\
\hline $\begin{array}{l}\text { putative nitrilase/cyanide hydratase family } \\
\text { protein }\end{array}$ & pRL120440 & & \\
\hline $\begin{array}{l}\text { putative nitrilase/cyanide hydratase family } \\
\text { protein }\end{array}$ & pRL120440 & & \\
\hline putative nucleotide binding protein & pRL120407 & & \\
\hline $\begin{array}{l}\text { putative oxygen-independent } \\
\text { coproporphyrinogen III oxidase }\end{array}$ & pRL90023 & & \\
\hline $\begin{array}{l}\text { putative phosphatidylinositol alpha- } \\
\text { mannosyltransferase }\end{array}$ & pRL110391 & & \\
\hline putative phosphoesterase/regulator & pRL110368 & & \\
\hline putative proline racemase & pRL120535 & & \\
\hline putative transmembrane nitrile hydratase & pRL110123 & & \\
\hline putative sarcosine oxidase delta subunit & pRL110561 & & \\
\hline $\begin{array}{l}\text { putative two-component regulator response } \\
\text { regulator protein }\end{array}$ & pRL90026 & & \\
\hline putative universal stress protein & pRL90047 & & \\
\hline rhizobiocin & RL0790 & & \\
\hline shikimate dehydrogenase (aroE) & RL2845 & & \\
\hline short-chain dehydrogenase & RL1981 & & \\
\hline short-chain dehydrogenase/reductase & RL1814 & & \\
\hline Smr domain-containing protein & RL0010 & & \\
\hline septum site-determining protein MinC & pRL110546 & & \\
\hline $\begin{array}{l}\text { succinate dehydrogenase cytochrome b556 } \\
\text { subunit }(s d h C)\end{array}$ & RL4446 & & \\
\hline tartrate dehydrogenase & RL0995 & & \\
\hline $\begin{array}{l}\text { UDP-N-acetylmuramoyl-L-alanyl-D-glutamate } \\
\text { synthetase (murD) }\end{array}$ & RL3309 & & \\
\hline universal stress related protein & RL1868 & & \\
\hline ArsR family transcriptional regulator & RL4000 & & \\
\hline putative nucleotide binding protein & pRL120407 & & \\
\hline AsnC family transcriptional regulator & RL1861 & pRL80067 & \\
\hline $\begin{array}{l}\text { bifunctional autolysin AtIE/transcriptional } \\
\text { regulator AtIR }\end{array}$ & RL1320 & & \\
\hline GntR family transcriptional regulator & RL4419 & pRL110208 & pRL120527 \\
\hline HTH family transcriptional regulator & RL1214 & & \\
\hline LysR family transcriptional regulator & RL2848 & & \\
\hline putative AraC family transcriptional regulator & pRL110119 & pRL120536 & pRL120257 \\
\hline putative Lacl family transcriptional regulator & pRL120618 & & \\
\hline putative LysR family transcriptional regulator & $\begin{array}{l}\text { pRL110026 } \\
\text { pRL80061 }\end{array}$ & pRL120410 & pRL100331 \\
\hline $\begin{array}{l}\text { putative MerR family heavy metal dependent } \\
\text { transcriptional regulator }\end{array}$ & pRL110330 & & \\
\hline putative transcriptional regulator & pRL120447 & pRL80056A & \\
\hline $\begin{array}{l}\text { putative transcriptional regulator adenylate } \\
\text { cyclase }\end{array}$ & pRL110112 & & \\
\hline TetR family transcriptional regulator & RL1233 & & \\
\hline lipopolysaccharide synthesis protein & RL0818 & & \\
\hline O-antigen transporter & RL0821 & & \\
\hline OmpA family outer membrane protein & RL3267 & & \\
\hline outer membrane transport protein & RL2036 & & \\
\hline polysaccharide biosynthesis protein & RL3654 & & \\
\hline
\end{tabular}


Supplementary Table 2.S2. Exclusively selected genes for each subpopulation (3/4)

\begin{tabular}{|c|c|c|c|}
\hline \multicolumn{4}{|l|}{ Pea } \\
\hline Product & Gene & & \\
\hline $\begin{array}{l}\text { UTP--glucose-1-phosphate uridylyltransferase } \\
\text { (pss } N)\end{array}$ & RL4081 & & \\
\hline \multirow{3}{*}{$\begin{array}{l}\text { putative ATP-binding component of ABC } \\
\text { transporter } \\
\text { putative permease component of } A B C \\
\text { transporter }\end{array}$} & $\begin{array}{l}\text { pRL100463 } \\
\text { pRL120532 }\end{array}$ & pRL110021 & pRL120430 \\
\hline & pRL120443 & pRL120444 & pRL120533 \\
\hline & pRL120534 & & \\
\hline \multirow{9}{*}{$\begin{array}{l}\text { putative substrate-binding component of } A B C \\
\text { transporter } \\
\text { solute-binding component of } A B C \text { transporter } \\
\text { transmembrane component of } A B C \\
\text { transporter } \\
\text { putative efflux transporter involved in } \\
\text { vicibactin siderophore export }(v b s D) \\
\text { putative exopolysaccharide production protein } \\
\text { putative exported peptidase } \\
\text { putative exported solute-binding protein } \\
\text { putative lipid biosynthesis 3-oxoacyl-[acyl- } \\
\text { carrier-protein] reductase } \\
\text { putative transmembrane protein }\end{array}$} & pRL120445 & pRL120531 & \\
\hline & RL3842 & & \\
\hline & RL1583 & & \\
\hline & pRL120317 & & \\
\hline & pRL110389 & & \\
\hline & pRL110597 & & \\
\hline & pRL80060 & & \\
\hline & pRL100460 & & \\
\hline & $\begin{array}{l}\text { pRL110116 } \\
\text { pRL110431 }\end{array}$ & $\begin{array}{l}\text { pRL110392 } \\
\text { pRL110596 }\end{array}$ & pRL110396 \\
\hline \multirow{4}{*}{$\begin{array}{l}\text { putative transmembrane TIM family protein } \\
\text { transmembrane glycosyl transferase } \\
\text { transmembrane protein }\end{array}$} & $\begin{array}{l}\text { pRL90272 } \\
\text { RL0801 }\end{array}$ & & \\
\hline & RL0538 & RL0636 & RL0797 \\
\hline & RL0817 & RL1281 & RL1392 \\
\hline & RL2071 & RL2081 & RL3117 \\
\hline \multirow{2}{*}{$\begin{array}{l}\text { transmembrane protein } \\
\text { chaperone protein }\end{array}$} & RL3186 & & \\
\hline & RL2565 & & \\
\hline \multirow{2}{*}{$\begin{array}{l}\text { ECF RNA polymerase sigma- } 70 \text { family } \\
\text { putative } 30 \text { S ribosomal protein }\end{array}$} & RL4524 & & \\
\hline & pRL100450 & & \\
\hline conjugal transfer protein (traA) & RL0772 & & \\
\hline \multirow{2}{*}{$\begin{array}{l}\text { invasion-associated protein } \\
\text { putative flagellar hook protein flgE }\end{array}$} & RL2390 & & \\
\hline & pRL120064 & & \\
\hline \multirow{2}{*}{$\begin{array}{l}\text { putative plasmid stability protein } \mathrm{StbC} \\
\text { putative RepB replication protein }\end{array}$} & pRL120075 & & \\
\hline & pRL100002 & & \\
\hline insertion sequence related protein & RL2330 & & \\
\hline \multirow{2}{*}{$\begin{array}{l}\text { putative insertion sequence protein } \\
\text { transposase }\end{array}$} & pRL70144 & & \\
\hline & RL0457 & & \\
\hline \multirow{9}{*}{$\begin{array}{l}\text { conserved hypothetical exported protein } \\
\text { conserved hypothetical protein }\end{array}$} & pRL110107 & & \\
\hline & pRL100190 & pRL100191 & pRL100234 \\
\hline & pRL100329 & pRL110020 & pRL110083 \\
\hline & pRL110092 & pRL110093 & pRL110120 \\
\hline & pRL110194 & pRL110209 & pRL110217 \\
\hline & pRL110268 & pRL110328 & pRL120293 \\
\hline & pRL120371 & pRL120654 & pRL90022 \\
\hline & pRL90032 & pRL90048 & pRL90040 \\
\hline & pRL90042 & & \\
\hline \multirow[t]{3}{*}{ hypothetical protein } & pRL110317A & pRL110326 & pRL110398 \\
\hline & pRL110399 & pRL110426 & pRL110497 \\
\hline & pRL120211 & pRL120512 & pRL120656 \\
\hline
\end{tabular}


Supplementary Table 2.S2. Exclusively selected genes for each subpopulation (4/4)

\begin{tabular}{|c|c|c|c|}
\hline \multicolumn{4}{|l|}{ Pea } \\
\hline Product & Gene & & \\
\hline \multirow[t]{14}{*}{ hypothetical protein } & pRL70006 & pRL90029 & pRL90046 \\
\hline & pRL90241 & RL0014 & RL0034 \\
\hline & RL0070 & RL0154 & RL0193 \\
\hline & RL0330 & RL0773 & RL0795A \\
\hline & RL0800 & RL0803 & RL0867 \\
\hline & RL0957 & RL1039 & RL1094 \\
\hline & RL1116 & RL1278 & RL1430 \\
\hline & RL1480 & RL1677 & RL1768 \\
\hline & RL2309 & RL2765 & RL2858 \\
\hline & RL2862 & RL2874 & RL2955 \\
\hline & RL3005 & RL3054 & RL3360 \\
\hline & RL3412 & RL3493 & RL3987 \\
\hline & RL4005 & RL4112 & RL4347 \\
\hline & RL4656 & & \\
\hline No description & pRL90313 & & \\
\hline \multicolumn{4}{|l|}{ Lentil } \\
\hline Product & Gene & & \\
\hline putative oxidoreductase & pRL110139 & & \\
\hline acetyl transferase (wbbJ) & RL0241A & & \\
\hline \multicolumn{4}{|l|}{ Fava bean } \\
\hline Product & Gene & & \\
\hline $\begin{array}{l}\text { putative virulence-island/protein involved in } \\
\text { nitrogen fixation (impE) } \\
\text { conserved hvpothetical protein involved in }\end{array}$ & pRL120471 & & \\
\hline $\begin{array}{l}\text { conserved hypothetical protein Involved in } \\
\text { nitrogen fixation (impD) }\end{array}$ & pRL120472 & & \\
\hline $\begin{array}{l}\text { putative virulence-ISlana/proteln Involved in } \\
\text { nitrogen fixation (impC) } \\
\text { putative ImcF family transmembrane protein }\end{array}$ & pRL120473 & & \\
\hline $\begin{array}{l}\text { putative ImcF family transmembrane protein } \\
\text { involved in nitrogen fixation ( } \mathrm{impL} \text { ) }\end{array}$ & pRL120464 & & \\
\hline putative conjugal transfer protein (traDp7) & pRL70084 & & \\
\hline $\begin{array}{l}\text { putative DNA conjugal transfer protein } \\
\text { (traCp7) }\end{array}$ & pRL70085 & & \\
\hline \multicolumn{4}{|l|}{ Vetch } \\
\hline Product & Gene & & \\
\hline hypothetical protein & RL2133 & & \\
\hline
\end{tabular}



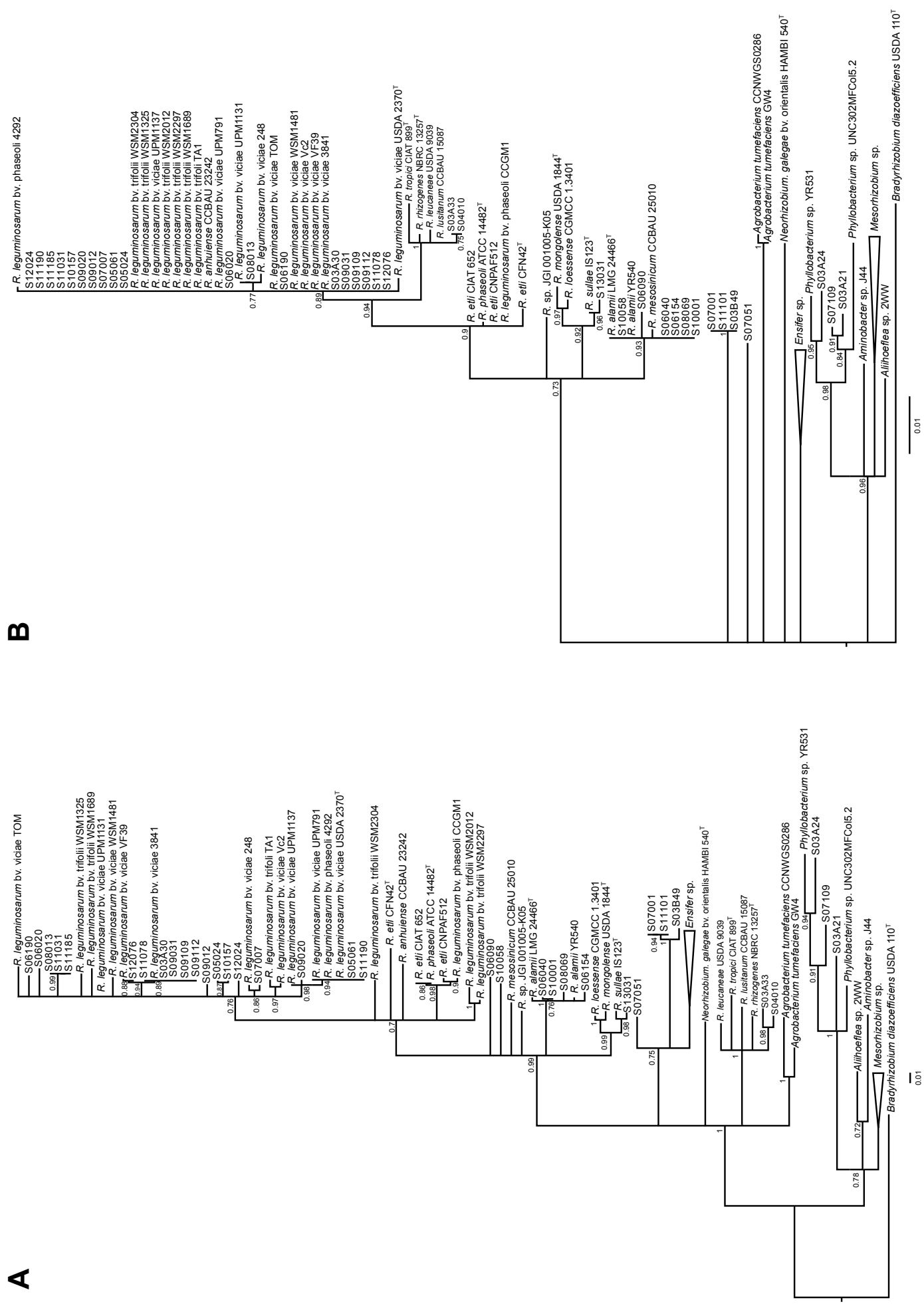

Figure 2.S1. Phylogenetic tree based on partial (447 nt) rpoB (A) and near complete $(1,287 \mathrm{nt}) 16 \mathrm{rRNA}$ sequences showing the relationship of selected P1 soil isolates and related species within the order Rhizobiales. The tree was constructed by the neighborjoining method (A) and by the maximum likelihood method (B). The significance of each branch is indicated by a bootstrap value calculated for 1,000 subsets. Nodes with bootstrap values lower than 0.7 were collapsed. Bar represents 0.01 substitutions. The rpoB and 16S rRNA sequence of Bradyrhizobium diazoefficiens USDA $110^{\top}$ was used as outgroup. Abbreviations: R. Rhizobium. 

6. CHAPTER 3. Mesocosm Analysis of Rhizobium leguminosarum Genotype Selection by Different Fabeae Legume Hosts 



\subsection{INTRODUCTION}

In legume-rhizobial symbioses that form indeterminate nodules, those rhizobia that differentiate into mature bacteroids do so irreversibly, and thus forswear reproduction (Sprent et al., 1987). As a result, only undifferentiated bacteria that persisted in infection threads are liberated and contribute to replenish the rhizobial population in the soil (Vance et al., 1980, Paau et al., 1980). Timmers et al. (2000) defined a specific saprophytic zone $V$ in alfalfa senescent nodules; this region presented vegetative bacteria in the intracellular spaces and in the lumen of central cells of the most proximal part of indeterminate nodules (after zone IV). Both regions have the capacity to reintegrate viable rhizobia into soil after plant tissue disintegration. It has been proposed, and generally accepted, that symbiosis enhances the reproductive success of rhizobia because the increase in rhizobial numbers after nodule senescence is significantly higher than the increment that would occur through microbial multiplication in soil (Sprent et al., 1993, Denison and Kiers, 2004).

There are numerous reports of rhizobial population fluctuations in response to different crops, and over time. For instance Bradyrhizobium japonicum is able to persist in soil during years in the absence of its host; in contrast Ensifer meliloti was very sensitive to crop rotations (Triplett et al., 1993). Rhizobium leguminosarum inoculants were sensitive to long-term mono-cropping practises with non-host plants (Kucey and Hynes, 1989). Moreover, pea plant cultivation increased soil $R$. leguminosarum bv. viciae populations ten times more than cereals or beans (Kucey and Hynes, 1989). Similarly, $R$. leguminosarum bv. trifolii appeared to be ten times more abundant in pastures where clover was present (Nutman and Hearne, 1979). Despite these observations, little is known on how crops and time affect microbial diversity in soil. For instance, populations of arbuscular mychorrizal fungi had much higher diversity in woodland fields than in arable samples (Helgason et al., 1998). Unsurprisingly, observations regarding rhizobia differ widely, and are dependent on factors such as plant type, bacterial species, soil type, climate, and agronomic practices. As an example, the cultivation of the host plant has been shown to have a homogenizing effect on rhizobial populations (Hirsch, 1996). Handley et al. (1998) compared the $R$. leguminosarum bv. viciae diversity in arable fields and in an uncultivated roadside, and also found that diversity is not affected by cultivation per se. This is in line with previous observations that a similar genetic diversity was found for $R$. leguminosarum bv. viciae populations isolated from grazed pastures and woodland, despite different densities, different plant communities and the presence or absence of the local host (Strain et al., 1994). However, in a more recent study, Palmer and Young (2000) showed a 
significantly higher number of $R$. leguminosarum types in arable sites than in a grassland. A lower rhizobial diversity in a wild population, as compared to that found in cultivated fields, has also been observed for Rhizobium etli (Souza and Eguiarte, 1997). Although little is known about factors affecting rhizobial diversity in soil environments, it is widely acknowledged that the plant community present can be a relevant parameter. For instance, $R$. leguminosarum diversity was 100 times lower in maize mono-cropping than in wheat (Depret et al., 2004). It has been suggested that the presence and age of alfalfa plants in a cultivated field have an effect on the diversity of the native $R$. leguminosarum bv. viciae population, which is dominated by one or two genotypes (Zhang et al., 2001). Many of these investigations seem to indicate that rhizobial diversity may be higher in agricultural situations than in wild populations, but this evidence is far from conclusive (Mutch and Young, 2004).

It has been proposed that some Rhizobium leguminosarum genotypes could be more adapted to specific plant genera, species or cultivars (Davis et al., 1988, Bromfield et al., 1995, Hartmann et al., 1998, Zeze et al., 2001, Laguerre et al., 2003, Mutch and Young, 2004). We have shown that legume hosts can exhibit a preference for specific rhizobial genotypes out of those present in the soil, and that this selection is dependent on the legume species (Chapter 1) (Jorrín and Imperial, 2015b); this selection is not the same for the different plant species used in this Thesis. In Chapter 2 we were able to show that pea plants selected a significantly different population from that present in the soil, whereas lentil and fava bean plants reflected in their nodules the population present in soil. However, it is not known whether this selection pattern will be maintained along multiple plant infection cycles.

Given that plants can differentially select rhizobial genotypes and that viable rhizobia of those genotypes are released into soil after nodule senescence, we can hypothesize that, in natural conditions after numerous cycles of selection-release most nodules should be occupied by the preferred genotype/s in each plant. In the present Chapter we have experimentally tested this hypothesis in a mesocosm experiment aimed at mimicking these field conditions. 


\subsection{RESULTS}

In the present Chapter we have analysed the continuous selection effect in a control system in order to mimic natural conditions. On one side a Pool-Seq analysis was performed in order to compare the results obtained in the mesocosm experiment with the previous Pool-Seqs (initial host and soil). As well a genomic analysis of mesocosm-selected strains was conducted to confirm the results obtained in the PoolSeq analysis. The genomic analysis was restricted to the preliminary conclusions obtained in the Pool-Seq analysis. A complete comparative genomic analysis is conducting, but the results are not in the scope of the present Thesis.

\subsubsection{Pool-Seq analysis}

A Pool-Seq approach was used in order to ascertain the effect of the continuous selection effect, as well as compare the results with the previous Pool-Seqs.

\subsubsection{Mesocosm-selected rhizobial populations}

Mesocosm-selected $R$. leguminosarum bv. viciae populations consisted of twentyfive isolates obtained, one each, from surface-sterilized root nodules excised from trap plants obtained after five cycles of culture. Isolates were plate-purified, re-tested for symbiotic properties, and stored. Eight populations were obtained: pea $_{A}$ and pea $_{B}$ (Pisum sativum), lentil $\mathrm{A}_{\mathrm{A}}$ and lentil $\mathrm{B}_{\mathrm{B}}$ (Lens culinaris), vetch $_{\mathrm{A}}$ and vetch $_{\mathrm{B}}$ (Vicia sativa) and

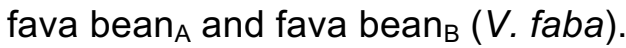

\subsubsection{Next generation sequencing of pooled, mesocosm plant-selected rhizobial populations}

DNAs from pooled samples of each of the eight different mesocosm $R$. leguminosarum bv. viciae subpopulations were submitted to next-generation sequencing (Pool-Seq) so that, on average, each of the twenty-five genomes was sequenced to $1-4 x$ coverage. (Illumina MiSeq V3, 600 bp PE libraries, 300 bp reads, 1 Mreads, ASU Genomics Core, Tempe, AZ for pea $\mathrm{A}_{\mathrm{A}}$ for the remaining Pool-Seqs Illumina MiSeq V2, 500bp PE libraries, 250 bp reads, 1-2 Mreads, Microseq Enterprise, Las Vegas, NV). Individual sequencing reads from each mesocosm subpopulation were recruited to the reference genome (Materials and Methods, (Jorrín and Imperial, $2015 \mathrm{~b}$ ) with the maximum fragment length option (-X) of Bowtie2 incremented to 2,000 in view of both the dimension of libraries size and the chemistry of synthesis. Coverage results were normalized to Base Per Kilobase per Million reads (BPKM, (Yang et al., 
2011)) to correct for differences in sequencing depth and read length among different Pool-Seq datasets (including plant-selected Pool-Seqs from Chapter 1 and soil PoolSeq form Chapter 2).

6.2.1.3. Presence of sequences from the R. leguminosarum bv. viciae 3841 reference genome in mesocosm plant-selected rhizobial populations

The $R$. leguminosarum bv. viciae 3841 reference genome is multipartite and contains six large plasmids : pRL7 (151.5 kb) through pRL12 (870 kb), in addition to a $5,057 \mathrm{~kb}$ circular chromosome (Young et al., 2006). The presence and conservation of sequences from these seven replicons in the mesocosm plant-selected Pool-Seq dataset was investigated (Table 3.1). The small plasmids ( $p R L 7$ and $p R L 8$ ) were minor components in all the mesocosm subpopulations; pRL7 was absent (BPKM lower than 10 ) in fava bean ${ }_{A}$ and vetch $_{B}$, and nearly absent (BPKM between 10 and 25) in lentil $A_{A}$, lentil ${ }_{B}$ and fava bean ${ }_{B}$. However, in pea $_{A}$, pea $_{B}$ and vetch ${ }_{A}$, genes present in pRL7 were enriched during the experiment. In pea $_{A}$ and pea $_{B}$ the average coverage (31.7 and 51.99, BPKM respectively) was more than two-fold higher than the average coverage in host Pool-Seqs after 1 cycle (6.04-7.82 BPKM; Table 3.1, Figure 3.4). These data suggest that genes present in this plasmid were enriched during the mesocosm experiment due to the effect of pea plant. On the other hand, pRL8 was absent or nearly absent in all mesocosm plant-selected subpopulations with the exception of pea $_{\mathrm{A}}$, where the average coverage was $40.56 \mathrm{BPKM}$, more than two-fold higher than the average coverage in host Pool-Seqs after 1 cycle (6.3-7.25 BPKM; Table 3.1 and Figure 3.3). It is noteworthy that in fava bean ${ }_{B}$ there was a decrease in the average

Table 3.1. Average coverage (BPKM) of each reference genome (Rhizobium leguminosarum bv. viciae 3841) replicon by all Pool-Seq sample datasets.

\begin{tabular}{cccccccc}
\hline & Chromosome & $\mathrm{pRL7}$ & $\mathrm{pRL8}$ & $\mathrm{pRL9}$ & $\mathrm{pRL10}$ & $\mathrm{pRL11}$ & $\mathrm{pRL12}$ \\
\hline Pea $_{\mathrm{A}}$ & 92.37 & 32.70 & 40.56 & 88.22 & 65.57 & 72.94 & 88.55 \\
Pea $_{\mathrm{B}}$ & 117.21 & 51.99 & 2.92 & 123.32 & 73.94 & 82.27 & 108.00 \\
Lentil $_{\mathrm{A}}$ & 110.03 & 20.02 & 19.99 & 118.28 & 101.09 & 87.94 & 107.37 \\
Lentil $_{\mathrm{B}}$ & 113.91 & 12.98 & 6.64 & 108.18 & 87.66 & 93.79 & 102.84 \\
Fava bean $_{\mathrm{A}}$ & 108.33 & 7.25 & 3.62 & 99.49 & 86.25 & 88.17 & 95.64 \\
Fava bean $_{\mathrm{B}}$ & 106.79 & 10.34 & 1.58 & 98.60 & 88.73 & 87.67 & 92.29 \\
Vetch $_{\mathrm{A}}$ & 111.76 & 25.77 & 19.55 & 110.88 & 76.97 & 87.92 & 103.62 \\
Vetch $_{\mathrm{B}}$ & 92.32 & 5.54 & 3.27 & 58.93 & 74.45 & 71.21 & 77.21 \\
\hline Pea & 118.14 & 7.82 & 7.25 & 93.07 & 75.14 & 87.53 & 107.73 \\
Lentil $_{\text {Fava bean }}$ & 112.09 & 6.04 & 6.44 & 84.60 & 75.24 & 78.77 & 97.76 \\
Vetch & 107.85 & 6.92 & 7.02 & 97.06 & 87.88 & 84.21 & 98.55 \\
\hline Soil & 119.34 & 6.46 & 6.30 & 84.02 & 76.01 & 80.03 & 100.09 \\
\hline
\end{tabular}


coverage of pRL8 (1.58 BPKM), ca. two-fold lower as compared to host values (Table 3.1 and Figure 3.3). In the majority of the selected subpopulations the chromosome and plasmids pRL12 and pRL9 showed the highest average coverage values, followed by $p R L 11$ and $p R L 10$, with the exception of vetch ${ }_{B}$, where $p R L 9$ showed an average coverage value of 58.93 BPKM, thus recruiting about half the reads recruited by the remaining mesocosm subpopulations (88.22 to 123.32 BPKM).

Coverage (BPKM) plots along reference genome replicon sequences are presented in Figures 3.1 to 3.4 for chromosome, pRL10, pRL8 and pRL7 and Supplementary Figures $3 . S 1$ to $3 . S 3$ for $p R L 12, p R L 11$ and pRL9. Numerous differences were found among replicons in the defined low-coverage regions (spanning more than 40 genes) and in overrepresented peaks (above 200 BPKM) (Jorrín and Imperial, 2015b):

i) Regarding the chromosome (Figure 3.1), low-coverage region 1 (RL0791RL0841), region 3 (RL2105-RL2195) and region 4 (RL3912-RL3956), were also present in the mesocosm Pool-Seqs (Figure 3.1, panels $A-H$ ); however region 2 (RL1869-RL1944) was only present in pea $_{A}$, fava bean ${ }_{B}$ and vetch $_{B}$. Differences in overrepresented peaks were found in the chromosome: peak a (RL2002), was only maintained in pea $_{\mathrm{B}}$ and disappeared in the remaining mesocosm Pool-Seqs. New overrepresented peaks (over 200 BPKM) were defined: $C_{a}$ (RL0074, hypothetical protein), $\mathrm{Ch}_{\mathrm{b}}$ (RL1487, hypothetical protein), $\mathrm{Ch}_{\mathrm{c}}$ (RL1916, hypothetical protein), $\mathrm{Ch}_{\mathrm{d}}$ (RL2452-RL2453, hypothetical protein and transposase-related protein), $\mathrm{Ch}_{\mathrm{e}}(\mathrm{RL} 3549$, glnll, glutamine synthetase), $\mathrm{Ch}_{\mathrm{f}}(4,086,001-4,091,000), \mathrm{Ch}_{\mathrm{g}}$ (RL4249-RL4250, ATPbinding and transmembrane protein from CUT1-type $A B C$ transporter) and ${ } h_{h}$ $(4,544,001-4,546,000)$ and $\mathrm{Ch}_{\mathrm{i}}$ (RL4655, rhizopine-binding $\mathrm{ABC}$ transporter protein) (Table 3.2). These peaks were only found in pea $_{A}$, pea $a_{B}$, fava bean ${ }_{A}$ and vetch $_{B}$ mesocosm subpopulations. Peak $\mathrm{Ch}_{\mathrm{g}}$ (RL4249-4250), was located in both pea mesocosm subpopulations $\left(\mathrm{pea}_{\mathrm{A}}\right.$ and pea $_{\mathrm{B}}$ ), which suggests that these genes were enriched in pea nodules after five cycles of selection.

Table 3.2. Presence (grey) and absence (white) of overrepresented chromosome peaks for each mesocosm host-selected subpopulation.

\begin{tabular}{|c|c|c|c|c|c|c|c|c|}
\hline & $\mathrm{Pea}_{\mathrm{A}}$ & $\mathrm{Pea}_{\mathrm{B}}$ & Lentil $_{A}$ & Lentil $_{B}$ & Fava bean ${ }_{A}$ & Fava bean $_{B}$ & Vetch $_{\mathrm{A}}$ & Vetch $_{B}$ \\
\hline \multicolumn{9}{|l|}{$a$} \\
\hline \multicolumn{9}{|l|}{$\mathrm{Ch}_{\mathrm{a}}$} \\
\hline \multicolumn{9}{|c|}{$\mathrm{Ch}_{\mathrm{b}}$} \\
\hline \multicolumn{9}{|l|}{$\mathrm{Ch}_{\mathrm{c}}$} \\
\hline \multicolumn{9}{|l|}{$\mathrm{Ch}_{\mathrm{d}}$} \\
\hline \multicolumn{9}{|l|}{$\mathrm{Ch}_{\mathrm{e}}$} \\
\hline \multicolumn{9}{|c|}{$\mathrm{Ch}_{\mathrm{f}}$} \\
\hline \multicolumn{9}{|l|}{$\mathrm{Ch}_{\mathrm{g}}$} \\
\hline \multicolumn{9}{|l|}{$\mathrm{Ch}_{\mathrm{h}}$} \\
\hline $\mathrm{Ch}_{\mathrm{i}}$ & & & & & & & & \\
\hline
\end{tabular}





Figure 3.1. Conservation of the chromosome of $R$. leguminosarum bv. viciae 3841 reference genome in the mesocosm plant-selected subpopulations. Coverage (BPKM) of genome regions along the chromosome by Bowtie2 recruited reads from $\operatorname{pea}_{A}(A), \operatorname{pea}_{B}(B)$, lentil $(C)$, lentil $(D)$, fava bean $A(E)$, fava bean $(F), \operatorname{vetch}_{A}(G)$, vetch ${ }_{B}(\mathrm{H})$ and host $(\mathrm{I})$. "Host" is defined as the average of initial plant-selected subpopulations from Chapter 1 (Figure 1.1). Colour-coding of the different hosts: red (pea), blue (lentil), green (fava bean) and purple (vetch) has been maintained throughout. The location and span of 4 low $\mathrm{G}+\mathrm{C}$ islands described by (Young et al., 2006) in the reference genome are indicated by grey bars at the bottom. Major under-represented regions in the mesocosm plant-selected datasets are numbered, while over-represented regions are named $\mathrm{Ch}_{\mathrm{a}-\text { through }} \mathrm{Ch}_{\mathrm{h}}$. 
ii) Supplementary Figure 3.S1 shows plots of coverage along pRL12. The defined low-coverage region 5 (pRL120440-pRL120479) that includes a T6SS, was present in all mesocosm subpopulations with the exception of pea $a_{A}$. Two new low-coverage regions were observed: $p R L 12_{1}$ (pRL120102-pRL120183) and $p R L 12_{2}$ (pRL120561pRL120604), spanning 82 and 44 genes, respectively. These were genes of different annotated functions, including enzymes, transcriptional factors, transmembrane and transporter proteins, a tra system $\left(\mathrm{pRL} 12_{2}\right)$ and hypothetical proteins. Region $\mathrm{pRL} 12_{2}$ was only present in vetch $\mathrm{B}_{\mathrm{B}}$, while $\mathrm{pRL} 12_{1}$ was present in fava bean $\mathrm{n}_{\mathrm{B}}$ and partially in vetch $_{B}$, where just pRL120135-pRL120155, pRL120165 and pRL120166 were present. Two overrepresented peaks were also defined: $p R L 12{ }_{a}$ (pRL120521, etfB2, putative electron-transfer flavoprotein ß-unit) and $p R L 12_{b}$ (pRL120646, transposase-related protein), which appeared in pea $_{A}$ and vetch ${ }_{B}$, respectively.

iii) In plasmid pRL11 the low-coverage region 6 (pRL110145-pRL110188) was maintained during the mesocosm experiment (Supplementary Figure 3.S2). In some cases, this region was extended to $\mathrm{pRL} 110112$ ( vetch $_{B}$ and pea $_{B}$ ), or even to pRL110094 (fava B $^{\text {). }}$

iv) The symbiotic plasmid ( $p R L 10$, Figure 3.2) showed the highest differences compared with initial host-selected population (panel I). Low-coverage region 7 (pRL100011-pRL100138) was only maintained per se in fava bean and lentil mesocosm subpopulations, while in vetch and pea, some of the genes located in region 7 were present or even overrepresented. Therefore we had defined two new low coverage regions $p R L 10_{1}$ (pRL100012-pRL100038) in pea $a_{A}$ and pea $a_{B}$; and pRL10 (pRL100012-pRL100074) in vetch ${ }_{A}$ and vetch ${ }_{B}$. New overrepresented peaks appeared in the symbiotic plasmid: pRL10a (pRL100078-pRL100081, qat6VW), pRL10 (pRL100094, no description), $p R L 10_{c}$ (pRL100190, hypothetical protein) and $p R L 10_{d}$ (pRL100206, fixO). Peaks pRL10 and pRL10 $10_{d}$, were only detected in pea mesocosm subpopulations, whereas $p R L 10_{b}$ and $p R L 10_{c}$ were exclusive for pea $a_{B}$ and fava bean $_{B}$ respectively.

v) Plasmid pRL9 was not conserved in vetch ${ }_{B}$, where the average coverage was lower compared with the remaining mesocosm Pool-Seqs (Table 3.1), and this was reflected in the coverage along the replicon (Supplementary Figure 3.S3, panel H), where numerous low-covered regions were detected. The overrepresented peak $b$ (pRL90159) was spotted in pea $_{A}$, pea ${ }_{B}$, lentil ${ }_{B}$ and fava bean ${ }_{A}$. In lentil ${ }_{A}$ the BPKM value in this region was of 168 and in fava bean $_{B}$, of 197; although they did not exceed the 200 BPKM limit, coverages of peak $b$ were the highest values found for pRL9. A new overrepresented peak was defined for pea mesocosm Pool-Seqs, pRL $9_{a}$ (pRL90013-pRL90014, fixl and fixH). 


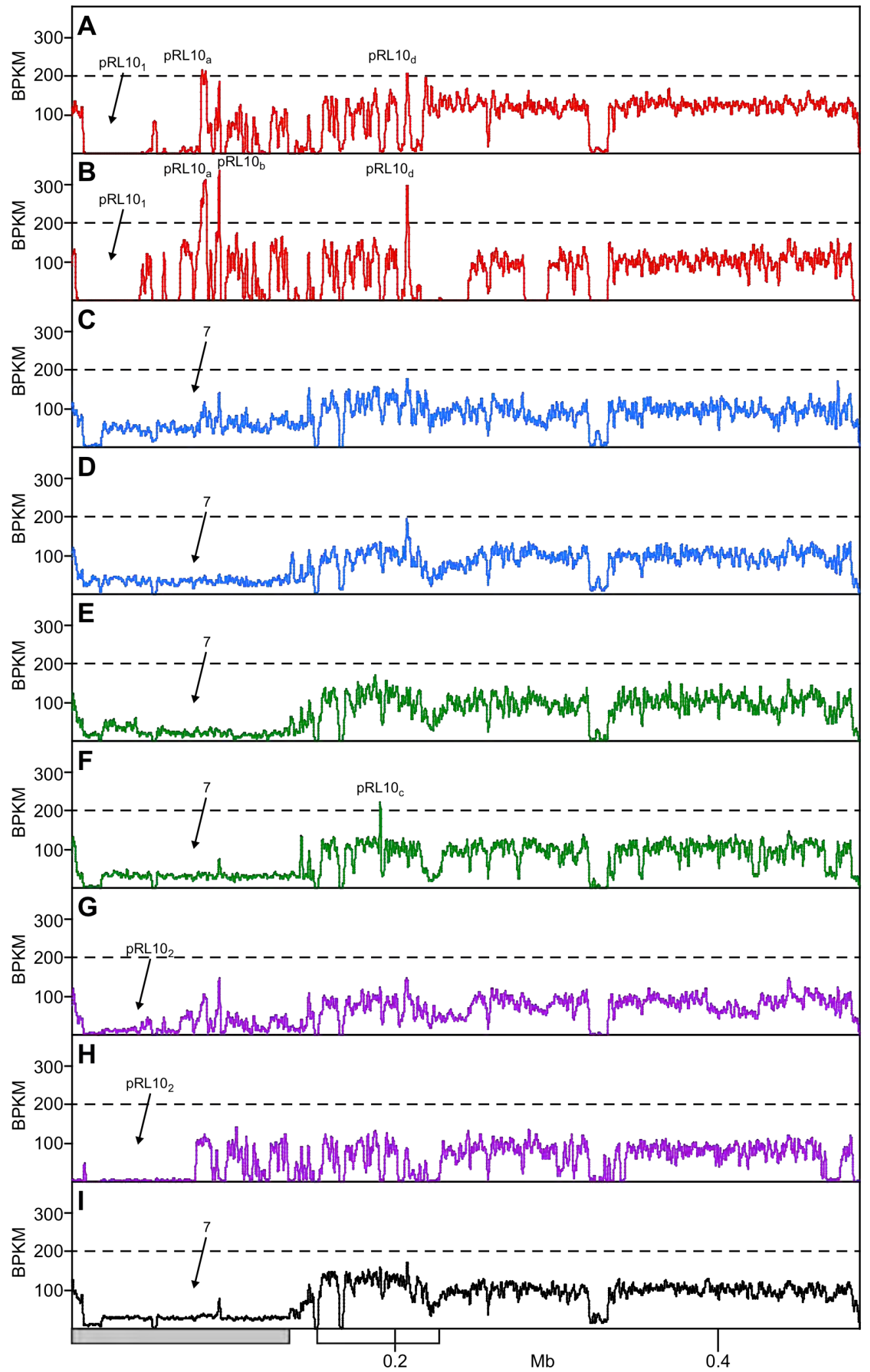

Figure 3.2. Conservation of the symbiotic plasmid pRL10 of $R$. leguminosarum bv. viciae 3841 reference genome in the mesocosm plant-selected subpopulations. Coverage (BPKM) of genome regions along $\mathrm{pRL} 10$ by Bowtie2 recruited reads from $\operatorname{pea}_{A}(A), \operatorname{pea}_{B}(B)$, lentil $(C)$, lentil $B_{B}(D)$, fava bean $A(E)$, fava bean $B(F)$, vetch $(G)$, vetch ${ }_{B}(\mathrm{H})$ and host $(\mathrm{I})$. The location and span of the low $\mathrm{G}+\mathrm{C}$ island described by (Young et al., 2006) in the reference genome is indicated by a grey bar at the bottom. The symbiotic region is indicated by a white bar. Major under- and over-represented regions in the mesocosm plant-selected datasets are indicated numerically or as $\mathrm{pRL} 10_{1-2}$, and as $\mathrm{pRL} 10_{\mathrm{a}-\mathrm{d}}$, respectively. 
vi) Despite the lower average coverage values found for small plasmids ( $p R L 7$ and pRL8), large differences were uncovered among mesocosm Pool-Seqs. Plasmid pRL8 was nearly absent in all mesocosm host-selected subpopulations (Figure 3.3). The

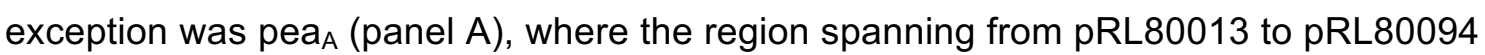
was present, although the overrepresented peak pRL8 ${ }_{a}$ (pRL80057, hypothetical protein) could be detected throughout. On the other hand, pRL7 also showed differences among mesocosm subpopulations (Figure 3.4). $\mathrm{Pea}_{\mathrm{A}}$ and $\mathrm{pea}_{\mathrm{B}}$ clearly selected some of genes present in this plasmid and new overrepresented peaks were appeared: pRL7 ${ }_{a}$ (pRL70067, putative transposase) only present in pea $a_{B} ; p R L 7_{b}$ (pRL70152-pRL70153, crown gall-like T4SS protein and hypothetical protein, respectively), present in pea $_{\mathrm{A}}$ and pea $_{\mathrm{B}}$; and pRL7 $\mathrm{c}$ (pRL70155-pRL70158, traJGL and virB11, respectively) exclusive for pea . 


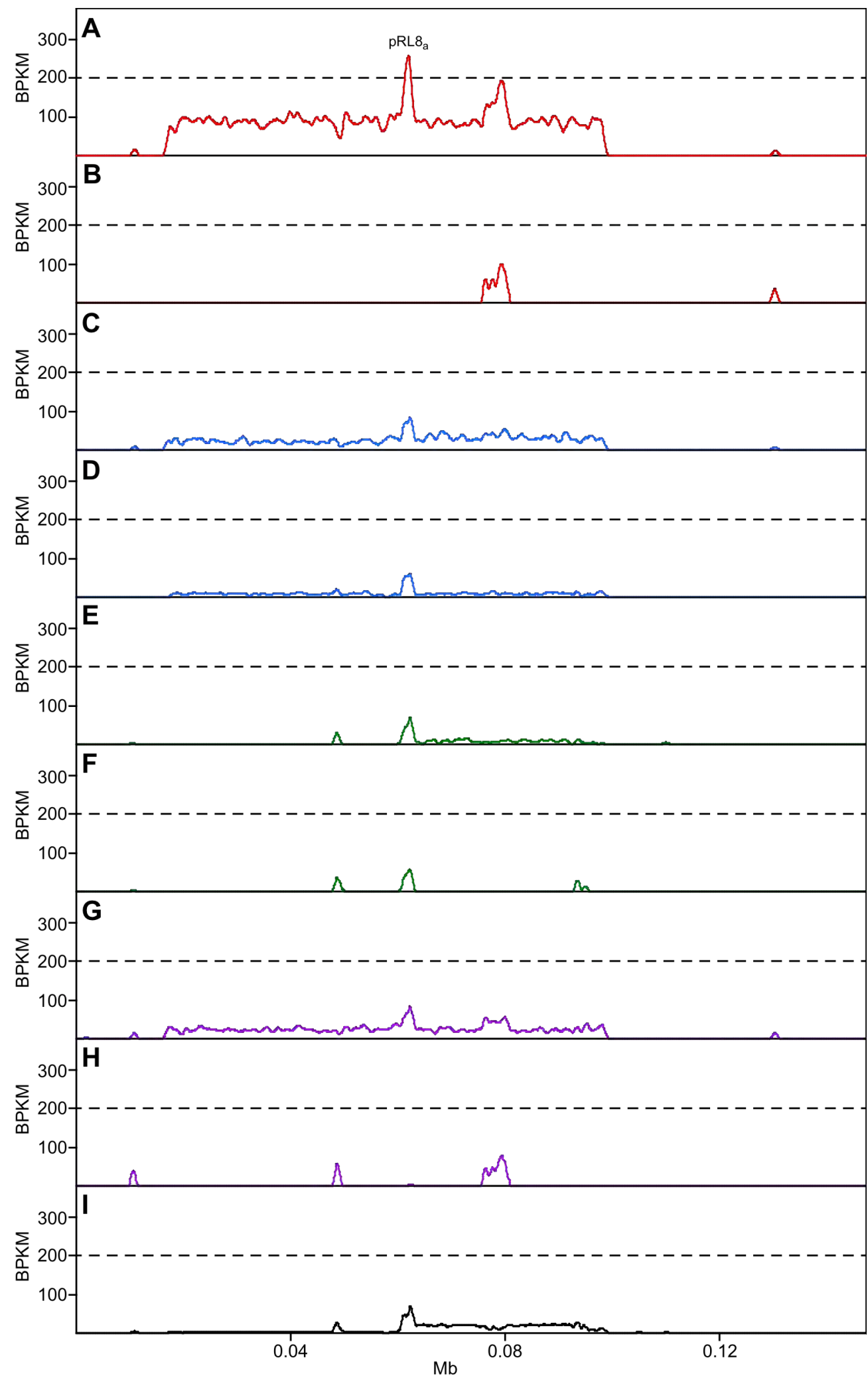

Figure 3.3. Conservation of the plasmid pRL8 of $R$. leguminosarum bv. viciae 3841 reference genome in the mesocosm plant-selected subpopulations. Coverage (BPKM) of genome regions along pRL8 by Bowtie2 recruited reads from pea $(A)$,

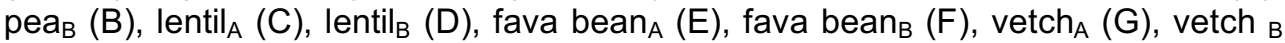
$(\mathrm{H})$ and host $(\mathrm{I})$. A major over-represented region in a mesocosm plant-selected dataset is indicated as pRL8a. 


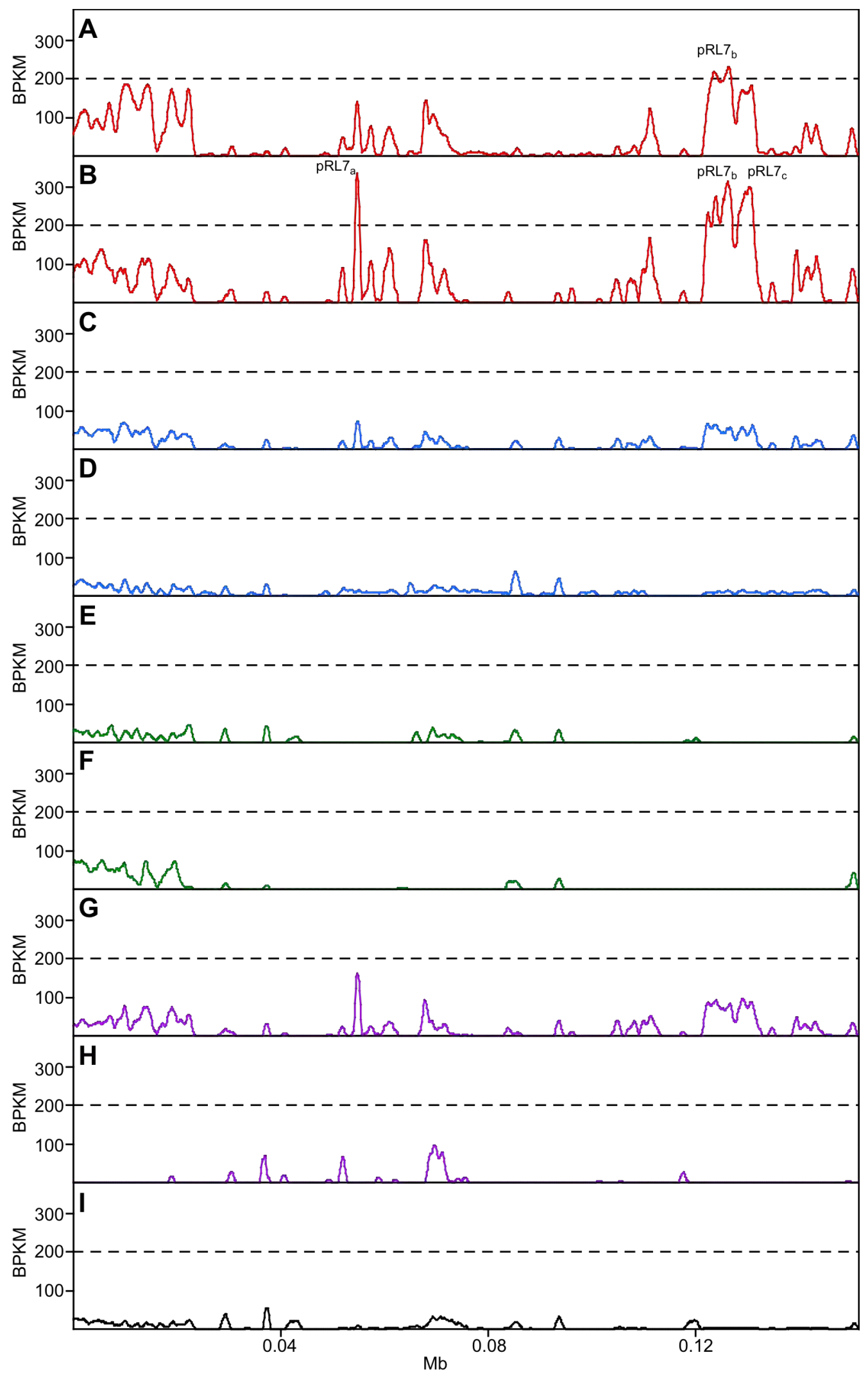

Figure 3.4. Conservation of the plasmid pRL7 of $R$. leguminosarum bv. viciae 3841 reference genome in the mesocosm plant-selected subpopulations. Coverage (BPKM) of genome regions along pRL7 by Bowtie2 recruited reads from pea $(A)$, pea $_{B}(B)$, lentil $_{A}(C)$, lentil $B(D)$, fava bean $(E)$, fava $\operatorname{bean}_{B}(F)$, $\operatorname{vetch}_{A}(G)$, vetch $B(H)$ and host (I). Major over-represented regions in the mesocosm plant-selected datasets are indicated as $\mathrm{pRL} 7_{\mathrm{a}-\mathrm{c}}$. 
6.2.1.4. Conservation of genes from the $R$. leguminosarum bv. viciae 3841 reference genome in plant host-selected subpopulations.

Due to the observed coverage differences among mesocosm subpopulations (see above) and initial plant-selected subpopulations (see Chapter 1), a comparative coverage analysis was performed within and between each host subpopulations. For each predicted gene, BPKM values were calculated, and a threshold was defined to operationally consider whether a gene is conserved or absent, or whether it is overrepresented. These thresholds were as follows: conserved, above 10 BPKM; absent, below 10 BPKM; and overrepresented, above 200 BPKM. Comparisons among plant host subpopulations were plotted as Venn diagrams.

\subsection{Conserved genes}

Figure 3.5 shows results for conserved genes, where pea, lentil and fava bean subpopulations had a core value above 6,200 genes, while the vetch subpopulation presented a core value below 5,500 genes that undoubtedly reflected the low recruitment values for vetch $\mathrm{B}_{\mathrm{B}}$. Panel $\mathrm{E}$ summarizes the distribution of conserved genes among the different host-selected populations. Strikingly, very few genes were found to be present exclusively in each host-selected population, except for lentils (see also Supplementary Table S1). Among the twenty four genes that were exclusively present in the three pea-selected subpopulations (initial and both mesocosm replicates), the presence of tra and trb genes from plasmid pRL7 stands out. Lentil Pool-Seqs showed the highest number of exclusive genes (one hundred and one); among them it is worth noting the presence of three members of the imp cluster (T6SS), despite the fact that this cluster is located in pRL12 low-coverage region 5 (pRL120440-pRL120479), and suggest that the T6SS was maintained at low coverage during the mesocosm experiment in lentil. Only four genes were exclusive for fava bean-selected subpopulations, all of them located in the chromosome and annotated as three hypothetical proteins and RLt23 (tRNA-Glu). Vetch subpopulations showed just two exclusive genes, an arylsulfatase (RL1911) localized in the chromosome, and a transposase from pRL11 (pRL110352). 


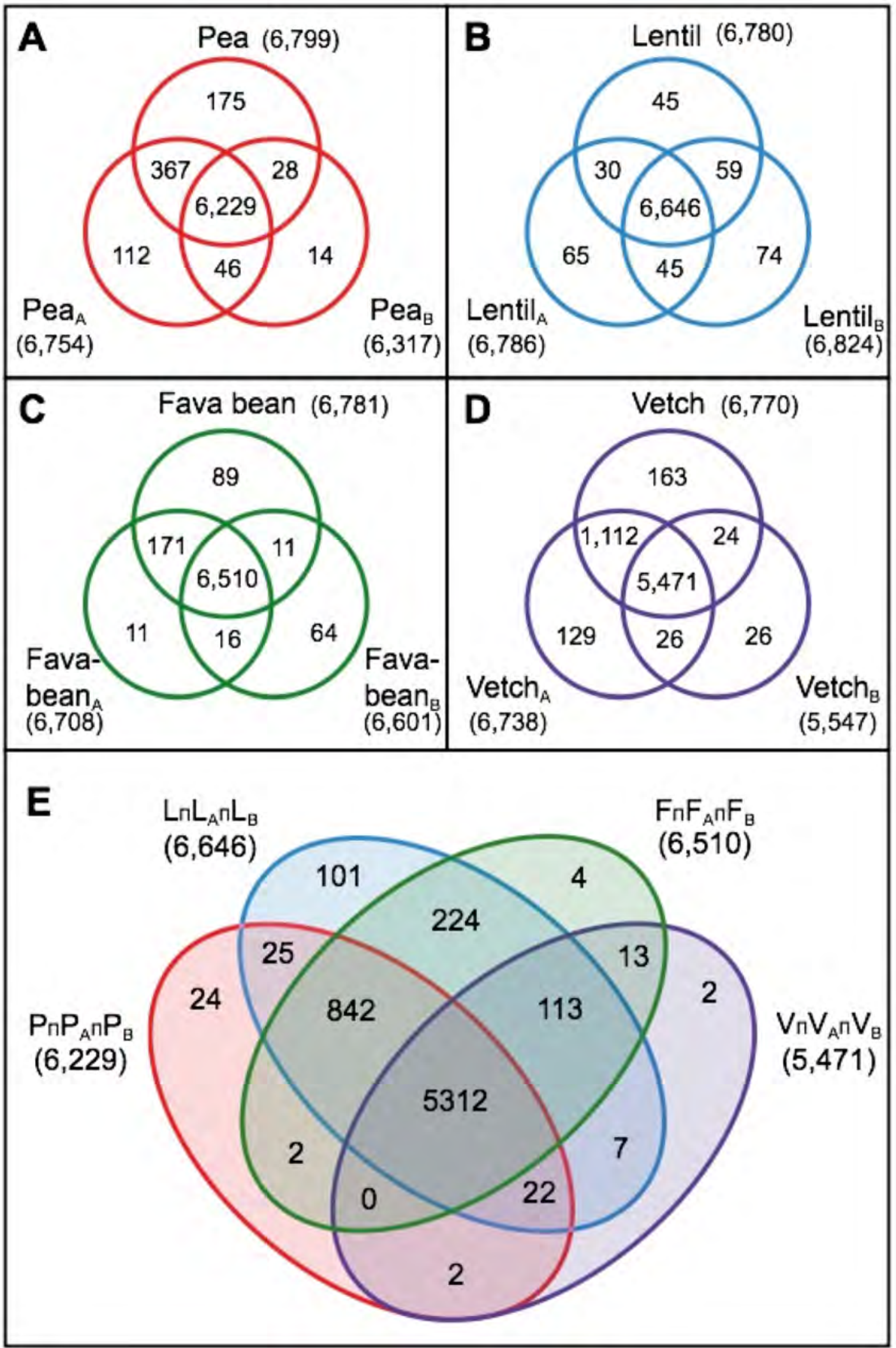

Figure 3.5. Venn diagrams of conserved genes among host-selected subpopulations. The intersections of host-selected subpopulations are plotted in panels $A$ (initial pea $(P)$, pea $\left(P_{A}\right)$ and pea $a_{B}\left(P_{B}\right)$ ), $B$ (initial lentil $(L)$, lentil $A_{A}\left(L_{A}\right)$ and lentil $l_{B}\left(L_{B}\right)$ ), $C$ (initial fava bean $(F)$, fava bean $\mathrm{b}_{A}\left(\mathrm{~F}_{\mathrm{A}}\right)$ and fava bean $\left.\mathrm{B}_{B}\left(\mathrm{~F}_{\mathrm{B}}\right)\right)$ and $\mathrm{D}$ (initial vetch $(\mathrm{V})$, vetch $\mathrm{v}_{\mathrm{A}}\left(\mathrm{V}_{\mathrm{A}}\right)$ and vetch $_{B}\left(\mathrm{~V}_{B}\right)$ ). The intersection of core conserved genes among host-selected subpopulations is plotted in panel E. Numbers of genes in each subpopulation and in the different intersections are indicated. 


\subsection{Absent genes}

All host-selected rhizobial subpopulations presented similar values for core absent genes: between 308-329 for pea, lentil and vetch, and 408 for fava bean subpopulations (Figure 3.6). Panel E summarizes the distribution of absent genes among the different host-selected populations. Except for the fava bean population, that contained 98 exclusively absent genes, the core values were similar, between 1516 genes, in the remaining host-selected subpopulations (pea, lentil and vetch). These genes are listed in Supplementary Table S2. For the pea-selected subpopulation, most of the absent genes $(10 / 16)$ belong to the beginning of low coverage region $\mathrm{PRL} 10_{1}$. Most of lentil-selected subpopulation absent genes were chromosomal (8/16) or pRL11 (6 / 16) genes. For the fava bean-selected subpopulation, most of the absent genes were located in the small plasmids (37 and 48 in pRL7 and pRL8 respectively, out of 98). Relevant among them was the absence of a carbon monoxide dehydrogenase system ( $p R L 80021-p R L 80025)$, a branched-chain ABC transporter (IivJMHGF, pRL80026-pRL80030), and a poly-ß-hydroxybutyrate synthesis protein (phnA, pRL100320), as well as genes related with tra and trb system of pRL7. Finally, for vetch Pool-Seqs most absent genes were pRL11 and pRL7 genes (9 and 5, respectively). 


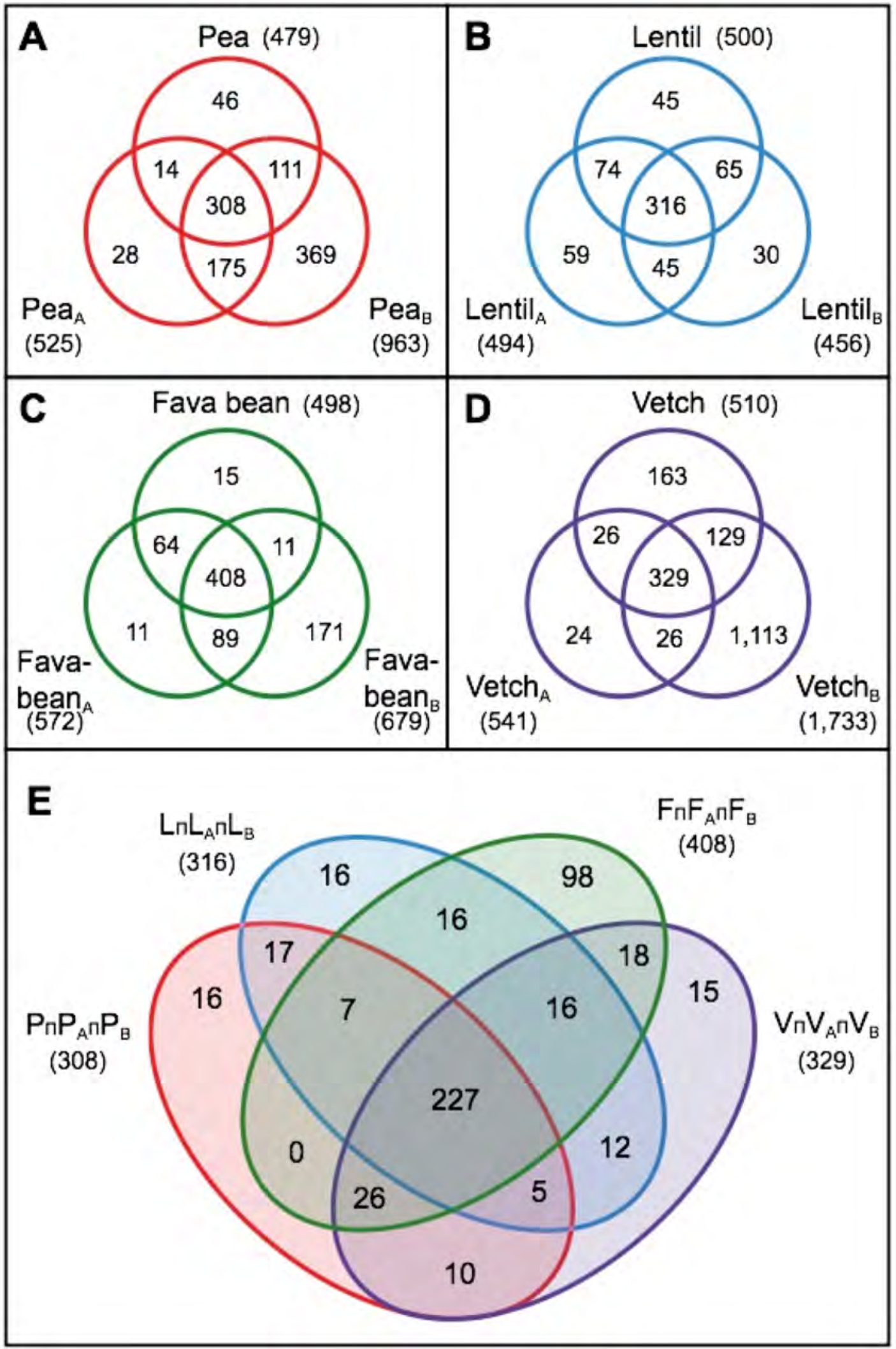

Figure 3.6. Venn diagrams of absent genes among host-selected subpopulations. The intersections of host-selected subpopulations are plotted in panels $A$ (initial pea $(P)$, pea $\left(P_{A}\right)$ and pea $\left(P_{B}\right)$ ), $B$ (initial lentil $(L)$, lentil $A_{A}\left(L_{A}\right)$ and lentil $l_{B}\left(L_{B}\right)$ ), $C$ (initial fava bean $(F)$,

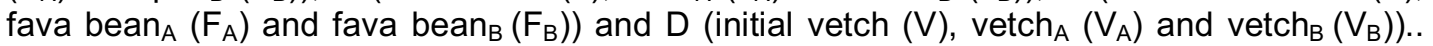
The intersection of core absent genes among host-selected subpopulations is plotted in panel E. Numbers of genes in each subpopulation and in the different intersections are indicated. 


\subsection{Overrepresented genes}

A low number of CDS from the reference genome were present and overrepresented in all the host-selected subpopulations. After five cycles, nine genes were enriched in pea mesocosm Pool-Seqs (peaA and peaB): RL4250, pRL100079, pRL100081 and pRL100082 (all of them components of ABC transporters), pRL100206 (fixO), pRL70152-pRL70153 (virB4-virB5) and pRL90013-pRL90014 (fixlfixH). Additionally, one gene (RL2002, transposase-related protein) was overrepresented in both initial pea and pea ${ }_{\mathrm{B}}$ Pool-Seqs. Similarly, two hypothetical protein-encoding genes (pRL100190 and pRL100191) were overrepresented when comparing fava bean and fava bean ${ }_{B}$ (Figure 3.7).

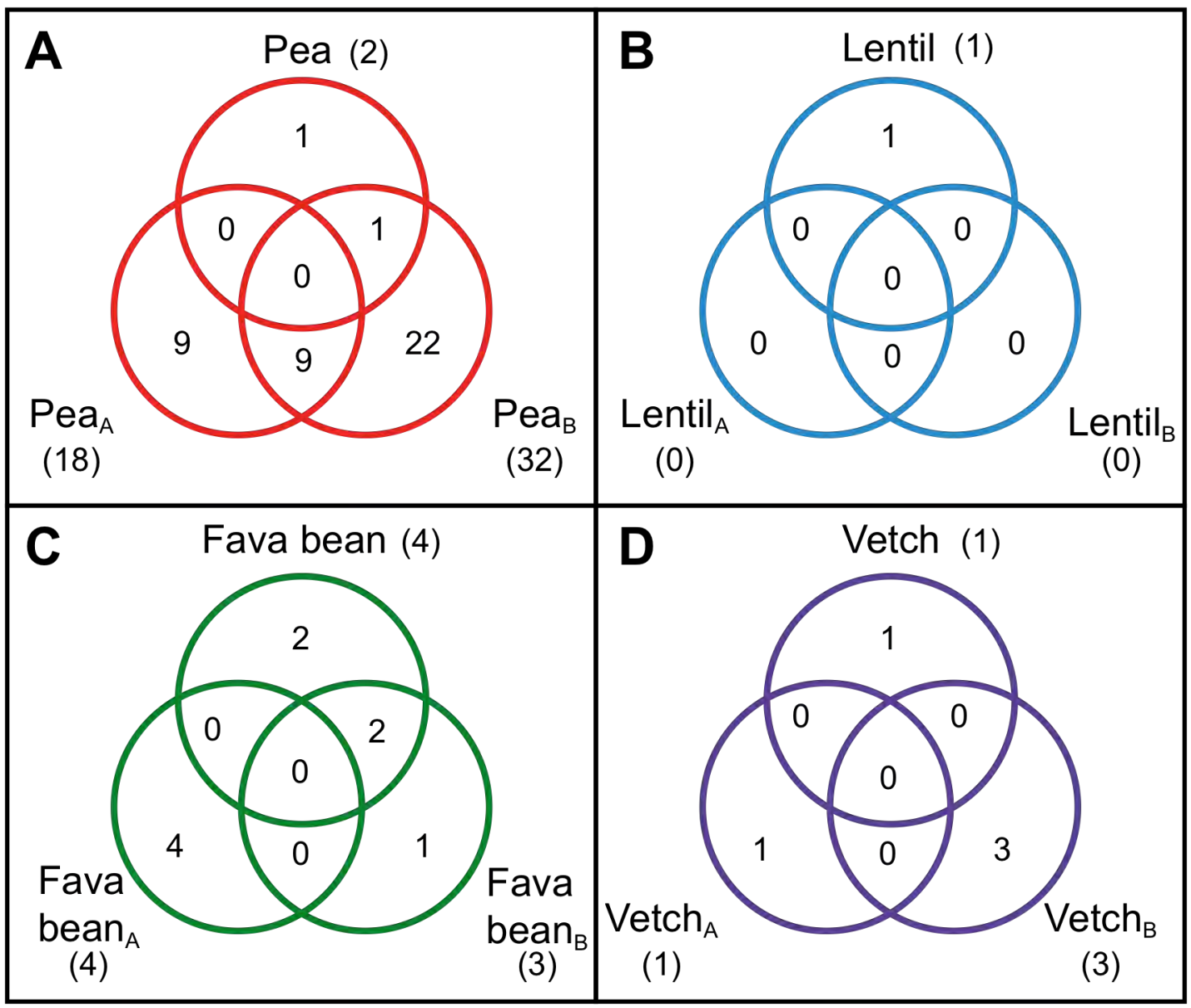

Figure 3.7. Venn diagrams of overrepresented genes for each host-selected subpopulation. The intersections of host-selected subpopulations are plotted in panels $A$ (initial pea $(P)$, pea $\left(P_{A}\right)$ and pea ${ }_{B}\left(P_{B}\right)$ ), $B$ (initial lentil $(L)$, lentil $A_{A}\left(L_{A}\right)$ and lentil ${ }_{B}\left(L_{B}\right)$ ), $C$ (initial fava bean $(F)$, fava bean $_{A}\left(F_{A}\right)$ and fava bean ${ }_{B}\left(F_{B}\right)$ ) and $D$ (initial vetch $(V)$, vetch ${ }_{A}\left(V_{A}\right)$ and vetch $_{B}\left(V_{B}\right)$ ).. Numbers of genes in each subpopulation and in the different intersections are indicated. 
6.2.1.5. Differential coverage of $R$. leguminosarum bv. viciae 3841 reference genome sequences in host-selected Pool-Seqs

A comparison coverage analysis was performed between the initial host-selected subpopulations (Chapter 1) and mesocosm Pool-Seqs, in order to investigate differential gene presence after five cycles of plant-host selection. No CDS had been found to be significantly different among the initial Pool-Seqs (pea, lentil, fava bean and vetch; Chapter 1); therefore, an average BPKM was calculated and defined as "initial host." For each gene predicted in the reference genome $R$. leguminosarum bv. viciae $3841, \log _{2}$ values of fold-change were calculated to compare each mesocosm hostselected Pool-Seq with the average initial host-selected BPKMs. Genes with $\log _{2}$ foldchange greater than 2 , or lower than -2 , were considered to be more abundant in mesocosm or initial host-selected Pool-Seqs, respectively. Table 3.3 summarizes these results, and Venn diagrams were constructed for each set of enriched and depleted genes within and among host-selected populations (Figure 3.8 and 3.9, respectively). Overall, a larger number of genes was decreased after five cycles of host-selection.

Table 3.3. Number of genes that were significantly different between each mesocosm PoolSeq and the average of initial host Pool-Seqs

\begin{tabular}{|c|c|c|}
\hline & Enriched & Depleted \\
\hline$P e a_{A}$ & 208 & 218 \\
\hline $\mathrm{Pea}_{\mathrm{B}}$ & 114 & 758 \\
\hline Lentil $_{\mathrm{A}}$ & 108 & 188 \\
\hline Lentil $_{B}$ & 63 & 64 \\
\hline Fava bean ${ }_{A}$ & 6 & 272 \\
\hline Fava bean ${ }_{B}$ & 39 & 496 \\
\hline Vetch $_{\mathrm{A}}$ & 115 & 230 \\
\hline Vetch $_{B}$ & 70 & 1,703 \\
\hline
\end{tabular}

\subsection{Genes exclusively enriched after five cycles of host selection}

Different numbers of genes were found to be enriched for each host-selected subpopulation (Figure 3.8). Pea showed the highest core value (73), followed by lentil (27), vetch (15) and fava bean (2). When all enriched genes within each host-selected subpopulation were cross-compared among all host subpopulations, only one gene was enriched in all mesocosm Pool-Seqs as compared to initial host: RL1191, a transmembrane protein located in the chromosome (Figure 3.8 panel E). The above relative abundance order was maintained for exclusively enriched genes for each subpopulation (52, 12, 3 and 1 for pea, lentil, vetch and fava bean, respectively) and 


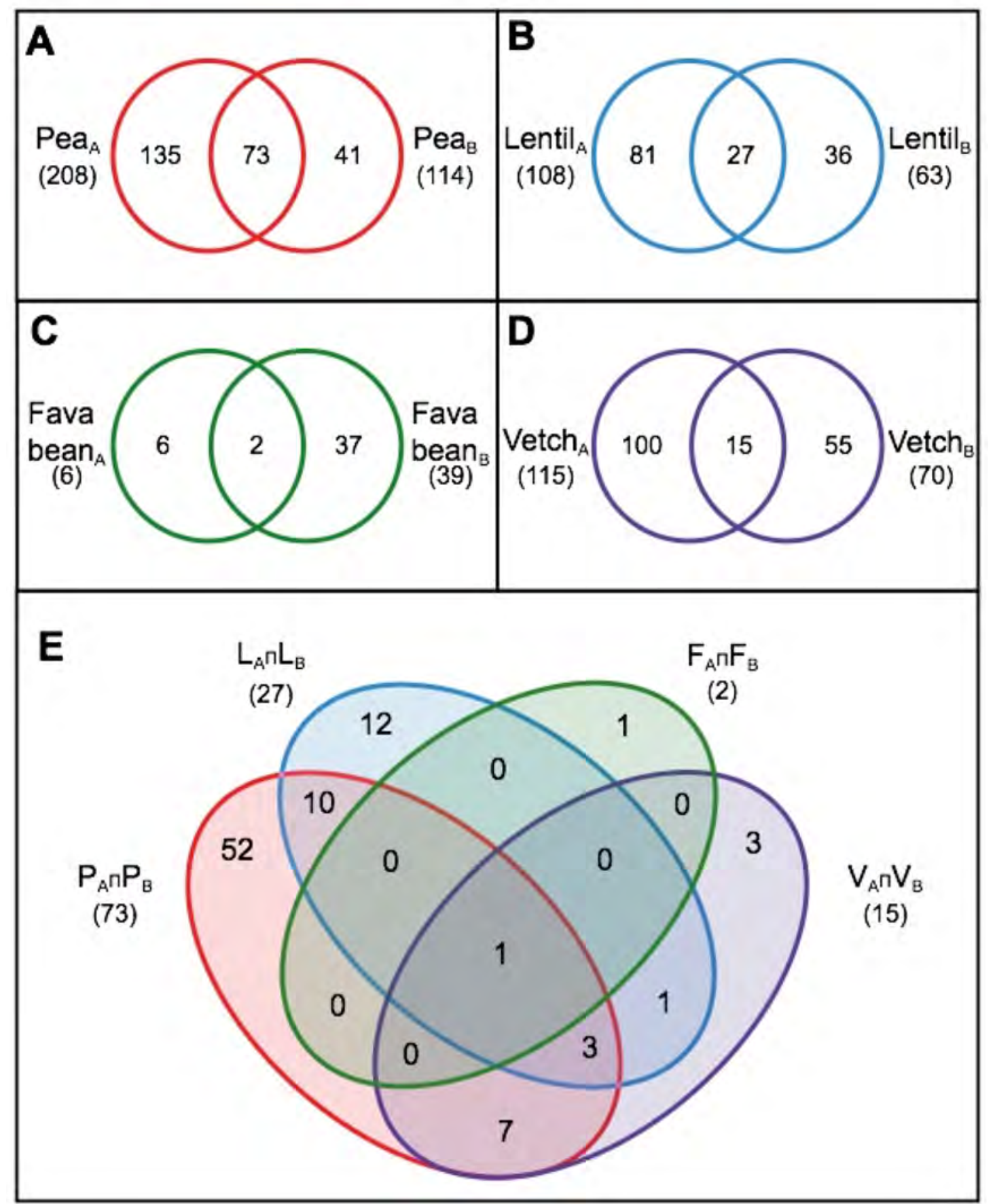

Figure 3.8. Venn diagrams of genes exclusively enriched after five cycles of host selection among mesocosm host-selected subpopulations. Each mesocosm host-selected subpopulation was compared with the initial host-selected subpopulation. $\log _{2}$ values of foldchange were calculated and genes with $\log _{2}$ (fold-change) $>2$ were considered to be more abundant in mesocosm host-selected Pool-Seqs. The intersections of host-selected subpopulations are plotted in panels $A\left(\right.$ pea $_{A}\left(P_{A}\right)$ and pea $\left(P_{B}\right)$ ), $B$ (lentil $\left.\right|_{A}\left(L_{A}\right)$ and lentil ${ }_{B}$ $\left(L_{B}\right)$ ), $C$ (fava bean ${ }_{A}\left(F_{A}\right)$ and fava bean $\left.{ }_{B}\left(F_{B}\right)\right)$ and $D\left(\operatorname{vetch}_{A}\left(V_{A}\right)\right.$ and vetch $B\left(V_{B}\right)$ ). The intersection of core absent genes among mesocosm host-selected subpopulations is plotted in panel E. Numbers of genes in each subpopulation and in the different intersections are indicated. 
these genes are listed in Supplementary Table S3. The tra and trb pRL7 genes that were found to be present in the three pea-selected subpopulations (Supplementary Table S1) were also enriched during the mesocosm experiment. It is noteworthy that a quaternary ammonium $\mathrm{ABC}$ transporter (qat6) was exclusively enriched in peaselected mesocosm subpopulations. In lentil mesocosm Pool-Seqs, twelve genes were enriched after five cycles of host selection, and the majority were located in pRL8 (10 / 12). Only one gene was exclusively enriched in fava bean mesocosm subpopulations, a hypothetical protein-encoding gene located in the chromosome. Likewise, in vetch-selected mesocosm subpopulations only three genes were enriched, all encoding hypothetical proteins and located, two in the chromosome and one in pRL12.

\subsection{Genes exclusively depleted after five cycles of host-selection}

Different depleted genes core values were found for each mesocosm host-selected subpopulations (Figure 3.9). Fava bean subpopulations showed the highest core value (201), followed by vetch (192), pea (176) and lentil (40). When depleted genes within each mesocosm host-selected subpopulations were compared among all hosts, only one gene was decreased in all mesocosm Pool-Seqs compared to initial host, namely pRL70145, a putative transposase-related protein located in pRL7 (Figure 3.9, panel E). The above relative abundance order was not maintained for exclusively depleted genes $(117,104,67$ and 7 for fava bean, pea, vetch and lentil, respectively), and these genes are listed in Supplementary Table S4. In pea mesocosm Pool-Seqs most depleted genes were located in the symbiotic plasmid (pRL10, 68 out of 104). It is noteworthy that symbiosis-related genes, such as $f d x B$, fixl, fixS and fixH, from pRL10, appeared to be depleted after five cycles of host-selection together with genes related to qat4 and qat5 $\mathrm{ABC}$ transporter and an acetyl-coenzyme A synthetase (acs $A 3)$. Only 7 genes were depleted after five cycles of lentil selection: a DNA polymerase II subunit $(d n a E)$, together with hypothetical protein-encoding genes, a transmembrane protein gene and a mobile element. Fava bean showed the highest number of depleted exclusive genes (117), and these were localized, mainly in the small plasmids (53 and 41 genes in $\mathrm{pRL7}$ and $\mathrm{pRL8}$, respectively). The carbon monoxide dehydrogenase (pRL80021-pRL80025), the branched-chain ABC transporter (livJMHGF, pRL80026pRL80030), as well as genes related with tra and trb system of pRL7 and the poly-ßhydroxybutyrate synthesis protein (phnA, pRL100320) had been previously assigned as "absent" in fava bean Pool-Seqs (see 6.2.1.4.2 above). Even so, reads mapping to these genes were further depleted after five cycles of selection. Finally, in the vetch 


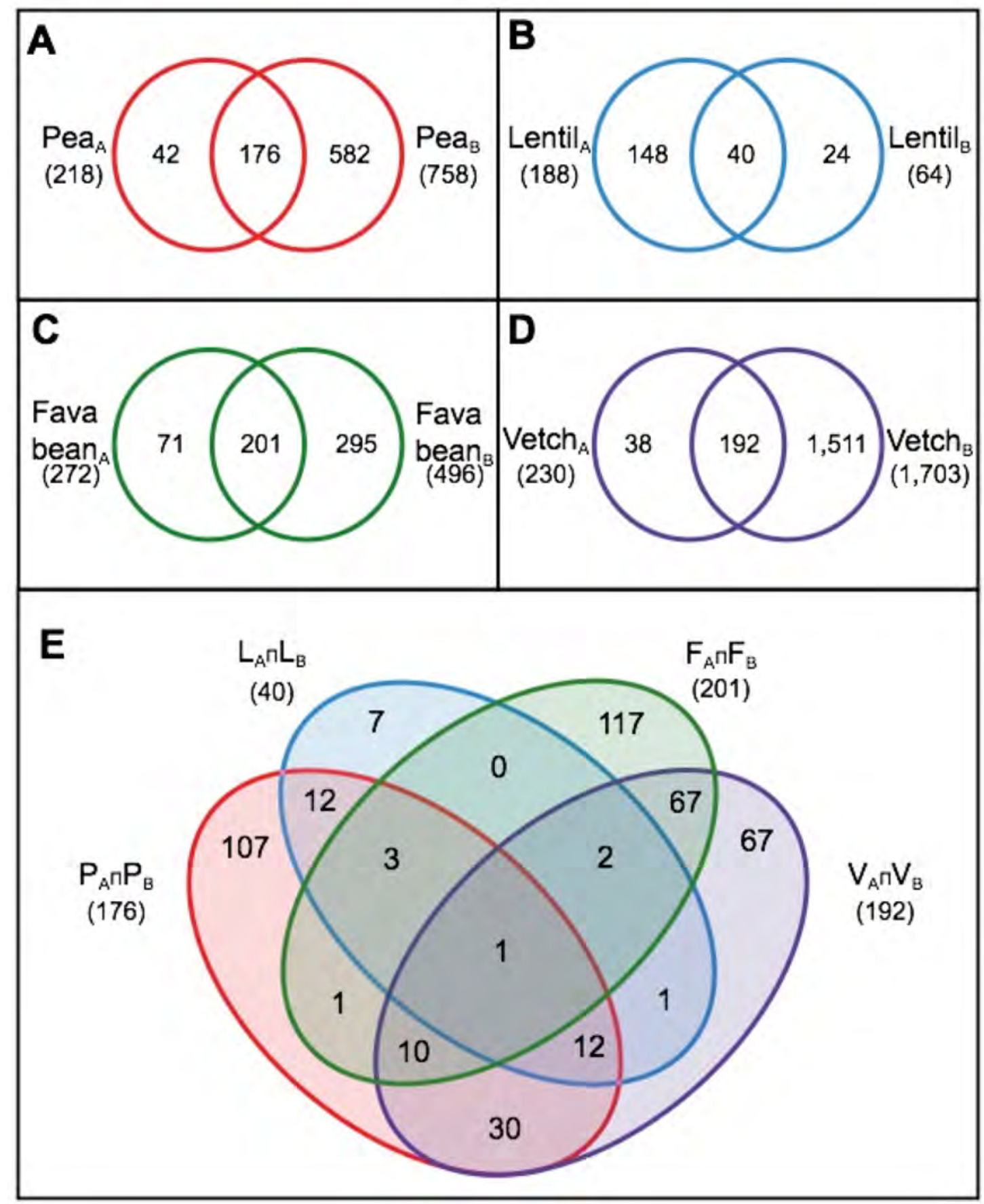

Figure 3.9. Venn diagrams of genes exclusively depleted after five cycles of host selection among mesocosm host-selected subpopulations. Each mesocosm host-selected subpopulation was compared with the initial host-selected subpopulation. $\log _{2}$ values of foldchange were calculated and genes with $\log _{2}$ (fold-change) $<-2$ were considered to be more abundant in mesocosm host-selected Pool-Seqs. The intersections of host-selected subpopulations are plotted in panels $A\left(\right.$ pea $_{A}\left(P_{A}\right)$ and pea $\left.a_{B}\left(P_{B}\right)\right)$, $B\left(\operatorname{lentil}_{A}\left(L_{A}\right)\right.$ and lentil $l_{B}$ $\left(L_{B}\right)$ ), $C$ (fava bean ${ }_{A}\left(F_{A}\right)$ and fava bean $B\left(F_{B}\right)$ ) and $D\left(\operatorname{vetch}_{A}\left(V_{A}\right)\right.$ and $\operatorname{vetch}_{B}\left(V_{B}\right)$ ). The intersection of core absent genes among mesocosm host-selected subpopulations is plotted in panel E. Numbers of genes in each subpopulation and in the different intersections are indicated. 
mesocosm host-selected subpopulation most of the exclusively depleted genes were located in PRL12 (27) and chromosome (24). It is remarkable that genes related with two restriction type I enzyme clusters (pRL11 and chromosome) were depleted, as well as T6SS (imp cluster) and pRL7 tra related genes.

\subsubsection{Genome-wide sequence polymorphisms in Pool-Seq datasets}

A comparative analysis of significant nucleotide polymorphisms in sequences recruited by the reference genome was carried out. The total number of positions that were highly significantly different $\left(F_{\mathrm{ST}} \geq 0.25\right)$ were studied for all pairwise comparisons. From a total of $7,616,370$ positions analysed, only $2,888,679$ were significantly different at least between two subpopulations; Table 3.4 shows the percentage of positions whose differences were highly significant for each pairwise comparison. In order to evaluate the dissimilarities between subpopulations, a global $F_{\mathrm{ST}}$ average was calculated (as the average of all $F_{\mathrm{ST}}$ values) for each pairwise comparison and plotted by multidimensional scaling in a $2 \mathrm{D}$ graphic (Figure 3.10 ). All pairwise comparisons had non-significant average $F_{\mathrm{ST}}$ values $(<0.1$, Panel B). Only small and non-significant, differences were found among (inter) subpopulations, although, as already mentioned in Chapters 1 and 2, the pea initial Pool-Seq was slightly different from the remaining initial Pool-Seqs and from the soil population Pool-Seq. Despite the fact that the observed differences were non-significant, fava bean and lentil subpopulations were more homogeneous than those from pea and vetch (Figure 3.10).

Table 3.4. Percentage of positions that had a highly significant $F_{\mathrm{ST}}$ value $(\geq 0.25)$ for each PoolSeq pairwise comparison.

\begin{tabular}{|c|c|c|c|c|c|c|c|c|c|c|c|c|c|}
\hline & $\mathrm{P}_{\mathrm{A}}$ & $P_{B}$ & $\mathrm{~L}_{\mathrm{A}}$ & $L_{B}$ & $F_{A}$ & $F_{B}$ & $V_{A}$ & $V_{B}$ & $P$ & L & $\mathrm{F}$ & V & $S$ \\
\hline $\mathrm{P}_{\mathrm{A}}$ & 0,00000 & & & & & & & & & & & & \\
\hline$P_{B}$ & 0,70959 & 0,00000 & & & & & & & & & & & \\
\hline$L_{A}$ & 0,34713 & 0,80634 & 0,00000 & & & & & & & & & & \\
\hline$L_{B}$ & 0,38774 & 0,89778 & 0,00035 & 0,00000 & & & & & & & & & \\
\hline$F_{A}$ & 0,79730 & 1,77655 & 0,15182 & 0,02762 & 0,00000 & & & & & & & & \\
\hline$F_{B}$ & 2,18298 & 2,65604 & 1,31316 & 0,77242 & 0,61298 & 0,00000 & & & & & & & \\
\hline$V_{A}$ & 0,32015 & 0,58423 & 0,18037 & 0,11467 & 0,23367 & 1,26344 & 0,00000 & & & & & & \\
\hline$V_{B}$ & 3,76542 & 3,81705 & 3,82191 & 3,78436 & 3,80318 & 4,07654 & 3,76275 & 0,00000 & & & & & \\
\hline $\mathrm{P}$ & 0,41646 & 0,72289 & 0,38646 & 0,34159 & 1,10941 & 2,38532 & 0,41227 & 3,79919 & 0,00000 & & & & \\
\hline $\mathrm{L}$ & 0,41159 & 0,93407 & 0,06628 & 0,02911 & 0,04150 & 0,96656 & 0,09921 & 3,78629 & 0,25361 & 0,00000 & & & \\
\hline$F$ & 0,41706 & 1,24639 & 0,05553 & 0,01887 & 0,03011 & 0,63119 & 0,07852 & 3,86829 & 0,33448 & 0,00004 & 0,00000 & & \\
\hline V & 0,34422 & 0,82422 & 0,06986 & 0,01679 & 0,08114 & 1,25927 & 0,08014 & 3,79613 & 0,17947 & 0,00004 & 0,00000 & 0,00000 & \\
\hline$S$ & 0,95599 & 1,82489 & 0,43084 & 0,17658 & 0,11558 & 0,66865 & 0,30117 & 3,57540 & 1,06014 & 0,04170 & 0,02753 & 0,08912 & 0,00000 \\
\hline
\end{tabular}




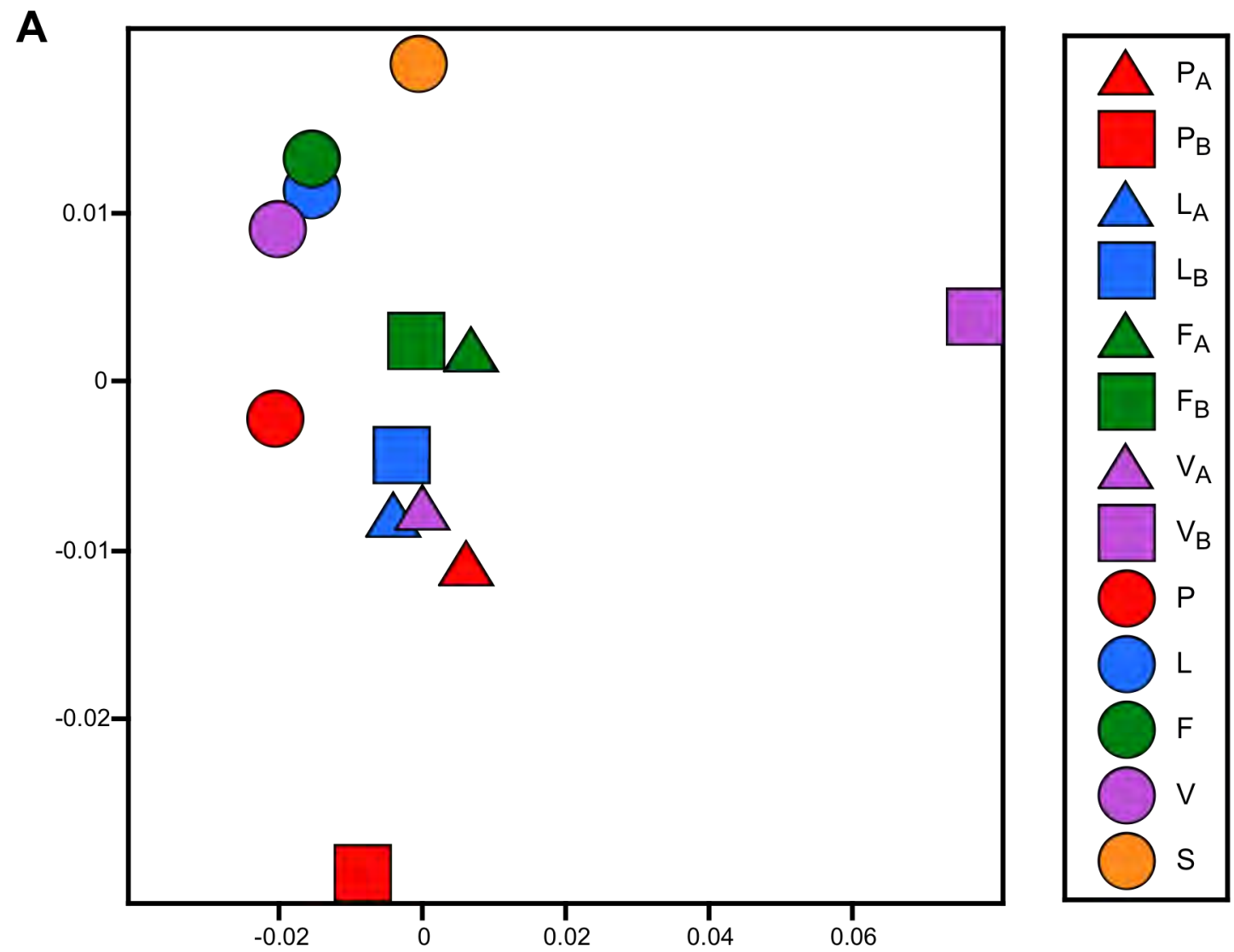

\begin{tabular}{|c|c|c|c|c|c|c|c|c|c|c|c|c|c|}
\hline & $\mathrm{P}_{\mathrm{A}}$ & $P_{B}$ & $\mathrm{~L}_{\mathrm{A}}$ & $L_{B}$ & $\mathrm{~F}_{\mathrm{A}}$ & $F_{B}$ & $V_{A}$ & $V_{B}$ & $P$ & L & $F$ & V & $S$ \\
\hline$P_{A}$ & 0.000 & & & & & & & & & & & & \\
\hline$P_{B}$ & 0.023 & 0.000 & & & & & & & & & & & \\
\hline$L_{A}$ & 0.014 & 0.022 & 0.000 & & & & & & & & & & \\
\hline$L_{B}$ & 0.016 & 0.024 & 0.007 & 0.000 & & & & & & & & & \\
\hline$F_{A}$ & 0.020 & 0.033 & 0.011 & 0.008 & 0.000 & & & & & & & & \\
\hline$F_{B}$ & 0.033 & 0.044 & 0.022 & 0.018 & 0.017 & 0.000 & & & & & & & \\
\hline$V_{A}$ & 0.013 & 0.017 & 0.009 & 0.008 & 0.012 & 0.021 & 0.000 & & & & & & \\
\hline$V_{B}$ & 0.071 & 0.091 & 0.082 & 0.081 & 0.070 & 0.084 & 0.077 & 0.000 & & & & & \\
\hline$P$ & 0.024 & 0.030 & 0.023 & 0.023 & 0.032 & 0.044 & 0.023 & 0.097 & 0.000 & & & & \\
\hline L & 0.030 & 0.041 & 0.023 & 0.021 & 0.023 & 0.036 & 0.023 & 0.093 & 0.014 & 0.000 & & & \\
\hline $\mathrm{F}$ & 0.032 & 0.043 & 0.024 & 0.021 & 0.022 & 0.032 & 0.023 & 0.093 & 0.015 & 0.003 & 0.000 & & \\
\hline V & 0.030 & 0.040 & 0.023 & 0.021 & 0.026 & 0.037 & 0.023 & 0.097 & 0.011 & 0.004 & 0.004 & 0.000 & \\
\hline $\mathrm{S}$ & 0.032 & 0.047 & 0.028 & 0.024 & 0.021 & 0.030 & 0.025 & 0.078 & 0.027 & 0.013 & 0.011 & 0.015 & 0.000 \\
\hline
\end{tabular}

Figure 3.10. Two-dimensional representation of global $F_{\mathrm{ST}}$ average. The multidimensional scaling of global $F_{\mathrm{ST}}$ average is plotted in panel A. Pairwise $F_{\mathrm{ST}}$ comparison matrix among PoolSeqs is plotted in panel $B$. Pool-Seqs are indicated as $P_{A}\left(\right.$ pea $\left._{A}\right), P_{B}($ pea $B), L_{A}\left(\right.$ lentil $\left.A_{A}\right), L_{B}$ (lentil $\left.B_{B}\right), F_{A}\left(\right.$ fava bean $\left.{ }_{A}\right), F_{B}\left(\right.$ fava bean $\left.{ }_{B}\right), V_{A}\left(\right.$ vetch $\left._{A}\right), V_{B}\left(\right.$ vetch $\left._{B}\right), P$ (initial pea), L (initial lentil), $F$ (initial fava bean), V (initial vetch) and $S$ (soil). Plant hosts were colour-coded (see Figure 3.1), and soil was coded orange. Initial host and mesocosm samples A and B are indicated as circles, triangles, and squares, respectively. 


\subsubsection{Global $F_{S T}$ analysis of Pool-Seq coding sequences}

Average $F_{\mathrm{ST}}$ values were obtained for each coding DNA sequence (CDS) of the Rhizobium leguminosarum bv. viciae 3841 reference genome for each pairwise comparison among Pool-Seqs. A total of 7,269 CDS were analysed and both highly significant $(\geq 0.25)$ and non-significant $(<0.1)$ CDS $F_{S T}$ values among host subpopulations (subpopulation $_{A}$, subpopulation s $_{B}$ and initial subpopulation) and between mesocosm Pool-Seqs (subpopulation ${ }_{A}$, subpopulaton ${ }_{B}$ ) were investigated.

Out of the total CDSs studied, 1,243 were non-informative: they had $F_{\mathrm{ST}}=0$ in all subpopulation comparisons. In most cases, the nil value of $F_{\mathrm{ST}}$ was obtained because no reads were recruited for that CDS in at least one of the Pool-Seqs, as a direct result of the comparison algorithm. Only $11 \mathrm{CDSs}$ were conserved $(\mathrm{BPKM}>10)$ in all PoolSeqs analysed and showed $F_{\mathrm{ST}}=0$ because of their complete conservation (Table 3.5). A further $88 \mathrm{CDSs}$ were non-significantly different in all comparisons $\left(F_{\mathrm{ST}}<0.1\right)$ among Pool-Seqs (Supplementary Table 3.S1). It is worth noting that the three copies of $5 \mathrm{~S}$ rRNA, as well as a high number of tRNAs (36 out of 52), were conserved among PoolSeqs. Surprisingly, the three copies of $16 S$ and $23 S$ rRNAs were not preserved in all

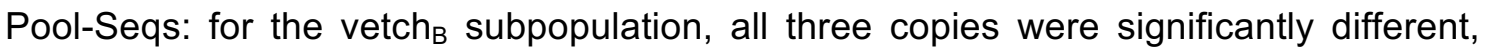
while for the fava bean ${ }_{B}$ subpopulation only copy 1 was significantly different (Figure 3.11). These 1,331 CDSs were thus omitted from the analysis and the remaining 5,398 CDSs were investigated. These results are presented in Supplementary Tables S6-S9.

Table 3.5. Non-informative CDSs present in all Pool-Seqs

\begin{tabular}{llll}
\hline Product & Gene & & \\
\hline Cytochrome C protein & RL1160 & & \\
Putative CBS domain protein & pRL100290 & & \\
$\begin{array}{l}\text { Putative plasmid stabilisation (kluAB) } \\
\text { transposase for insertion sequence }\end{array}$ & pRL120267 & pRL120268 & \\
element & RL1973 & & \\
Conserved hypothetical protein & & & \\
Hypothetical protein & pRL90280 & pRL90291 & \\
Pseudogene & RL0552 & RL0888 & pRL110057 \\
\hline
\end{tabular}




\subsection{Host-selected CDSs (intra-host comparisons)}

We consider that a CDS is plant-host selected when it shows a non-significant $F_{\mathrm{ST}}$ value $\left(F_{S T}<0.1\right)$ for all host-selected subpopulation (subpopulation $n_{A}$, subpopulaton ${ }_{B}$ and initial subpopulation) comparisons. Different numbers of CDSs met this criterion: 1,143 CDS for pea subpopulations, 5,049 CDS for lentil subpopulations, 976 for fava bean subpopulations and only 72 CDS for vetch subpopulations (Supplementary Table S5).

Among the 1,143 CDSs that showed a non-significant $F_{\mathrm{ST}}$ between each pea pairwise comparison ( pea $_{A}$, pea $a_{B}$ and initial pea), the absence of symbiosis-related genes was noteworthy. We can highlight the selection of genes related with chemotaxis (cheYD) and motility (fliNF, flgBC, fliE, flaF, flbT and fliR), ATP synthase I (atp/CF), numerous ribosomal proteins and $A B C$ transporters, modification of fatty acids (fabF1F2Z, acpXL) and EPS synthesis (pssV, pssONT and pssBA), as well as $16 S$ and $23 S$ rRNAs. On the contrary, many of the CDSs selected in fava bean subpopulations, were symbiosis-related genes ( $f d x B$, nifN, nifK, nifH, rhil, rhiABCRD,
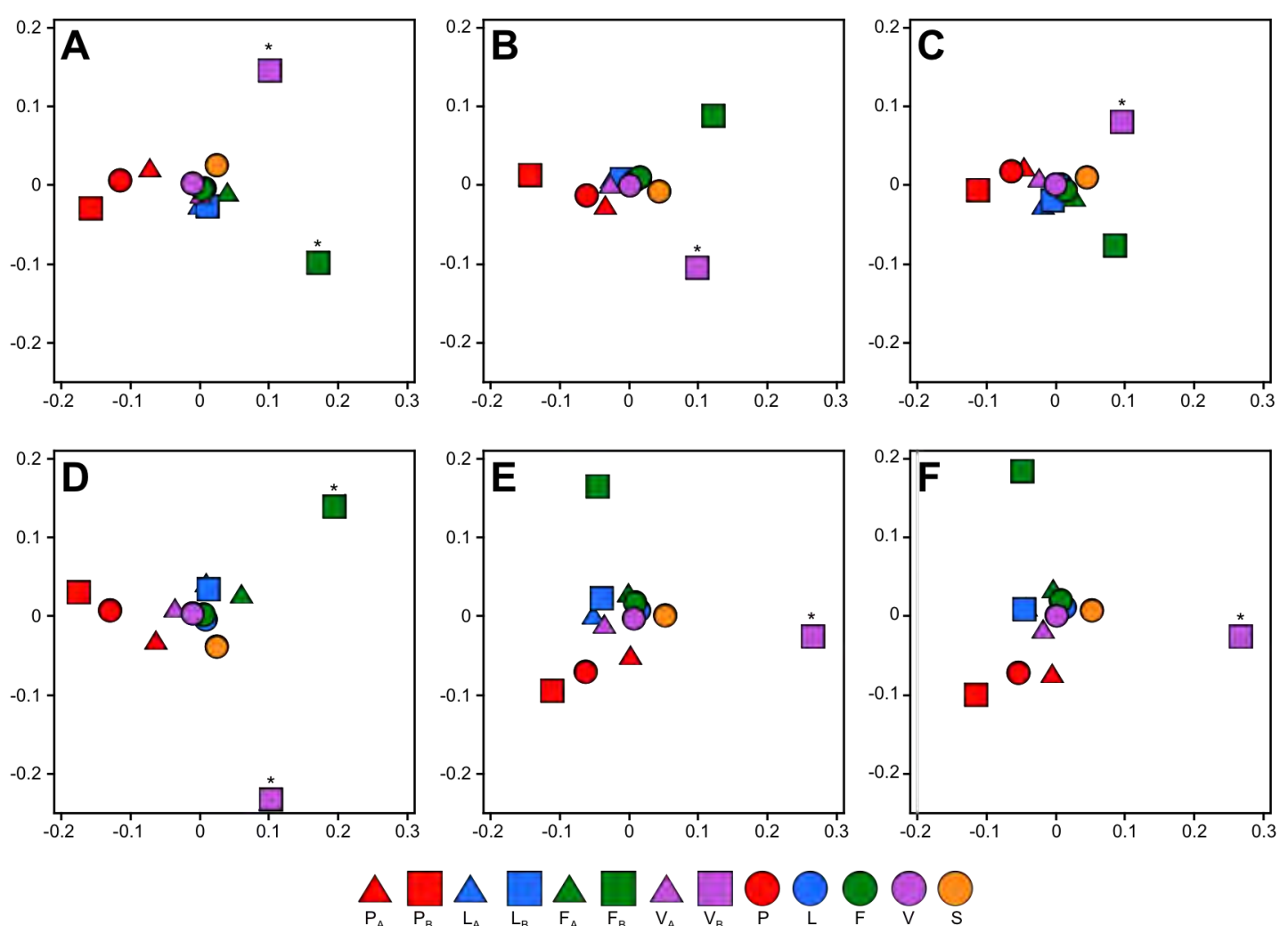

Figure 3.11. Two-dimensional representation of $F_{\mathrm{ST}}$ for $16 \mathrm{~S}$ and $23 \mathrm{~S}$ rRNA genes. Multidimensional scaling (MDS) is based on pairwise $F_{S T}$ average values of: $A$ (16S_1), B (16S_2), C (16S_3), D (23S_1), E (23S_2) and $F$ (23S_3). Pool-Seqs are indicated as $P_{A}$ $\left(\right.$ pea $\left._{A}\right), P_{B}\left(\right.$ pea $\left._{B}\right), L_{A}\left(\right.$ lentil $\left._{A}\right), L_{B}($ lentil $B), F_{A}\left(\right.$ fava bean $\left.A_{A}\right), F_{B}($ fava bean $B), V_{A}\left(\right.$ vetch $\left._{A}\right), V_{B}$ (vetch ${ }_{B}$ ), $P$ (initial pea), $L$ (initial lentil), $F$ (initial fava bean), V (initial vetch) and $S$ (soil). Plant hosts were colour-coded (see Figure 3.1), and soil was coded orange. Initial host and mesocosm samples $A$ and $B$ are indicated as circles, triangles, and squares, respectively. indicates highly significant $F_{\mathrm{ST}}$ values (over 0.25 ). 
nodN, nodLEFDABCl, nifAfixXC and fixNOQPGfnrN from pRL10, and fixSI and fixQNK from $\mathrm{pRL9}$ ), in addition to numerous ribosomal proteins and $A B C$ transporters and copies 2 and 3 of $16 S$ and $23 S$ rRNAs. Lentil subpopulations showed the highest number of selected CDSs, a number so high $(5,049)$ that suggests that lentils did not select at all. Finally, vetch subpopulations selected just a few CDSs (72), most of them related with transcriptional regulation. Among them was an fnr $N$-like transcriptional activator (pRL100211).

\subsection{Non-host selected CDSs (intra-host comparisons)}

Some CDSs showed a highly significant $F_{\mathrm{ST}}$ value $\left(F_{\mathrm{ST}} \geq 0.25\right)$ in intra-host subpopulation comparisons (pairwise comparisons of subpopulation ${ }_{A}$, subpopulaton su $_{B}$ and initial subpopulation). We denominate these as "non-host selected" CDSs and reflect genotype heterogeneities among subpopulations selected by the same host. Different numbers of CDSs met this criterion: 0 CDSs for lentil and fava bean subpopulations, 17 CDSs for vetch subpopulations and, notably, 474 CDSs for pea subpopulations (Supplementary Table S6). The pea result was unexpected because pea $_{\mathrm{A}}$ and pea $_{\mathrm{B}}$ had been designed as replicates. Among the 474 pea "non-host selected" CDSs were genes annotated as fixG (pRL100208), as related to cobalamine synthesis (cobUW, RL2831, RL2832), or related to siderophore synthesis (iroE, vbsPS). Most of the 17 vetch CDS were annotated as transcriptional regulators.

\subsection{Mesocosm host-selected CDSs (intra-host comparisons)}

Those genes that had a non-significant $F_{\mathrm{ST}}$ value $\left(F_{\mathrm{ST}}<0.1\right)$ in the intra-host pairwise comparison of subpopulation $\mathrm{A}_{\mathrm{A}}$ and subpopulation $_{B}$ were considered to be "selected CDSs" in the mesocosm experiment. The rationale for this analysis is that some genes could be host-selected only after repeated cycles of plant growth, and not in the initially selected subpopulation. Obviously, all those genes that were selected in intra-host comparisons (see section 6.2.1.7.1, above) were also selected in mesocosm intra-host comparisons. Numbers of CDSs exclusively selected within each hostselected mesocosm subpopulations varied: 643 for pea, 239 for lentil, 1,321 for fava bean and 87 for vetch (Supplementary Table S7). Among the 643 genes selected by pea mesocosm subpopulations were some symbiosis-related genes (nifEK, nifH, nodTMN, nodF, nodABCIJ, nifBAfixXCBA and fixOQ from pRL10 and fixSIHGPQ and fixK from pRL9), chemotaxis (cheXY, cheRB) and motility (fliF, fliG, fliM, flil, flaC, flgE and $f(g D)$ related genes, glutamine synthetase $(g \ln A)$, different ribosomal proteins, and fnrN (RL2818), as well as different ABC transporters. Among the 239 CDSs selected 
by the lentil mesocosm were genes annotated as chemotaxis-related proteins (cheWA, $m c p C$ ) or ribosomal proteins. Within fava bean, among the 1,321 genes selected during the mesocosm experiment, were most of the remaining symbiotic-related genes (nifE, nifD, nodO, nodTM, nodJ, nifB, fixBA from $\mathrm{pRL10}$, and fixPO from $\mathrm{pRL}$ ), LPS biosynthesis-related genes (fabD, fabG, pssU, pssV, pssM, pssPONT), fnrN (RL2818), lacZ (RL4248), rpoE (RL4524), rosR (RL1379) and copy 1 of $16 S$ and $233 S$ rRNAs. Regarding vetch, the 87 CDSs selected included some $A B C$ transporters, but no symbiosis-related genes.

\subsection{Mesocosm non-host selected CDSs (intra-host comparisons)}

Likewise, we considered those genes that had a highly significant $F_{\mathrm{ST}}$ value $\left(F_{\mathrm{ST}} \geq 0.25\right)$ in the intra-host pairwise comparison of host $\mathrm{A}_{\mathrm{A}}$ and host $_{\mathrm{B}}$ subpopulations to be "non-host selected CDSs" in the mesocosm experiment. As previously, all genes that were non-host selected in intra-host comparisons (section 6.2.1.7.2) were also non-host selected in mesocosm experiments. Different numbers of CDS were exclusively non-host selected within each host-selected mesocosm subpopulation: 2,005 for pea, 65 for lentil, 139 for fava bean and 5,461 for vetch (Supplementary Table S8). It is noteworthy that, among the 2,005 CDS non-selected by pea mesocosms, there were motility-related genes (pilQA, cpaDE, tadC, fliL, flaA, motB, motD and fliQ), rosR (RL1379), rpoBC (RL1766, RL1767), gInll (RL3549) and siderophore-related genes ( $v s b O, v s b A D L$ and $v s b C)$. In lentil, only 65 CDS were not selected in the mesocosm, such as attS (RL2331) or TRAP transporter (RL4474RL4476). Among the 139 genes non-selected by the fava bean mesocosm experiment, there was a tRNA-Trp (RLt58). A high number of genes $(5,461)$ were non-host selected in the vetch mesocosm experiment, which reflected the large dissimilarity of vetch $\mathrm{A}_{\mathrm{A}}$ and vetch $_{B}$ subpopulations. This dissimilarity was due to the selection of a different

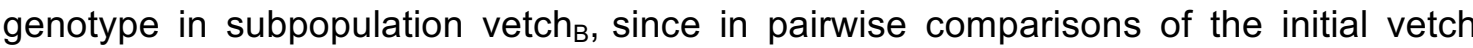
population with vetch ${ }_{\mathrm{A}}$ and vetch $\mathrm{B}_{\mathrm{B}}$ subpopulations 58 and $5,471 \mathrm{CDSs}$ had $F_{\mathrm{ST}} \geq 0.25$, respectively.

\subsubsection{8. $F_{S T}$ analysis of specific coding sequences in Pool-Seq datasets}

In an attempt to understand the forces that govern plant-host genotype selection, we focused our polymorphism analysis on specific, relevant genes and cluster genes. For each of the chosen regions, an average $F_{\mathrm{ST}}$ value was obtained for each pairwise comparison among subpopulations, a matrix of $F_{\mathrm{ST}}$ values was constructed, and distances derived from it were plotted by multidimensional scaling in 2D graphics, 
presented below. In all cases, the distances separating points reflect the overall differences in $F_{\mathrm{ST}}$ values among subpopulations. Chosen regions have been arbitrarily divided into "housekeeping," "symbiotic," "rhizospheric," and "differential among hosts" categories, and are analysed below.

\subsection{Housekeeping marker regions}

Seven different housekeeping regions were analyzed (Figure 3.12). Seven of them were: the three copies of rRNA (16S rRNA-tRNA-ile-tRNA-ala-23S rRNA-5S rRNA), $a t p D, g I n l l, r e c A$ and $r p o B$. Results for these marker regions were compared with a
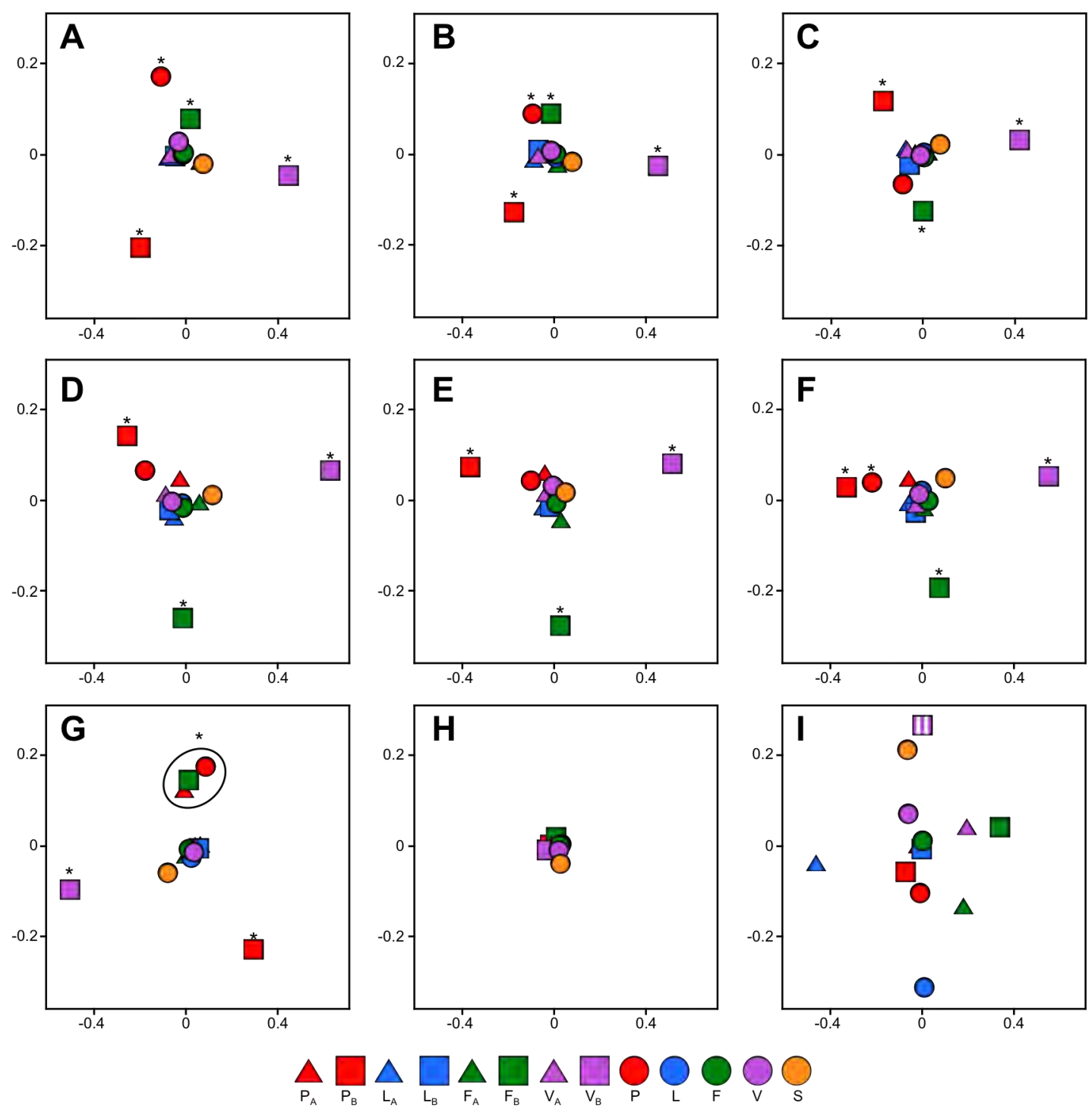

Figure 3.12. Two-dimensional representation of $F_{S T}$ for some housekeeping markers. Multidimensional scaling (MDS) is based on pairwise $F_{S T}$ average values of: $A$ (rDNA_1), B (rDNA_2), C (rDNA_3), D (atpD), E (glnll), F (recA), G (rpoB), H (5S-1) and I (hyfE). Pool-Seqs are indicated as $P_{A}\left(\right.$ pea $\left._{A}\right), P_{B}\left(\right.$ pea $\left._{B}\right), L_{A}\left(\right.$ lentil $\left.A_{A}\right), L_{B}($ lentil $), F_{A}\left(\right.$ fava bean $\left.{ }_{A}\right), F_{B}\left(\right.$ fava bean bea $\left._{B}\right), V_{A}$ $\left(\right.$ vetch $\left._{A}\right), V_{B}\left(\right.$ vetch $\left._{B}\right), P$ (initial pea), L (initial lentil), $F$ (initial fava bean), V (initial vetch) and $S$ (soil). Plant hosts were colour-coded (see Figure 3.1), and soil was coded orange. Initial host and mesocosm samples $A$ and $B$ are indicated as circles, triangles, and squares, respectively. * indicates highly significant $F_{\mathrm{ST}}$ values (over 0.25). Circles group subpopulations with no significant $F_{\mathrm{ST}}$ among them. White bars within a subpopulation symbol indicate a low coverage value (below 10 BPKM) for at least one gene in the region analysed. 
marker gene that showed no selection by any subpopulation (5S; RLr03; Figure 3.15 H) and with a marker that provided a clear differentiation of plant-associated genotypes for most subpopulations (hyfE, pRL110295; Figure 3.15 I). For all these markers, subpopulations pea $_{B}$ (red square), fava bean $_{B}$ (green square) and vetch (purple square) consistently selected for significantly different genotypes. Other significantly different genotypes were selected for in rRNA1, rRNA2, recA and rpoB by the pea initial population, and for $r p o B$ by the pea $a_{A}$ mesocosm subpopulation. The remaining subpopulations clustered together for all seven markers. Finally, only for the lentil host did all three subpopulations group together.

\subsection{Symbiosis-related marker regions}

Nine different symbiosis-related regions were analyzed (Figure 3.13). A similar pattern was found for six of these regions: nif (fdxBnifNEKDH, pRL100156pRL100162), rhi (rhil----rhiABCRD, pRL100164-pRL100173), nod (nodO-nodTNMLEFDABCIJ, pRL100175-pRL100189), nif-fix (nifBAfixXCBA, pRL100195pRL1001200), fix (fixNOQPGHISfnrN-like, pRL100205-pRL100211) clusters and the symbiotic region (56 genes, $f d x B$ through fnrN-like, pRL100156-pRL100211), all of them located in $\mathrm{pRL} 10$. Subpopulations pea $_{A}$, pea $_{B}$ and vetch $_{B}$ selected genotypes that were different, with high significance, from those found in the remaining subpopulations. Regarding host-specific subpopulations, none of the three fava bean subpopulation genotypes differed significantly, although fava bean $n_{B}$ was divergent with respect to other host populations, while fava bean $\mathrm{f}_{\mathrm{A}}$ and initial fava bean were not. Likewise, the initial pea subpopulation selected the same genotype as lentil and vetch for all symbiotic clusters and symbiotic region; whereas pea and fava bean shared the same initial genotype only for nod and fix clusters, while initial pea and soil shared genotpyes for nif and nod clusters and for the symbiotic region. The whole symbiotic plasmid pRL10 was also chosen as a region of interest (Figure 3.13 panel G). It is noteworthy that fava bean subpopulations, rather homogeneous for six symbiotic regions within pRL10 (see above), did not select the same symbiotic plasmid genotype. This was also the case for all pea subpopulations. Regarding the two chromosomal "symbiotic" markers (fnrN (RL2818) and rosR (RL1379); panels H and I respectively), different patterns were found. For $f n r N$, only vetch $_{B}$ selected genotype that was significantly different, not only from other vetch subpopulations, but also from other hosts. These remaining subpopulations showed a complex genotype selection pattern, where initial lentil, fava bean, vetch, soil, lentil ${ }_{A}$, lentil ${ }_{B}$ and fava $a_{A}$ shared the same fnrN genotype. Pea mesocosm subpopulations showed the same genotype as lentil 
subpopulations, vetch ${ }_{A}$ and initial vetch; likewise pea $_{A}$ clustered with fava bean ${ }_{A}$ but this was not the case for pea $_{\mathrm{B}}$. rosR and the symbiotic plasmid showed a similar pattern of variation as that found for housekeeping markers.
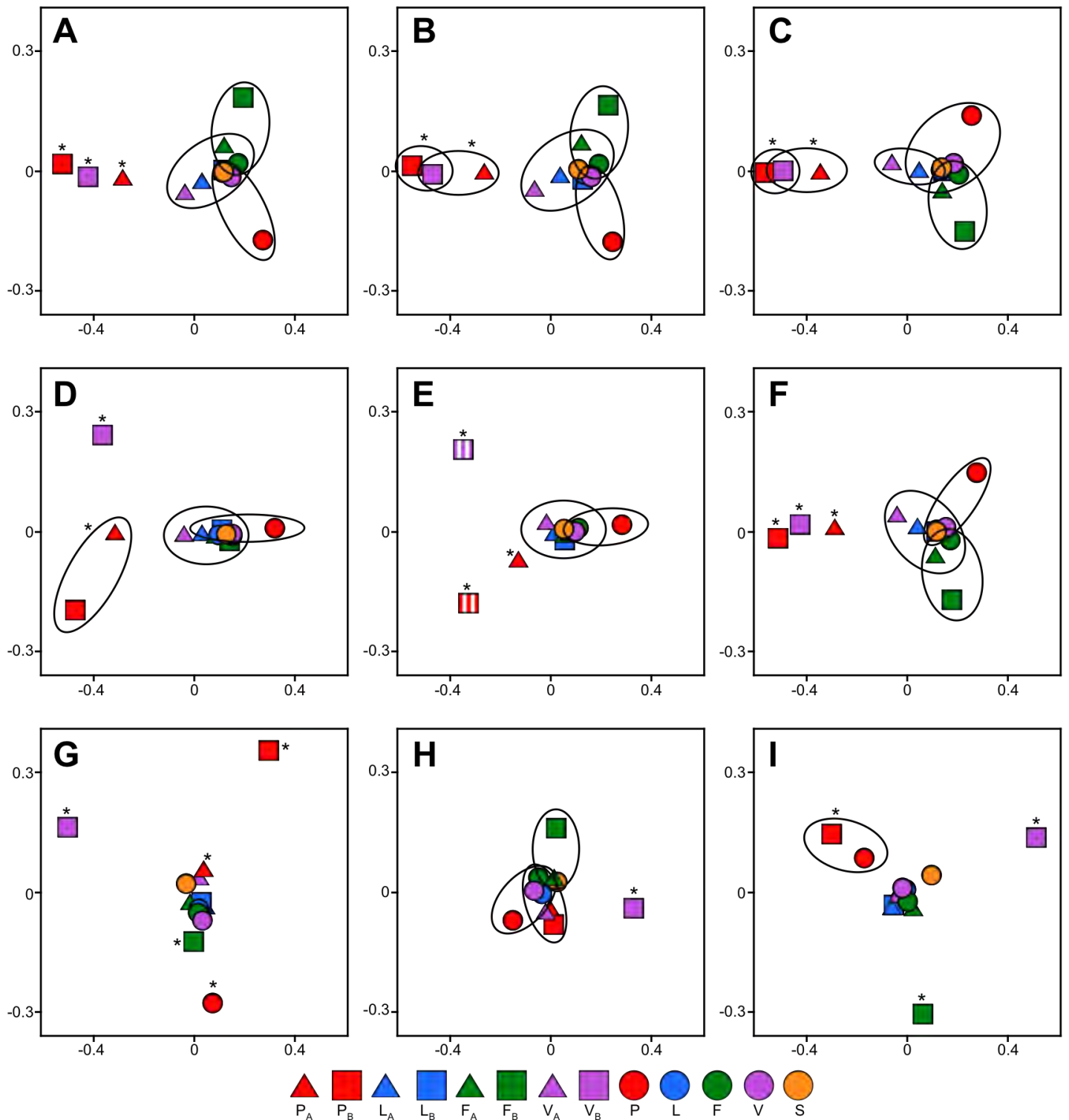

Figure 3.13. Two-dimensional representation of $F_{S T}$ for some symbiosis-related markers. Multidimensional scaling (MDS) is based on pairwise $F_{\mathrm{ST}}$ average values: A (nif), B (rhi), C (nod), D (nif-fix), E (fix), F (symbiotic region), G (symbiotic plasmid), H (fnrN) and I (rosR). Pool-Seqs are indicated as $P_{A}\left(\right.$ pea $\left._{A}\right), P_{B}\left(\right.$ pea $\left._{B}\right), L_{A}$ (lentil $\left.\left.\right|_{A}\right), L_{B}\left(\right.$ lentil $\left.\left.\right|_{B}\right), F_{A}$ (fava bean $\left.{ }_{A}\right), F_{B}$ (fava bean $B$ ), $V_{A}\left(\right.$ vetch $\left._{A}\right), V_{B}\left(\right.$ vetch $\left._{B}\right), P$ (initial pea), $L$ (initial lentil), $F$ (initial fava bean), $V$ (initial vetch) and $S$ (soil). Plant hosts were colour-coded (see Figure 3.1), and soil was coded orange. Initial host and mesocosm samples A and B are indicated as circles, triangles, and squares, respectively. ${ }^{*}$ indicates highly significant $F_{\mathrm{ST}}$ values (over 0.25 ). Circles group subpopulations with no significant $F_{\mathrm{ST}}$ among them. White bars within a subpopulation symbol indicate a low coverage value (below 10 BPKM) for at least one gene in the region analysed. 


\subsection{Rhizospheric marker regions}

Four different regions, potentially implicated in rhizosphere fitness, were studied: che (mcp cheXYAWRBYD-, RL08685-RL0894), fab (IpxXL adhA2 fabF1F2Z acpXL, RL2812-RL2817), pss (pssSRVLKJIHGFMDE, RL3642-RL3655) and prs (prsD/E prsED plyA, RL3656-RL3659) clusters (Figure 3.14). In all regions pea ${ }_{\mathrm{B}}$, fava bean $\mathrm{B}_{\mathrm{B}}$ and vetch $_{B}$ showed were significantly different, when compared to the remaining subpopulations. Regarding hosts, the pea subpopulations were consistently different to other host populations only for che and pss clusters. These results are comparable to those observed in housekeeping regions (see 6.2.1.8.1).
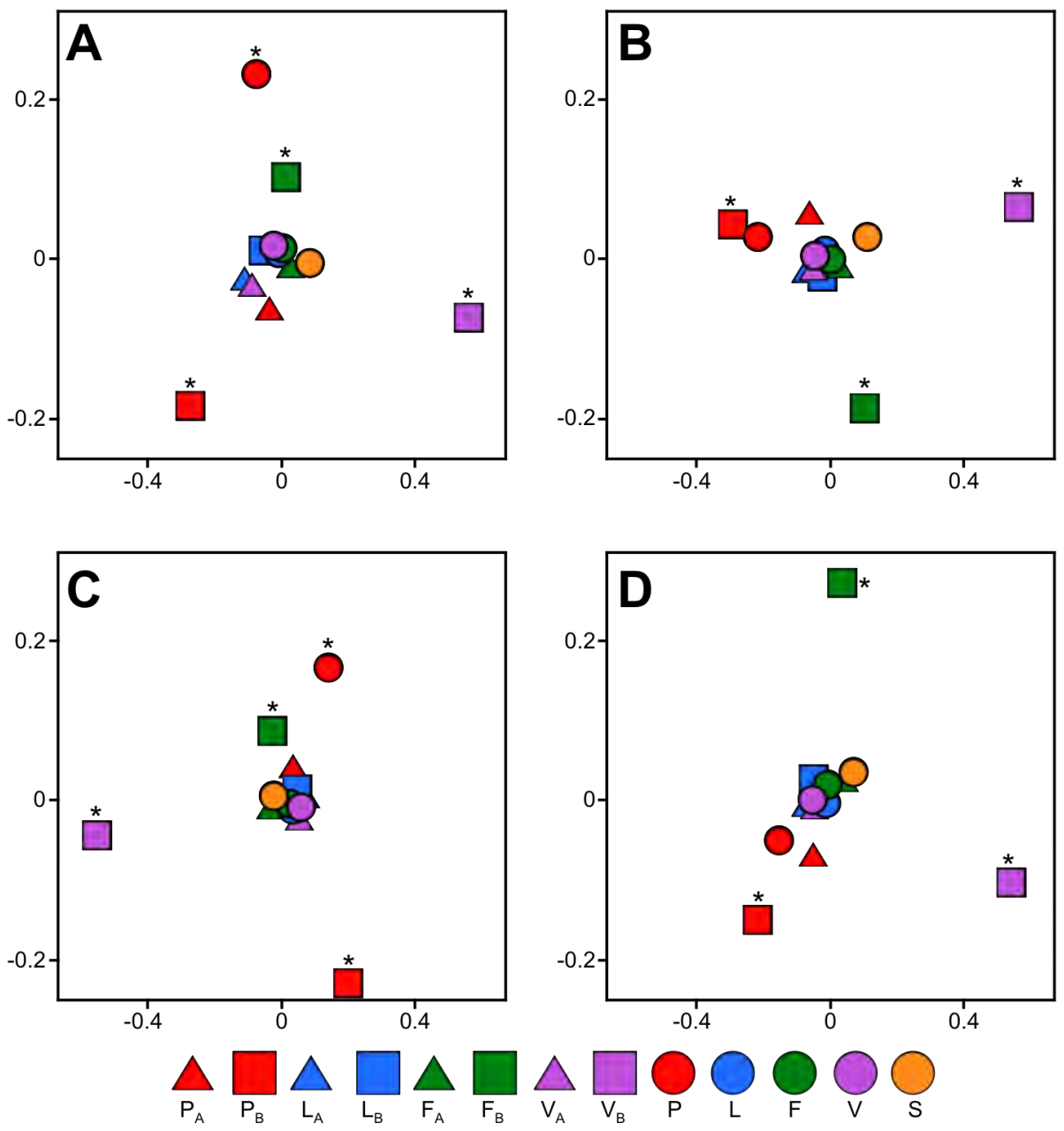

Figure 3.14. Two-dimensional representation of $F_{\mathrm{ST}}$ for some rhizospheric markers. Multidimensional scaling (MDS) is based on pairwise $F_{\mathrm{ST}}$ average values: $\mathrm{A}(\mathrm{che}), \mathrm{B}(\mathrm{fab}), \mathrm{C}(\mathrm{exo})$ and $\mathrm{D}(p r s)$. Pool-Seqs are indicated as $\mathrm{P}_{\mathrm{A}}$ $\left(\right.$ pea $\left._{A}\right), P_{B}\left(\right.$ pea $\left._{B}\right), L_{A}\left(\right.$ lentil $\left.I_{A}\right), L_{B}\left(\right.$ lentil $\left.{ }_{B}\right), F_{A}\left(\right.$ fava bean $\left.{ }_{A}\right), F_{B}\left(\right.$ fava bean $\left.{ }_{B}\right), V_{A}$ $\left(\right.$ vetch $\left._{A}\right), V_{B}\left(\right.$ vetch $\left._{B}\right), P$ (initial pea), $L$ (initial lentil), $F$ (initial fava bean), $V$ (initial vetch) and $S$ (soil). Plant hosts were colour-coded (see Figure 3.1), and soil was coded orange. Initial host and mesocosm samples A and B are indicated as circles, triangles, and squares, respectively. ${ }^{*}$ indicates highly significant $F_{\mathrm{ST}}$ values (over 0.25). 
6.2.1.8.4. Differential regions among host-selected subpopulations (inter-host comparisons)

$F_{\mathrm{ST}}$ analysis results were scanned in search of differential regions among hostselected Pool-Seqs, in order to find genes or gene clusters presenting host-selected genotypes. Four gene clusters were selected, notably in the symbiotic (three) and pRL12 (one) plasmids: qat6 (qat6VWX, pRL100079-pRL100081, quaternary ammonium transporter of $A B C$ family), pRL100127-pRL100131 (HAAT ABC transporter), att (attJKLM, pRL100133-pRL100136, $\mathrm{\gamma}$-hydroxybutyrate degradation cluster) and dct (dctMQ-, pRL120498-pRL120500, protocatechuate transporter) (Figure 3.15). For qat6, the vetch $_{B}$ subpopulation showed a significantly different genotype when compared to the remaining subpopulations. The same was true for $p e a_{A}, p a_{B}$ and vetch $_{\mathrm{A}}$, that clustered together and were significantly different from the rest of PoolSeqs. All three fava bean subpopulations selected the same genotype, and the same happened with initial pea, initial lentil, initial fava bean, initial vetch, soil and mesocosm lentil $_{B}$. Lentil $A_{A}$ showed similarity to that Pool-Seq group, with the exception of initial vetch (Figure 3.15, panel A). The HAAT ABC transporter localized in pRL100127pRL100131 was plotted in panel $B$, and defined different groups On one side, pea $a_{A}$, pea $_{B}$ and vetch ${ }_{B}$ clustered together, whereas soil and vetch ${ }_{A}$ were localized alone; all of them were clearly different from the remaining subpopulations. These clustered in two different groups: lentilA and lentil, on one hand, and initial host-selected Pool-Seqs plus fava bean mesocosm subpopulations, on the other. A similar pattern was detected for att (panel C), except that fava bean mesocosm Pool-seqs clustered independently of initial host-selected subpopulations. The above three clusters are localized at the initial part of the symbiotic plasmid ( $p R L 10)$, a region that we previously defined as lowcoverage in most subpopulations (see 6.2.1.3). However, all these genes had BPKM values above our cut-off of 10 for all subpopulations. Cluster dct, localized in plasmid pRL12, had been classified as "non host-selected" in fava bean subpopulations, because two significantly different genotypes were selected in the two mesocosm experiment replicates. Complex relationships were detected for the rest of Pool-Seqs, and two groups can be defined: on one-side, pea subpopulations and vetch $\mathrm{B}_{\mathrm{B}}$, and, on the other side, initial lentil, initial fava bean, initial vetch, lentil ${ }_{B}$ and soil. Lentil ${ }_{A}$ and vetch $_{\mathrm{A}}$ bridged both groups. 

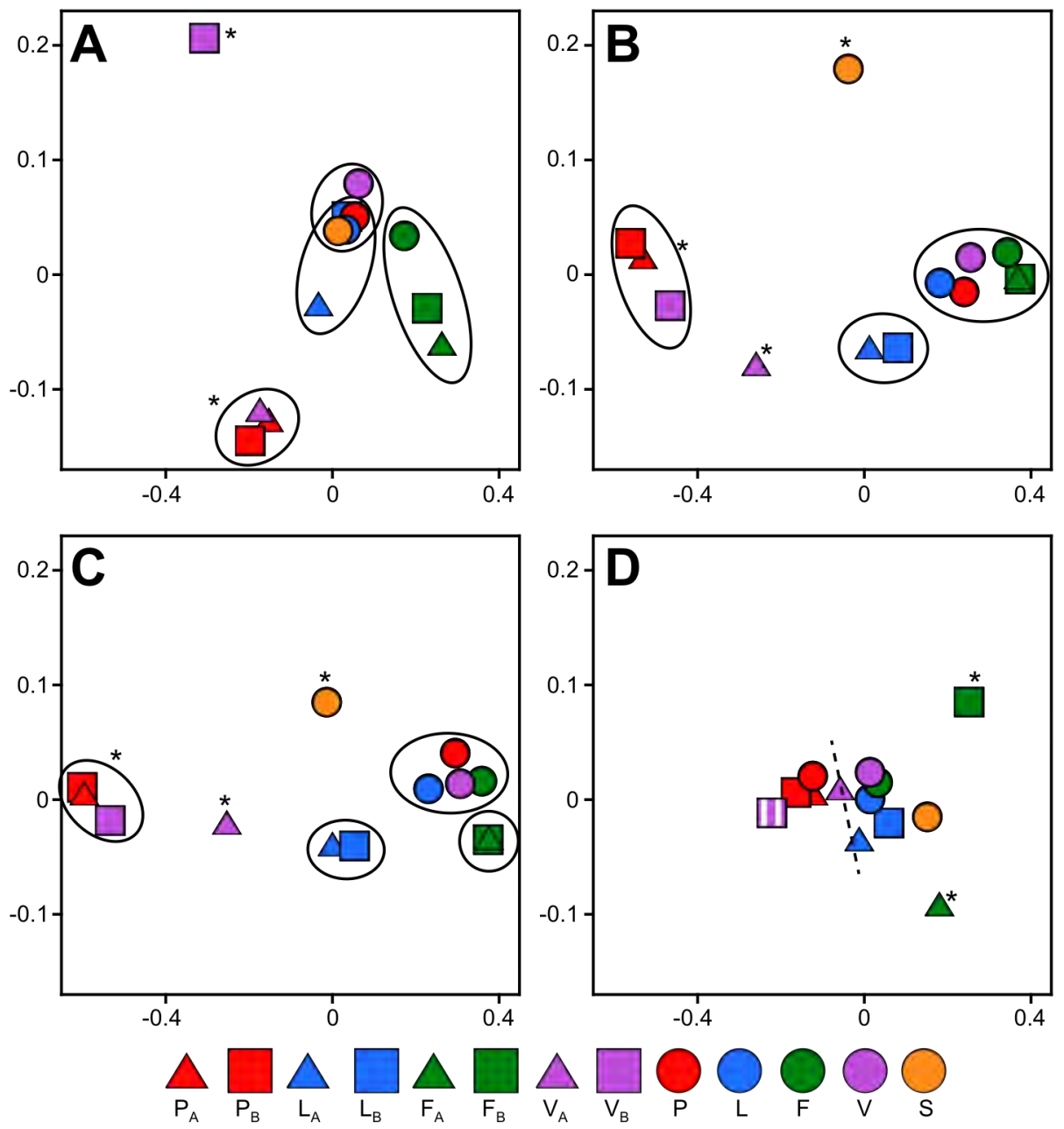

Figure 3.15. Two-dimensional representation of $F_{S T}$ for differential regions (inter-host). Multidimensional scaling (MDS) is based on pairwise $F_{\mathrm{ST}}$ average values: $\mathrm{A}$ (qat6), B (HAAT ABC transporter), $\mathrm{C}$ (att) and $\mathrm{D}$ (dct). Pool-Seqs are indicated as $P_{A}\left(\right.$ pea $\left._{A}\right), P_{B}\left(\right.$ pea $\left._{B}\right), L_{A}\left(\right.$ lentil $\left.\left.\right|_{A}\right), L_{B}\left(\right.$ lentil $\left.B_{B}\right), F_{A}$ (fava bean $\left.{ }_{A}\right), F_{B}$ (fava bean $\left.B_{B}\right), V_{A}\left(\right.$ vetch $\left._{A}\right), V_{B}$ (vetch $\left.{ }_{B}\right), P$ (initial pea), $L$ (initial lentil), $F$ (initial fava bean), $V$ (initial vetch) and $S$ (soil). Plant hosts were colour-coded (see Figure 3.1), and soil was coded orange. Initial host and mesocosm samples A and $\mathrm{B}$ are indicated as circles, triangles, and squares, respectively. ${ }^{*}$ indicates highly significant $F_{\mathrm{ST}}$ values (over 0.25 ). Circles group subpopulations with no significant $F_{\mathrm{ST}}$ among them, and a discontinuous line separates groups with $F_{\mathrm{ST}}$ values between $0.1-0.25$. White bars within a subpopulation symbol indicate a low coverage value (below 10 BPKM) for at least one gene in the region analysed. 
Six individual genes were also selected: RL1858 (protease), RL2862 (hypothetical protein), pRL110330 (MerR type transcriptional factor), pRL100104 (phaE), pRL100107 (hypothetical protein) and pRL100120 (hypothetical protein) (Figure 3.16).

RL1858 and pRL110330: Three genotypes were defined, that of pea subpopulations plus vetch ${ }_{B}$; that of soil and fava bean $n_{B}$; and that of and lentil subpopulations plus initial fava bean, fava bean $_{\mathrm{A}}$, initial vetch and vetch . $_{\text {. }}$ (Figure 3.16 A)

RL2862: Pea subpopulations clustered together, while fava bean ${ }_{B}$ and vetch $_{B}$ showed significantly different genotypes. The remaining subpopulations grouped jointly. The three above genes appeared to be absent (BPKM below 10) in vetch . (Figure $3.16 \mathrm{~B}$ )
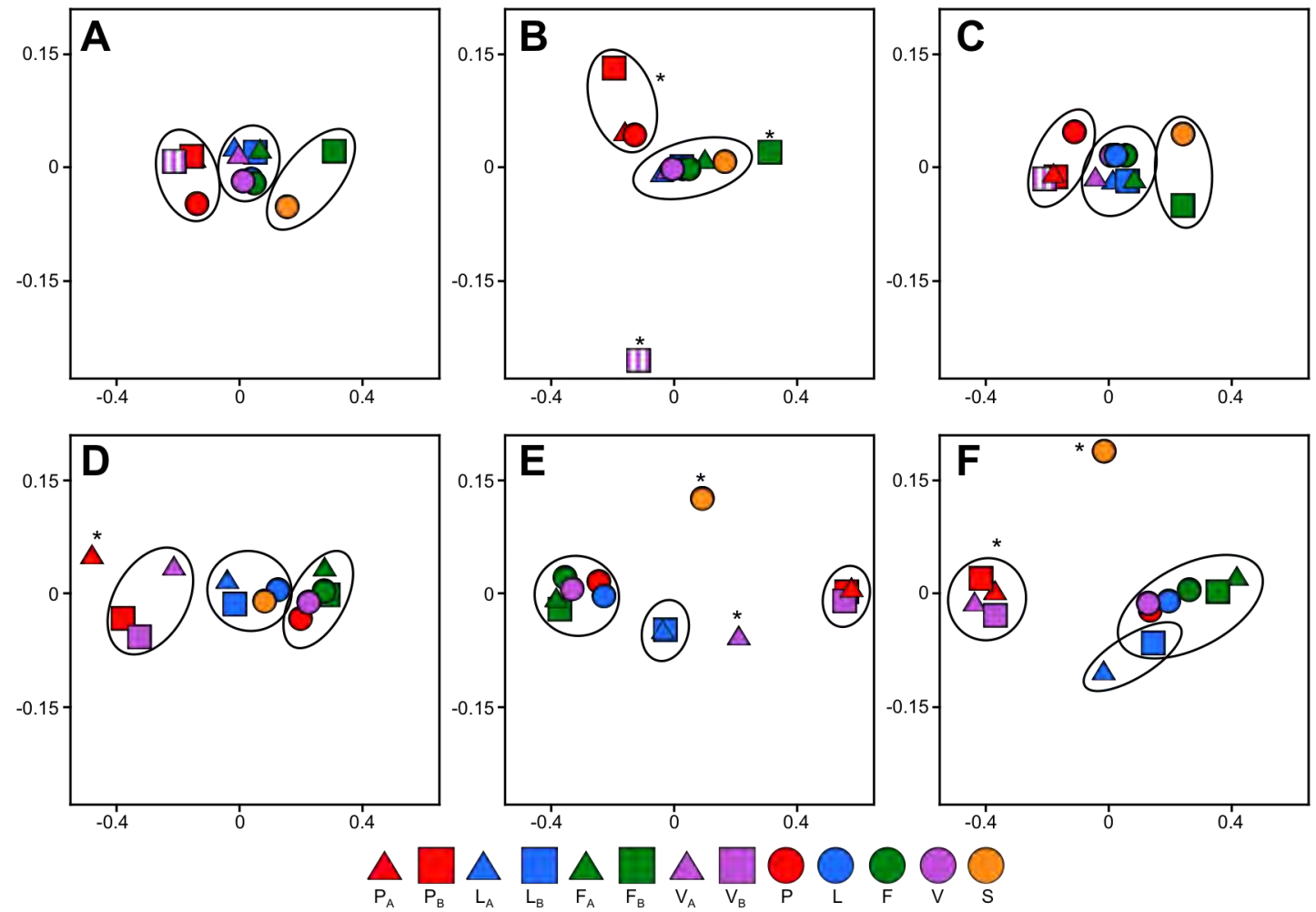

Figure 3.16. Two-dimensional representation of $F_{\mathrm{ST}}$ for differential genes (inter-host). Multidimensional scaling (MDS) is based on pairwise $F_{S T}$ average values: A (RL1858), B (RL2862), C (pRL110330), D (pRL100104), E (pRL100107) and $F$ (pRL100120). Pool-Seqs are indicated as $P_{A}\left(\right.$ pea $\left._{A}\right), P_{B}\left(\right.$ pea $\left._{B}\right), L_{A}\left(\right.$ lentil $\left.A_{A}\right), L_{B}\left(\right.$ lentil $\left.{ }_{B}\right), F_{A}$ (fava bean $\left.{ }_{A}\right), F_{B}\left(\right.$ fava bean $\left.n_{B}\right), V_{A}$ $\left(\right.$ vetch $\left._{A}\right), V_{B}\left(\right.$ vetch $\left._{B}\right), P$ (initial pea), L (initial lentil), $F$ (initial fava bean), $V$ (initial vetch) and $S$ (soil). Plant hosts were colour-coded (see Figure 3.1), and soil was coded orange. Initial host and mesocosm samples A and B are indicated as circles, triangles, and squares, respectively. * indicates highly significant $F_{\text {ST }}$ values (over 0.25 ). Circles group subpopulations with no significant $F_{\mathrm{ST}}$ among them. White bars within a subpopulation symbol indicate a low coverage value (below 10 BPKM) for at least one gene in the region analysed. 
pRL100104: Four genotypes were observed, one for pea $_{\mathrm{A}}$, another for vetch mesocosm and pea $a_{\mathrm{B}}$, one that grouped together the soil and all the lentil Pool-Seqs, and a group including all fava bean subpopulations plus initial pea and vetch (Figure $3.16 \mathrm{C})$.

pRL100107: Five groups were distinguished, group I: initial host subpopulation plus fava bean mesocosm Pool-Seqs, group II: lentil mesocosm subpopulations, group

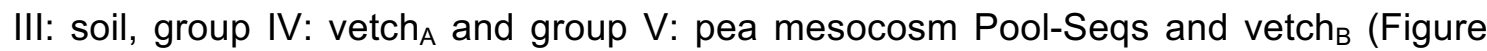
$3.16 \mathrm{D})$.

pRL100120: three groups were established, group I: pea and vetch mesocosm Pool-Seqs; group II: initial host-selected subpopulations plus fava bean mesocosm Pool-Seqs and lentil ${ }_{B}$; and group III: soil. Lentil ${ }_{A}$ clustered with lentil ${ }_{B}$, but not with the other group II subpopulations (Figure 3.16 E).

\subsubsection{Individual genomic analysis}

Many of the population genomics results presented above would benefit from genomic analysis of individual strains isolated from mesocosm experiments. A small subset of representative strains (one for each mesocosm sample) was selected and their genomes sequenced, assembled, annotated and comparatively studied. The analyses presented below were restricted to those aspects relevant to our research question and do not include a thorough comparative genomic characterization of these strains that, although quite interesting in itself, falls outside the scope of this Thesis.

\subsubsection{Selection of genotypes}

Two fragments, one from the $r p o B(447 \mathrm{nt})$, and the other from the nodC (523 nt) genes, were amplified and sequenced for each of 200 host Rhizobium leguminosarum isolates (25 isolates from each of the eight mesocosm Pool-Seqs; see Table M4 from Material and methods). A multiple alignment was constructed for each gene, and Neighbor-joining phylogenies were derived from them. Different $r p o B$ and nod $C$ genotypes were defined from the total 200 strains, and these genotypes were colourcoded and presented in schematic form in Figure 3.17. Also shown are the different relative abundances of each genotype in mesocosm subpopulations (reflected by different bar sizes). One strain was selected for further study from the most abundant genotype found in each mesocosm subpopulation (marked with a yellow asterisk): GPS1_03, GPS2_15, LPS1_35, HPS1_09, HPS2_12, VPS1_17 and VPS2_25 for pea $_{A}$, pea $a_{B}$, lentil $A_{A B}$, fava bean $n_{A}$, fava bean ${ }_{B}$, vetch ${ }_{A}$ and vetch $_{B}$, respectively. Due to the high number of genotypes found for lentil mesocosm Pool-Seqs, only one genotype 
was selected for both subpopulation, the most abundant and exclusive genotype uncovered in lentil $\left.\right|_{A}$, represented by strain LPS1_35. It is worth noting that for both pea $_{B}$

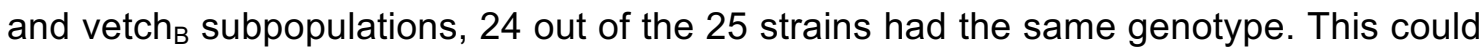
be interpreted as evidence that one preferred genotype was selected during the mesocosm experiment, at least for $r p o B$ and nodC.

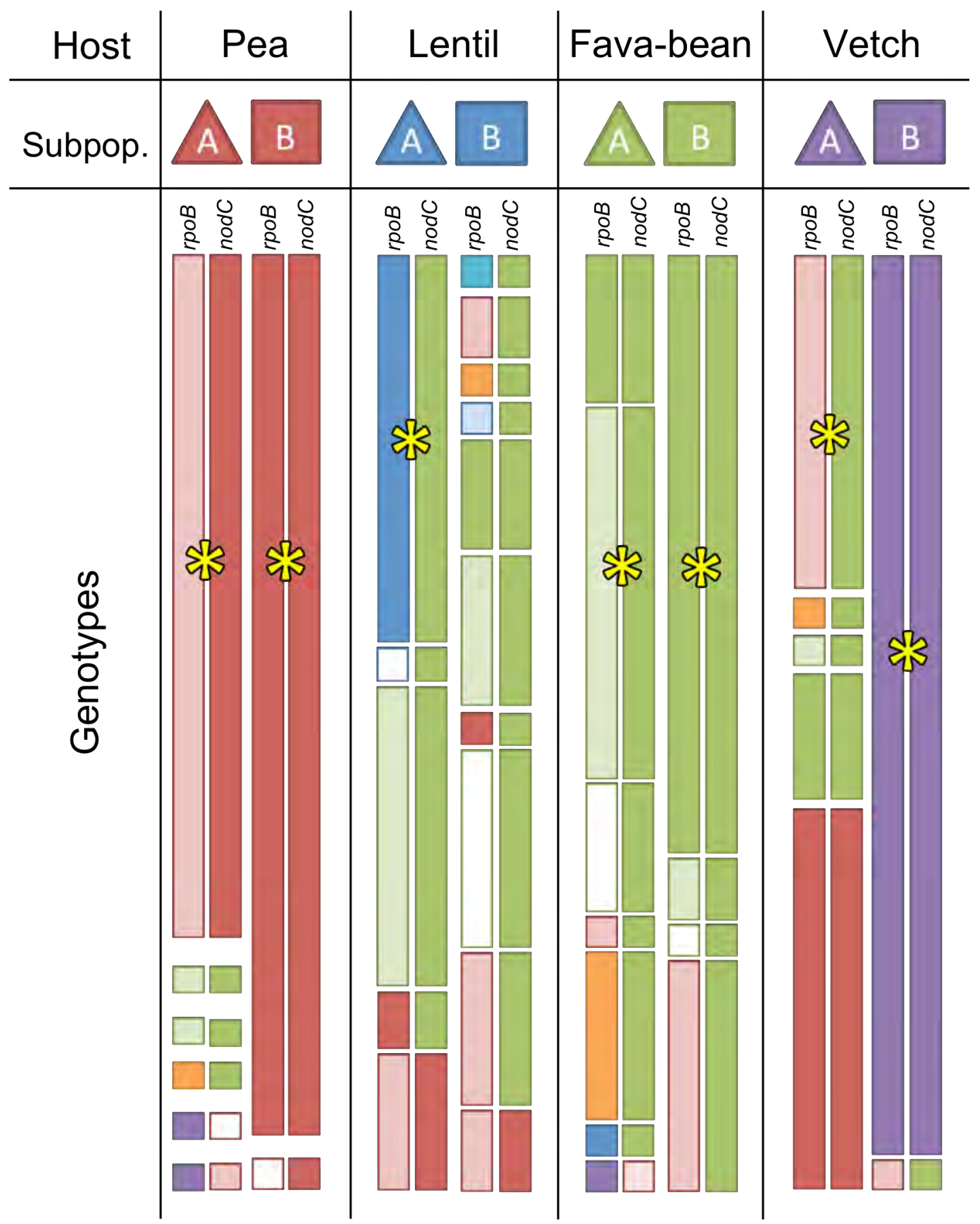

Figure 3.17. $r p o B$ and $n o d C$ mesocosm genotypes. Schematic representation of different $r p o B$ and nod $C$ genotypes found in the mesocosm subpopulations. For each gene, each colour and shade represent different genotypes (with, at least, one nucleotide difference). Plant-enriched or plant-characteristic genotypes were assigned plant specific colours (see Figure 3.1). The size of the bars is proportional to the relative number of strains that have each genotype. Yellow stars indicate the genotypes of mesocosm-selected strains chosen for further study. 


\subsubsection{Cross-inoculation assay and nitrogenase activity}

A cross-inoculation assay was conducted for the seven selected strains (GPS1_03, GPS2_15, LPS1_35, HPS1_09, HPS2_12, VPS1_17 and VPS2_25) in each of the four hosts ( $P$. sativum, $L$. culinaris, $V$. faba, $V$. sativa). The presence and colour of nodules was recorded, and nodule nitrogenase activity was measured. Results are presented in Table 3.6. Symbiotic differences were observed with V. faba plants. Strains GPS1_03, GPS2_15 (selected from pea mesocosm Pool-seqs) and VPS2_25 (selected from vetch $_{B}$ subpopulation) were either unable to form nodules or presented non-functional (white) nodules; causing nitrogenase activity to be absent. For LPS1_35 no information was obtained because seedlings died after inoculation (only one replicate was performed).

Table 3.6. Cross inoculation results of the seven selected strains in four plant hosts

\begin{tabular}{ccccccccc}
\hline & \multicolumn{2}{c}{$P$. sativum } & \multicolumn{2}{c}{ L. culinaris } & \multicolumn{2}{c}{ V. faba } & \multicolumn{2}{c}{ V. sativa } \\
\hline strain & Nod. & $\mathrm{N}_{\text {ase }}$ Act & Nod. & $\mathrm{N}_{\text {ase }}$ Act & Nod. & $\mathrm{N}_{\text {ase }}$ Act & Nod. & $\mathrm{N}_{\text {ase }}$ Act \\
GPS1_03 & + & + & + & + & - & - & + & + \\
GPS2_15 & + & + & + & + & - & - & + & + \\
LPS1_35 & + & + & + & + & nd & nd & + & + \\
HPS1_09 & + & + & + & + & + & + & + & + \\
HPS2_12 & + & + & + & + & + & + & + & + \\
VPS1_17 & + & + & + & + & + & + & + & + \\
VPS2_25 & + & + & + & + & - & - & + & + \\
\hline Nod. (presence of nodules). $\mathrm{N}_{\text {ase }}$ Act (Nitrogenase activity). nd (no data) & & &
\end{tabular}

\subsubsection{Next generation sequencing of mesocosm-selected strains}

DNA from individual cultures of each mesocosm-selected strain was submitted to next generation sequencing (Illumina MiSeq v.3, 600 bp PE libraries, 300 bp reads, from 0.6 to 1.3 Mreads; ASU Genomic Core, Tempe, AZ). On average, each genome was sequenced to a coverage of 24-50x. Individual reads were quality trimmed, assembled and functionally annotated (see Materials and Methods). General features of the assembly results are described in Table 3.7. All the genomes were assembled at draft level, with numbers of contigs assembled ranging from 56 to 229 . However, if only contigs larger than $500 \mathrm{bp}$ were considered, these numbers dropped to 27-127. Besides GPS1_03, with an estimated genome size of $8.45 \mathrm{Mb}$, the remaining six strains had genome sizes ranging from 7.19 to $7.85 \mathrm{Mb}$, quite coincident with the range of sizes of $R$. leguminosarum bv. viciae reference genomes (from 7.66 to $7.75 \mathrm{Mb}$ ) 
Table 3.7. Genome sequence assembly statistics for the seven selected strains

\begin{tabular}{ccccccc}
\hline Strain & $\begin{array}{c}\text { Total } \\
\text { length }\end{array}$ & $\begin{array}{c}\text { No. } \\
\text { Contigs }\end{array}$ & $\begin{array}{c}\text { No. Contigs } \\
(>500 \mathrm{bp})\end{array}$ & $\begin{array}{c}\text { Largest } \\
\text { Contig }\end{array}$ & $\begin{array}{c}\% \\
\text { N's }\end{array}$ & GC (\%) \\
\hline GPS1_03 & $8,452,486$ & 229 & 127 & $5,080,721$ & 0.089 & 60.70 \\
GPS2_15 & $7,797,102$ & 208 & 111 & $4,989,166$ & 0.038 & 60.84 \\
LPS1_35 & $7,436,067$ & 201 & 77 & $4,958,051$ & 0.056 & 61.03 \\
HPS1_09 & $7,411,578$ & 313 & 120 & $5,093,793$ & 0.087 & 60.85 \\
HPS2_12 & $7,191,761$ & 56 & 27 & $5,041,188$ & 0.081 & 60.95 \\
VPS1_17 & $7,851,993$ & 113 & 43 & $5,012,517$ & 0.103 & 60.77 \\
VPS2_25 & $7,738,392$ & 162 & 58 & $5,181,985$ & 0.190 & 60.61 \\
\hline
\end{tabular}

$\%$ N's (percentage of mismatches)

previously described in the present Thesis (see Table 2.11 from Chapter 2). Other, larger genomes have been described in the $R$. leguminosarum group, such as $R$. leguminosarum bv. trifolii TA1 (8.61Mb), or R. leguminosarum bv. trifolii WSM2297 $(7.99 \mathrm{Mb})$. Despite the high number of contigs, some large contigs (from 4.96 to 5.18 $\mathrm{Mb}$ ) could be assembled; these contigs have sizes similar to the chromosome of the closed reference genomes (from 4.76 to $5.67 \mathrm{Mb}$ ). Furthermore, the observed $\mathrm{G}+\mathrm{C}$ contents for all the mesocosm-selected strains were in the same range as the Rhizobium leguminosarum bv. viciae reference genomes, between 60-61\% (Young et al., 2006, Reeve et al., 2015) (Sanchez-Cañizares et al, in preparation). Basic statistics were calculated after annotation (Table 3.8): the number of genes correlated with the total length of the draft genome, where GPS1_03 had 8,103 genes and HPS2_12 6,875 genes. This gene content difference could be explained through the presence of a plasmid or plasmids in GPS1_03 that was / were absent in HPS2_12, or due to different plasmids sizes. It is also noteworthy that a diverse number of rRNA genes were annotated within the host-selected, mesocosm genomes. Rhizobium leguminosarum bv viciae strains usually have three copies of 16S-23S-5S rRNA genes, which are almost identical (personal observation). This made it quite difficult to assemble them separately.

Table 3.8. Genome statistics for the seven selected strains

\begin{tabular}{ccccc}
\hline Strain & No. genes & No. CDS & rRNA & tRNA \\
\hline GPS1_03 & 8,103 & 8,048 & 3 & 51 \\
GPS2_15 & 7,474 & 7,424 & 4 & 45 \\
LPS1_35 & 7,099 & 7,045 & 3 & 50 \\
HPS1_09 & 7,103 & 7,053 & 5 & 44 \\
HPS2_12 & 6,875 & 6,821 & 6 & 47 \\
VPS1_17 & 7,490 & 7,436 & 3 & 50 \\
VPS2_25 & 7,351 & 7,299 & 3 & 48 \\
\hline
\end{tabular}




\subsubsection{Mesocosm-selected strains adequately represent their corresponding Pool-} Seq subpopulations

In order to ensure that mesocosm-selected strains were good representatives of their mesocosm subpopulations, reads from each mesocosm Pool-Seq were aligned against the assembled genomes. Table 3.9 summarizes these results. All the mesocosm selected-strains were able to recruit at least $76 \%$ of the Pool-Seq reads, which means that at least $76 \%$ of the population genome was included in the mesocosm-selected genomes. For some strains, read recruitment ratios were remarkably high. This was the case of GPS2_15 (99.75\%) and VPS2_25 (98.34\%), and suggests that the pea $_{B}$ and vetch ${ }_{B}$ Pool-Seqs contained a single strain each. Likewise, SNPs variation was very low for both subpopulations, although it was not zero. Sequencing and recruitment errors inherent to the methodologies used could contribute to this low level of polymorphism, or else it is possible that the Pool-Seq subpopulations (constituted by 25 isolates each) were not absolutely clonal. In any case, the mesocosm-selected strains were clearly representative of the genotypes of these plant-host selected subpopulations.

Table 3.9. Comparison of mesocosm host-specific Pool-Seqs with their corresponding hostselected reference genome

\begin{tabular}{cccc}
\hline Pool-Seq & Ref. Genome $^{*}$ & \% reads recruited & \% SNPs \\
\hline Pea $_{A}$ & GPS1_03 & 78.39 & 0.838 \\
Pea & GPS2_15 & 99.75 & 0.005 \\
Lentil $_{A}$ & LPS1_35 & 83.25 & 2.769 \\
Lentil $_{B}$ & LPS1_35 & 81.02 & 3.878 \\
Fava bean & HPS1_09 & 76.89 & 2.965 \\
fava bean $_{B}$ & HPS2_25 & 88.60 & 3.043 \\
Vetch $_{A}$ & VPS1_17 & 82.22 & 2.645 \\
Vetch $_{B}$ & VPS2_25 & 98.34 & 0.052 \\
\hline${ }^{*}$ Ref.: reference & & &
\end{tabular}

\subsubsection{Average Nucleotide Identity (ANI) of mesocosm-selected strains}

Genomic homology among mesocosm-selected strains, including Rhizobium leguminosarum bv. viciae complete reference genomes, was estimated by calculating the Average Nucleotide Identity (ANI). Genomic sequences were used for ANIMUMmer (ANIm) calculations with the JSpecies package (Richter and Rosselló-Móra, 2009). Distances were calculated as 1-ANIm and trees were computed by the UPGMA method (Sneath and Sokal, 1973). The resulting tree is shown in Figure 3.18. The selected mesocosm strains were clustered in three different sets: group I (HPS1_09 and HPS2_12), group II (GPS1_03, VPS1_17; LPS1_35, GPS2_15 and $R$. 
leguminosarum bv. viciae 3841) and group III (VPS2_25 and R. leguminosarum bv. viciae UPM791). Both plant host-selected strains clustered together for fava bean (group I) and pea (group II). VPS2_25 (group III) was the most distant strain when all mesocosm-selected strains were considered together (Figure 3.21).

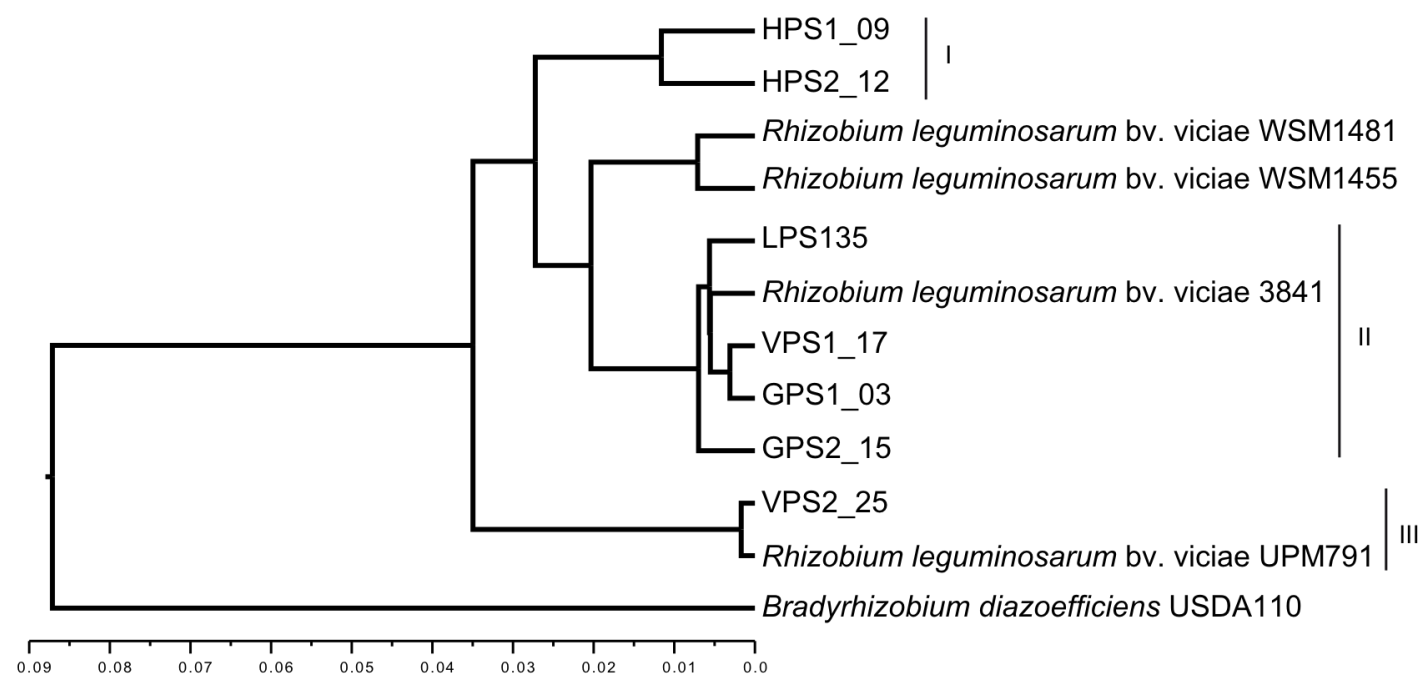

Figure 3.18. Genomic similarity among mesocosm-selected strains. Distances were calculated as 1-ANIm and the tree was computed by the UPGMA method using the MEGA 6 package. The resulting optimal tree is drawn to scale, with branch lengths in the same units as those of the evolutionary distances used to infer the phylogenetic tree. Groups are indicated as I-III.

\subsubsection{Symbiotic clusters: Synteny and diversity}

Synteny and phylogenetic diversity were studied for relevant symbiotic-related gene clusters: nif (fdxB-nifNEKDH, Figure 3.19), rhi (rhil-ABCRD, Figure 3.20), nod (nodO(T)NMLEFDABCIJ, Figure 3.21), nif-fix (nifBA-fixXCBA, Figure 3.22) and fix (fixNOQPGHIS-Transcriptional Factor, Figure 3.23). For each cluster, nucleotide regions were extracted, aligned using Muscle algorithm (Edgar, 2004) and Neighborjoining phylogenies were derived from them.

nif cluster. A high synteny was observed among genomes, despite the absence of a hypothetical protein present in $R$. leguminosarum bv. viciae 3841 between $f d x B$ (ferredoxin) and nifN (Figure 3.19, panel A), although this could be due to issues with the annotation algorithms. Three different clusters were observed: group I (LPS1_35, VPS1_17 and R. leguminosarum bv. viciae 3841), group II (R. leguminosarum bv. viciae UPM791 and HPS2_12) and group III (HPS1_09, VPS2_25, GPS1_03 and GPS2_15). Except for HPS1_09, the same results were observed in nif cluster PoolSeq comparisons (Figure 3.13, panel B). It is possible that HPS1_09 did not represent fava bean $_{A}$ diversity for nif genes, perhaps being a minor component of this 
subpopulation. Two possible explanations can be advanced for this hypothesis: 1) the Pool-Seq restriction to recruit only those reads with an identity value above $85 \%$ could

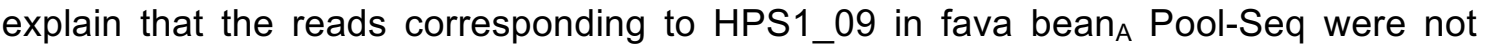
aligned to nif cluster, or 2) HPS1_09 reads did align to the reference genome, but its exclusive SNPs were below the SNPs frequency threshold $(0.1)$ and therefore

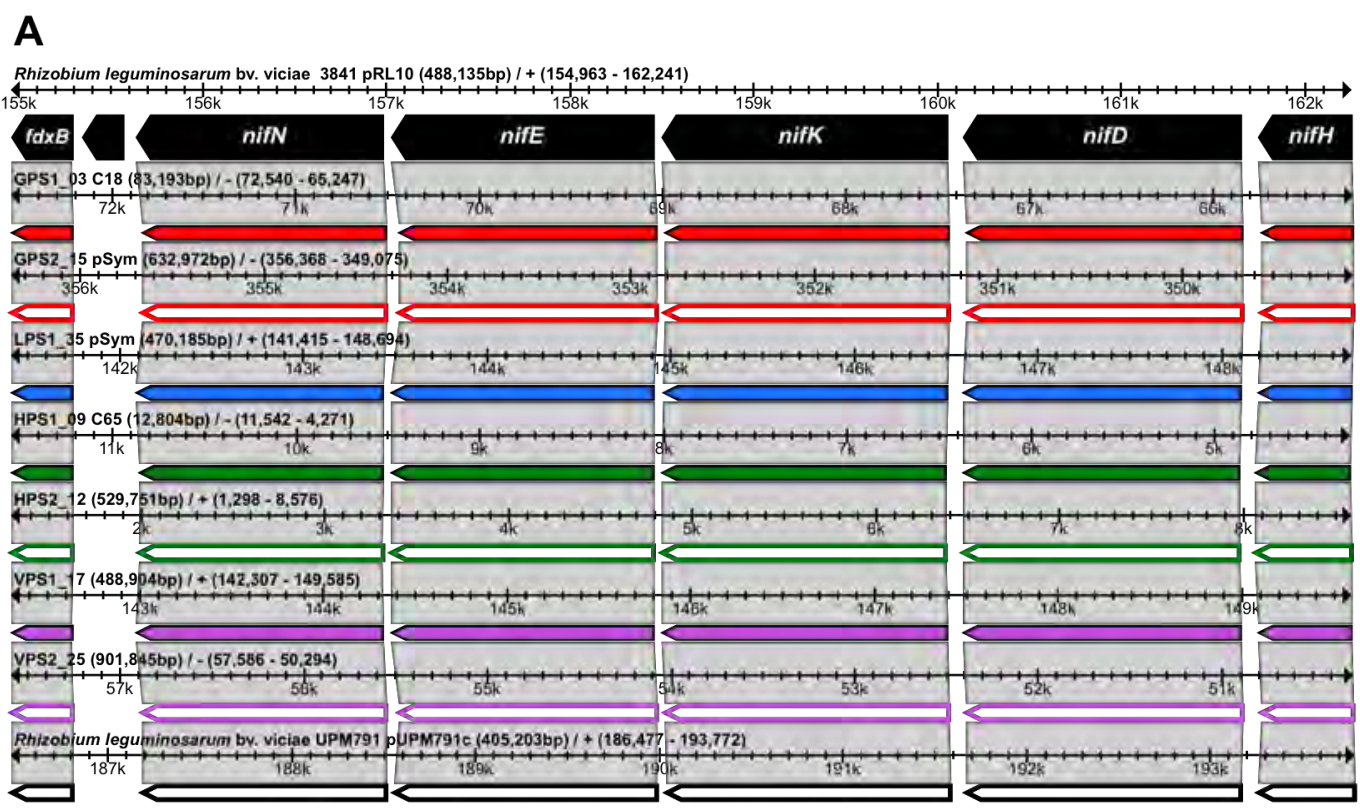

B

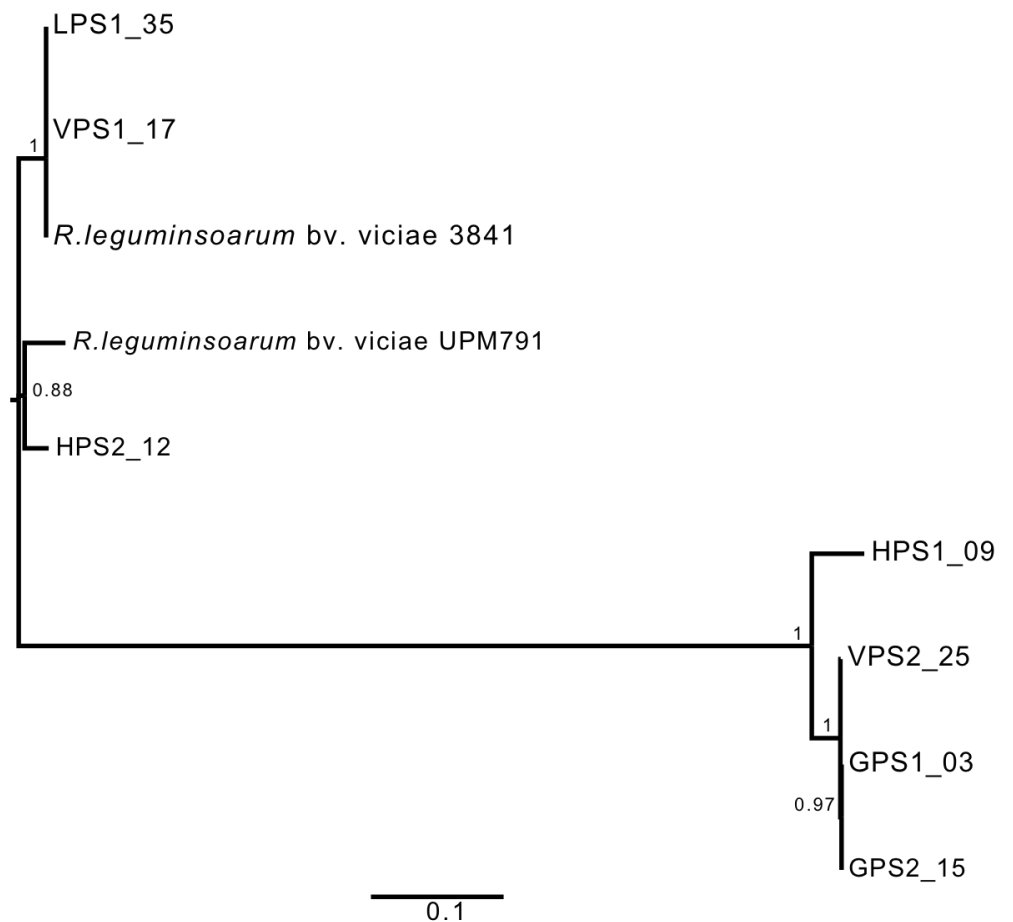

Figure 3.19. Genomic organization, synteny and diversity of nif cluster. Panel A compares synteny and genomic organization of mesocosm-selected strains and the reference genomes Rhizobium leguminosarum bv. viciae 3841 and $R$. leguminosarum bv. viciae UPM791. Panel B presents the phylogenetic tree based on the multiple alignment of the complete nif region. The tree was constructed by the neighbor-joining method. The significance of each branch is indicated by a bootstrap value calculated for 1,000 subsets. 
discarded. These two possibilities were tested by recruiting HPS1_09 genomic reads to the reference genome $R$. leguminosarum bv. viciae 3841 . The $R$. leguminosarum bv. viciae 3841 symbiotic region was covered by HPS1_09 genomic reads, which rules the first hypothesis out. The second hypothesis was tested by performing a $F_{\mathrm{ST}}$ analysis

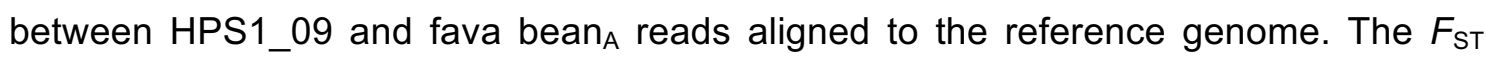
values for each symbiosis-related cluster were above 0.50 , which means that

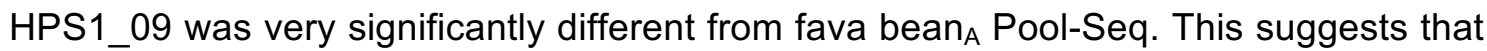

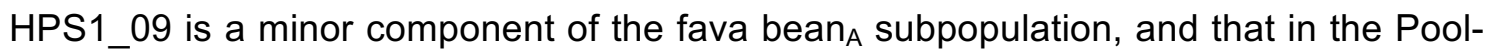
Seq analyses, the SNPs that were present in HPS1_09 genome had been discarded because their frequency (in the Pool-Seq) was below the 0.1 frequency threshold.

rhi cluster. The main differences among genomes were located in the intergenic region between rhil and rhiA. LPS1_35 and VPS1_17, like the reference strain $R$. leguminosarum bv. viciae 3841, presented two transposases of the IS4 family (Figure 3.20, panel A). The other reference strain, $R$. leguminosarum bv. viciae UPM791, presented an inversion of rhiD, which suggests that the rhi region is highly variable. Three different clusters were defined for the rhi region in terms of diversity: group I (LPS1_35, VPS1_17, HPS2_12 and R. leguminosarum bv. viciae 3841), group II (VPS2_25, GPS1_03 and GPS2_15) and group III (R. leguminosarum bv. viciae UPM791 and HPS1_09) (Figure 3.20, panel B). 
A

Rhizobium leguminosarum bv. viciae 3841 pRL10 (488,135bp) / + (165,376 - 173,943)

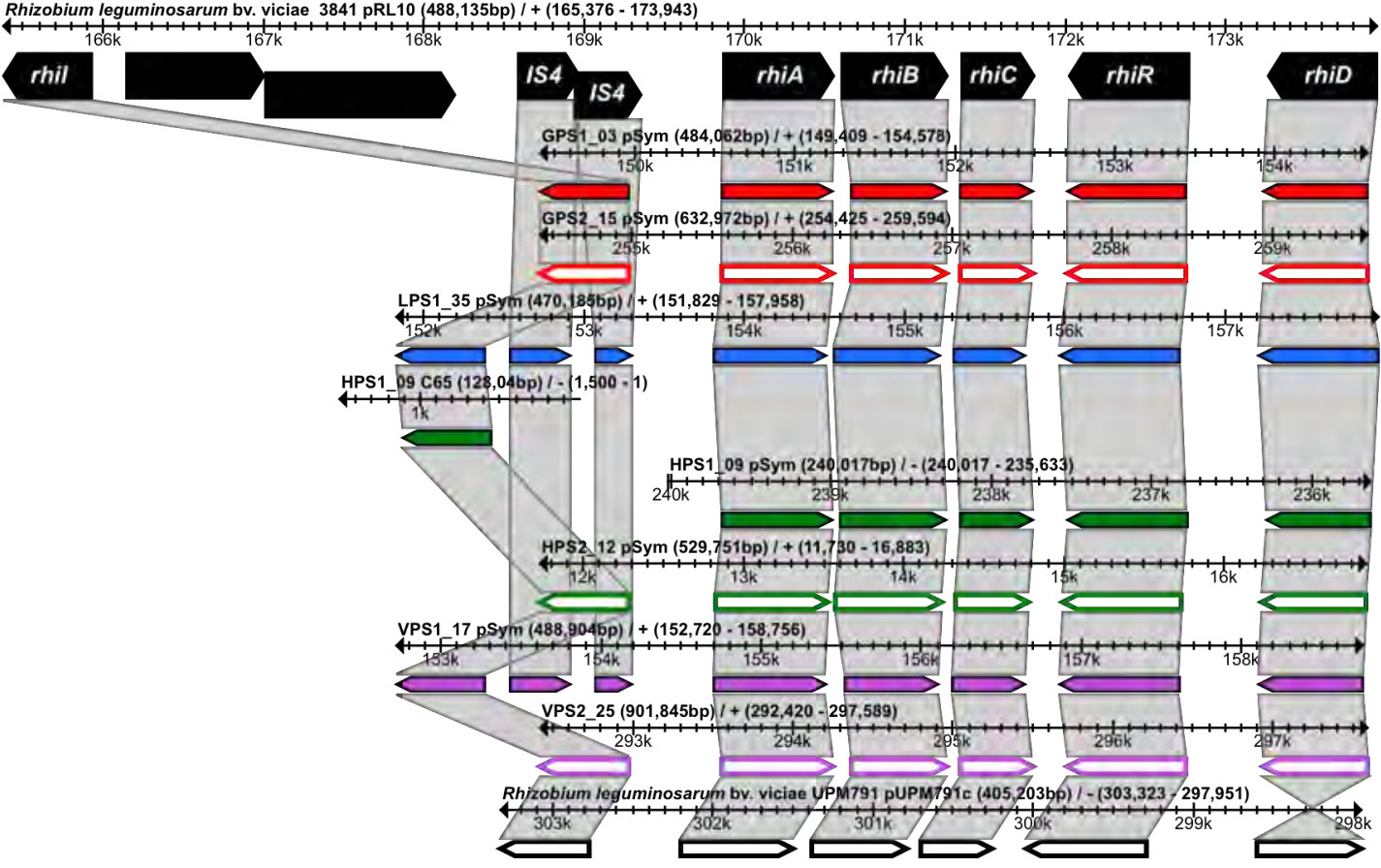

B

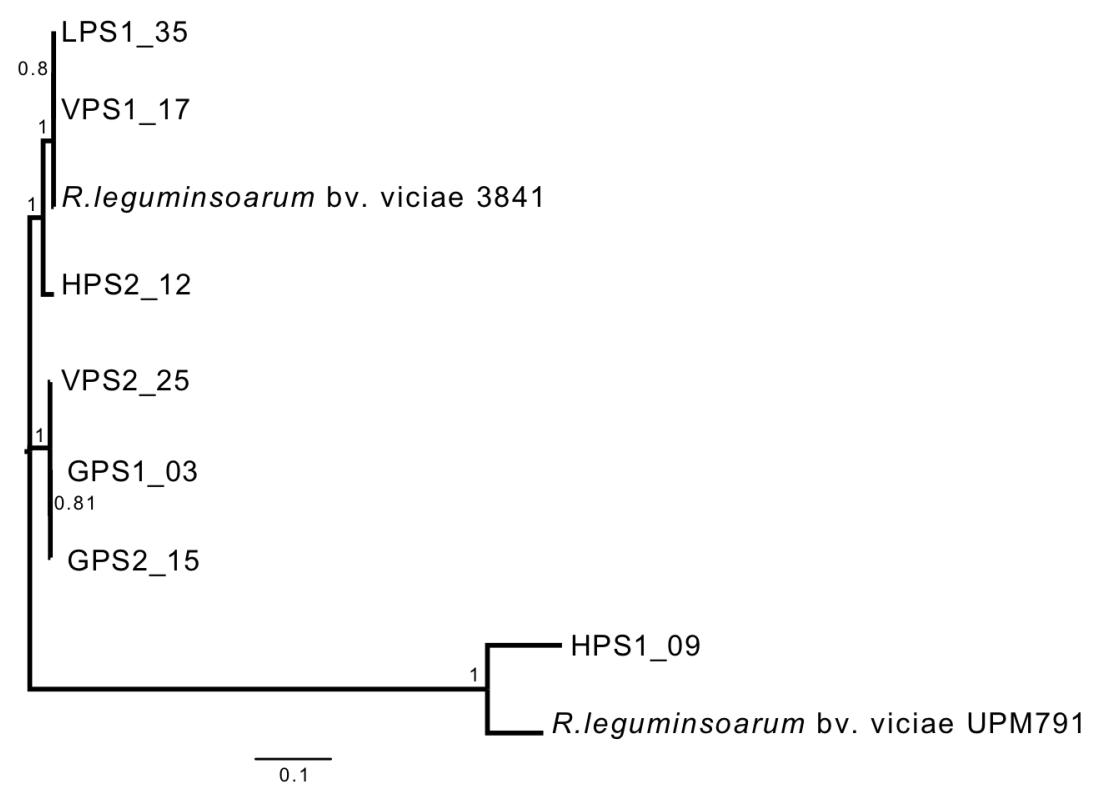

Figure 3.20. Genomic organization, synteny and diversity of rhi cluster. Panel A compares synteny and genomic organization of mesocosm-selected strains and the reference genomes Rhizobium leguminosarum bv. viciae 3841 and $R$. leguminosarum bv. viciae UPM791. Panel $\mathrm{B}$ presents the phylogenetic tree based on the multiple alignment of the complete rhi region. The tree was constructed by the neighbor-joining method. The significance of each branch is indicated by a bootstrap value calculated for 1,000 subsets. 
nod cluster. Synteny alterations were found in the intergenic regions nodO-nodT and nodF-nodD (Figure 3.21, panel A). Between nodO and nodT, two genes were predicted, the first one was annotated either as a hypothetical protein (GPS1_03, GPS2_15 and VPS2_25), as an IstB-like ATP binding protein (LPS1_35, VPS1_17, HPS2_12 and HPS1_09) or no description in R. leguminosarum bv. viciae 3841. The second gene was described as a hypothetical protein for all mesocosm-selected strains, and as a putative homologue for eukaryotic tubulin in $R$. leguminosarum bv. viciae 3841. $R$. leguminosarum bv. viciae UPM791 lacked both nodT and this intergenic region altogether. Differences were observed in the size of the intergenic region between nodF and nodD (Table 3.10). In GPS1_03, GPS2_15 and VPS2_25 it consisted of 666bp, while in LPS1_35, HPS1_03 and HPS2_12 it was 644-645bp. Strain VPS1_17 had a 1,677 bp nodF-nodD intergenic region that encoded a predicted hypothetical protein. At the level of diversity, three different groups, coincident with those described for the rhi cluster, were defined (Figure 3.21, panel B).

Table 3.10. nodF- $D$ intergenic region sizes.

\begin{tabular}{cccc}
\hline Strain & Start & End & Size \\
\hline RIv3841 & 184,712 & 185,356 & 644 \\
RIv UPM791 & 281,233 & 280,593 & 640 \\
GPS1_03 & 165,654 & 166,320 & 666 \\
GPS2_15 & 270,670 & 27,1336 & 666 \\
LPS1_35 & 168,660 & 169,304 & 644 \\
HPS1_09 & 224,425 & 223,780 & 645 \\
HPS2_12 & 28,090 & 28,735 & 645 \\
VPS1_17 & 169,551 & 171,228 & 1,677 \\
VPS2_25 & 308,665 & 309,331 & 666 \\
\hline
\end{tabular}


A

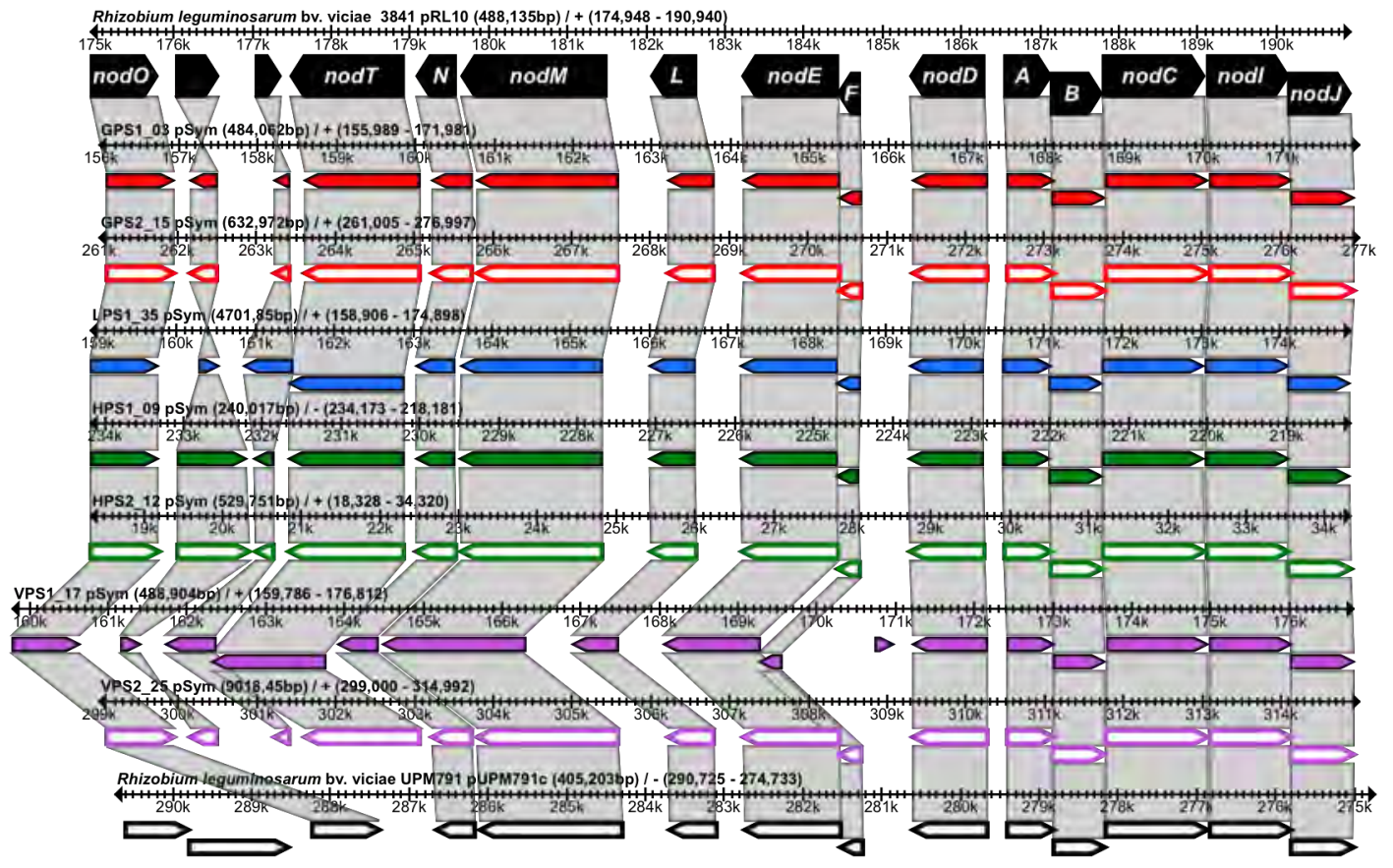

B

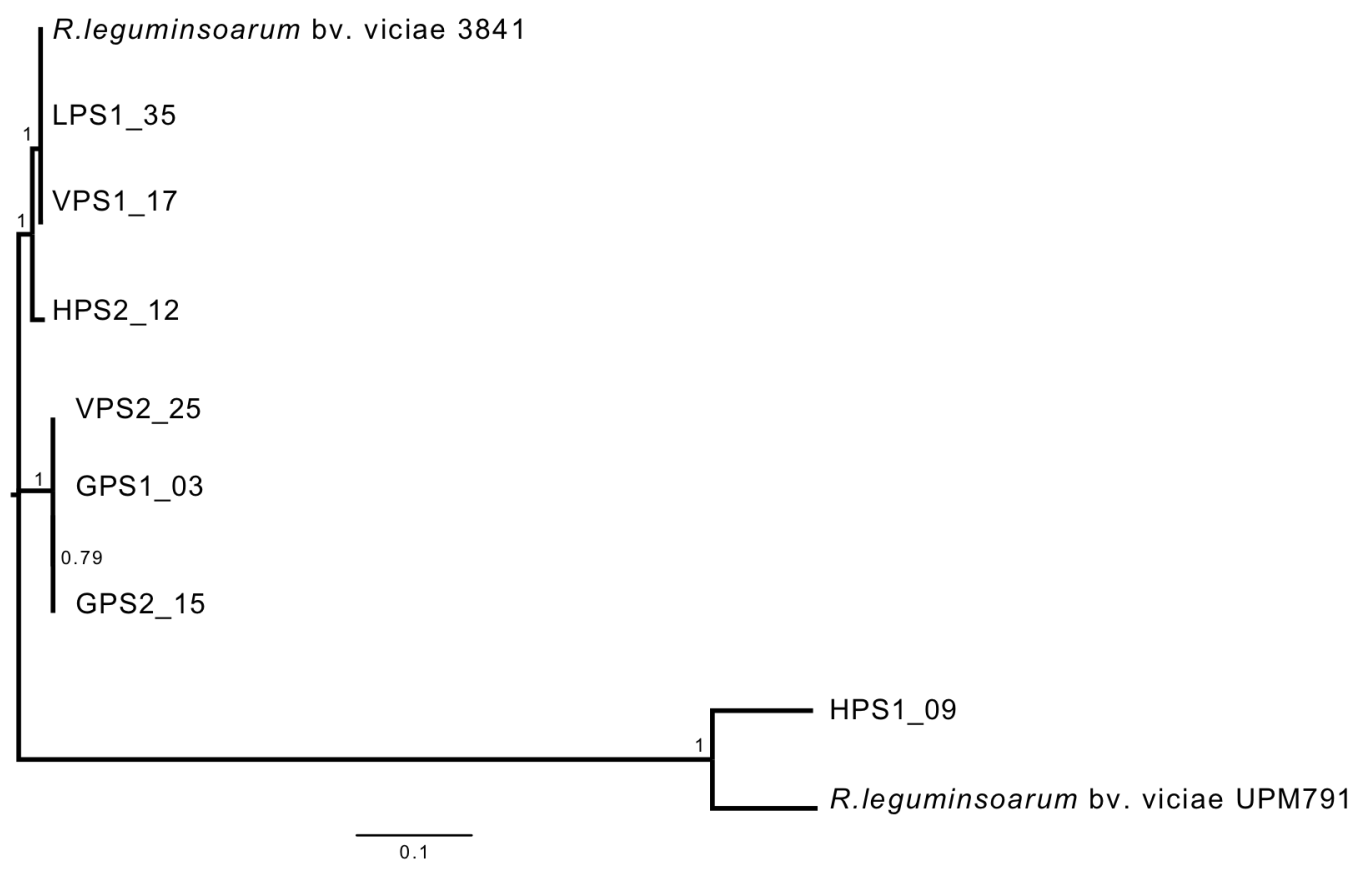

Figure 3.21. Genomic organization, synteny and diversity of nod cluster. Panel A compares synteny and genomic organization of mesocosm-selected strains and the reference genomes Rhizobium leguminosarum bv. viciae 3841 and $R$. leguminosarum bv. viciae UPM791. Panel B represents the phylogenetic tree based on the multiple alignment of the complete nod region. The tree was constructed by the neighbor-joining method. The significance of each branch is indicated by a bootstrap value calculated for 1,000 subsets 
nif-fix cluster. No remarkable differences were found at the level of synteny. However four groups were defined when the diversity was studied: group I (LPS1_35, VPS1_17 and R. leguminosarum bv. viciae 3841), group II (HPS1_12), group III (VPS2_25, GPS1_03 and GPS2_15) and group IV (R. leguminosarum bv. viciae UPM791 and HPS1_09) (Figure 3.22).

A

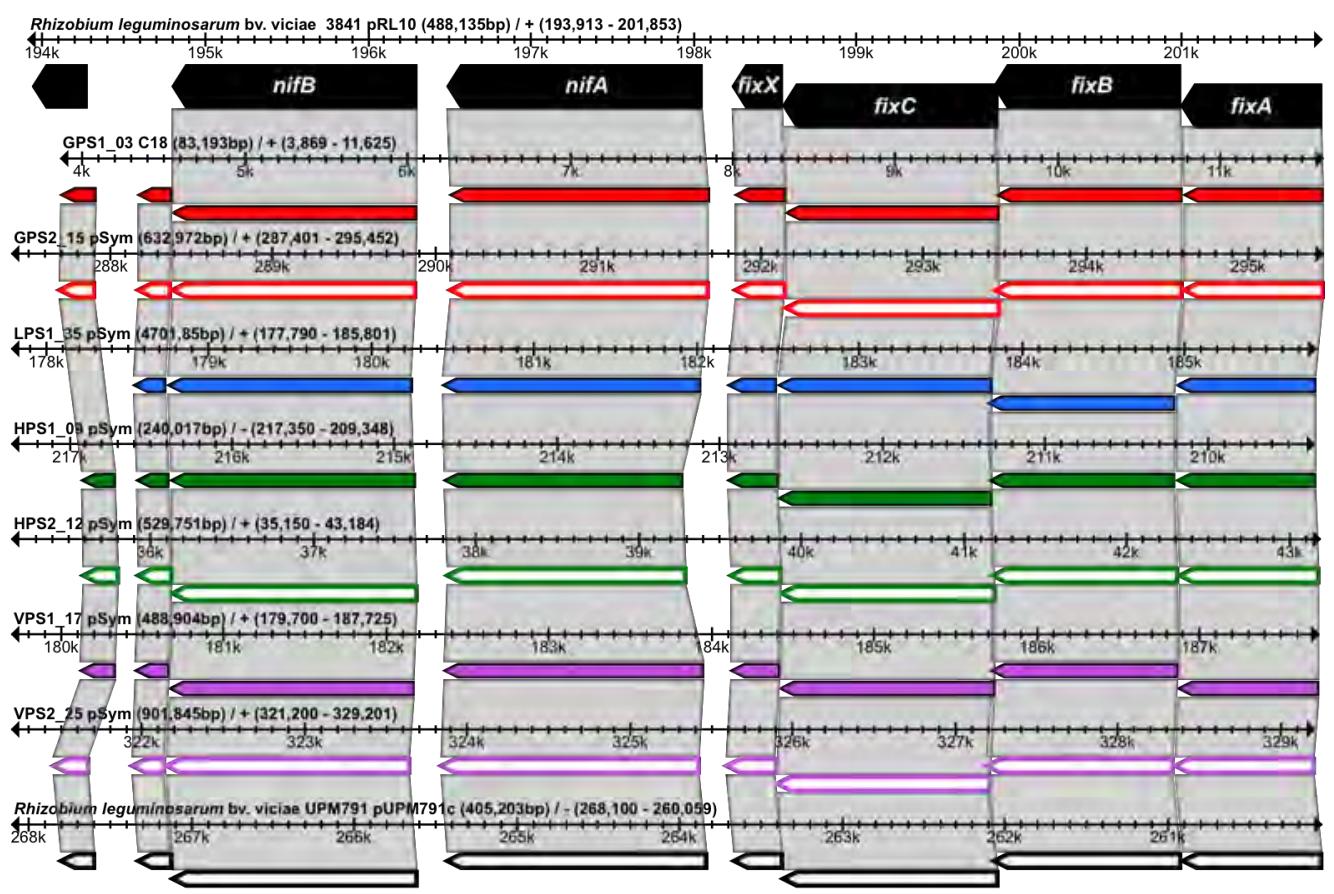

B

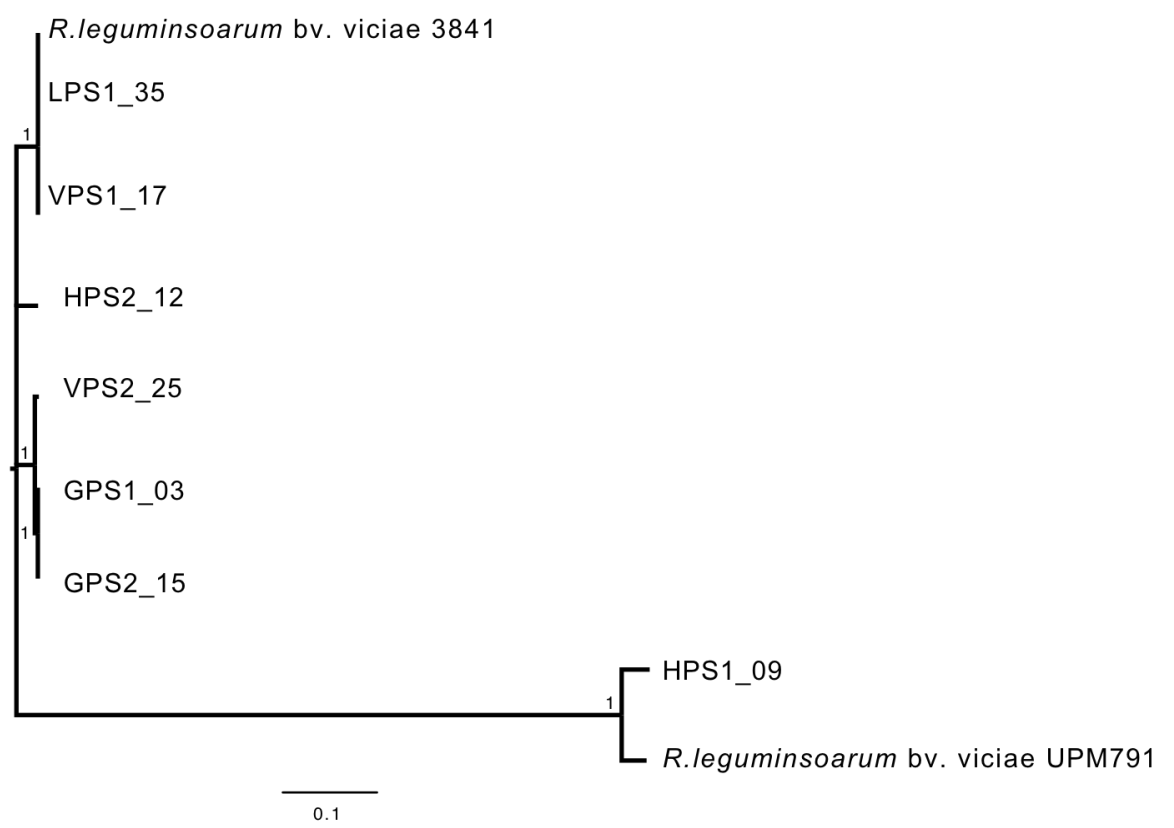

Figure 3.22. Genomic organization, synteny and diversity of nif-fix cluster. Panel A compares synteny and genomic organization of mesocosm-selected strains and the reference genomes Rhizobium leguminosarum bv. viciae 3841 and $R$. leguminosarum bv. viciae UPM791. Panel $B$ represents the phylogenetic tree based on the multiple alignment of the complete nif-fix region. The tree was constructed by the neighbor-joining method. The significance of each branch is indicated by a bootstrap value calculated for 1,000 subsets. 
fix cluster. Two main differences were observed (Figure 3.23). VPS1_17 showed a remarkably larger intergenic region between fix $Q-P$ due to a presence of gap between features, which made it impossible to annotate correctly this region. Two different transcriptional factors were annotated downstream fixS; GPS1_03, LPS1_35, HPS1_09 and HPS2_12, together with reference strain $R$. leguminosarum bv. viciae 3841 presented a CRP/FNR family transcriptional regulator. However, GPS2_15 and VPS2_25, together with reference strain $R$. leguminosarum bv. viciae UPM791 had a CDS annotated as RNA polymerase $\sigma 54$ (transcriptional regulator). 
A

Rhizobium leguminosarum bv. viciae 3841 pRL10 (488, 135bp) / + (206,260 - 215,216)

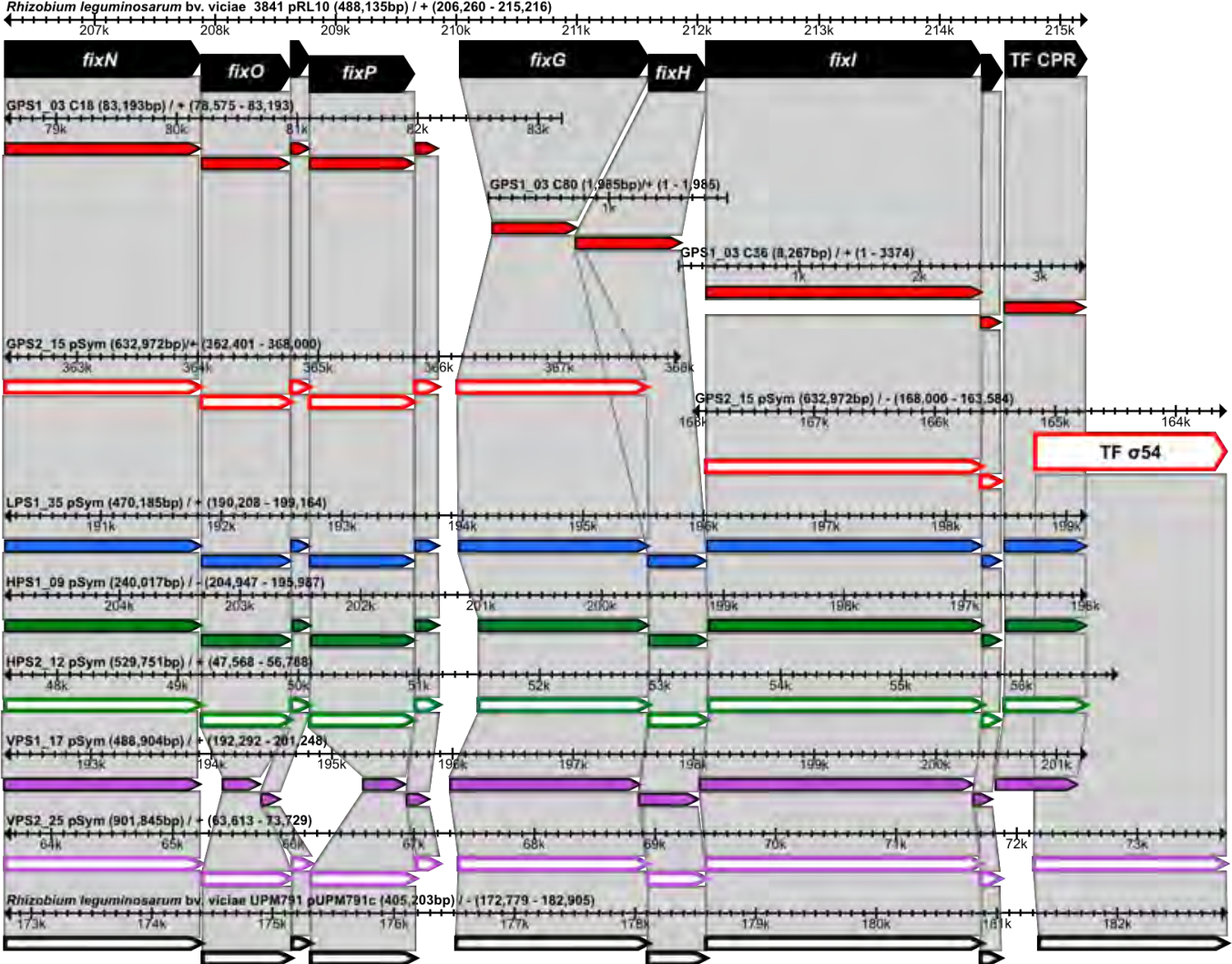

B

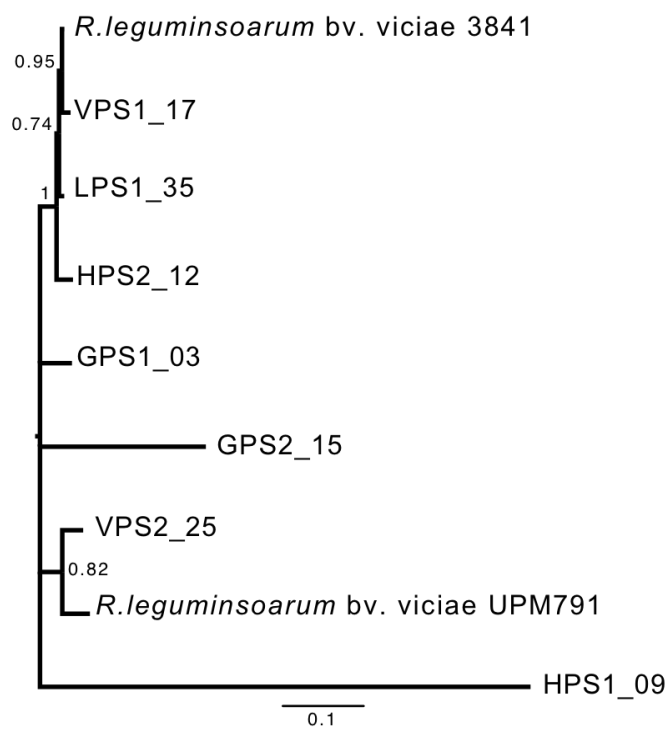

Figure 3.23. Genomic organization, synteny and diversity of fix cluster. Panel A compares synteny and genomic organization of mesocosm-selected strains and the reference genomes Rhizobium leguminosarum bv. viciae 3841 and $R$. leguminosarum bv. viciae UPM791. Panel B represents the phylogenetic tree based on the multiple alignment of the complete fix region. The tree was constructed by the neighbor-joining method. The significance of each branch is indicated by a bootstrap value calculated for 1,000 subsets. 


\subsubsection{Symbiotic region organization}

A global analysis of the whole symbiotic region organization was conducted. Two different kinds of symbiotic region were present: a $R$. leguminosarum bv. viciae 3841 type, present in LPS1_35, HPS1_09, HPS2_12 and VPS1_17 (Figure 3.24); and a $R$. leguminosarum bv. viciae UPM791 type, present in GPS1_03, GPS2_15 and VPS2_25 (Figure 3.25). R. leguminosarum bv. viciae 3841 symbiotic region type presented the symbiosis-related gene clusters in the same region and in the same order and orientation: nif, rhi, nod, nif-fix and fix. R. leguminosarum bv. viciae UPM791 symbiotic region type is completely different, the symbiotic gene clusters were located in two separate regions, the first one with nif and fix clusters (Figure 3.25, panel A), and a second region with nif-fix, nod and rhi clusters (Figure 3.25, panel A). Regarding $R$. leguminosarum bv. viciae UPM791, an inversion was observed in the fix cluster for GPS1_03, GPS2_15 and VPS2_25. Likewise, the nif-fix nod rhi region $R$. leguminosarum bv. viciae UPM791, presented nine genes ( acyl-CoA dehydrogenase, $A B C$ transporter, alkanesulfonate monooxigenase, $F M N$ reductase and two hypothetical proteins) that were absent in the mesocosm-selected genomes (Figure 3.25, panel B).

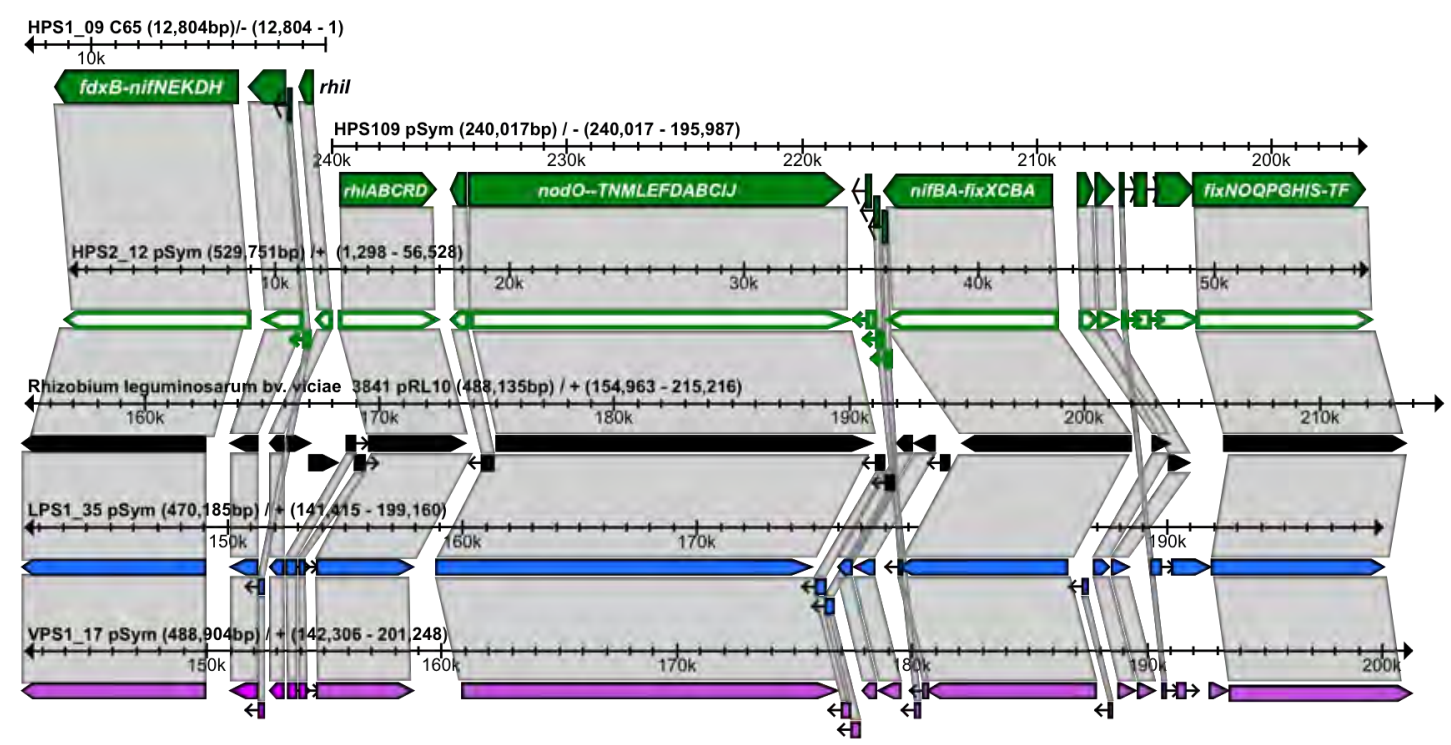

Figure 3.24. Genomic organization and synteny of the symbiotic region from Rhizobium leguminosarum bv. viciae 3841 type mesocosm-selected genomes (HPS1_09, HPS2_12, LPS1_35 and VPS1_17). 


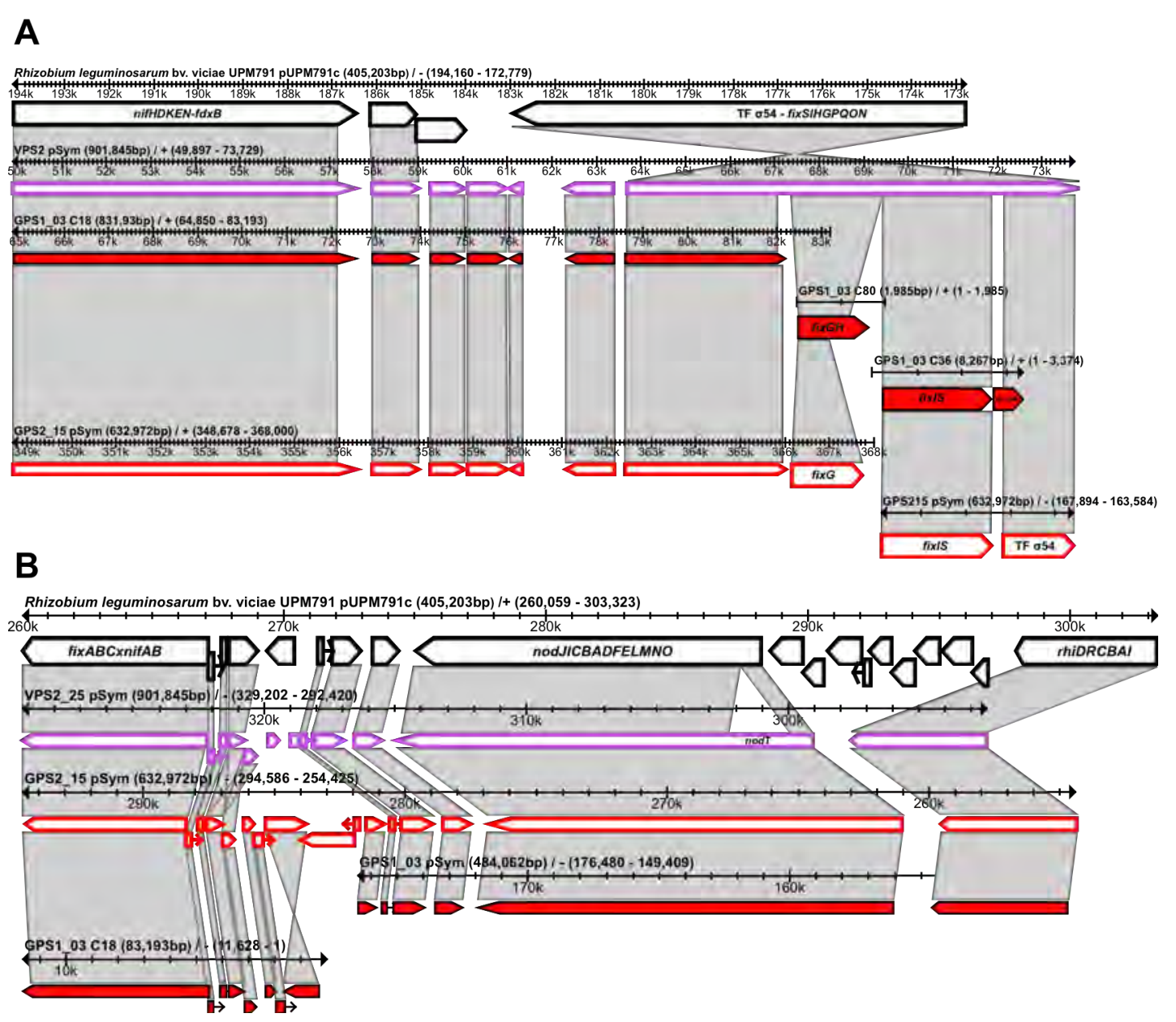

Figure 3.25. Genomic organization and synteny of the symbiotic region from Rhizobium leguminosarum bv. viciae UPM791 type mesocosm-selected genomes (GPS1_03, GPS2_15 and VPS2_25). Panel A: nif and fix regions; panel B: nif-fix, nod and rhi regions.

\subsubsection{Presence and diversity of "mesocosm-related" genes}

We also focused on genes whose presence and diversity had been suggested to be different in mesocosm host-selected populations on the basis of previous Pool-Seq analyses (Table 3.11). In addition to $R$. leguminosarum bv. viciae 3841 and $R$. leguminosarum bv. viciae UPM791, two more reference genomes were used, this time as outgroups: $R$. etli CFN42 and Ensifer meliloti 1021.

Commonly studied housekeeping genes (atpD, glnll, recA, rpoB), chromosomal "symbiosis-related" genes (rosR, fnrN), "rhizosphere" genes (cheA, fabF1, pssD and plyA) and differentially selected mesocosm genes (RL1858, RL2862, dctM and pRL110330) were all present in all mesocosm-selected strains, and similar phylogenetic relationships among genomes were inferred with any of these genes. Three strain clusters could be defined: group I (GPS1_03, GPS2_15, LPS1_35, VPS1_17 and R. leguminosarum bv. viciae 3841), group II (HPS1_09 and HPS2_12), and group III (VPS2_25 and R. leguminosarum bv. viciae UPM791) (Supplementary 
Chapter 3. Results

Table 3.11. Presence (grey) and absence (white) of mesocosm-related genes.

\begin{tabular}{|c|c|c|c|c|c|c|c|c|c|c|c|}
\hline & G1_03 & G2_15 & L1_35 & H1_09 & H2_25 & V1_17 & V2_25 & Em & $\operatorname{Re}$ & Rlv791 & Rlv3841 \\
\hline $\begin{array}{c}\text { atpD } \\
\text { (RL4407) }\end{array}$ & & & & & & & & & & & \\
\hline $\begin{array}{c}\text { glnll } \\
\text { (RL3549) }\end{array}$ & & & & & & & & & & & \\
\hline $\begin{array}{c}\text { recA } \\
(\mathrm{RL} 2637)\end{array}$ & & & & & & & & & & & \\
\hline $\begin{array}{c}\text { rpoB } \\
\text { RL1766 }\end{array}$ & & & & & & & & & & & \\
\hline $\begin{array}{c}\text { rosR } \\
\text { RL1379 }\end{array}$ & & & & & & & & & & & \\
\hline $\begin{array}{c}\text { fnrN } \\
(\mathrm{RL2818)}\end{array}$ & 2 & 2 & & & & & 2 & & & 2 & \\
\hline $\begin{array}{c}\text { cheA } \\
\text { RL0688 }\end{array}$ & & & & & & & & & & & \\
\hline $\begin{array}{c}\text { fabF1 } \\
\text { (RL2814) }\end{array}$ & & & & & & & & & & & \\
\hline $\begin{array}{c}\text { pssD } \\
\text { (RL3654) }\end{array}$ & & & & & & & & & & & \\
\hline $\begin{array}{c}\text { plyA } \\
\text { (RL3659) }\end{array}$ & & & & & & & & & & & \\
\hline pRL110330 & & & & & & & & & & & \\
\hline RL2862 & & & & & & & & & & & \\
\hline $\begin{array}{c}\text { dctM } \\
\text { (pRL120498) }\end{array}$ & & & & & & & & & & & \\
\hline RL1858 & & & & & & & & & & & \\
\hline $\begin{array}{c}\text { qat6X } \\
\text { (pRL100081) }\end{array}$ & 2 & 2 & & & & & & & & & \\
\hline $\begin{array}{c}\text { haat } \\
(p R L 100131)\end{array}$ & & & & & & & & & & & \\
\hline $\begin{array}{c}\text { attL } \\
\text { (pRL100135) }\end{array}$ & & & & & & & & & & & \\
\hline pRL100104 & 2 & 2 & & & & & 2 & & & & \\
\hline pRL100107 & & & & & & & & & & & \\
\hline pRL100120 & $2,139 b p$ & $2,139 \mathrm{bp}$ & $426 \mathrm{bp}$ & & & $426 \mathrm{bp}$ & $2,139 b p$ & & & & $426 \mathrm{bp}$ \\
\hline
\end{tabular}

G1 03 (GPS1 03), G2 15 (GPS2 15), L1 35 (LPS1 35), H1 09 (HPS1 09), H2 12 (HPS2 12), V1 17 (VPS1 17), V2_25 (VPS2_25), Em (Ensifer méliloti 1021), Re (Rhizobium etli CFN42), Rlv UPM791 (R. Teguminosarum bv. viciae UPM791) and RIv3841 (R. leguminosarum bv. viciae 3841).

Figure 3.S4 and Supplementary Figure 3.S5). Genes dctM and RL1858 were not present in VPS2_25 genome, and it is worth noting that a second copy of fnrN (fixK) was present in the symbiotic plasmid of strains GPS1_03, GPS2_15 and VPS2_25.

Other differentially host-selected mesocosm genes (qat6X, attL, pRL100104, pRL100107 and pRL100131), that were located in the R. leguminosarum bv. viciae 3841 symbiotic plasmid, were absent in fava bean mesocosm Pool-Seqs. These genes generated similar phylogenetic relationships among the remaining mesocosm-selected 
strains, defining two groups: group I (LPS1_35, VPS1_17 and R. leguminosarum bv. viciae 3841) and group II (GPS1_03, GPS2_15 and VPS2_25) (Supplementary Figure 3.S6).

Gene pRL100120 was described above as a differential gene among mesocosm subpopulations; this gene is present in the reference genome $R$. leguminosarum bv. viciae 3841, it is $426 \mathrm{bp}$ in length, and was also found in LPS1_35 and VPS1_17. However, for GPS1_03, GPS2_15 and VPS2_25 it had 2,139 bp, and in the BlastN output two domains were defined, an acetate/3-ketoacid CoA transferase in C-terminal and a Phasin_2 domain in N-terminal. This Phasin_2 domain was the gene pRL100120.

\subsubsection{Exclusive genes in plant host mesocosm-selected strains}

A basic comparative genomic analysis was performed in order to find exclusive host mesocosm-selected genes for each subpopulation, that were not present in the reference genome $R$. leguminosarum bv. viciae 3841 nor in the other host mesocosmselected genomes. Different values were obtained for each host mesocosm-selected genomes; a total of 45 genes were exclusively present in pea (GPS1_03 and GPS2_15) , 303 genes in lentil (LPS1_35), 42 in vetch (VPS1_17 and VPS2_25), and none in fava bean (HPS1_09 and HPS2_12) host mesocosm-selected strains (Supplementary Table S9). None of the putative functions assigned to those genes by BLAST similarity would appear to be directly related to preference by a specific host. For instance, many of the genes exclusively present in both pea mesocosm-selected strains encoded different enzymes, transposases, transcriptional regulators, transport system components or hypothetical proteins. 


\subsection{DISCUSSION}

\subsubsection{Conservation of sequences from the Rhizobium leguminosarum bv. viciae 3841 reference genome in mesocosm plant-selected rhizobial populations}

The global differences observed in recruitment profiles (Figure 3.1 to 3.4 and Supplementary Figure 3.S1 to 3.S3) reflected the conservation / absence / overrepresentation of genes from the reference genome in plant-selected populations (Figures 3.5 to 3.7 ). Globally, $73 \%$ of $R$. leguminosarum bv. viciae CDSs (5,312 of $7,269)$ were conserved in all host-selected Pool-Seqs, which defines a genetic core that is preserved in the $\mathrm{P} 1$ soil $R$. leguminosarum bv. viciae population. This core value is similar in size to genetic cores found for other related species, such as Ensifer medicae (5,036 core genes for 12 genomes), E. fredii $(5,979$ core genes for 2 genomes) or E. meliloti (4,680 core genes for 32 genomes) (Sugawara et al., 2013). The actual numbers of genes defining the genetic core will be dependent on the genetic variability of the species considered (or whether one or more species are considered), on the number of genomes analysed, and on the percentage identity threshold considered for comparisons. For instance, Galardini et al. (2011) found that the core genome for $E$. meliloti was 5,124 genes, however the number of genomes used was only three. On the contrary, a recent paper published by Kumar et al. (2015), where they studied the $R$. leguminosarum population isolated from Vicia cracca and Trifolium repens showed that, at the nucleotide level and with a threshold of $90 \%$ identity, only 305 core genes where present in the 72 genomes analysed.

The mesocosm experiment showed differences in certain regions and genes among host-selected subpopulations; for instance, pea plants preferred microsymbionts containing sequences that are localized in pRL7 in the reference genome (Figure 3.4, panel A and B). Some of these significantly enriched regions were further enriched in pea mesocosm Pool-Seqs over the initial host subpopulation (overrepresented peak $\mathrm{pRL} 7_{\mathrm{b}}$, Table 3.2). Other overrepresented peaks were exclusively detected in pea mesocosm subpopulations, such as $\mathrm{Ch}_{\mathrm{g}}$ in the chromosome (Figure 3.1). In this region two genes (RL4249-50) were annotated as part of a CUT1 type ABC transport system. RL4249 to RL4252 were previously described to be strongly repressed in free-living cultures by growth on either succinate or pyruvate rather than on glucose; however, single mutants in RL4249 were able to grow on glucose as the sole carbon source, which indicates the redundancy of this CUT1 type $A B C$ transporters (36 in the genome of $R$. leguminosarum bv. viciae; (Karunakaran et al., 2009). 
Large differences in the initial region of the pRL10 plasmid were detected among mesocosm host-selected subpopulation (Figure 3.2). This corresponds to a low coverage (region 7; pRL100011-pRL100138) that was nonetheless conserved in the initial host-selected subpopulations (Jorrín and Imperial, 2015b). However, this changed after the mesocosm experiment: The whole low coverage region (128 genes) was maintained in lentil and fava bean Pool-seqs, while it was not in vetch and pea Pool-Seqs. Only 28 (subregion pRL10 1 ) or $63(\mathrm{pRL10})$ genes were maintained in pea and vetch Pool-seqs, respectively. Within the 28-gene pRL10 $1_{1}$ subregion, pea subpopulations presented new, overrepresented peaks: pRL10 $0_{a}$ and $p R L 10_{d}$. In $\mathrm{pRL} 10_{\mathrm{a}}$ a qat (quaternary ammonium transporter) of the $\mathrm{ABC}$ family (qat6VW) was localized; this $A B C$ system has been implicated in the transport of histidine/proline/ proline-betaine/ glycine-betaine/choline that are used as osmoprotectants in Ensifer spp. (Boncompagni et al., 1999, Boscari et al., 2002, Dupont et al., 2004, Alloing et al., 2006). In R. leguminosarum bv. viciae 3841 previous studies showed that the qat6 cluster is strongly induced by hyperosmotic stress $(100 \mathrm{mM} \mathrm{NaCl})$, however the solute transported has not been identified (Fox et al., 2008).

Two other overrepresented peaks were found exclusively in pea mesocosm, hostselected populations: pRL10 fixO copy (pRL10d, Figure 3.2) and pRL9 fixIH copies (pRL9 ${ }_{a}$, Figure 3.S3), which suggests that genes from the fixNOQPGHIS cluster (present in two copies in the reference genome) enriched in pea mesocosm subpopulations were more similar to either one or the other ( $p R L 9$ or $p R L 10$ ) of the available copies in the $R$. leguminosarum bv. viciae 3841 reference genome. These overrepresentations were accompanied by a significant reduction in pRL10 fixHIS genes coverage (Supplementary Table S4). Genome sequence analysis of a representative strain from each of the pea mesocosm experiments (GPS1_03 and GPS2_15; see Figure 3.23) showed that both strains harbour two complete copies of the fixNOQPGHIS cluster genomes (data not shown), which suggests that the observed differences in coverage are probably due to low identity between reference and selected fix clusters rather than to selection of a single copy.

Another striking difference in relative abundance was the depletion in both fava bean mesocosm subpopulations of the phnA gene (Supplementary Table S4), annotated as a poly-ß-hydroxybutyrate synthase protein. PHB is accumulated in undifferentiated rhizobia, and it is suggested to support rhizobia in saprophytic conditions, because it enhances bacterial survival under starvation (Lopez et al., 1995, Denison and Kiers, 2004).

Vetch Pool-Seqs presented the smallest core of conserved genes. This was a

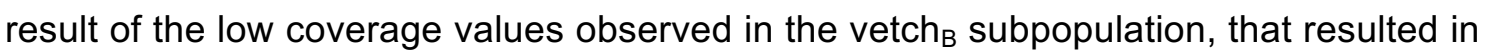


turn in the depletion of over 1,500 genes in this subpopulation during the mesocosm experiment (Figure 3.9). These results suggest that this subpopulation was different from the reference genome and from the other two vetch-selected Pool-Seqs (initial vetch and vetch A). .

Overall, more genes from the reference genome were depleted than enriched during the mesocosm experiment, which could be taken to indicate a reduction in the genome of selected subpopulations. This would no be unheard of in the rhizobia, since Candidatus Liberibacter sp., a pathogen that causes Huanglongbing disease in Citrus species and that belongs to the order Rhizobiales, is unable to grow in regular culture media because they lack certain metabolic pathways due to an important genome erosion in this pathogen (Tyler et al., 2009). An incipient genome erosion in mesocosm experiments is, however, unlikely, since no clear significant genome reduction could be observed in the genomes of mesocosm-selected strains (Table 3.7). Another possible explanation for gene depletion would be that of gene differentiation in comparison to the reference $R$. leguminosarum bv. viciae 3841 genome. As previously discussed, only those reads with at least $85 \%$ identity were recruited and, therefore, analysed. While the $77.2-84.2 \%$ of the Pool-Seq reads from the initial host-selected subpopulations were recruited to the reference genome (Table 2.13), a reduction in the percentage of recruited reads was observed for mesocosm host-selected subpopulations (pea $66.64 \%$, pea $83.87 \%$ lentil $_{A} 80.92 \%$, lentil $_{B} 81.55 \%$, fava bean $_{A}$ $77.27 \%$, fava bean ${ }_{B} 76.23 \%$, vetch $79.83 \%$ and vetch $64.33 \%$ of aligned reads). Although outside the temporal and material scope of this Thesis work, it would be very interesting to investigate further changes in genome composition of host-selected subpopulations by further iterating the mesocosm selection steps, as well as by increasing the number of replicates in order to separate specific plant-host selection from possible stochastic, bottleneck-related selection forces.

\subsubsection{Genome-wide sequence polymorphisms in mesocosm Pool-Seq datasets}

The level of differentiation of plant-host selected subpopulations was globally analysed by recording percentages and global averages of positions with significant $F_{\mathrm{ST}}$ value for each Pool-Seq pairwise comparison. The global $F_{\mathrm{ST}}$ average showed no significant values (above the 0.25 threshold) for any pairwise comparison, probably due to the dilution of differences. Nevertheless, both analyses showed similar trends of variation. $V$ etch ${ }_{B}$ and pea $_{B}$ were the most dissimilar subpopulations, which impeded clusterization of pea and vetch mesocosm Pool-Seqs. A high diversity within pea and vetch microsymbionts was previously demonstrated by Mutch and Young (2004), who 
found that the number of different genotypes that nodulate pea (nineteen) and vetch (nineteen) was higher than the different genotypes present in fava bean (eleven). Similar observations were reported by Laguerre et al. (2003), who found six different genotypes in fava bean nodules, as compared to eleven for pea nodules.

At the opposite side of the spectrum, a high conservation was observed within lentil-selected subpopulations. This does not necessarily mean that lentil was nodulated by a single genotype, but rather that the same genotypes, and in similar proportions as those present in the soil, nodulate lentil, suggesting that lentil is a nonselective host. In fact, when rpoB and nodC genotypes were studied for each component strain of the lentil mesocosm Pool-Seqs, a high number of different genotypes were found (Figure 3.17). This large genotypic diversity in lentil nodules had previously gone mostly unnoticed, although it can be deduced from published reports. Hynes and Oconnell (1990) showed that lentil symbionts present the same diversity level as pea, but their lentils were poorly nodulated, and the low number of isolates that they obtained (20 and 60 for lentil and pea respectively) could have resulted in a distortion of results. Laguerre et al. (1992), showed that rhizobial genotypes were more diverse in lentil nodules than in pea when three different markers (plasmid profiles, chromosomal hybridization patterns and nod gene probes) were used. A further study performed in the North of Algeria found no significant differences at diversity level between pea and lentil populations, but this could be due to a reported low diversity of $R$. leguminosarum in soil (Riah et al., 2014). Finally, three new linages of lentil microsymbionts were found in Bangladesh, with nodulation gene sequences showing high similarity to $R$. leguminosarum bv. viciae (Rashid et al., 2013) that were later defined as three new species (Rhizobium lentis, $R$. binae and $R$. bangladeshense (Rashid et al., 2015). Despite these data documenting a high diversity among lentil microsymbionts, as discussed in Chapter 2 (section 5.3.2), no previous evidence had been found for lentil as a non-selective host, partly because the native soil Rhizobium leguminosarum population had not been analysed in any of these studies.

The global analysis of polymorphisms in mesocosm plant-host selected subpopulations confirmed previous observations with initial plant-host selected subpopulations, where the pea initial subpopulation was different from soil population and from lentil, fava bean and vetch initial subpopulations (Chapter 1 and Chapter 2 , Figure 3.10).

Next, the distribution of polymorphisms was studied at the CDS level, and $F_{\mathrm{ST}}-\mathrm{CDS}$ values were calculated and compared. A total of 1,243 CDSs were non-informative due to the lack of recruited reads in at least one of the subpopulations investigated, which made the comparison analysis impossible. Globally, only 99 CDSs among those 
present $(B P K M>10)$ showed no significant differentiation among all subpopulations $\left(F_{\mathrm{ST}}<0.1\right)$, which probably indicates that these genes allow a limited number of polymorphisms and are probably essential for $R$. leguminosarum. In accordance with this hypothesis, the presence of most of the tRNA genes (36 out of 52) and the rRNAs is remarkable. However, it is also noteworthy that differences among each hostselected subpopulation were observed for one copy of $16 S$ and $23 S$ in fava bean $_{B}$ and vetch $_{B}$ subpopulations, which suggests that not all the rRNA copies are identical. Divergences among rRNA copies present in the same genome were previously reported for Bacillus spp., Clostridium spp. or Salmonella spp. (Klappenbach et al., 2001, Větrovský and Baldrian, 2013). Furthermore, when the complete rRNA cluster, rather than the separate $16 \mathrm{~S}$ and $23 \mathrm{~S}$ rRNA genes, was studied, only lentil-selected subpopulations clustered together (Figure 3.12 panels $A, B$ and $C$ for copies 1,2 and 3 respectively). This divergence in results between $16 \mathrm{~S} / 23 \mathrm{~S}$ and the complete rRNA clusters can be attributed to differences found in the intergenic spacer (IGS) between rRNA genes (that were included for rRNA analysis); this is in line with the use of restriction enzyme IGS patterns as genotype markers in $R$. leguminosarum studies (Louvrier et al., 1995, Laguerre et al., 1996, Laguerre et al., 2003, Mutch and Young, 2004).

Large differences in the number of host-selected CDSs (intra-population $F_{\mathrm{ST}}<0.1$ ) were found among host-selected subpopulations. Within lentil-selected subpopulations $85.02 \%$ of the CDSs analysed $(5,049$ out of 5,938$)$ presented a $F_{\mathrm{ST}}<0.1$; the remaining CDSs presented highly significant differences $\left(F_{\mathrm{ST}}>0.25\right)$. In Chapter 2 of this Thesis we showed that the initial lentil subpopulation accurately reflected the diversity present in soil, since only $0.082 \%$ of the nucleotide positions were SD-SNPs (Chapter 2, Table 2.5). After mesocosm lentil selection this percentage dropped to $0.0417 \%$ (Table 3.4). The same observation was done for the remaining initial host-selected subpopulation. This drop was due to the absence of reads in at least one of the mesocosm Pool-Seqs. Technically, this makes the comparison inherent to the Popoolation2 algorithm impossible. Despite this limitation, the fact was that lentil subpopulations (initial and mesocosm Pool-Seqs) were reasonably conserved among themselves, and also with the soil population, as only 49 (lentil $\left._{A}\right), 20$ (lentil $_{B}$ ) and 4 (initial lentil) CDSs out of 5,938 showed a $F_{\mathrm{ST}}$ value greater than 0.25 in comparison with soil population.

Remarkably, the different pea host-selected subpopulations presented important differences at the level of symbiotic genes. The initial pea subpopulation showed significant differences in comparison with pea $_{A}$ and pea $_{B}$ Pool-Seqs (Figure 3.13). Indeed, when comparing pea $_{\mathrm{A}}$ and pea $_{\mathrm{B}}$ subpopulations, the average $F_{\mathrm{ST}}$ values for most of the genes in the symbiotic cluster was above 0.1 (with the exception of nif-fix 
that was 0.09). These results indicated that pea plants were nodulated by a broad range of symbiotic genotypes. This had been already suggested by Mutch and Young (2004), who hypothesised that the higher diversity found in pea nodules could be due to a "relatively broad symbiotic specificity", and therefore the reason of the successful spread of pea cultivation. These results contrast with the 1,143 host-selected CDSs that were found among pea subpopulations (genes for chemotaxis, motility, ABC transporters, EPS synthesis and fatty-acid modification), and altogether indicate that pea plants do not select at the level of nodule formation and operation, but rather at the level of rhizospheric life, where certain Rhizobium leguminosarum rhizospheric genotypes would have an advantage in pea nodulation, irrespective of the symbiotic genotype that was carried along.

Regarding fava bean, despite the low number of host-selected CDSs, fava bean subpopulations did not present any CDS with a $F_{\mathrm{ST}}$ above 0.25. Among these fava bean-selected CDSs were most of the symbiosis-related genes. However, this was not necessarily the case for the whole symbiotic plasmid, for which fava bean $\mathrm{A}_{\mathrm{A}}$ and fava bean $_{B}$ presented a $F_{S T}=0.12$. It appears that fava bean plants were very restrictive regarding the symbiotic genotypes that would able to form nodules. Evidence of this had been previously presented. By studying RLFP types for the nodDF intergenic region, Mutch and Young (2004) showed that fava bean plants were more restrictive than pea plants, and were predominantly nodulated by their nodDF-2 type. Similarly, Laguerre et al. (2003) observed that fava bean plants were mostly nodulated by nodDF type g. Both genotypes appear to be the same and seemed to be the most abundant in soils from the United Kingdom (Mutch and Young, 2004) and France (Laguerre et al., 2003). However, in another study on V. faba microsymbionts in Jordan (geographic area of fava bean domestication (Bond, 1976)), this nodDF-2/g type was present in nodules (Mutch et al., 2003). Taken together with our genome-wide results, the available evidence suggests that fava bean plants select for a restricted range of symbiotic genotypes that are not always localized in the same symbiotic plasmid, or within the same chromosomal background.

No conclusive results were obtained for vetch subpopulations. In Chapter 2 we saw that initial vetch subpopulation was slightly (but not significantly) different from the soil population, which that vetch held an intermediate position between pea-selected and lentil/fava bean-selected subpopulations. Results presented in this Chapter show that

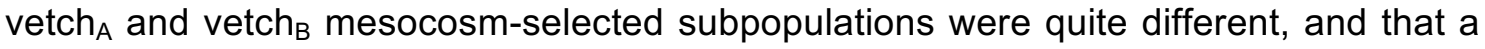
low number of CDSs were selected (87), as well as with the initial vetch-selected subpopulation (72). Initial vetch and vetch $_{A}$ were quite similar (5,523 CDSs with $\left.F_{S T}<0.1\right)$, which reminds of the situation encountered with lentil subpopulations. 
However, between initial vetch and vetch ${ }_{B}$ only 111 CDSs had and $F_{S T}<0.1$, which would in turn suggest a pea-like situation. Most of the 87 vetch-selected CDSs had a BPKM below 10 for vetch ${ }_{B}$, with the exception of: $c p s A$, atpC, RL0465A, RL2501 (LysR-like transcriptional regulator), pRL100095, and tRNA-Ala2, tRNA-GIn2 and tRNA-Trp. In addition, a few mesocosm vetch-selected CDSs were conserved $(B P K M>10)$ in vetch ${ }_{B}:$ RL0297, RL2304, RL2451 and genes located in the region pRL100100-pRL100133, among them certain components of the HAAT ABC transporter (ATP-binding protein and the permease). However, analysis of the complete HAAT cluster ( $p R L 100127-p R L 100131)$ showed highly significantly differences between vetch $_{A}$ and vetch $_{B}$ (Figure 3.15).

Regarding inter-host comparisons, different regions were analysed in detail (section 6.2.1.8.4, Figures 3.15 and 3.16). Only one gene, pRL100120, presented a $F_{\mathrm{ST}}<0.1$ within host-selected subpopulations, and it allowed differentiation of vetch and pea from fava bean, and these from lentil (Figure 3.16, panel F). pRL100120 is located in the low coverage region 7 , and although in the combined Pool-Seqs the coverage value was above $10 \mathrm{BPKM}$, in some of them the coverage was low: 38.9 (lentil ${ }_{B}$ ), 11.7 (fava bean ${ }_{A}$ ), 34.7 (fava bean ${ }_{B}$ ), 15.2 ( (vetch $_{A}$ ), 27.3 (initial host average) and 13.8 (soil) BPKM. This is a small, $426 \mathrm{bp}$ gene annotated as a hypothetical protein, and shows a conserved Phasin_2 domain. Phasins are granule-associated proteins found covering PHA (polyhydroxyalkanoate) storage granules in bacteria; a layer of phasins stabilises the granules of PHA and prevents coalescence of separated granules in the cytoplasm (de Almeida et al., 2007). Phasins were expressed during PHB (poly- $ß-$ hydroxybutyrate, the most common PHA) synthesis in Bradyrhizobium japonicum (Yoshida et al., 2013). Moreover, the expression of pRL100120 is 9-fold higher in bacteroids than in free-living bacteria for $R$. leguminosarum bv. viciae 3841 (Karunakaran et al., 2009). When the presence of pRL100120 homologs was investigated in the genomes of mesocosm-selected strains, we found that fava beanselected strains did not have any pRL100120 homologue, while VPS1_17 and LPS1_35 had exactly the same gene as R. leguminosarum bv. viciae 3841 (Table 3.11). However, homologues found in GPS1_03, GPS2_15 and VPS2_25 were longer $(2,139 \mathrm{bp})$, and the Phasin_2 domain was located in an N-terminal position, while an acetate/3-ketoacid CoA transferase was located at the $\mathrm{C}$ terminus. When the Phasin_2 domain of mesocosm-selected strains was compared, only two nucleotides were different between the two groups, and they translated into different amino acids (Val/Val for VPS1_17 and LPS1_35; and Ala/Phe for GPS1_03, GPS2_15 and VPS2_25). PHB synthesis has been reported to compete with nitrogen fixation (Anderson and Dawes, 1990); for instance in indeterminate nodules, PHB 
accumulation decreases during the bacteroid development (Vasse et al., 1990), and $R$. leguminosarum mutants that accumulate PHB in bacteroids showed a Fix phenotype despite of the presence of nitrogenase protein (Lodwig et al., 2003).

Another gene for which inter-host differences were found was pRL100104 (Figure 3.16, panel D). This gene allowed differentiation of lentil, fava bean and vetch mesocosm subpopulations. However, low coverage values were found in fava bean Pool-Seqs (11.4 and 35.5 for fava bean $\mathrm{B}_{\mathrm{A}}$ and fava bean $_{B}$, respectively). These results correlated with the absence of pRL100104 homologues in fava bean mesocosmselected strains (Table 3.11). Two copies of pRL100104 homologues were found for GPS1_03, GPS2_15 and VPS2_25, and both copies were different from those present in LPS1_35 and VPS1_17 (Supplementary Figure 3.S6, panel D). Gene pRL100104 is annotated as phaE (Poly(R)-hydroxyalkanoic acid synthase) and it could play a role in the synthesis of PHA. pRL100104 is also up-regulated in mature bacteroids (Karunakaran et al., 2009), and the distribution among the isolates used in the Kumar et al. (2015) study suggested that it may be transferred together with the att $\gamma$ hydroxybutyrate degradation cluster (attJKLM, pRL100133-pRL100136). In view of this observation, we also studied the att cluster. It allowed differentiation of pea, lentil and fava bean mesocosm subpopulations (Figure 3.15, panel C). However, no homologues were detected in the genomes of fava bean mesocosm-selected strains (Table 3.11); therefore two different groups were defined: LPS1_35 and VPS1_17; and GPS1_03, GPS2_15 and VPS2_25 (Supplementary Figure 3.S6, panel C). The att cluster allows the conversion from $\gamma$-butyrolactone to succinate via $\gamma$-hydroxybutyrate for use as $\mathrm{C}$ source (Carlier et al., 2004). R. leguminosarum bv. viciae 3841 attL mutants were unable to grow on $\gamma$-hydroxybutyrate (Kumar et al., 2015).

Similarly, qat6 and haat $A B C$ transporters (Figure 3.15, panels $A$ and $B$ respectively) allowed for a similar Pool-Seq clusterization pattern, and no homologues were found for any of them in fava bean mesocosm-selected strains (Table 3.11). 


\subsubsection{Genomics of mesocosm-selected strains}

The $r p o B$ and nod $C$ diversity of mesocosm Pool-Seq subpopulations was analysed and a representative from the most abundant genotype was selected for individual genome sequencing, with the exception of lentil subpopulations, where only one strain was selected from the most abundant, exclusive genotype found (LPS1_35). The genome of this strain was able to recruit over $80 \%$ of the total reads of lentil mesocosm Pool-Seqs, and $2.7-3.8 \%$ of the nucleotide positions of the LPS1_35 genome were polymorphic in these Pool-Seqs (Table 3.9). This was consistent with the fact that lentil populations were always found to present the highest diversity values (Hynes and Oconnell, 1990, Laguerre et al., 1992, Riah et al., 2014). On the other hand, only one genotype was found for pea $_{B}$ and vetch $_{B}$ subpopulations, and more than $98 \%$ of its Pool-Seqs were recruited to the genome of the mesocosm-selected strain for each subpopulation. This paralleled the lowest SNPs values found in this study (Table 3.9).

One of the most surprising results was the inability of GPS1_03, GPS2_15 and VSP2_25 to form nodules in fava bean plants (Table 3.6). The existence of strains unable to nodulate fava bean had been previously observed by Mutch and Young (2004), where only $16 R$. leguminosarum strains out of 70 isolated from other plant species (Vicia cracca, V. hirsuta, V. sativa, Lathyrus aphaca, L. pratensis and Pisum sativum) were able to form nodules in fava bean. Our three mesocosm-selected strains unable to nodulate fava bean presented the same symbiotic cluster organization (Figure 3.25), as well as the same genotype for nif, rhi, nod and nif-fix clusters (Figure 3.19-3.22). However differences were observed in the fix cluster, mostly due to the presence of more than one contig in pea mesocosm-selected strains, as well as to the presence of a different transcriptional regulator downstream of fixS (FNR/CRP-like in GPS1_03, and $\sigma-54$ in GPS2_25 and VPS2_25, Figure 3.23). Although this cluster organization correlated with the inability to form nodules in fava bean, it must be borne in mind that $R$. leguminosarum bv. viciae UPM791, a strain that shares this symbiotic cluster organization (Figure 3.25) is able to nodulate effectively fava bean (López et al., 1983)

Regarding fava bean selected strains, despite the genomic and symbiotic region conservation between HPS1_09 and HPS2_12 (Figure 3.18 and Figure 3.24), none of the symbiotic subregions was found to cluster together (Figure 3.19 to 3.23). These results were not congruent with those obtained in the mesocosm Pool-Seq analysis, where fava bean $n_{A}$ and fava bean ${ }_{B}$ shared their same symbiotic genotypes (although neither the symbiotic plasmid nor the chromosomal markers). As it was already suggested in the results section of this chapter, it appears that HPS1_09 does not 


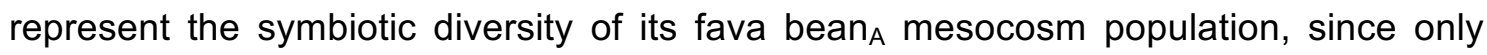
$76.89 \%$ of the fava bean A $_{\text {reads }}$ were recruited by HPS1_09, and $2.965 \%$ of the genome nucleotide positions were polymorphic. Therefore, it would appear that HPS1_09 was chosen in error, and that other strain(s) should be chosen instead to

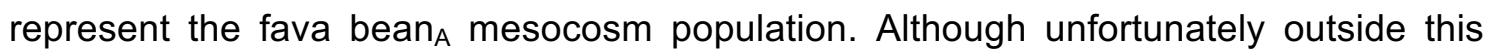
work, genome sequencing and characterization of two further fava bean strains (HPS1_03 and HPS1_22) is currently underway. 


\subsection{SUPPLEMENTARY MATERIAL}

Supplementary Table 3.S1. Non significant CDSs present in all Pool-Seqs (1/2)

\begin{tabular}{|c|c|c|c|}
\hline Product & Gene & & \\
\hline 5S rRNA & RLr07 & RLr08 & RLr09 \\
\hline tRNA-Ala & tRNA-Ala_1 & tRNA-Ala_3 & tRNA-Ala_4 \\
\hline tRNA-Arg & tRNA-Arg_1 & tRNA-Arg_2 & tRNA-Arg_3 \\
\hline tRNA-Arg & tRNA-Arg_4 & & \\
\hline tRNA-Asn & tRNA-Asn_1 & & \\
\hline tRNA-Asp & tRNA-Asp_1 & tRNA-Asp_2 & \\
\hline tRNA-Cys & tRNA-Cys_1 & & \\
\hline tRNA-Glu & tRNA-Glu_2 & & \\
\hline tRNA-Gly & tRNA-Gly_1 & tRNA-Gly_2 & tRNA-Gly_3 \\
\hline tRNA-His & tRNA-His_1 & & \\
\hline tRNA-Ile & tRNA-Ile_1 & tRNA-Ile_2 & tRNA-IIe_3 \\
\hline tRNA-Leu & tRNA-Leu_1 & tRNA-Leu_3 & tRNA-Leu_4 \\
\hline tRNA-Leu & tRNA-Leu_5 & & \\
\hline tRNA-Lys & tRNA-Lys_2 & & \\
\hline tRNA-Met & tRNA-Met_2 & tRNA-Met_4 & \\
\hline tRNA-Phe & tRNA-Phe_1 & & \\
\hline tRNA-Pro & tRNA-Pro_1 & tRNA-Pro_2 & \\
\hline tRNA-Ser & tRNA-Ser_1 & tRNA-Ser_2 & tRNA-Ser_5 \\
\hline tRNA-Thr & tRNA-Thr_1 & tRNA-Thr_2 & \\
\hline tRNA-Tyr & tRNA-Tyr_1 & & \\
\hline tRNA-Val & tRNA-Val_2 & & \\
\hline putative GroEL 60 kDa chaperonin & groL & & \\
\hline aldo/keto reductase & RL0303 & & \\
\hline CoA reductase & RL0304 & & \\
\hline cytochrome c & RL2498 & RL3855 & \\
\hline epoxide hydrolase & RL1848 & & \\
\hline glyoxalase/dioxygenase & RL2846 & & \\
\hline putative cytosine deaminase & pRL110609 & & \\
\hline putative enoyl-CoA hydratase & pRL120176 & & \\
\hline putative flavonol synthase/dioxygenase & pRL110611 & & \\
\hline $\begin{array}{l}\text { putative glycerophosphoryl diester } \\
\text { phosphodiesterase }\end{array}$ & pRL90114 & & \\
\hline putative hydrolase & pRL100066 & & \\
\hline $\begin{array}{l}\text { ribitol dehydrogenase, determined } \\
\text { experimentally to be required for growth on } \\
\text { ribitol }\end{array}$ & pRL90116 & & \\
\hline $\begin{array}{l}\text { PTS-dependent dihydroxyacetone } \\
\text { kinase,ADP-binding subunit }\end{array}$ & dhaL 1 & & \\
\hline putative small heat shock protein & pRL120342 & & \\
\hline $\begin{array}{l}\text { two-component response regulator } \\
\text { transcriptional regulatory protein }\end{array}$ & RL2277 & RL3599 & \\
\hline AraC family transcriptional regulator & RL0302 & & \\
\hline $\begin{array}{l}\text { putative FNR/CRP family transcriptional } \\
\text { regulator }\end{array}$ & pRL90025 & & \\
\hline cell envelope protein & RL3058 & & \\
\hline efflux RND transporter protein & RL3602 & & \\
\hline $\begin{array}{l}\text { putative ATP-binding component of ABC } \\
\text { transporter }\end{array}$ & pRL100070 & pRL90110 & \\
\hline putative MFS family transporter & pRL120127 & & \\
\hline $\begin{array}{l}\text { putative permease component of } A B C \\
\text { transporter }\end{array}$ & pRL90260 & pRL90112 & pRL90113 \\
\hline
\end{tabular}


Supplementary Table 3.S1. Non significant CDSs present in all Pool-Seqs (2/2)

\begin{tabular}{|c|c|c|c|}
\hline Product & Gene & & \\
\hline $\begin{array}{l}\text { putative transmembrane CrtK/TspO-family } \\
\text { sensor protein }\end{array}$ & pRL90314 & & \\
\hline $\begin{array}{l}\text { putative transmembrane protein } \\
\text { putative transmembrane tryptophan-rich }\end{array}$ & pRL90006 & & \\
\hline $\begin{array}{l}\text { protein } \\
\text { transmembrane component of } A B C\end{array}$ & pRL120571 & & \\
\hline transporter & RL1746 & & \\
\hline DNA-damage-inducible protein & RL2523 & & \\
\hline FlaR DNA topology modulation protein & RL2250 & & \\
\hline putative plasmid stability protein StbB & pRL120076 & & \\
\hline site-specific DNA recombinase & RL3956 & & \\
\hline conserved hypothetical protein & pRL100099 & pRL120483 & pRL90024 \\
\hline hypothetical protein & RL1198 & RL2482 & RL2867 \\
\hline hypothetical protein & RL3012 & RL3442 & RL3988 \\
\hline hypothetical protein & RL4094 & pRL120114 & \\
\hline no description & pRL100100 & pRL70043 & \\
\hline pseudogen & RL4510 & & \\
\hline
\end{tabular}




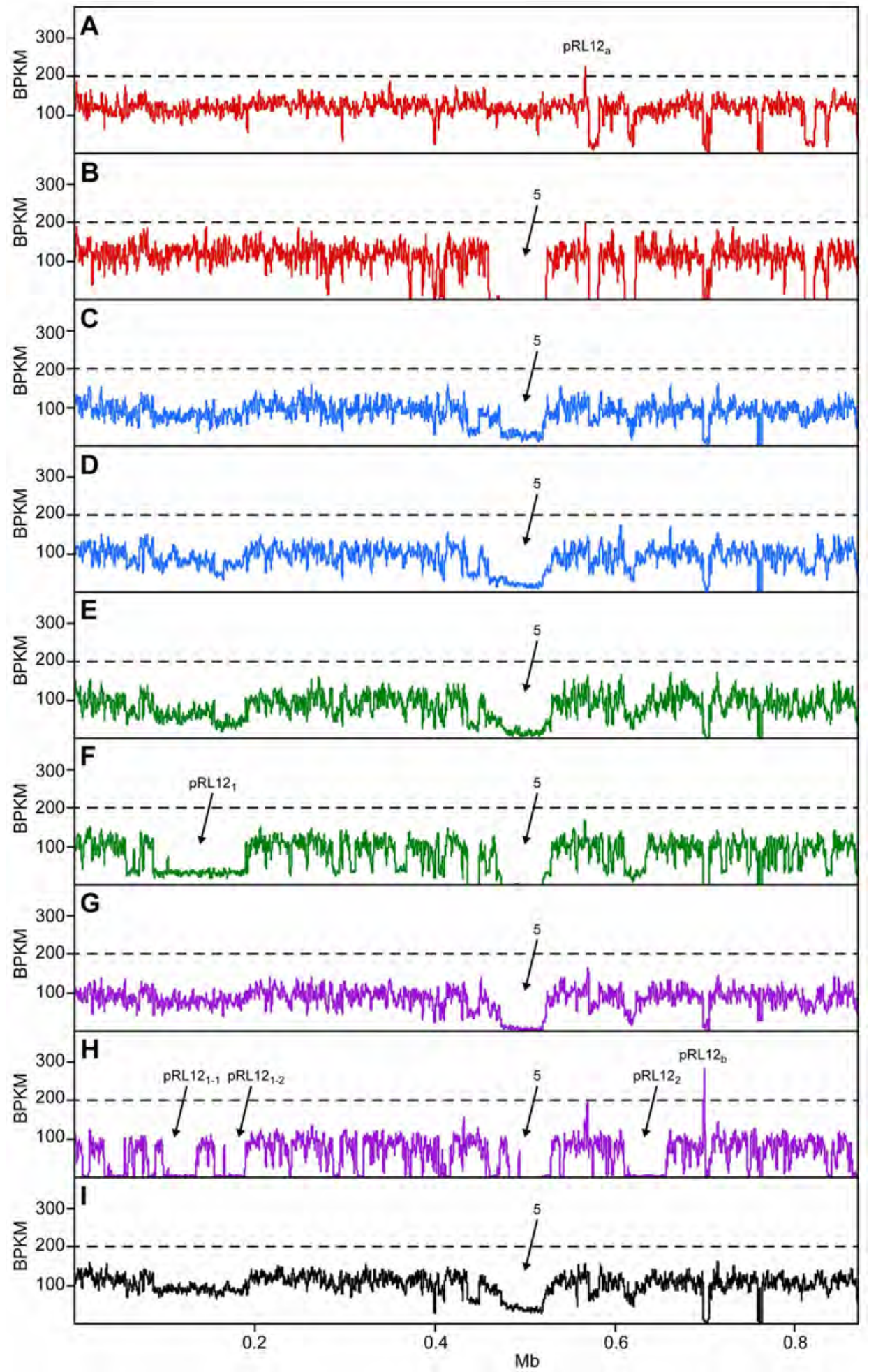

Supplementary Figure 3.S1. Conservation of the plasmid pRL12 of $R$. leguminosarum bv. viciae 3841 reference genome in the mesocosm plant-selected subpopulations. Coverage (BPKM) of genome regions along $p R L 12$ by Bowtie2 recruited reads from pea $A(A)$, pea $_{B}$ $(B)$, lentil $(C)$, lentil $(D)$, fava bean $A_{A}(E)$, fava bean $(F), \operatorname{vetch}_{A}(G)$, vetch $B(H)$ and host $(I)$. Major under- and over-represented regions in the mesocosm plant-selected datasets are indicated numerically or as pRL12 $1-2$, and as $\mathrm{pRL} 12_{\mathrm{a}-\mathrm{b}}$, respectively. 


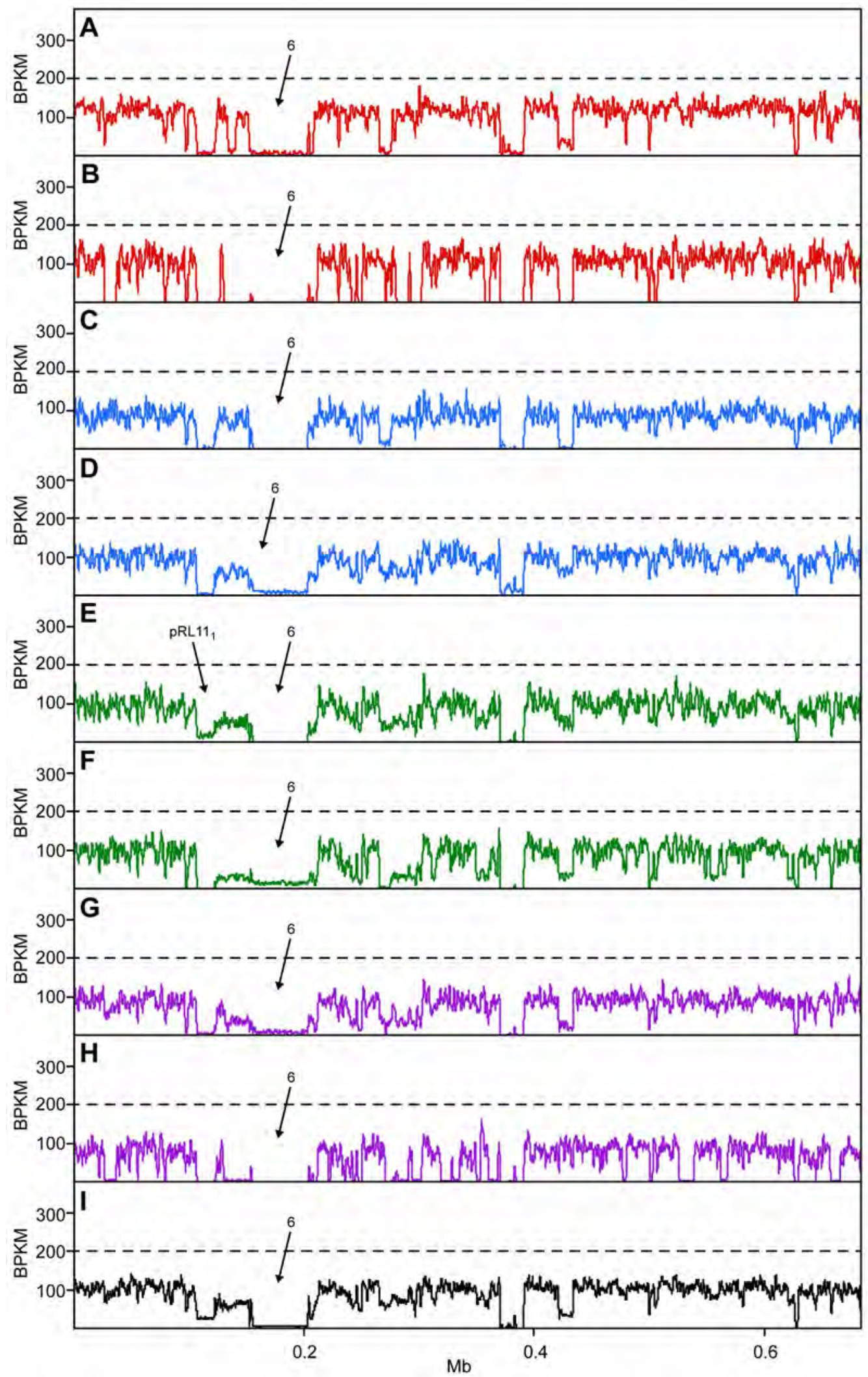

Supplementary Figure 3.S2. Conservation of the plasmid pRL11 of $R$. leguminosarum bv. viciae 3841 reference genome in the mesocosm plant-selected subpopulations. Coverage (BPKM) of genome regions along pRL11 by Bowtie2 recruited reads from $\mathrm{pea}_{A}(A), \mathrm{pea}_{B}$ $(B)$, lentil $A(C)$, lentil $B(D)$, fava bean $A_{A}(E)$, fava bean $B(F), \operatorname{vetch}_{A}(G)$, vetch $B(H)$ and host $(I)$. Major under-represented regions in the mesocosm plant-selected datasets are indicated numerically or as $\mathrm{pRL} 11_{1}$. 


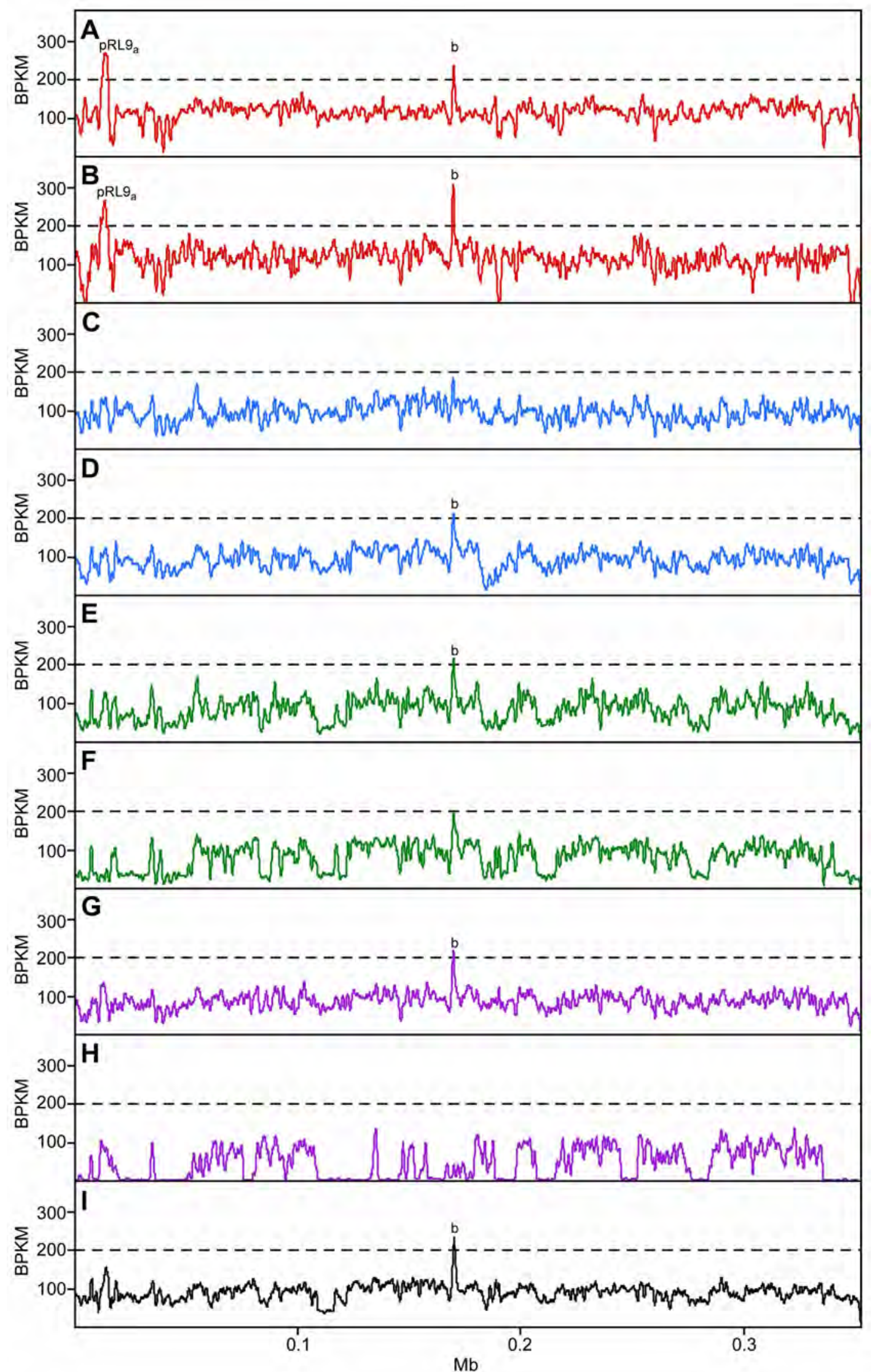

Supplementary Figure 3.S3. Conservation of the plasmid pRL9 of $R$. leguminosarum bv. viciae 3841 reference genome in the mesocosm plant-selected subpopulations. Coverage (BPKM) of genome regions along pRL9 by Bowtie2 recruited reads from pea $(A)$, pea $_{B}(B)$, lentil $_{A}(C)$, lentil $B(D)$, fava bean $A(E)$, fava bean $B(F)$, vetch betch $_{A}(G)$, vetch $(H)$ and host $(I)$. Major over-represented regions in the mesocosm plant-selected datasets are indicated by a lowercase letter or as $\mathrm{pRL} 9_{\mathrm{a}}$. 

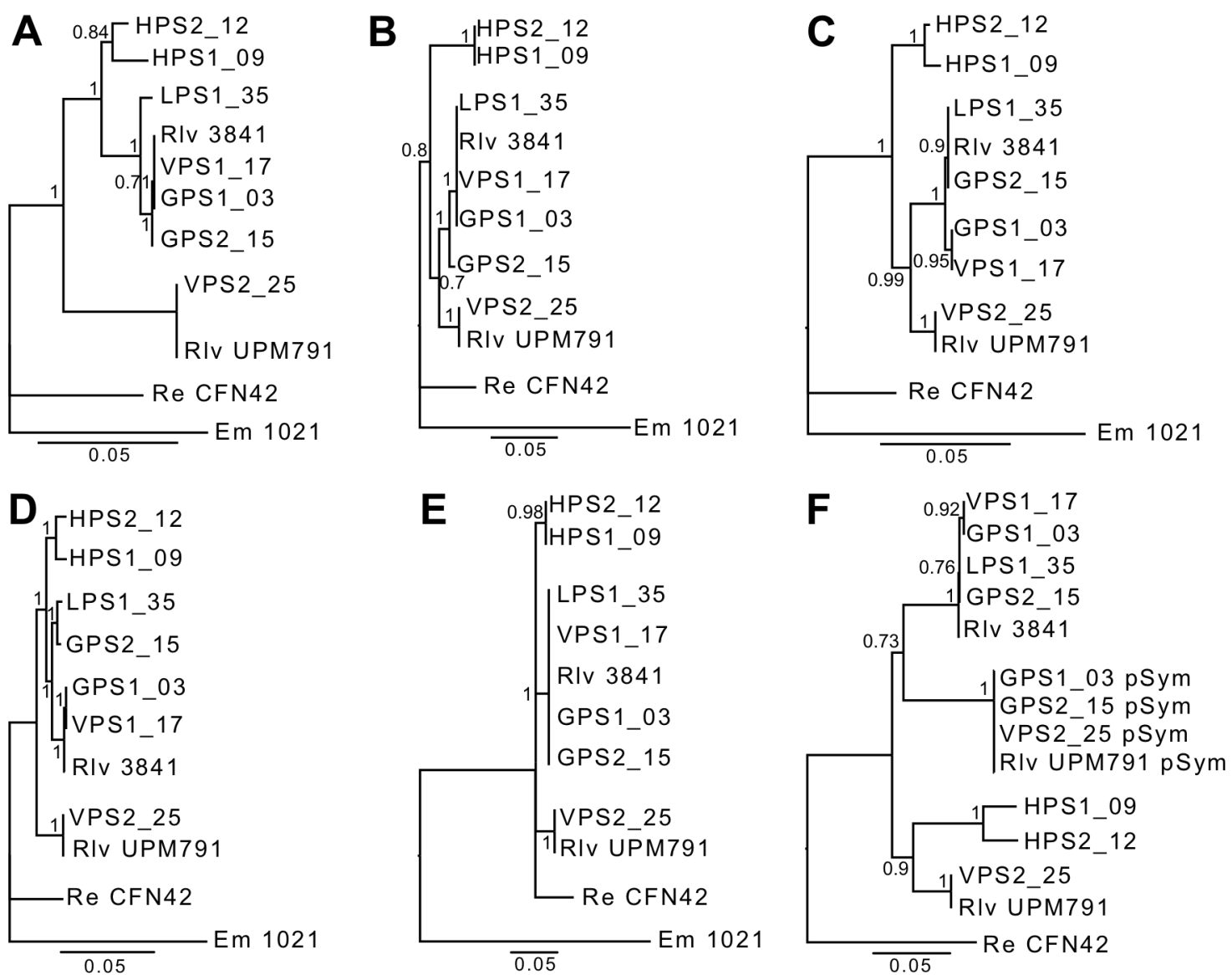

Supplementary Figure 3.S4. Phylogenetic trees based on housekeeping and chromosomal "symbiosis-related" genes. A (atpD), B ( $g / n l l), \mathrm{C}(\operatorname{rec} A), \mathrm{D}(r p o B), \mathrm{E}(r o s R)$ and F $(f n r N)$. Trees were constructed by the neighbor-joining method from multiple alignments. The significance of each branch is indicated by a bootstrap value calculated for 1,000 subsets, nodes with bootstrap values lower than 0.7 were collapsed. Abbreviations: RIv (Rhizobium leguminosarum bv. viciae), $\operatorname{Re}(R$. etli) and Em (Ensifer meliloti). 

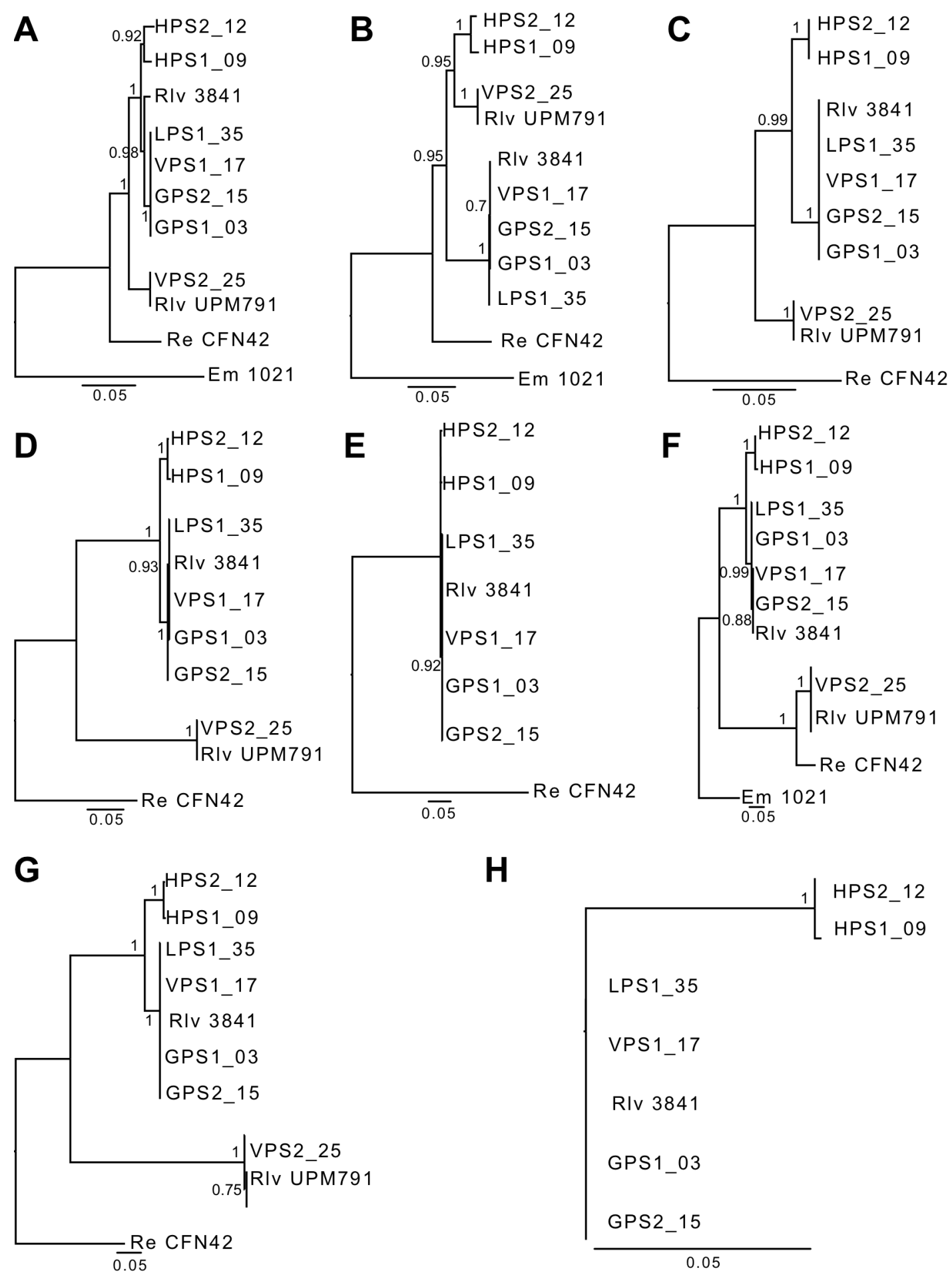

Supplementary Figure 3.S5. Phylogenetic trees based on "rhizosphere" and mesocosmselected genes. A (cheA), B (fabF1), C (pssD), D (plyA), E (dctM), F (pRL110330), G (RL2862) and H (RL1858). Trees were constructed by the neighbor-joining method from multiple alignments. The significance of each branch is indicated by a bootstrap value calculated for 1,000 subsets, nodes with bootstrap values lower than 0.7 were collapsed. Abbreviations: Rlv (Rhizobium leguminosarum bv. viciae), Re (R. etli) and Em (Ensifer meliloti). 
A

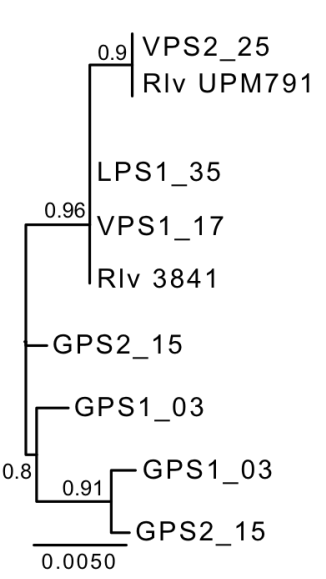

B

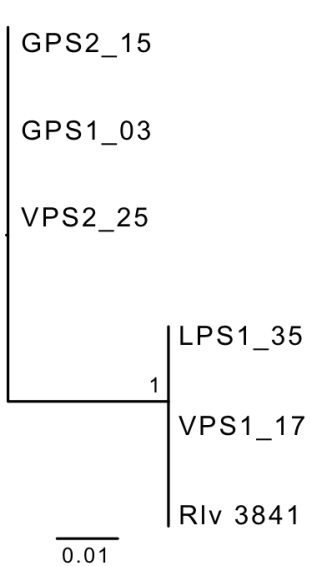

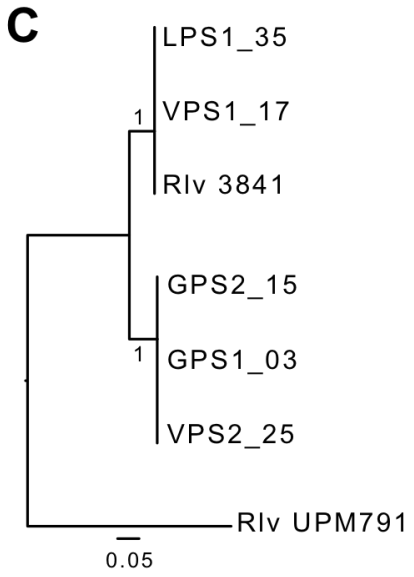

D

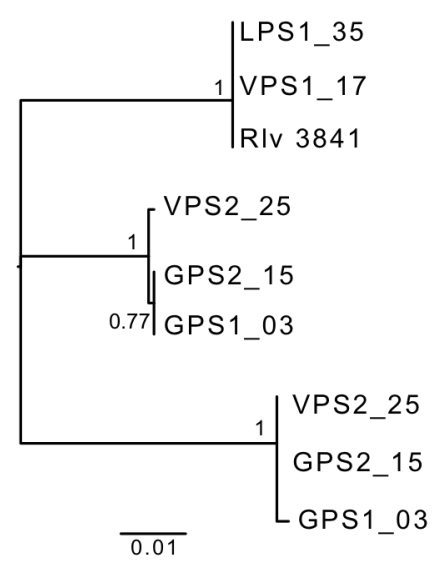

E

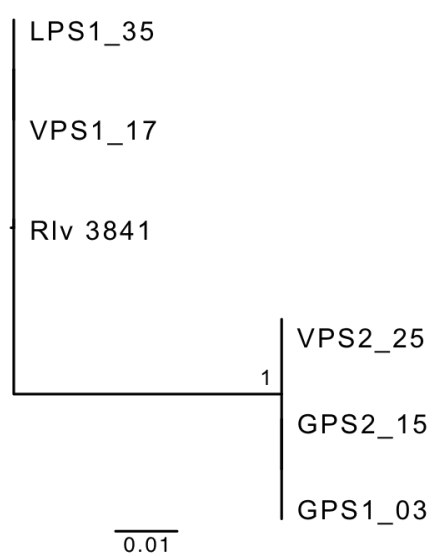

Supplementary Figure 3.S6. Phylogenetic trees based on mesocosm-selected genes. A (qat6X), B (pRL100131), C (attL), D (pRL100104) and E (pRL100107). Trees were constructed by the neighbor-joining method from multiple alignments. The significance of each branch is indicated by a bootstrap value calculated for 1,000 subsets, nodes with bootstrap values lower than 0.7 were collapsed. Abbreviation: Rlv (Rhizobium leguminosarum bv. viciae). 

7. GENERAL DISCUSSION 

Rhizobia are minor components of the soil microbiota (Jorrín and Imperial, 2015a). Therefore, attempts to approach the genomic structure of rhizobial populations in the soil with available metagenomic methodologies will be unsuccessful, and necessarily require a preliminary enrichment. This enrichment can not only be tedious but, more importantly, can introduce bias in the enriched population. Traditional enrichment methods have relied on the exquisite sensitivity and specificity of the legume-rhizobial symbiosis, using plants to specifically "trap" the small numbers of rhizobia present in soil within the legume nodules. The main objectives of this Thesis have centred, in a way, on the characterization of this bias that the plant host can impose on the rhizobial population being selected, using the Rhizobium leguminosarum bv. viciae-legume host system as a model. In order to study the bases of this bias (or, more properly, the host preference for specific rhizobial genotypes) a population genomics approach (PoolSeq) was chosen and implemented. This methodology takes advantage of the existence of a well-annotated $R$. leguminosarum bv. viciae reference genome (Young et al., 2006). The use of a reference genome for our studies imposed limitations that were already known and assumed beforehand: given the large plasticity of bacterial genomes (Flores et al., 2000, Mavingui et al., 2002, Jorrín and Imperial, 2015a), especially those living in soil, a complex and changing environment to which bacteria adapt by having very large genomes (Mitsui et al., 1997, Saito et al., 1998), we expected to find that a good number of the genes carried in the genome of any isolate would be missing in the reference genome and, therefore, they would be left out of our analyses. This was indeed the case (Chapter 1). However, the data presented in this Thesis show that it would be wrong to think that host preference for a specific genotype implies hundreds or even thousands of genes. In fact, when representative, hostselected isolates were sequenced, the number of exclusive genes was low (Chapter 3, Section 6.2.2.9). In addition, the bias of using a given reference genome in our analysis was also addressed by using other, closely-related genomes as reference. Despite the diversity observed among these reference genomes, the same overall tendencies were observed and general conclusions drawn (Chapter 2, section 5.2.11).

This Thesis represents the first use of the Pool-Seq methodology for rhizobial population genomics studies, and emphasizes the many possibilities it offers, especially in view of the dwindling cost of the new generation sequencing to genomically compare different populations without the effort and expense associated with sequencing and the bioinformatic management of a large number of genomes from the different subpopulations. In our case, this accounted for a total of 718 genomes (400 from the initial host subpopulations, 118 from the soil population, and 200 from mesocosm host subpopulations) that were studied with 13 Pool-Seq samples 
and the individual sequencing of just 7 genomes. This allowed a truly genomic analysis, well above in breadth, scope, and lack of bias, than any molecular marker specific methodology.

It is important to emphasize that the research problem central to this Thesis absolutely required a genomics / population genomics approach. Symbiotic specificity in the rhizobial-legume synthesis is commonly determined by the early exchange of signal molecules, such as the Nod factors. In our model, however, a single set of rhizobial symbiotic genes allows the establishment of successful symbioses with all the members of the Fabeae (Pisum, Lens, Vicia, Lathyrus and probably -although this has not been tested by us- Vavilovia), and thus the plant preference for specific genotypes must reflect other, more subtle and diverse physiological or biochemical properties, probably implicating life and development of rhizobia in the rhizosphere of the plant. Since the determinants of, for instance, rhizospheric fitness have not been well characterized, any approximation to this problem will benefit from genomic approaches. We were able to demonstrate that the different plant hosts employed in this Thesis ( $P$. sativum, L. culinaris, $V$. faba and $V$. sativa) selected different genotypes from those available in the P1 soil, and that the basis of selection was different for different plants. Pea and fava bean plants strongly selected for specific genotypes, but in opposite ways. Pea nodules were colonized by strains endowed with a large set of genes probably implicated in rhizospheric fitness, irrespective of the symbiotic genotype that harboured. This suggestion should be confirmed by in situ transcriptomic studies that, unfortunately, fall outside the scope of this work. However, fava bean plants restricted their selection to a specific symbiotic genotype that was not always localized in the same symbiotic plasmid, or within the same chromosomal background. No hard conclusions could be obtained for vetch, although we suggest that this plant might behave dually, either as a selective host if a genotype resulting in a rhizospheric advantage infected roots (such as in vetch ${ }_{B}$ subpopulation), or as a non-selective host, reflecting in its nodules the genotypic diversity present in soil (such as in vetch $_{\mathrm{A}}$ subpopulation). This last case was the situation found for lentils; the three subpopulations isolated from lentil nodules (initial lentil, lentil $\mathrm{A}_{\mathrm{A}}$ and lentil $\mathrm{B}_{\mathrm{B}}$ ) did not significantly differ among themselves regardless of the number of plant selection cycles, or with respect to the initial soil population.

Characterization of the soil population was determinant for our work and, we argue, for any work dealing with genotypic selection by the legume host. This is because soil is the source of the legume microsymbionts, and therefore any results will be dependent on the diversity and abundance of the available $R$. leguminosarum bv. viciae genotypes in the soil, in our case P1 soil. We further argue that any rhizobial 
population genomic study would be incomplete if not accompanied by a study of the native soil rhizobial population. Just as an example, we would not have been able to conclude that lentil is a non-selective host, or that peas selected for a genotype that was a minor component in the soil population unless we had previously characterized this population. Furthermore, characterizing soil rhizobia independently of their plant symbiotic phenotypes allowed us to uncover a large and diverse population of nonsymbiotic rhizobia whose existence had been known, but whose size and variability had not been studied. This "invisible" rhizobial population is, nonetheless, important for two reasons: a) they can be donors or recipients of genetic material to or from symbiotic rhizobia in horizontal gene transfer events, thus acting as an important pangenomic reservoir; and b) they can influence plant nutrition, growth and development not only of compatible legumes, but also of many other plants, in what is being uncovered as a major new role of non-symbiotic rhizobia as widespread constituents of plant microbiomes (Lundberg et al., 2012, Chaparro et al., 2013, Ottesen et al., 2013, Schlaeppi et al., 2013, Tkacz et al., 2015, Yeoh et al., 2015). 

8. OUTLOOK 

Although the overall goals of the Thesis were attained, numerous questions arose during its development that will require further work for their clarification.

We managed to ascertain the behaviour of most the hosts employed regarding selection of specific rhizobial genotypes. However, no hard conclusions were obtained for vetch, and repeating the mesocosm experiments in order to determine if vetches behave as lentil or as pea plants, or maybe dually, would be the first experiment to perform to close this project.

The next question to answer is the surprising inability of three mesocosm-selected strains, GPS1_03, GPS2_15 and VPS2_25, to form nodules with fava bean. Competition studies for nodulation of different hosts between mesocosm-selected strains should be carried out, together with an in-depth genomic analysis, comparing the genomes of mesocosm-selected strains that can and cannot nodulate fava bean. This should yield sets of candidate genes for each group that might be responsible for this phenotype.

As it was mentioned in the general discussion, in situ transcriptomic analyses of mesocosm-selected strains in the rhizosphere of their host should allow identification of set of genes relevant for root colonization. In view of the results reported, this should be especially interesting for pea-selected strains.

As concluded in this Thesis, host-mediated selection of genotypes is determined by the soil, which defines the abundance and diversity of genotypes that are able to infect these plants. In order to extend and generalize our results, similar experimentation should be carried out for other soils.

Finally, this Thesis shows that the Pool-Seq methodology, first used for Drosophila melanogaster, and also applied to other diploid species, can be used advantageously for bacterial population genomics, a haploid model. In order to confirm the potential of this methodology, as applied to haploid systems, it will be necessary to extend it to it other bacterial systems. 



\section{CONCLUSIONS}



1. A population genomics methodology was developed that allows to comprehensively study the bases of plant host preference for specific rhizobial genotypes in the Rhizobium leguminosarum-host legumes system.

2. The plant hosts employed (Pisum sativum, Lens culinaris, Vicia faba and V. sativa) selected different $R$. leguminosarum genotypes from those available in $\mathrm{P} 1$ soil.

2.1. Pea plants behaved as a selective host for certain rhizosphere-related rhizobial genotypes, independently of the symbiotic genotype that they harboured.

2.2. Fava bean behaved as a selective host for a specific symbiotic genotype. This symbiotic genotype could be localized in different symbiotic plasmids and chromosomal backgrounds.

2.3. Lentils were non-selective hosts, and their nodules reflected the rhizobial genotypic diversity present in the native soil population.

3. The native $R$. leguminosarum soil population determines the nature and diversity of the host-selected populations. Rhizobial soil populations include a large number of non-symbiotic rhizobia that constitute an uncharacterized pangenomic reservoir. 

"The fundamental laws of thermodynamics will place fixed limits on technological innovation and human advancement. In an isolated system, the entropy can only increase. A species set on endless growth is unsustainable."

Unsustainable The $2^{\text {nd }}$ Law Muse 

10. BIBLIOGRAPHY 

Abe M, Kawamura R, Higashi S, Mori S, Shibata M, Uchiumi T. 1998. Transfer of the symbiotic plasmid from Rhizobium leguminosarum biovar trifolii to Agrobacterium tumefaciens. The Journal of General and Applied Microbiology, 44: 65-74.

Acosta J, Eguiarte L, Santamaria R, Bustos P, Vinuesa P, Martinez-Romero E, Davila G, Gonzalez V. 2011. Genomic lineages of Rhizobium etli revealed by the extent of nucleotide polymorphisms and low recombination. BMC Evolutionary Biology, 11: 305.

Adékambi T, Drancourt M, Raoult D. 2009. The $r p o B$ gene as a tool for clinical microbiologists. Trends in Microbiology, 17: 37-45.

Adékambi T, Shinnick TM, Raoult D, Drancourt M. 2008. Complete rpo $B$ gene sequencing as a suitable supplement to DNA-DNA hybridization for bacterial species and genus delineation. International Journal of Systematic and Evolutionary Microbiology, 58: 1807-1814.

Aird D, Ross M, Chen W-S, Danielsson M, Fennell T, Russ C, Jaffe D, Nusbaum C, Gnirke A. 2011. Analyzing and minimizing PCR amplification bias in Illumina sequencing libraries. Genome Biology, 12: R18.

Akhtar MS, Siddiqui ZA. 2008. Biocontrol of a root-rot disease complex of chickpea by Glomus intraradices, Rhizobium sp. and Pseudomonas straita. Crop Protection, 27: 410-417.

Alami Y, Achouak W, Marol C, Heulin T. 2000. Rhizosphere Soil Aggregation and Plant Growth Promotion of Sunflowers by an Exopolysaccharide-Producing Rhizobium sp. Strain Isolated from Sunflower Roots. Applied and Environmental Microbiology, 66: 3393-3398.

Albareda M, Manyani H, Imperial J, Brito B, Ruiz-Argueso T, Bock A, Palacios JM. 2012. Dual role of HupF in the biosynthesis of [NiFe] hydrogenase in Rhizobium leguminosarum. BMC Microbiology, 12.

Alloing G, Travers I, Sagot B, Le Rudulier D, Dupont L. 2006. Proline betaine uptake in Sinorhizobium meliloti: Characterization of Prb, an Opp-Like ABC transporter regulated by both proline betaine and salinity stress. Journal of Bacteriology, 188: 6308-6317.

Altschul SF, Gish W, Miller W, Myers EW, Lipman DJ. 1990. Basic local alignment search tool. Journal of Molecular Biology, 215: 403-410.

Anderson AJ, Dawes EA. 1990. Occurrence, metabolism, metabolic role, and industrial uses of bacterial polyhydroxyalkanoates. Microbiological Reviews, 54: 450-472.

Ando S, Goto M, Meunchang S, Thongra-ar P, Fujiwara T, Hayashi H, Yoneyama T. 2005. Detection of nifH sequences in sugarcane (Saccharum officinarum L.) and Pineapple (Ananas comosus [L.] Merr.). Soil Science \& Plant Nutrition, 51: 303-308.

Angiuoli S, Matalka M, Gussman A, Galens K, Vangala M, Riley D, Arze C, White J, White O, Fricke WF. 2011. CloVR: a virtual machine for automated and portable sequence analysis from the desktop using cloud computing. BMC Bioinformatics, 12: 356.

Arfaoui A, Sifi B, Boudabous A, El Hadrami I, Cherif M. 2006. Identification of Rhizobium isolates possessing antagonistic activity against Fusarium oxysporum f.sp ciceris, the causal agent of Fusarium wilt of chickpea. Journal of Plant Pathology, 88: 67-75.

Aslam SN, Newman M-A, Erbs G, Morrissey KL, Chinchilla D, Boller T, Jensen TT, De Castro C, lerano T, Molinaro A, Jackson RW, Knight MR, Cooper RM. 2008. Bacterial polysaccharides suppress induced innate immunity by calcium chelation. Current Biology, 18: 1078-1083.

Assefa S, Keane TM, Otto TD, Newbold C, Berriman M. 2009. ABACAS: algorithm-based automatic contiguation of assembled sequences. Bioinformatics, 25: 1968-1969.

Baginsky C, Brito B, Imperial J, Palacios JM, Ruiz-Argueso T. 2002. Diversity and evolution of hydrogenase systems in rhizobia. Applied and Environmental Microbiology, 68: 49154924.

Bailly X, Giuntini E, Sexton MC, Lower RPJ, Harrison PW, Kumar N, Young JPW. 2011. Population genomics of Sinorhizobium medicae based on low-coverage sequencing of sympatric isolates. ISME Journal, 5: 1722-1734.

Ballard R. 2004. Size, symbiotic effectiveness and genetic diversity of field pea rhizobia (Rhizobium leguminosarum bv. viciae) populations in South Australian soils. Soil Biology \& Biochemistry, 36: 1347-1355.

Banfalvi Z, Kondorosi A. 1989. Production of root hair deformation factors by Rhizobium meliloti nodulation genes in Escherichia coli: $\mathrm{HsnD}(\mathrm{NodH})$ is involved in the plant hostspecific modification of the NodABC factor. Plant Molecular Biology, 13: 1-12. 
Bankevich A, Nurk S, Antipov D, Gurevich AA, Dvorkin M, Kulikov AS, Lesin VM, Nikolenko SI, Pham S, Prjibelski AD, Pyshkin AV, Sirotkin AV, Vyahhi N, Tesler G, Alekseyev MA, Pevzner PA. 2012. SPAdes: a new genome assembly algorithm and its applications to single-cell sequencing. Journal of Computational Biology, 19: 455477.

Barber LE. 1979. Use of selective agents for recovery of Rhizobium meliloti from soil. Soil Science Society of America Journal, 43.

Barreiro LB, Laval G, Quach H, Patin E, Quintana-Murci L. 2008. Natural selection has driven population differentiation in modern humans. Nature Genetics, 40: 340-345.

Bassam B, Rolfe B, Djordjevic M. 1986. Macroptilium atropurpureum (siratro) host specificity genes are linked to a nodD-like gene in the broad host range Rhizobium strain NGR234. Molecular and General Genetics MGG, 203: 49-57.

Baumann U, Wu S, Flaherty KM, McKay DB. 1993. Three-dimensional structure of the alkaline protease of Pseudomonas aeruginosa: a two-domain protein with a calcium binding parallel beta roll motif. The EMBO Journal, 12: 3357-3364.

Bender GL, Nayudu M, Strange KKL, Rolfe BG. 1988. The nodD1 gene from Rhizobium strain NGR234 is a key determinant in the extension of host range to the non legume Parasponia. Molecular Plant-Microbe Interactions, 1: 259-266.

Benhizia Y, Benhizia H, Benguedouar A, Muresu R, Giacomini A, Squartini A. 2004. Gamma proteobacteria can nodulate legumes of the genus Hedysarum. Systematic and Applied Microbiology, 27: 462-468.

Berge O, Lodhi A, Brandelet G, Santaella C, Roncato MA, Christen R, Heulin T, Achouak W. 2009. Rhizobium alamii sp nov., an exopolysaccharide-producing species isolated from legume and non-legume rhizospheres. International Journal of Systematic and Evolutionary Microbiology, 59: 367-372.

Beringer JE. 1974. R Factor Transfer in Rhizobium leguminosarum. Microbiology, 84: 188-198.

Bernèche-D'Amours A, Ghinet MG, Beaudin J, Brzezinski R, Roy S. 2011. Sequence analysis of $r p o B$ and $r p o D$ gene fragments reveals the phylogenetic diversity of actinobacteria of genus Frankia. Canadian Journal of Microbiology, 57: 244-249.

Bertani G, Weigle JJ. 1953. Host controlled variation in bacterial viruses. Journal of Bacteriology, 65: 113-121.

Bhattacharjee RB, Jourand P, Chaintreuil C, Dreyfus B, Singh A, Mukhopadhyay SN. 2011. Indole acetic acid and ACC deaminase-producing Rhizobium leguminosarum bv. trifolii SN10 promote rice growth, and in the process undergo colonization and chemotaxis. Biology and Fertility of Soils, 48: 173-182.

Bolger AM, Lohse M, Usadel B. 2014. Trimmomatic: a flexible trimmer for lllumina sequence data. Bioinformatics.

Boncompagni E, Østerås M, Poggi M-C, le Rudulier D. 1999. Occurrence of choline and glycine betaine uptake and metabolism in the family Rhizobiaceae and their roles in osmoprotection. Applied and Environmental Microbiology, 65: 2072-2077.

Bond D. 1976. Field bean (V. faba). In: Evolution of Crop Plants. In: Simmonds N ed. London, Longman and Hall.

Boscari A, Mandon K, Dupont L, Poggi M-C, Le Rudulier D. 2002. BetS is a major glycine betaine/proline betaine transporter required for early osmotic adjustment in Sinorhizobium meliloti. Journal of Bacteriology, 184: 2654-2663.

Boussau B, Karlberg EO, Frank AC, Legault B-A, Andersson SGE. 2004. Computational inference of scenarios for a-proteobacterial genome evolution. Proceedings of the National Academy of Sciences, 101: 9722-9727.

Bresson J, Varoquaux F, Bontpart T, Touraine B, Vile D. 2013. The PGPR strain Phyllobacterium brassicacearum STM196 induces a reproductive delay and physiological changes that result in improved drought tolerance in Arabidopsis. New Phytologist, 200: 558-569.

Brewin NJ, Beringer JE, Johnston AWB. 1980. Plasmid-mediated transfer of host-range specificity between two strains of Rhizobium leguminosarum. Microbiology, 120: 413420.

Brito B, Palacios JM, Hidalgo E, Imperial J, Ruizargueso T. 1994. Nickel availability to pea (Pisum sativum L.) plants limits hydrogenase activity of Rhizobium leguminosarum bv. viciae bacteroids by affecting the processing of the hydrogenase structural subunits. Journal of Bacteriology, 176: 5297-5303. 
Brockman FJ, Bezdicek DF. 1989. Diversity within serogroups of Rhizobium leguminosarum biovar viceae in the Palouse region of Eastern Washington as indicated by plasmid profiles, intrinsic antibiotic resistance, and topography. Applied and Environmental Microbiology, 55: 109-115.

Brockwell J. 1963. Accuracy of a plant-infection technique for counting populations of Rhizobium trifolii. Applied Microbiology, 11: 377-\&.

Broghammer A, Krusell L, Blaise M, Sauer J, Sullivan JT, Maolanon N, Vinther M, Lorentzen A, Madsen EB, Jensen KJ, Roepstorff P, Thirup S, Ronson CW, Thygesen MB, Stougaard J. 2012. Legume receptors perceive the rhizobial lipochitin oligosaccharide signal molecules by direct binding. Proceedings of the National Academy of Sciences, 109: 13859-13864.

Bromfield ESP, Barran LR, Wheatcroft R. 1995. Relative genetic structure of a population of Rhizobium meliloti isolated directly from soil and from nodules of alfalfa (Medicago sativa) and sweet clover (Melilotus alba). Molecular Ecology, 4: 183-188.

Bromfield ESP, Wheatcroft R, Barran LR. 1994. Medium for direct isolation of Rhizobium meliloti from soils. Soil Biology \& Biochemistry, 26: 423-428.

Broughton W, Zhang F, Perret X, Staehelin C. 2003. Signals exchanged between legumes and Rhizobium: agricultural uses and perspectives. Plant and Soil, 252: 129-137.

Brown SD, Utturkar SM, Klingeman DM, Johnson CM, Martin SL, Land ML, Lu T-YS, Schadt CW, Doktycz MJ, Pelletier DA. 2012. Twenty-One genome sequences from Pseudomonas species and 19 genome sequences from diverse bacteria isolated from the rhizosphere and endosphere of Populus deltoides. Journal of Bacteriology, 194: 5991-5993.

Bryan NS, van Grinsven H. 2013. Chapter Three - The Role of Nitrate in Human Health. In: Donald LS ed. Advances in Agronomy. Academic Press.

Canfield DE, Glazer AN, Falkowski PG. 2010. The evolution and future of Earth's nitrogen cycle. Science, 330: 192-196.

Carlier A, Chevrot R, Dessaux Y, Faure D. 2004. The assimilation of $Y$-butyrolactone in Agrobacterium tumefaciens C58 interferes with the accumulation of the $\mathrm{N}$-acylhomoserine lactone signal. Molecular Plant-Microbe Interactions, 17: 951-957.

Case RJ, Boucher Y, Dahllöf I, Holmström C, Doolittle WF, Kjelleberg S. 2007. Use of 16S rRNA and $r p o B$ genes as molecular markers for microbial ecology studies. Applied and Environmental Microbiology, 73: 278-288.

Cesco S, Mimmo T, Tonon G, Tomasi N, Pinton R, Terzano R, Neumann G, Weisskopf L, Renella G, Landi L, Nannipieri P. 2012. Plant-borne flavonoids released into the rhizosphere: impact on soil bio-activities related to plant nutrition. A review. Biology and Fertility of Soils, 48: 123-149.

Chaban B, Links M, Jayaprakash T, Wagner E, Bourque D, Lohn Z, Albert A, van Schalkwyk J, Reid G, Hemmingsen S, Hill J, Money D. 2014. Characterization of the vaginal microbiota of healthy Canadian women through the menstrual cycle. Microbiome, 2: 23.

Chaparro JM, Badri DV, Vivanco JM. 2013. Rhizosphere microbiome assemblage is affected by plant development. ISME Journal.

Colebatch G, Desbrosses G, Ott T, Krusell L, Montanari O, Kloska S, Kopka J, Udvardi MK. 2004. Global changes in transcription orchestrate metabolic differentiation during symbiotic nitrogen fixation in Lotus japonicus. The Plant Journal, 39: 487-512.

Cooper JE. 2004. Multiple responses of rhizobia to flavonoids during legume root infection. In: Callow JA ed. Advances in Botanical Research. Academic Press.

Cooper JE. 2007. Early interactions between legumes and rhizobia: disclosing complexity in a molecular dialogue. Journal of Applied Microbiology, 103: 1355-1365.

Crabtree J, Angiuoli S, Wortman J, White 0. 2007. Sybil: methods and software for multiple genome comparison and visualization. In: Ochs M ed. Gene Function Analysis. Humana Press.

Crook MB, Lindsay DP, Biggs MB, Bentley JS, Price JC, Clement SC, Clement MJ, Long SR, Griffitts JS. 2012. Rhizobial plasmids that cause impaired symbiotic nitrogen fixation and enhanced host invasion. Molecular Plant-Microbe Interactions, 25: 10261033.

Curatti L, Rubio LM. 2014. Challenges to develop nitrogen-fixing cereals by direct nif-gene transfer. Plant Science, 225: 130-137. 
Davis EO, Evans IJ, Johnston AWB. 1988. Identification of nodX, a gene that allows Rhizobium leguminosarum biovar viciae strain TOM to nodulate Afghanistan peas. Molecular and General Genetics MGG, 212: 531-535.

de Almeida A, Nikel PI, Giordano AM, Pettinari MJ. 2007. Effects of granule-associated protein PhaP on glycerol-dependent growth and polymer production in poly(3hydroxybutyrate)-producing Escherichia coli. Applied and Environmental Microbiology, 73: 7912-7916.

De Hoff P, Brill L, Hirsch A. 2009. Plant lectins: the ties that bind in root symbiosis and plant defense. Molecular Genetics and Genomics, 282: 1-15.

Deakin WJ, Broughton WJ. 2009. Symbiotic use of pathogenic strategies: rhizobial protein secretion systems. Nat Rev Micro, 7: 312-320.

Delepelaire P. 2004. Type I secretion in gram-negative bacteria. Biochimica et Biophysica Acta, 1694: $149-161$

Delmont TO, Robe P, Cecillon S, Clark IM, Constancias F, Simonet P, Hirsch PR, Vogel TM. 2011. Accessing the soil metagenome for studies of microbial diversity. Applied and Environmental Microbiology, 77: 1315-1324.

Denarie J, Debelle F, Prome JC. 1996. Rhizobium lipo-chitooligosaccharide nodulation factors: signaling molecules mediating recognition and morphogenesis. Annual Review Biochemistry, 65: 503-35.

Denison RF, Kiers ET. 2004. Lifestyle alternatives for rhizobia: mutualism, parasitism, and forgoing symbiosis. FEMS Microbiology Letters, 237: 187-193.

Depret G, Houot S, Allard MR, Breuil MC, Nouaim R, Laguerre G. 2004. Long-term effects of crop management on Rhizobium leguminosarum biovar viciae populations. FEMS Microbiology Ecology, 51: 87-97.

Downie JA. 2010. The roles of extracellular proteins, polysaccharides and signals in the interactions of rhizobia with legume roots. FEMS Microbiology Reviews, 34: 150-170.

Downie JA, Surin BP. 1990. Either of two nod gene loci can complement the nodulation defect of a nod deletion mutant of Rhizobium leguminosarum bv viciae. Molecular and General Genetics MGG, 222: 81-86.

Doyle JJ, Luckow MA. 2003. The Rest of the Iceberg. Legume Diversity and Evolution in a Phylogenetic Context. Plant Physiology, 131: 900-910.

Drew EA, Denton MD, Sadras VO, Ballard RA. 2012. Agronomic and environmental drivers of population size and symbiotic performance of Rhizobium leguminosarum bv. viciae in Mediterranean-type environments. Crop \& Pasture Science, 63: 467-477.

Dupont L, Garcia I, Poggi M-C, Alloing G, Mandon K, Le Rudulier D. 2004. The Sinorhizobium meliloti ABC transporter Cho is highly specific for choline and expressed in bacteroids from Medicago sativa nodules. Journal of Bacteriology, 186: 5988-5996.

Dylan T, lelpi L, Stanfield S, Kashyap L, Douglas C, Yanofsky M, Nester E, Helinski DR, Ditta G. 1986. Rhizobium meliloti genes required for nodule development are related to chromosomal virulence genes in Agrobacterium tumefaciens. Proceedings of the National Academy of Sciences, 83: 4403-4407.

Economou A, Davies AE, Johnston AWB, Downie JA. 1994. The Rhizobium leguminosarum biovar viciae nodO gene can enable a nodE mutant of Rhizobium leguminosarum biovar trifolii to nodulate vetch. Microbiology, 140: 2341-2347.

Economou A, Hamilton WD, Johnston AW, Downie JA. 1990. The Rhizobium nodulation gene nodO encodes a $\mathrm{Ca} 2(+)$-binding protein that is exported without $\mathrm{N}$-terminal cleavage and is homologous to haemolysin and related proteins. The EMBO Journal, $\mathbf{9}$ : 349-54.

Edgar RC. 2004. MUSCLE: multiple sequence alignment with high accuracy and high throughput. Nucleic Acids Research, 32: 1792-1797.

Ellwood M, Nomura M. 1980. Deletion of a ribosomal ribonucleic acid operon in Escherichia coli. Journal of Bacteriology, 143: 1077-1080.

Erisman JW, Sutton MA, Galloway J, Klimont Z, Winiwarter W. 2008. How a century of ammonia synthesis changed the world. Nature Geoscience, 1: 636-639.

Evans J, Gregory A, Dobrowolski N, Morris SG, O'Connor GE, Wallace C. 1996. Nodulation of field-grown Pisum sativum and Vicia faba: competitiveness of inoculant strains of Rhizobium leguminosarum bv. viciae determined by an indirect, competitive ELISA method. Soil Biology \& Biochemistry, 28: 247-255.

Fåhraeus G. 1957. The infection of clover root hairs by nodule bacteria studied by a simple glass slide technique. Microbiology, 16: 374-381. 
Fierer N, Schimel JP, Holden PA. 2003. Influence of drying-rewetting frequency on soil bacterial community structure. Microbial Ecology, 45: 63-71.

Finan TM, Hirsch AM, Leigh JA, Johansen E, Kuldau GA, Deegan S, Walker GC, Signer ER. 1985. Symbiotic mutants of Rhizobium meliloti that uncouple plant from bacterial differentiation. Cell, 40: 869-877.

Flores M, Mavingui P, Perret X, Broughton WJ, Romero D, Hernández G, Dávila G, Palacios R. 2000. Prediction, identification, and artificial selection of DNA rearrangements in Rhizobium: toward a natural genomic design. Proceedings of the National Academy of Sciences, 97: 9138-9143.

Fox GE, Pechman KR, Woese CR. 1977. Comparative cataloging of $16 \mathrm{~S}$ ribosomal ribonucleic acid: molecular approach to prokaryotic systematics. International Journal of Systematic and Evolutionary Microbiology, 27: 44-57.

Fox MA, Karunakaran R, Leonard ME, Mouhsine B, Williams A, East AK, Downie JA, Poole PS. 2008. Characterization of the quaternary amine transporters of Rhizobium leguminosarum bv. viciae 3841. FEMS Microbiology Letters, 287: 212-220.

Francis DD, Schepers JS, Vigil MF. 1993. Post-Anthesis Nitrogen Loss from Corn. Agronomy Journal, 85: 659-663.

Frank B. 1889. Ueber die Pilzsymbiose der Leguminosen. Berichte der Deutschen Botanischen Gesellschaft, 7: 332-346.

Friedland A, Relyea R, Courard-Hauri D. 2012. Ecosystem Ecology. Environmental Science for $A P^{*}$. W.H. Freeman and Company.

Frink CR, Waggoner PE, Ausubel JH. 1999. Nitrogen fertilizer: retrospect and prospect. Proceedings of the National Academy of Sciences, 96: 1175-1180.

Fuhrer T, Fischer E, Sauer U. 2005. Experimental identification and quantification of glucose metabolism in seven bacterial species. Journal of Bacteriology, 187: 1581-1590.

Futschik A, Schloetterer C. 2010. The next generation of molecular markers from massively parallel sequencing of pooled DNA samples. Genetics, 186: 207-218.

Gage DJ. 2004. Infection and invasion of roots by symbiotic, nitrogen-fixing rhizobia during nodulation of temperate legumes. Microbiology and Molecular Biology Reviews, 68: 280-+.

Galardini M, Mengoni A, Brilli M, Pini F, Fioravanti A, Lucas S, Lapidus A, Cheng JF, Goodwin L, Pitluck S, Land M, Hauser L, Woike T, Mikhailova N, Ivanova N, Daligault H, Bruce D, Detter C, Tapia R, Han C, Teshima H, Mocali S, Bazzicalupo M, Biondi EG. 2011. Exploring the symbiotic pangenome of the nitrogen-fixing bacterium Sinorhizobium meliloti. BMC Genomics, 12.

Galloway JN, Townsend AR, Erisman JW, Bekunda M, Cai Z, Freney JR, Martinelli LA, Seitzinger SP, Sutton MA. 2008. Transformation of the nitrogen cycle: recent trends, questions, and potential solutions. Science, 320: 889-892.

García-Fraile P, Rivas R, Willems A, Peix A, Martens M, Martínez-Molina E, Mateos PF, Velázquez E. 2007. Rhizobium cellulosilyticum sp. nov., isolated from sawdust of Populus alba. International Journal of Systematic and Evolutionary Microbiology, 57: 844-848.

Gault RR, Schwinghamer EA. 1993. Direct isolation of Bradyrhizobium japonicum from soil. Soil Biology \& Biochemistry, 25: 1161-1166.

Geddes BA, Ryu M-H, Mus F, Garcia Costas A, Peters JW, Voigt CA, Poole P. 2015. Use of plant colonizing bacteria as chassis for transfer of N2-fixation to cereals. Current Opinion in Biotechnology, 32: 216-222.

Gould PS, Burgar HR, Lund PA. 2007. Homologous cpn60 genes in Rhizobium leguminosarum are not functionally equivalent. Cell Stress \& Chaperones, 12: 123-131.

Gourion B, Berrabah F, Ratet P, Stacey G. 2015. Rhizobium-legume symbioses: the crucial role of plant immunity. Trends in Plant Science, 20: 186-194.

Goyal V, Chetal S, Nainawatee HS. 1986. Alterations in Rhizobium trifolii catalase under water stress. Folia Microbiologica, 31: 164-166.

Graham PH. 1969. Selective medium for growth of Rhizobium. Applied Microbiology, 17: 769770.

Graham PH, Vance CP. 2003. Legumes: Importance and constraints to greater use. Plant Physiology, 131: 872-877.

Gurevich A, Saveliev V, Vyahhi N, Tesler G. 2013. QUAST: quality assessment tool for genome assemblies. Bioinformatics, 29: 1072-1075. 
Gutierrez D, Hernando Y, Palacios JM, Imperial J, RuizArgueso T. 1997. FnrN controls symbiotic nitrogen fixation and hydrogenase activities in Rhizobium leguminosarum biovar viciae UPM791. Journal of Bacteriology, 179: 5264-5270.

Gutiérrez RA. 2012. Systems biology for enhanced plant nitrogen nutrition. Science, 336: 16731675.

Handley BA, Hedges AJ, Beringer JE. 1998. Importance of host plants for detecting the population diversity of Rhizobium leguminosarum biovar viciae in soil. Soil Biology \& Biochemistry, 30: 241-249.

Harrison PW, Lower RPJ, Kim NKD, Young JPW. 2010. Introducing the bacterial 'chromid': not a chromosome, not a plasmid. Trends in Microbiology, 18: 141-148.

Hartmann A, Giraud JJ, Catroux G. 1998. Genotypic diversity of Sinorhizobium (formerly Rhizobium) meliloti strains isolated directly from a soil and from nodules of alfalfa (Medicago sativa) grown in the same soil. FEMS Microbiology Ecology, 25: 107-116.

Heberle H, Meirelles GV, da Silva FR, Telles GP, Minghim R. 2015. InteractiVenn: a webbased tool for the analysis of sets through Venn diagrams. BMC Bioinformatics, 16: 1-7.

Helgason T, Daniell TJ, Husband R, Fitter AH, Young JPW. 1998. Ploughing up the woodwide web? Nature, 394: 431-431.

Herridge DF, Peoples MB, Boddey RM. 2008. Global inputs of biological nitrogen fixation in agricultural systems. Plant and Soil, 311: 1-18.

Hirel B, Tétu T, Lea PJ, Dubois F. 2011. Improving nitrogen use efficiency in crops for sustainable agriculture. Sustainability, 3: 1452.

Hirsch AM. 1992. Developmental biology of legume nodulation. New Phytologist, 122: 211-237.

Hirsch PR. 1996. Population dynamics of indigenous and genetically modified rhizobia in the field. New Phytologist, 133: 159-171.

Horvath B, Kondorosi E, John M, Schmidt J, Török I, Györgypal Z, Barabas I, Wieneke U, Schell J, Kondorosi A. 1986. Organization, structure and symbiotic function of Rhizobium meliloti nodulation genes determining host specificity for alfalfa. Cell, 46: 335-343.

Hotter GS, Scott DB. 1991. Exopolysaccharide mutants of Rhizobium loti are fully effective on a determinate nodulating host but are ineffective on an indeterminate nodulating host. Journal of Bacteriology, 173: 851-859.

Hui D, Ling V. 2002. A combinatorial approach toward analyzing functional elements of the Escherichia coli hemolysin signal sequence. Biochemistry, 41: 5333-5339.

Hui D, Morden C, Zhang F, Ling V. 2000. Combinatorial analysis of the structural requirements of the Escherichia coli hemolysin signal sequence. Journal of Biological Chemistry, 275: 2713-2720.

Hyatt D, Chen G-L, LoCascio P, Land M, Larimer F, Hauser L. 2010. Prodigal: prokaryotic gene recognition and translation initiation site identification. BMC Bioinformatics, 11: 119.

Hynes MF, Oconnell MP. 1990. Host plant effect on competition among strains of Rhizobium leguminosarum. Canadian Journal of Microbiology, 36: 864-869.

Ibáñez F, Angelini J, Taurian T, Tonelli ML, Fabra A. 2009. Endophytic occupation of peanut root nodules by opportunistic Gammaproteobacteria. Systematic and Applied Microbiology, 32: 49-55.

Jarvis BDW, Ward LJH, Slade EA. 1989. Expression by soil bacteria of nodulation genes from Rhizobium leguminosarum biovar trifolii. Applied and Environmental Microbiology, 55: 1426-1434.

Johnson LA, Chaban B, Harding JC, Hill JE. 2015. Optimizing a PCR protocol for cpn60based microbiome profiling of samples variously contaminated with host genomic DNA. BMC Research Notes, 8: 253.

Johnston AWB, Beringer JE. 1975. Identification of the Rhizobium strains in pea root nodules using genetic markers. Microbiology, 87: 343-350.

Jones JDG, Dangl JL. 2006. The plant immune system. Nature, 444: 323-329.

Jorrín B, Imperial J. 2015a. Pool-Seq analysis of microsymbiont selection by the legume plant host. Biological Nitrogen Fixation. John Wiley \& Sons, Inc.

Jorrín B, Imperial J. 2015b. Population genomics analysis of legume host preference for specific rhizobial genotypes in the Rhizobium leguminosarum bv. viciae symbioses. Molecular Plant-Microbe Interactions, 28: 310-318. 
Kambara K, Ardissone S, Kobayashi H, Saad MM, Schumpp O, Broughton WJ, Deakin WJ. 2009. Rhizobia utilize pathogen-like effector proteins during symbiosis. Molecular Microbiology, 71: 92-106.

Karunakaran R, Ramachandran VK, Seaman JC, East AK, Mouhsine B, Mauchline TH, Prell J, Skeffington A, Poole PS. 2009. Transcriptomic analysis of Rhizobium leguminosarum biovar viciae in symbiosis with host plants Pisum sativum and Vicia cracca. Journal of Bacteriology, 191: 4002-4014.

Kawaguchi A. 2014. Reduction in pathogen populations at grapevine wound sites is associated with the mechanism underlying the biological control of crown gall by Rhizobium vitis strain ARK-1. Microbes and Environments, 29: 296-302.

Kempf B, Bremer E. 1998. Uptake and synthesis of compatible solutes as microbial stress responses to high-osmolality environments. Archives of Microbiology, 170: 319-330.

Kereszt A, Mergaert P, Kondorosi E. 2011. Bacteroid development in legume nodules: evolution of mutual benefit or of sacrificial victims? Molecular Plant-Microbe Interactions, 24: 1300-1309.

Khamis A, Colson P, Raoult D, Scola BL. 2003. Usefulness of $r p o B$ gene sequencing for identification of Afipia and Bosea species, including a strategy for choosing discriminative partial sequences. Applied and Environmental Microbiology, 69: 67406749.

Khamis A, Raoult D, La Scola B. 2005. Comparison between rpoB and 16S rRNA gene sequencing for molecular identification of 168 clinical isolates of Corynebacterium. Journal of Clinical Microbiology, 43: 1934-1936.

Kinkle BK, Sadowsky MJ, Johnstone K, Koskinen WC. 1994. Tellurium and selenium resistance in rhizobia and its potential use for direct isolation of Rhizobium meliloti from soil. Applied and Environmental Microbiology, 60: 1674-1677.

Kirzinger MWB, Stavrinides J. 2012. Host specificity determinants as a genetic continuum. Trends in Microbiology, 20: 88-93.

Klappenbach JA, Saxman PR, Cole JR, Schmidt TM. 2001. rrndb: the ribosomal RNA operon copy number database. Nucleic Acids Research, 29: 181-184.

Knietsch A, Waschkowitz T, Bowien S, Henne A, Daniel R. 2003. Metagenomes of complex microbial consortia derived from different soils as sources for novel genes conferring formation of carbonyls from short-chain polyols on Escherichia coli. Journal of Molecular Microbiology and Biotechnology, 5: 46-56.

Koboldt DC, Zhang QY, Larson DE, Shen D, McLellan MD, Lin L, Miller CA, Mardis ER, Ding L, Wilson RK. 2012. VarScan 2: somatic mutation and copy number alteration discovery in cancer by exome sequencing. Genome Research, 22: 568-576.

Koch M, Delmotte N, Rehrauer H, Vorholt JA, Pessi G, Hennecke H. 2010. Rhizobial adaptation to hosts, a new facet in the legume root-nodule symbiosis. Molecular PlantMicrobe Interactions, 23: 784-790.

Kofler R, Orozco-terWengel P, De Maio N, Pandey RV, Nolte V, Futschik A, Kosiol C, Schlotterer C. 2011a. PoPoolation: a toolbox for population genetic analysis of next generation sequencing data from pooled individuals. PLOS ONE, 6.

Kofler R, Pandey RV, Schlotterer C. 2011b. PoPoolation2: identifying differentiation between populations using sequencing of pooled DNA samples (Pool-Seq). Bioinformatics, 27: 3435-3436.

Kong Z, Glick BR, Duan J, Ding S, Tian J, McConkey BJ, Wei G. 2015. Effects of 1aminocyclopropane-1-carboxylate (ACC) deaminase-overproducing Sinorhizobium meliloti on plant growth and copper tolerance of Medicago Iupulina. Plant and Soil, 391: 383-398.

Korczak B, Christensen H, Emler S, Frey J, Kuhnert P. 2004. Phylogeny of the family Pasteurellaceae based on rpoB sequences. International Journal of Systematic and Evolutionary Microbiology, 54: 1393-1399.

Kucey RMN, Hynes MF. 1989. Populations of Rhizobium leguminosarum biovars phaseoli and viceae in fields after bean or pea in rotation with nonlegumes. Canadian Journal of Microbiology, 35: 661-667.

Kumar N, Lad G, Giuntini E, Kaye ME, Udomwong P, Shamsani NJ, Young JPW, Bailly X. 2015. Bacterial genospecies that are not ecologically coherent: population genomics of Rhizobium leguminosarum. Open Biology 5.

Kurtz S, Phillippy A, Delcher A, Smoot M, Shumway M, Antonescu C, Salzberg S. 2004. Versatile and open software for comparing large genomes. Genome Biology, 5: R12. 
Lagesen K, Hallin P, Rødland EA, Stærfeldt H-H, Rognes T, Ussery DW. 2007. RNAmmer: consistent and rapid annotation of ribosomal RNA genes. Nucleic Acids Research, 35: 3100-3108.

Laguerre G, Bardin M, Amarger N. 1993a. Isolation from soil of symbiotic and nonsymbiotic Rhizobium leguminosarum by DNA hybridization. Canadian Journal of Microbiology, 39: 1142-1149.

Laguerre G, Geniaux E, Mazurier SI, Casartelli RR, Amarger N. 1993b. Conformity and diversity among field isolates of Rhizobium leguminosarum bv. viciae, bv. trifolii, and bv. phaseoli revealed by DNA hybridization using chromosome and plasmid probes. Canadian Journal of Microbiology, 39: 412-419.

Laguerre G, Louvrier P, Allard MR, Amarger N. 2003. Compatibility of rhizobial genotypes within natural populations of Rhizobium leguminosarum biovar viciae for nodulation of host legumes. Applied and Environmental Microbiology, 69: 2276-2283.

Laguerre G, Mavingui P, Allard MR, Charnay MP, Louvrier P, Mazurier SI, RigottierGois L, Amarger N. 1996. Typing of rhizobia by PCR DNA fingerprinting and PCR-restriction fragment length polymorphism analysis of chromosomal and symbiotic gene regions: application to Rhizobium leguminosarum and its different biovars. Applied and Environmental Microbiology, 62: 2029-2036.

Laguerre G, Mazurier SI, Amarger N. 1992. Plasmid profiles and restriction fragment length polymorphism of Rhizobium leguminosarum bv. viciae in field populations. FEMS Microbiology Ecology, 10: 17-26.

Lakzian A, Murphy P, Turner A, Beynon JL, Giller KE. 2002. Rhizobium leguminosarum bv. viciae populations in soils with increasing heavy metal contamination: abundance, plasmid profiles, diversity and metal tolerance. Soil Biology \& Biochemistry, 34: 519529.

Langmead B, Salzberg SL. 2012. Fast gapped-read alignment with Bowtie 2. Nature Methods, 9: 357-U54.

Laslett D, Canback B. 2004. ARAGORN, a program to detect tRNA genes and tmRNA genes in nucleotide sequences. Nucleic Acids Research, 32: 11-16.

Laus MC, Logman TJ, Lamers GE, Van Brussel AAN, Carlson RW, Kijne JW. 2006. A novel polar surface polysaccharide from Rhizobium leguminosarum binds host plant lectin. Molecular Microbiology, 59: 1704-1713.

Leigh JA, Signer ER, Walker GC. 1985. Exopolysaccharide-deficient mutants of Rhizobium meliloti that form ineffective nodules. Proceedings of the National Academy of Sciences, 82: 6231-6235.

Leyva A, Palacios JM, Ruiz-Argüeso T. 1987. Conserved plasmid hydrogen-uptake (hup)specific sequences within Hup+ Rhizobium leguminosarum strains. Applied and Environmental Microbiology, 53: 2539-2543.

Li H, Handsaker B, Wysoker A, Fennell T, Ruan J, Homer N, Marth G, Abecasis G, Durbin R, Genome Project Data P. 2009. The sequence alignment/map format and SAMtools. Bioinformatics, 25: 2078-2079.

Liang Y, Cao Y, Tanaka K, Thibivilliers S, Wan J, Choi J, Kang Ch, Qiu J, Stacey G. 2013. Nonlegumes respond to rhizobial Nod Factors by suppressing the innate immune response. Science, 341: 1384-1387.

Libault M, Farmer A, Brechenmacher L, Drnevich J, Langley RJ, Bilgin DD, Radwan O, Neece DJ, Clough SJ, May GD, Stacey G. 2010. Complete transcriptome of the soybean root hair cell, a single-cell model, and its alteration in response to Bradyrhizobium japonicum nfection. Plant Physiology, 152: 541-552.

Limpens E, Zeijl Av, Geurts R. 2015. Lipochitooligosaccharides modulate plant host immunity to enable endosymbioses. Annual Review of Phytopathology, 53: 311-334.

Links MG, Demeke T, Gräfenhan T, Hill JE, Hemmingsen SM, Dumonceaux TJ. 2014. Simultaneous profiling of seed-associated bacteria and fungi reveals antagonistic interactions between microorganisms within a shared epiphytic microbiome on Triticum and Brassica seeds. New Phytologist, 202: 542-553.

Links MG, Dumonceaux TJ, Hemmingsen SM, Hill JE. 2012. The chaperonin-60 universal target is a barcode for bacteria that enables De Novo assembly of metagenomic sequence data. PLoS ONE, 7: e49755.

Lira MA, Nascimento LRS, Fracetto GGM. 2015. Legume-rhizobia signal exchange: promiscuity and environmental effects. Frontiers in Microbiology, 6: 945. 
Lodwig EM, Hosie AHF, Bourdes A, Findlay K, Allaway D, Karunakaran R, Downie JA, Poole PS. 2003. Amino-acid cycling drives nitrogen fixation in the legume-Rhizobium symbiosis. Nature, 422: 722-726.

Lohar DP, Sharopova N, Endre G, Peñuela S, Samac D, Town C, Silverstein KAT, VandenBosch KA. 2006. Transcript analysis of early nodulation events in Medicago truncatula. Plant Physiology, 140: 221-234.

Long SR. 1996. Rhizobium symbiosis: nod factors in perspective. Plant Cell, 8: 1885-98.

López M, Carbonero V, Cabrera E, Ruiz-Argüeso T. 1983. Effects of host on the expression of the H2-uptake hydrogenase of Rhizobium in legume nodules. Plant Science Letters, 29: 191-199.

Lopez NI, Floccari ME, Steinbuchel A, Garcia AF, Mendez BS. 1995. Effect of poly(3hydroxybutyrate) (PHB) content on the starvation-survival of bacteria in natural-waters. FEMS Microbiology Ecology, 16: 95-101.

Lopez-Gomez M, Sandal N, Stougaard J, Boller T. 2012. Interplay of flg22-induced defence responses and nodulation in Lotus japonicus. Journal of Experimental Botany, 63: 393401.

Loughney K, Lund E, Dahlberg JE. 1983. Deletion of an rRNA gene set in Bacillus subtilis. Journal of Bacteriology, 154: 529-532.

Louvrier P, Laguerre G, Amarger N. 1995. Semiselective medium for isolation of Rhizobium leguminosarum from soils. Soil Biology \& Biochemistry, 27: 919-924.

Louvrier P, Laguerre G, Amarger N. 1996. Distribution of symbiotic genotypes in Rhizobium leguminosarum biovar viciae populations isolated directly from soils. Applied and Environmental Microbiology, 62: 4202-4205.

Ludwig A, Jarchau T, Benz R, Goebel W. 1988. The repeat domain of Escherichia coli haemolysin (HlyA) is responsible for its $\mathrm{Ca2+-dependent} \mathrm{binding} \mathrm{to} \mathrm{erythrocytes.}$ Molecular and General Genetics MGG, 214: 553-561.

Lundberg DS, Lebeis SL, Paredes SH, Yourstone S, Gehring J, Malfatti S, Tremblay J, Engelbrektson A, Kunin V, del Rio TG, Edgar RC, Eickhorst T, Ley RE, Hugenholtz P, Tringe SG, Dangl JL. 2012. Defining the core Arabidopsis thaliana root microbiome. Nature, 488: 86-+.

Mandel MJ. 2010. Models and approaches to dissect host-symbiont specificity. Trends in Microbiology, 18: 504-511.

Marianelli C, Ciuchini F, Tarantino M, Pasquali P, Adone R. 2006. Molecular characterization of the rpoB gene in Brucella species: new potential molecular markers for genotyping. Microbes and Infection, 8: 860-865.

Martinez-Romero E. 2003. Diversity of Rhizobium-Phaseolus vulgaris symbiosis: overview and perspectives. Plant and Soil, 252: 11-23.

Mavingui P, Flores M, Guo X, Dávila G, Perret X, Broughton WJ, Palacios R. 2002. Dynamics of Genome Architecture in Rhizobium sp. Strain NGR234. Journal of Bacteriology, 184: 171-176.

Mclver J, Djordjevic MA, Weinman JJ, Bender GL, Rolfe BG. 1989. Extension of host range of Rhizobium leguminosarum bv. trifolii caused by point mutations in nodD that result in alterations in regulatory function and recognition of inducer molecules. Molecular PlantMicrobe Interactions, 2: 97-106.

Mergaert P, Uchiumi T, Alunni B, Evanno G, Cheron A, Catrice O, Mausset A-E, BarloyHubler F, Galibert F, Kondorosi A, Kondorosi E. 2006. Eukaryotic control on bacterial cell cycle and differentiation in the Rhizobium-legume symbiosis. Proceedings of the National Academy of Sciences, 103: 5230-5235.

Miranda-Sánchez F, Rivera J, Vinuesa P. 2015. Diversity patterns of Rhizobiaceae communities inhabiting soils, root surfaces and nodules reveal a strong selection of rhizobial partners by legumes. Environmental Microbiology: n/a-n/a.

Mitsui H, Gorlach K, Lee H-j, Hattori R, Hattori T. 1997. Incubation time and media requirements of culturable bacteria from different phylogenetic groups. Journal of Microbiological Methods, 30: 103-110.

Moawad H, Bohlool BB. 1984. Competition among Rhizobium spp. for nodulation of Leucaena leucocephala in two tropical soils. Applied and Environmental Microbiology, 48: 5-9.

Mollet C, Drancourt M, Raoult D. 1997. rpoB sequence analysis as a novel basis for bacterial identification. Molecular Microbiology, 26: 1005-1011. 
Morse R, Collins MD, Apos, Hanlon K, Wallbanks S, Richardson PT. 1996. Analysis of the $\beta$ 'Subunit of DNA-dependent RNA polymerase does not support the hypothesis inferred from 16S rRNA analysis that Oenococcus oeni (formerly Leuconostoc oenos) is a tachytelic (fast-evolving) bacterium. International Journal of Systematic and Evolutionary Microbiology, 46: 1004-1009.

Mortazavi A, Williams BA, McCue K, Schaeffer L, Wold B. 2008. Mapping and quantifying mammalian transcriptomes by RNA-Seq. Nature Methods, 5: 621-628.

Mutch LA, Tamimi SM, Young JPW. 2003. Genotypic characterisation of rhizobia nodulating Vicia faba from the soils of Jordan: a comparison with UK isolates. Soil Biology \& Biochemistry, 35: 709-714.

Mutch LA, Young JPW. 2004. Diversity and specificity of Rhizobium leguminosarum biovar viciae on wild and cultivated legumes. Molecular Ecology, 13: 2435-2444.

Nutman PS, Hearne R. 1979. Persistence of nodule bacteria in soil under long-term cereal cultivation. Rothamsted Experimental Station Report for 1969, part 2: 77-90.

Okonechnikov K, Conesa A, García-Alcalde F. 2015. Qualimap 2: advanced multi-sample quality control for high-throughput sequencing data. Bioinformatics.

Oldroyd GED. 2013. Speak, friend, and enter: signalling systems that promote beneficial symbiotic associations in plants. Nature Review Microbiology, 11: 252-263.

Oldroyd GED, Downie JA. 2008. Coordinating nodule morphogenesis with rhizobial infection in legumes. Annual Review of Plant Biology, 59: 519-546.

Oldroyd GED, Murray JD, Poole PS, Downie JA. 2011. The rules of engagement in the legume-rhizobial symbiosis. Annual Review of Genetics, 45: 119-144.

Oliver KL, Hamelin RC, Hintz WE. 2008. Effects of transgenic hybrid aspen overexpressing polyphenol oxidase on rhizosphere diversity. Applied and Environmental Microbiology, 74: 5340-5348.

Ottesen A, Gonzalez Pena A, White J, Pettengill J, Li C, Allard S, Rideout S, Allard M, Hill T, Evans P, Strain E, Musser S, Knight R, Brown E. 2013. Baseline survey of the anatomical microbial ecology of an important food plant: Solanum lycopersicum (tomato). BMC Microbiology, 13: 114.

Paau AS, Bloch CB, Brill WJ. 1980. Developmental fate of Rhizobium meliloti bacteroids in alfalfa nodules. Journal of Bacteriology, 143: 1480-1490.

Palmer KM, Young JPW. 2000. Higher diversity of Rhizobium leguminosarum biovar viciae populations in arable soils than in grass soils. Applied and Environmental Microbiology, 66: $2445-2450$.

Panday D, Schumann P, Das SK. 2011. Rhizobium pusense sp. nov., isolated from the rhizosphere of chickpea (Cicer arietinum L.). International Journal of Systematic and Evolutionary Microbiology, 61: 2632-2639.

Parente E, Guidone A, Matera A, De Filippis F, Mauriello G, Ricciardi A. 2016. Microbial community dynamics in thermophilic undefined milk starter cultures. International Journal of Food Microbiology, 217: 59-67.

Parniske M, Schmidt PE, Kosch K, Müller P. 1994. Plant defense responses of host plants with determinate nodules induced by EPS-defective exoB mutants of Bradyrhizobium japonicum. Molecular Plant-Microbe Interactions, 7: 631-638.

Patriarca EJ, Tatè R, laccarino M. 2002. Key role of bacterial $\mathrm{NH}(4)(+)$ metabolism in Rhizobium-plant symbiosis. Microbiology and Molecular Biology Reviews, 66: 203-222.

Pattison AC, Skinner FA. 1974. The effects of antimicrobial substances on Rhizobium spp. and their use in selective media. Journal of Applied Bacteriology, 37: 239-250.

Peck MC, Fisher RF, Long SR. 2006. Diverse flavonoids stimulate NodD1 binding to nod gene promoters in Sinorhizobium meliloti. Journal of Bacteriology, 188: 5417-5427.

Perret X, Staehelin C, Broughton WJ. 2000. Molecular basis of symbiotic promiscuity. Microbiology and Molecular Biology Reviews, 64: 180-201.

Popp C, Ott T. 2011. Regulation of signal transduction and bacterial infection during root nodule symbiosis. Current Opinion in Plant Biology, 14: 458-467.

Prell J, Poole P. 2006. Metabolic changes of rhizobia in legume nodules. Trends in Microbiology, 14: 161-168.

Price PA, Tanner HR, Dillon BA, Shabab M, Walker GC, Griffitts JS. 2015. Rhizobial peptidase HrrP cleaves host-encoded signaling peptides and mediates symbiotic compatibility. Proceedings of the National Academy of Sciences. 
Pühler G, Leffers H, Gropp F, Palm P, Klenk HP, Lottspeich F, Garrett RA, Zillig W. 1989. Archaebacterial DNA-dependent RNA polymerases testify to the evolution of the eukaryotic nuclear genome. Proceedings of the National Academy of Sciences, 86: 4569-4573.

Rådström P, Bäckman A, Qian N, Kragsbjerg P, Påhlson C, Olcén P. 1994. Detection of bacterial DNA in cerebrospinal fluid by an assay for simultaneous detection of Neisseria meningitidis, Haemophilus influenzae, and Streptococci using a seminested PCR strategy. Journal of Clinical Microbiology, 32: 2738-2744.

Rainey FA, Ward-Rainey NL, Janssen PH, Hippe H, Stackebrandt E. 1996. Clostridium paradoxum DSM 7308T contains multiple 16S rRNA genes with heterogeneous intervening sequences. Microbiology, 142: 2087-2095.

Rashid MH-o, Gonzalez J, Young JPW, Wink M. 2013. Rhizobium leguminosarum is the symbiont of lentils in the Middle East and Europe but not in Bangladesh. FEMS Microbiology Ecology: n/a-n/a.

Rashid MH-o, Young JPW, Everall I, Clercx P, Willems A, Santhosh Braun M, Wink M. 2015. Average nucleotide identity of genome sequences supports the description of Rhizobium lentis sp. nov., Rhizobium bangladeshense sp. nov. and Rhizobium binae sp. nov. from lentil (Lens culinaris) nodules. International Journal of Systematic and Evolutionary Microbiology, 65: 3037-3045.

Recourt K, van Brussel AA, Driessen AJ, Lugtenberg BJ. 1989. Accumulation of a nod gene inducer, the flavonoid naringenin, in the cytoplasmic membrane of Rhizobium leguminosarum biovar viciae is caused by the $\mathrm{pH}$-dependent hydrophobicity of naringenin. Journal of Bacteriology, 171: 4370-4377.

Reeve W, Ardley J, Tian R, Eshragi L, Yoon JW, Ngamwisetkun P, Seshadri R, Ivanova NN, Kyrpides NC. 2015. A Genomic Encyclopedia of the Root Nodule Bacteria: assessing genetic diversity through a systematic biogeographic survey. Standards in Genomic Sciences, 10: 14-14.

Reeve W, O'Hara G, Chain P, Ardley J, Brau L, Nandesena K, Tiwari R, Malfatti S, Kiss H, Lapidus A, Copeland A, Nolan M, Land M, Ivanova N, Mavromatis K, Markowitz V, Kyrpides N, Melino V, Denton M, Yates R, Howieson J. 2010a. Complete genome sequence of Rhizobium leguminosarum bv trifolii strain WSM2304, an effective microsymbiont of the South American clover Trifolium polymorphum. Standards in Genomic Sciences, 2: 66-76.

Reeve WG, \#039, hara G, Chain P, Ardley J, Bräu L, Nandesena K, Tiwari R, Copeland A, Nolan M, Han C, Brettin T, Land M, Ovchinnikova G, Ivanova N, Mavromatis K, Markowitz V, Kyrpides NC, Melino V, Denton M, Yates R, Howieson J. 2010b. Complete genome sequence of Rhizobium leguminosarum bv. trifolii strain WSM1325, an effective microsymbiont of annual Mediterranean clovers. Standards in Genomic Sciences, 2.

Riah N, Béna G, Djekoun A, Heulin K, de Lajudie P, Laguerre G. 2014. Genotypic and symbiotic diversity of Rhizobium populations associated with cultivated lentil and pea in sub-humid and semi-arid regions of Eastern Algeria. Systematic and Applied Microbiology.

Richter M, Rosselló-Móra R. 2009. Shifting the genomic gold standard for the prokaryotic species definition. Proceedings of the National Academy of Sciences, 106: 1912619131.

Rivilla R, Downie JA. 1994. Identification of a Rhizobium leguminosarum gene homologous to nodT but located outside the symbiotic plasmid. Gene, 144: 87-91.

Rivilla R, Sutton JM, Downie JA. 1995. Rhizobium leguminosarum NodT is related to a family of outer-membrane transport proteins that includes TolC, PrtF, CyaE and AprF. Gene, 161: $27-31$.

Robinson JT, Thorvaldsdottir H, Winckler W, Guttman M, Lander ES, Getz G, Mesirov JP. 2011. Integrative genomics viewer. Nature Biotechnology, 29: 24-26.

Roche P, Maillet F, Plazanet C, Debellé F, Ferro M, Truchet G, Promé J-C, Dénarié J. 1996. The common nodABC genes of Rhizobium meliloti are host-range determinants. Proceedings of the National Academy of Sciences, 93: 15305-15310.

Ruiz-Argüeso T, Hanus J, Evans HJ. 1983. Hydrogen production and uptake by pea nodules as affected by strains of Rhizobium leguminosarum. Archives of Microbiology, 116: 113118. 
Saito A, Mitsui H, Hattori R, Minamisawa K, Hattori T. 1998. Slow-growing and oligotrophic soil bacteria phylogenetically close to Bradyrhizobium japonicum. FEMS Microbiology Ecology, 25: 277-286.

Sambrook J, Russell DW. 2001. Molecular Cloning: A Laboratory Manual, Cold Spring Harbor Laboratory Press.

Sarita S, Sharma PK, Priefer UB, Prell J. 2005. Direct amplification of rhizobial nodC sequences from soil total DNA and comparison to nodC diversity of root nodule isolates. FEMS Microbiology Ecology, 54: 1-11.

Sawada H, Kuykendall LD, Young JM. 2003. Changing concepts in the systematics of bacterial nitrogen-fixing legume symbionts. The Journal of General and Applied Microbiology, 49: 155-179.

Schellenberg JJ, Links MG, Hill JE, Dumonceaux TJ, Kimani J, Jaoko W, Wachihi C, Mungai JN, Peters GA, Tyler S, Graham M, Severini A, Fowke KR, Ball TB, Plummer FA. 2011. Molecular definition of vaginal microbiota in East African commercial sex workers. Applied and Environmental Microbiology, 77: 4066-4074.

Schimel JP, Scott WJ, Killham K. 1989. Changes in cytoplasmic carbon and nitrogen pools in a soil bacterium and a fungus in response to salt stress. Applied and Environmental Microbiology, 55: 1635-1637.

Schlaeppi K, Dombrowski N, Oter RG, Ver Loren van Themaat E, Schulze-Lefert P. 2013. Quantitative divergence of the bacterial root microbiota in Arabidopsis thaliana relatives. Proceedings of the National Academy of Sciences.

Seemann T. 2014. Prokka: rapid prokaryotic genome annotation. Bioinformatics, 30: 20682069.

Segovia L, Pinero D, Palacios R, Martinezromero E. 1991. Genetic-structure of a soil population of nonsymbiotic Rhizobium leguminosarum. Applied and Environmental Microbiology, 57: 426-433.

Shaw LJ, Morris P, Hooker JE. 2006. Perception and modification of plant flavonoid signals by rhizosphere microorganisms. Environmental Microbiology, 8: 1867-1880.

Sherman VBD, McGowan V, Sneath PHA. 1980. Approved lists of bacterial names. International Journal of Systematic and Evolutionary Microbiology, 30: 225-420.

Shiraishi A, Matsushita N, Hougetsu T. 2010. Nodulation in black locust by the Gammaproteobacteria Pseudomonas sp. and the Betaproteobacteria Burkholderia sp. Systematic and Applied Microbiology, 33: 269-274.

Simsek S, Ojanen-Reuhs T, Stephens SB, Reuhs BL. 2007. Strain-ecotype specificity in Sinorhizobium meliloti-Medicago truncatula symbiosis is correlated to succinoglycan oligosaccharide structure. Journal of Bacteriology, 189: 7733-7740.

Smýkal P, Coyne CJ, Ambrose MJ, Maxted N, Schaefer H, Blair MW, Berger J, Greene SL, Nelson MN, Besharat N, Vymyslický T, Toker C, Saxena RK, Roorkiwal M, Pandey MK, Hu J, Li YH, Wang LX, Guo Y, Qiu LJ, Redden RJ, Varshney RK. 2015. Legume crops phylogeny and genetic diversity for science and breeding. Critical Reviews in Plant Sciences, 34: 43-104.

Sneath PHA, Sokal RR. 1973. Numerical taxonomy: the principles and practice of numerical classification, W. H. Freeman.

Soberón-Chávez G, Nájera R. 1989. Isolation from soil of Rhizobium leguminosarum lacking symbiotic information. Canadian Journal of Microbiology, 35: 464-468.

Somasegaran PHHJ. 1994. Handbook for Rhizobia : methods in legume-rhizobium technology, New York, Springer-Verlag.

Souza V, Eguiarte LE. 1997. Bacteria gone native vs. bacteria gone awry?: Plasmidic transfer and bacterial evolution. Proceedings of the National Academy of Sciences, 94: 55015503.

Spaepen S, Bossuyt S, Engelen K, Marchal K, Vanderleyden J. 2014. Phenotypical and molecular responses of Arabidopsis thaliana roots as a result of inoculation with the auxin-producing bacterium Azospirillum brasilense. New Phytologist, 201: 850-861.

Sprent JI, Minchin FR, Parsons R. 1993. Evolution since Knoxville: were nitrogen-fixing organisms wise to inhabit land plants? In: Palacios R, Mora J, Newton WE eds. New Horizons in Nitrogen Fixation: Proceedings of the 9th International Congress on Nitrogen Fixation, Cancún, Mexico, December 6-12, 1992. Dordrecht, Springer Netherlands. 
Sprent JI, Sutherland JM, de Faria SM, Dilworth MJ, Corby HDL, Becking JH, Materon LA, Drozd JW. 1987. Some aspects of the biology of nitrogen-fixing oganisms Philosophical Transactions of the Royal Society of London B: Biological Sciences, 317: 111-129.

Strain SR, Leung K, Whittam TS, de Bruijn FJ, Bottomley PJ. 1994. Genetic structure of Rhizobium leguminosarum biovar trifolii and viciae populations found in two Oregon soils under different plant communities. Applied and Environmental Microbiology, 60: 2772-2778.

Streeter JG. 1994. Failure of inoculant rhizobia to overcome the dominance of indigenous strains for nodule formation. Canadian Journal of Microbiology, 40: 513-522.

Streit W, Kipe-Nolt J, Werner D. 1991. Competitive growth of Rhizobium leguminosarum bv. phaseoli strains under oligotrophic conditions. Current Microbiology, 23: 159-163.

Sugawara M, Epstein B, Badgley BD, Unno T, Xu L, Reese J, Gyaneshwar P, Denny R, Mudge J, Bharti AK, Farmer AD, May GD, Woodward JE, Médigue C, Vallenet D, Lajus A, Rouy Z, Martinez-Vaz B, Tiffin P, Young ND, Sadowsky MJ. 2013. Comparative genomics of the core and accessory genomes of 48 Sinorhizobium strains comprising five genospecies. Genome Biology, 14: 1-20.

Sullivan JT, Eardly BD, VanBerkum P, Ronson CW. 1996. Four unnamed species of nonsymbiotic rhizobia isolated from the rhizosphere of Lotus corniculatus. Applied and Environmental Microbiology, 62: 2818-2825.

Sullivan JT, Patrick HN, Lowther WL, Scott DB, Ronson CW. 1995. Nodulating strains of Rhizobium loti arise through chromosomal symbiotic gene transfer in the environment. Proceedings of the National Academy of Sciences, 92: 8985-8989.

Surin BP, Downie JA. 1989. Rhizobium leguminosarum genes required for expression and transfer of host specific nodulation. Plant Molecular Biology, 12: 19-29.

Surin BP, Watson JM, Hamilton WDO, Economou A, Downie JA. 1990. Molecular characterization of the nodulation gene, nodT, from two biovars of Rhizobium leguminosarum. Molecular Microbiology, 4: 245-252.

Sutton JM, Lea EJ, Downie JA. 1994. The nodulation-signaling protein NodO from Rhizobium leguminosarum biovar viciae forms ion channels in membranes. Proceedings of the National Academy of Sciences, 91: 9990-9994.

Sutton JM, Peart J, Dean G, Downie JA. 1996. Analysis of the C-terminal secretion signal of the Rhizobium leguminosarum nodulation protein NodO; a potential system for the secretion of heterologous proteins during nodule invasion. Molecular Plant-Microbe Interactions, 9: 671-80.

Swain MT, Tsai IJ, Assefa SA, Newbold C, Berriman M, Otto TD. 2012. A Post-assembly genome-improvement toolkit (PAGIT) to obtain annotated genomes from contigs. Nature protocols, 7: 1260-1284.

Tamura K, Stecher G, Peterson D, Filipski A, Kumar S. 2013. MEGA6: Molecular Evolutionary Genetics Analysis Version 6.0. Molecular Biology and Evolution, 30: 27252729.

Tilman D, Cassman KG, Matson PA, Naylor R, Polasky S. 2002. Agricultural sustainability and intensive production practices. Nature, 418: 671-677.

Timmers ACJ, Soupene E, Auriac MC, de Billy F, Vasse J, Boistard P, Truchet G. 2000. Saprophytic intracellular rhizobia in alfalfa nodules. Molecular Plant-Microbe Interactions, 13: 1204-1213.

Tittabutr P, Piromyou P, Longtonglang A, Noisa-Ngiam R, Boonkerd N, Teaumroong $\mathbf{N}$. 2013. Alleviation of the effect of environmental stresses using co-inoculation of mungbean by Bradyrhizobium and rhizobacteria containing stress-induced ACC deaminase enzyme. Soil Science and Plant Nutrition, 59: 559-571.

Tkacz A, Cheema J, Chandra G, Grant A, Poole PS. 2015. Stability and succession of the rhizosphere microbiota depends upon plant type and soil composition. ISME Journal.

Tong Z, Sadowsky MJ. 1994. A selective medium for the isolation and quantification of Bradyrhizobium japonicum and Bradyrhizobium elkanii strains from soils and inoculants. Applied and Environmental Microbiology, 60: 581-586.

Triplett EW, Albrecht KA, Oplinger ES. 1993. Crop rotation effects on populations of Bradyrhizobium japonicum and Rhizobium meliloti. Soil Biology \& Biochemistry, 25: 781-784.

Tsai I, Otto T, Berriman M. 2010. Improving draft assemblies by iterative mapping and assembly of short reads to eliminate gaps. Genome Biology, 11: R41. 
Tyler HL, Roesch LFW, Gowda S, Dawson WO, Triplett EW. 2009. Confirmation of the sequence of 'Candidatus Liberibacter asiaticus' and assessment of microbial diversity in Huanglongbing-infected citrus phloem using a metagenomic approach. Molecular PlantMicrobe Interactions, 22: 1624-1634.

Vance CP, Johnson LEB, Halvorsen AM, Heichel GH, Barnes DK. 1980. Histological and ultrastructural observations of Medicago sativa root nodule senescence after foliage removal. Canadian Journal of Botany, 58: 295-309.

Vanderlinde EM, Harrison JJ, Muszyński A, Carlson RW, Turner RJ, Yost CK. 2010. Identification of a novel $A B C$ transporter required for desiccation tolerance, and biofilm formation in Rhizobium leguminosarum bv. viciae 3841. FEMS Microbiology Ecology, 71: 327-340.

Vanrhijn P, Vanderleyden J. 1995. The Rhizobium-plant symbiosis. Microbiological Reviews, 59: 124-142.

Vasse J, Debilly F, Camut S, Truchet G. 1990. Correlation between ultrastructural differentiation of bacteroids and nitrogen-fixation in alfalfa nodules. Journal of Bacteriology, 172: 4295-4306.

Vesth T, Lagesen K, Acar n, Ussery D. 2013. CMG-Biotools, a free workbench for basic comparative microbial genomics. PLoS ONE, 8: e60120.

Větrovský T, Baldrian P. 2013. The variability of the $16 \mathrm{~S}$ rRNA gene in bacterial genomes and its consequences for bacterial community analyses. PLoS ONE, 8: e57923.

Vincent JM. 1970. A manual for the practical study of root-nodule bacteria, Oxford, International Biological Programme, Blackwell Scientific.

Vos M, Quince C, Pijl AS, de Hollander M, Kowalchuk GA. 2012. A comparison of rpoB and $16 \mathrm{~S}$ rRNA as markers in pyrosequencing studies of bacterial diversity. PLOS ONE, 7.

Walker SA, Downie JA. 2000. Entry of Rhizobium leguminosarum bv. viciae into root hairs requires minimal Nod Factor specificity, but subsequent infection thread growth requires nodO or nodE. Molecular Plant-Microbe Interactions, 13: 754-762.

Wang D, Yang S, Tang F, Zhu H. 2012. Symbiosis specificity in the legume - rhizobial mutualism. Cellular Microbiology, 14: 334-342.

Wassem R, Kobayashi H, Kambara K, Le Quéré A, Walker GC, Broughton WJ, Deakin WJ. 2008. Ttsl regulates symbiotic genes in Rhizobium species NGR234 by binding to tts boxes. Molecular Microbiology, 68: 736-748.

Werner GDA, Cornwell WK, Sprent JI, Kattge J, Kiers ET. 2014. A single evolutionary innovation drives the deep evolution of symbiotic N2-fixation in angiosperms. Nature Communications, 5.

White PJ, Brown PH. 2010. Plant nutrition for sustainable development and global health. Annals of Botany, 105: 1073-1080.

Wilson K. 2001. Preparation of genomic DNA from bacteria. Current protocols in molecular biology / edited by Frederick M. Ausubel ... [et al.], Chapter 2: Unit 2.4-Unit 2.4.

Woese CR. 1987. Bacterial evolution. Microbiological Reviews, 51: 221-271.

Woese CR, Kandler O, Wheelis ML. 1990. Towards a natural system of organisms - proposal for the domains Archaea, Bacteria, and Eukarya. Proceedings of the National Academy of Sciences, 87: 4576-4579.

Wright S. 1978. Evolution and the genetics of populations, volume 4: Variability within and among natural populations, University of Chicago Press.

Yang L, Duff M, Graveley B, Carmichael G, Chen L-L. 2011. Genomewide characterization of non-polyadenylated RNAs. Genome Biology, 12: R16.

Yang S, Tang F, Gao M, Krishnan HB, Zhu H. 2010. R gene-controlled host specificity in the legume-rhizobia symbiosis. Proceedings of the National Academy of Sciences, 107: $18735-18740$.

Yeoh YK, Paungfoo-Lonhienne C, Dennis PG, Robinson N, Ragan MA, Schmidt S, Hugenholtz P. 2015. The core root microbiome of sugarcanes cultivated under varying nitrogen fertiliser application. Environmental Microbiology: n/a-n/a.

Yoshida K-i, Takemoto Y, Sotsuka T, Tanaka K, Takenaka S. 2013. PhaP phasins play a principal role in poly- $\beta$-hydroxybutyrate accumulation in free-living Bradyrhizobium japonicum. BMC Microbiology, 13: 1-10.

Young J. 1992. Phylogenetic classification of nitrogen-fixing organisms. In: Stace G, Burris R, Evans $\mathrm{H}$ eds. Biological Nitrogen Fixation. New York, Chapman \& Hall. 
Young JM, Kuykendall LD, Martínez-Romero E, Kerr A, Sawada H. 2001. A revision of Rhizobium Frank 1889, with an emended description of the genus, and the inclusion of all species of Agrobacterium Conn 1942 and Allorhizobium undicola de Lajudie et al. 1998 as new combinations: Rhizobium radiobacter, $R$. rhizogenes, $R$. rubi, $R$. undicola and $R$. vitis. International Journal of Systematic and Evolutionary Microbiology, 51: 89103.

Young JPW, Crossman LC, Johnston AWB, Thomson NR, Ghazoui ZF, Hull KH, Wexler M, Curson ARJ, Todd JD, Poole PS, Mauchline TH, East AK, Quail MA, Churcher C, Arrowsmith C, Cherevach I, Chillingworth T, Clarke K, Cronin A, Davis P, Fraser A, Hance Z, Hauser H, Jagels K, Moule S, Mungall K, Norbertczak H, Rabbinowitsch E, Sanders M, Simmonds M, Whitehead S, Parkhill J. 2006. The genome of Rhizobium leguminosarum has recognizable core and accessory components. Genome Biology.

Zeze A, Mutch LA, Young JPW. 2001. Direct amplification of nodD from community DNA reveals the genetic diversity of Rhizobium leguminosarum in soil. Environmental Microbiology, 3: 363-370.

Zhang X, Sun L, Ma X, Sui XH, Jiang R. 2011. Rhizobium pseudoryzae sp. nov., isolated from the rhizosphere of rice. International Journal of Systematic and Evolutionary Microbiology, 61: 2425-2429.

Zhang XX, Kosier B, Priefer UB. 2001. Genetic diversity of indigenous Rhizobium leguminosarum bv. viciae isolates nodulating two different host plants during soil restoration with alfalfa. Molecular Ecology, 10: 2297-2305. 


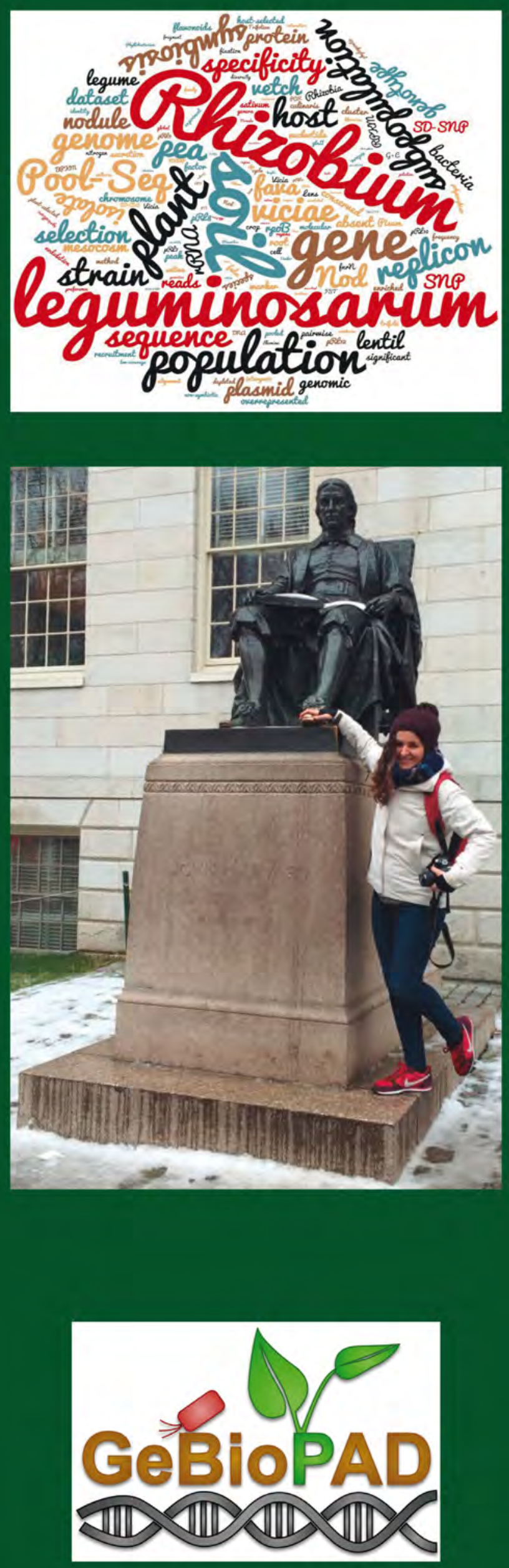

Genómica y Biotecnología de Bacterias Diazotróficas Asociadas con Plantas Centro de Biotecnología y Genómica de Plantas (UPM-INIA)

IP: Juan Imperial 